\title{
A NOVEL UNIT CELL ANTENNA FOR HIGHLY INTEGRATED \\ PHASED ARRAYS IN THE SHF BAND
}

\author{
A Thesis \\ presented to \\ the Faculty of California Polytechnic State University, \\ San Luis Obispo
}

\author{
In Partial Fulfillment \\ of the Requirements for the Degree \\ Master of Science in Electrical Engineering
}

by

Timothy Bryan Ogilvie

June 2013 
(C) 2013

Timothy Bryan Ogilvie

ALL RIGHTS RESERVED 


\section{COMMITTEE MEMBERSHIP}

TITLE:

A NOVEL UNIT CELL ANTENNA FOR HIGHLY INTEGRATED PHASED ARRAYS IN THE SHF BAND

AUTHOR:

DATE SUBMITTED:

COMMITTEE CHAIR:

COMMITTEE MEMBER:

COMMITTEE MEMBER:
Timothy Bryan Ogilvie

June 2013
Dr. Dennis Derickson, Electrical Engineering Department Chair

Dr. Xiaomin Jin, Associate Professor of Electrical Engineering

Dr. Vladimir Prodanov, Assistant Professor of Electrical Engineering 


\section{ABSTRACT \\ A NOVEL UNIT CELL ANTENNA FOR HIGHLY INTEGRATED \\ PHASED ARRAYS IN THE SHF BAND}

Timothy B. Ogilvie

Phased arrays are electromagnetic antenna systems comprised of many radiating elements and processing electronics. Radiating elements are typically positioned in an orderly grid within the antenna aperture. In the receive mode of operation, radiating elements capture some of the signal energy from incoming radiation and guide these signals to processing electronics. Signals are filtered and amplified to maintain the desired sensitivity and complexly weighted using circuits with reconfigurable amplification gain and phase delay. Finally, all signals are combined. The summation of these complexly weighted spatial samples forms a spatial filter in the same way complexly weighted temporal samples establish a temporal filter in a finite impulse response discrete-time filter. Therefore, a phased array behaves like a spatial filter that strongly favors signals arriving from a specific direction. This favored direction represents the look angle of its beam, and the shape of the beam directly relates to the complex weights applied to the signals in the array. Analogous to the flexibility offered by digital filters, phased arrays enable agile beam steering, sidelobe control, and multiple independent beams. These capabilities have revolutionized radar, radioastronomy, and communication systems.

Phased arrays have increasingly employed printed circuit board (PCB) fabrication techniques and processes to maximize array channel density, achieve lower profile, and minimize component integration cost. A few applications which leverage these qualities include low-cost radar, mobile satellite communication (SATCOM), and intelligence, surveillance, and reconnaissance (ISR). Further, PCB-based arrays readily accommodate advancements in highly integrated beamforming radio frequency integrated circuits (RFICs), multi-chip modules, and RF micro-electromechanical system (MEMS) device technologies.

On a prior effort, an integrated unit cell design was developed for a PCB-based SATCOM array application. However, the design failed to meet the requirements. The primary objective of this work is to demonstrate an improved design using systematic microwave design techniques and modern analysis tools to meet the requirements for the same application. The proposed design must improve gain, bandwidth, size, and manufacturability over the prior design. Additionally, the design must be generally extensible to phased array implementations across the SHF band (3-30 GHz).

This work discusses the advantages of phased arrays over continuous apertures (e.g. reflectors), reviews phased array theory, and proposes an improved unit cell design. The proposed design is $35 \%$ smaller than a dime and consists of an orthogonally-fed, slot-coupled stacked patch antenna and dual-stage branchline coupler implemented in a multilayer PCB. Within the operating band from 10.7 to $14.5 \mathrm{GHz}$, the design achieves an average return loss of $15 \mathrm{~dB}$, a uniform radiation pattern with peak realized gain of 4.8 to $7.0 \mathrm{dBic}$, cross-polarization level below $-17 \mathrm{~dB}$, and stable performance in a closely-spaced array. When configured in an array, the design supports $\mathrm{X} / \mathrm{Ku}$-band SATCOM in full-duplex operation, electronically rotatable polarization, and a $47.5^{\circ}$ grating lobe free conical scan range. Further, a Monte Carlo analysis proves the design accommodates tolerances of material properties and manufacturing processes, overcoming a major challenge in PCB-based high frequency antenna design. 


\section{ACKNOWLEDGMENTS}

It is a privilege to acknowledge a few sources of inspiration largely responsible for my education and the general direction of my life: family, friends, teachers, and colleagues.

I inherit from my father, among other things, a curiosity and passion to search for truth, the diligence to guide me closer, and the impetus to share the things I learn along the way. He is a genuinely good man and loving father, and he demonstrates the merits of balancing work affairs and family life - something he does remarkably well. These attributes will continue to guide and influence my personal and professional development.

My mother's undying love is a primary source of strength and contentment, an infinitely deep well from which I draw courage to keep learning, keep loving, and keep pushing - for the way to eat an elephant is one small bite at a time. She is truly a remarkable individual and exceptional mother; I'm indebted to the stork that left me in her care.

My stepfather affords me the opportunity to study in San Luis Obispo and continues to encourage, love, and support me and my endeavors. Among others, he has imparted in me an appreciation for music and the courage to walk to the beat of my own drum. Both are treasured elements in my life.

My stepmother tirelessly devotes her entire being to the care of my family. She has done a remarkable job mothering my brothers and sisters: Austin, Kristiana, Jonathan, Nickolas, and Mariana. Above all, she imparts in me an appreciation for the difficult and delicate job of raising children. The fruits of her labor will continue to blossom for years to come.

To my love Rebecca - you have been incredibly supportive and loving when I'm not so lovable. I appreciate your honesty, kindness, and all the hard work you put in to make our little family function. I sincerely look forward to many more years with you and the pups.

To my friends, I owe a special thanks for putting up with all the nights and weekends I forfeited in my quest to progress on this work. It has been a long journey.

I extend gratitude to a few inspirational teachers: Ron Brown, Jerome Breitenbach, and Jim Harris. I sincerely appreciate the memorable lectures, handcrafted assignments, and valuable advice. A sincere thanks also extends to Dennis Derickson and Yvonne Lynch for the coordination and positive encouragement leading to the completion of this work.

Lastly, to my colleagues at Space Systems Company, with whom I am afforded the opportunity to bridge the gap between concepts and practice. For me, this process is exceptionally rewarding. I extend a special appreciation for the mentorship of Larry Lam and Anthony JacombHood. From them, I have learned most of what I know about phased array systems. It is an honor to work alongside them on the most advanced phased array systems in the world.

I conclude with a remark and challenge to readers. In this work, I present a unit cell antenna design for an application that might allow internet or television access to passengers aboard mobile aircraft, cruise ships, or personal vehicles. I selected this application because it is widely recognizable and extends beyond conventional military radar and intelligence systems. It is no secret that war has been and continues to be the primary catalyst for investment and advancement of phased array technologies. My fascination with phased arrays developed while working on a design challenge for an antennas course when the simple thought occurred to me: very profound things are possible when a collection of imperfect elements are working cooperatively. I believe this is a fundamental message for us all. It is my sincere hope that we can divest from the status quo and innovatively repurpose phased arrays for the enrichment and improvement of humanity. 


\section{TABLE OF CONTENTS}

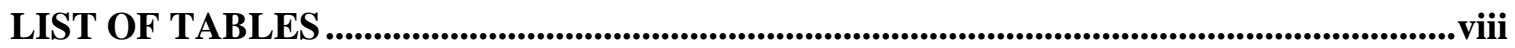

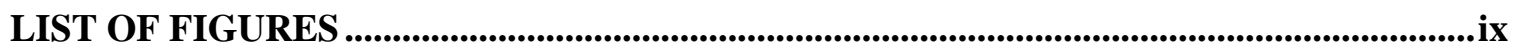

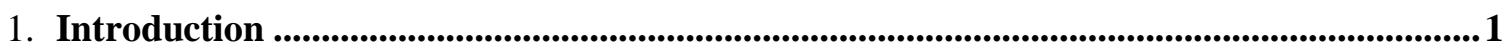

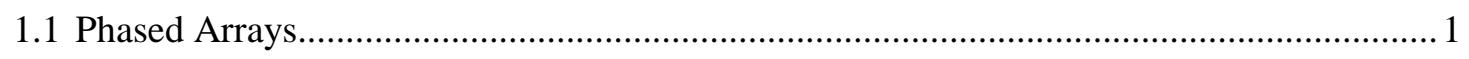

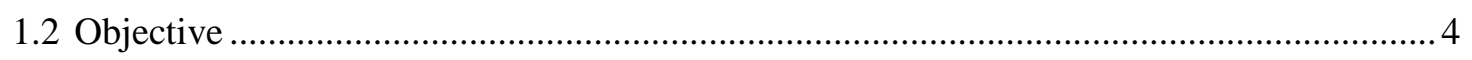

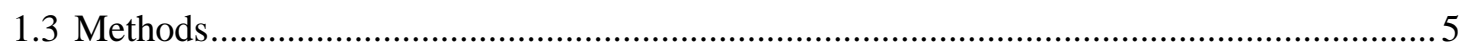

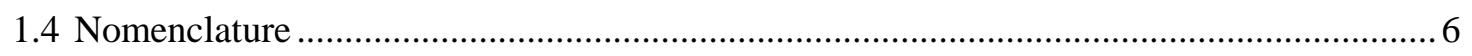

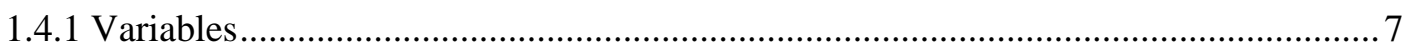



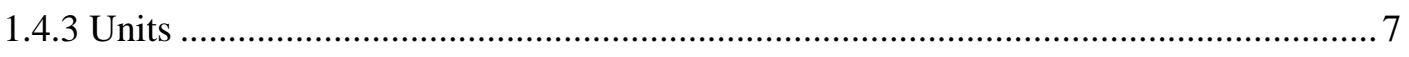

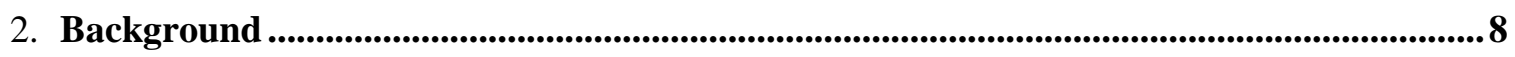

2.1 Historical Achievements Preceding the Reflector Antenna ............................................ 8

2.2 The Significance of the Reflector Antenna ................................................................... 16

2.3 The Advantages of the Phased Array Antenna ............................................................. 19

3. Phased Array Theory .............................................................................................................................26

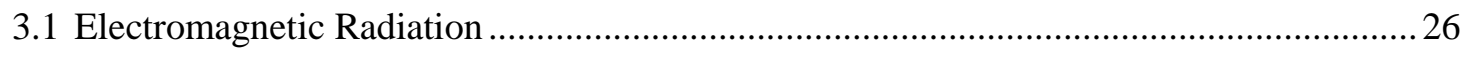

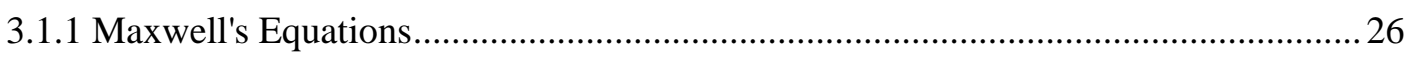

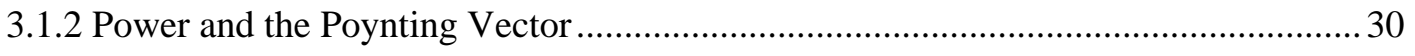

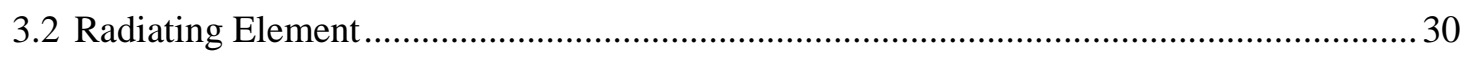

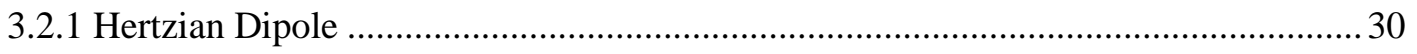

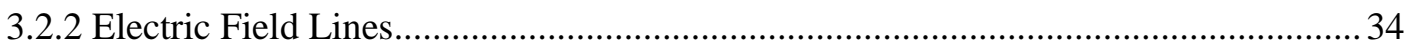

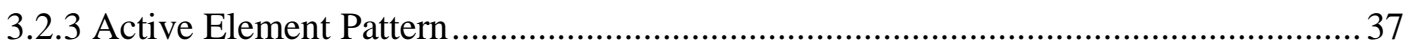

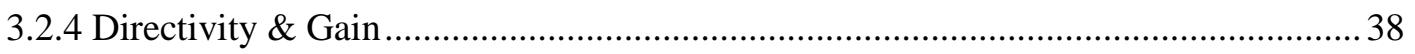

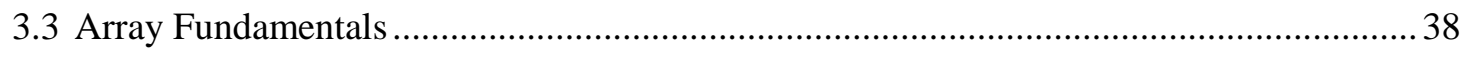

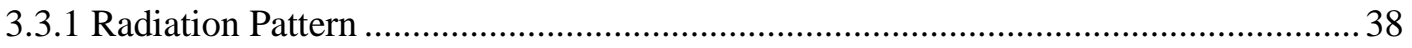

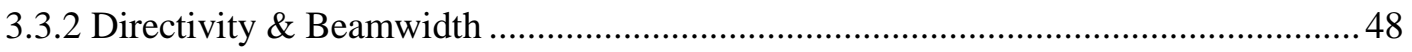

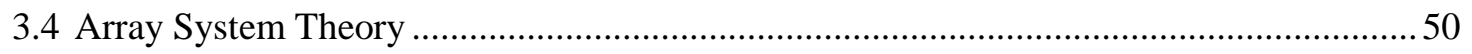

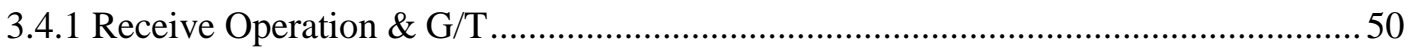

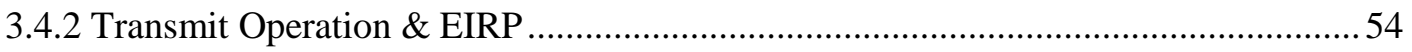

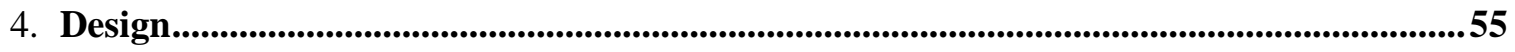

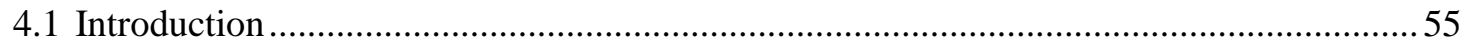

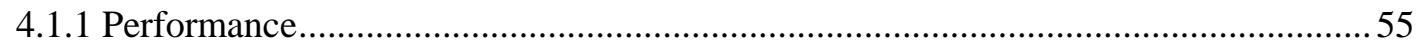




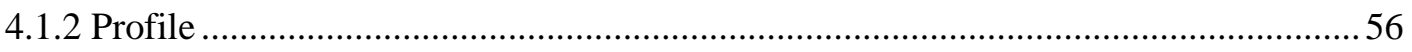

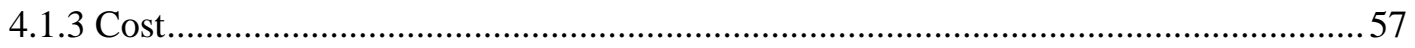

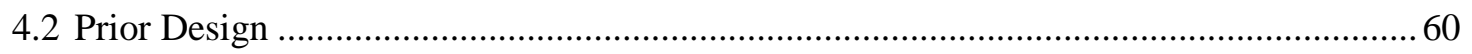

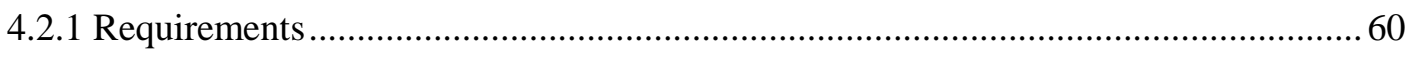

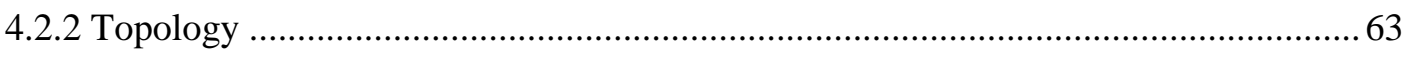

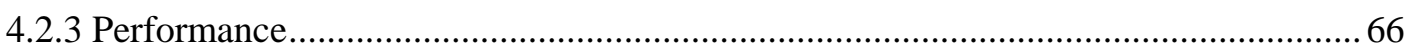

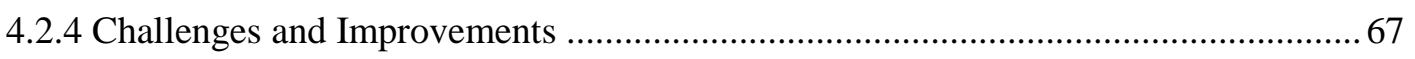

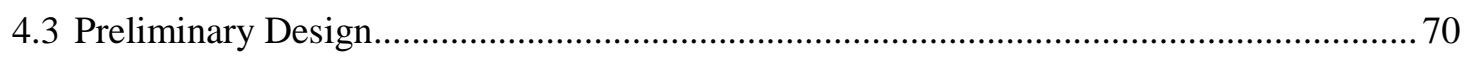

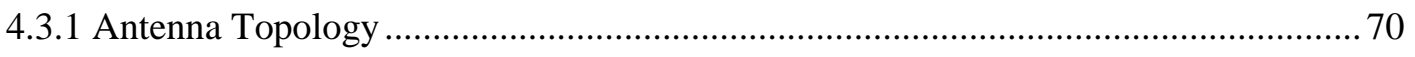

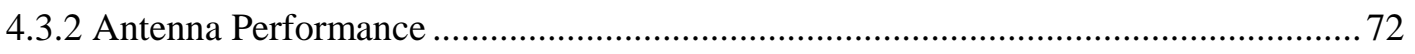

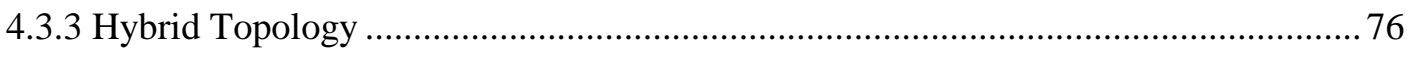

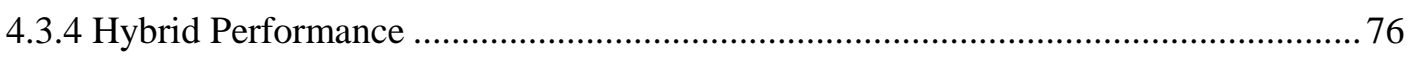

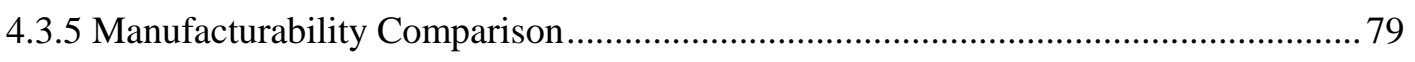

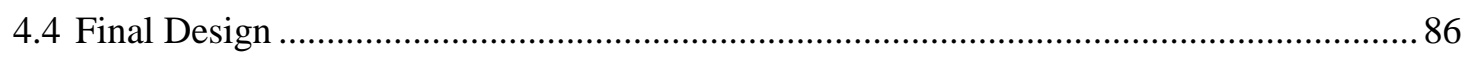

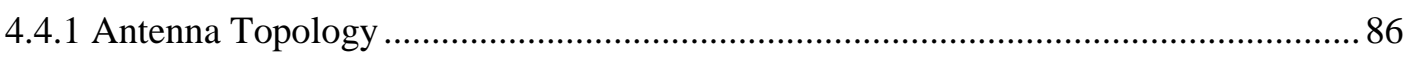

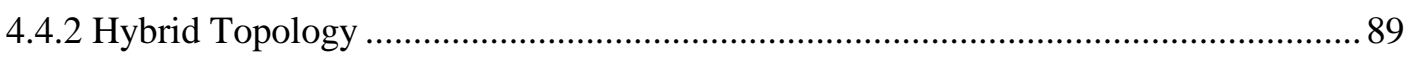

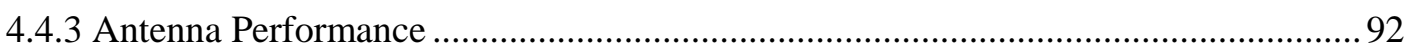

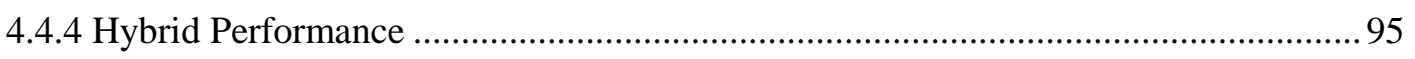

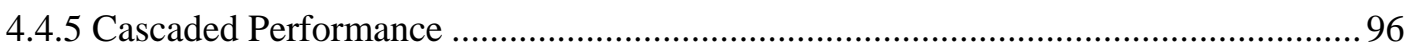

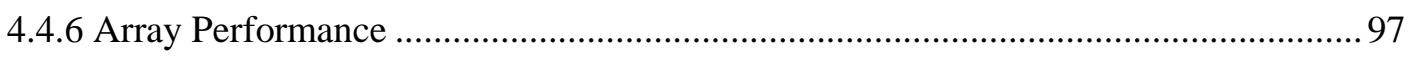

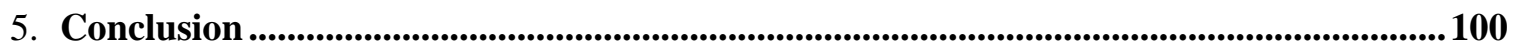

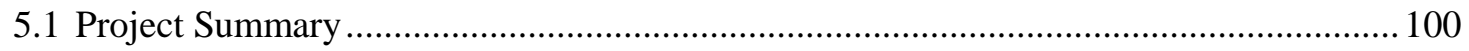

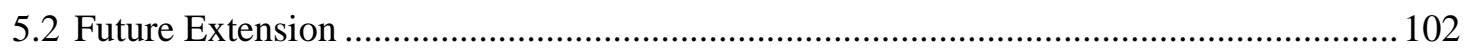



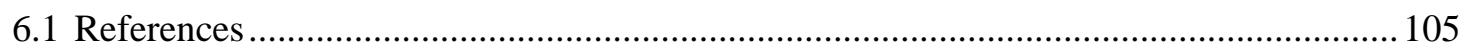

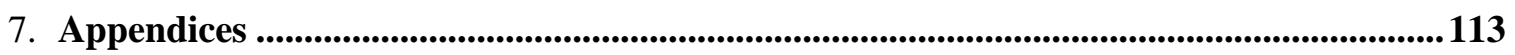

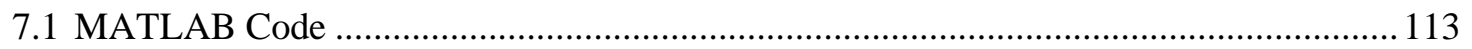

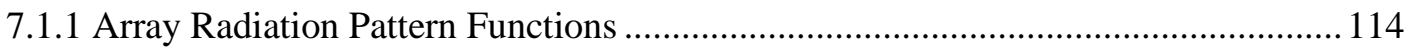

7.1.2 Element Radiation Pattern Functions ...................................................................... 114

7.1.3 Hertzian Dipole Visualization Functions ............................................................... 117

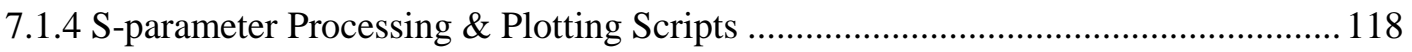




\section{LIST OF TABLES}

Table 3.3.1 Directivity-beamwidth products for common aperture distributions.

Table 4.1.1 Bill of material cost for an X/Ku-band full-duplex satellite communications array illustrates marginalization of unit cell radiating element cost afforded by PCB implementation for three build quantities. This table also highlights the nontrivial cost and unfavorable cost scaling over quantity for connectors and cables.

Table 4.2.1 Requirements for the dual-circularly polarized X/Ku-band unit cell antenna and hybrid. Realized antenna gain expressed in units of $\mathrm{dBic}$ represents gain for circular polarization assuming the dual-linearly polarized antenna is fed with an ideal (lossless) $90^{\circ}$ hybrid coupler.

Table 4.2.2 Prior unit cell antenna and hybrid simulated performance compared to the requirements. Nominal values for material properties and model dimensions are assumed.

Table 4.3.1 Performance comparison of the preliminary and prior antenna and hybrid designs compared to the requirements. Nominal values for material properties and model dimensions are assumed.

Table 4.3.2 Material properties, dimensions, tolerances, and descriptions for all materials included in the Monte Carlo analysis for the preliminary antenna design. Tolerances are derived from Rogers Corporation's High Frequency Materials Product Selection Guide [81]. Model parameters are defined in Figure 4.3.2 and Figure 4.3.3.

Table 4.3.3 Manufacturing process tolerances are applied to model dimensions in the Monte Carlo analysis for the preliminary antenna design. All tolerances are derived from process capabilities of modern PCB manufacturers.

Table 4.4.1 Performance comparison of final, preliminary, and prior antenna and hybrid designs compared with the requirements for each design. Nominal values for material properties and model dimensions are assumed. 


\section{LIST OF FIGURES}

Figure 1.1.1 A general concept of a phased array antenna illustrates radiation from source current distributions $J i$ oriented in space and phased in time such that radiated fields constructively interfere in the desired direction of point $\mathrm{P}$. ............................. 1

Figure 1.1.2 Planar phased arrays implemented as multilayer PCBs are comprised of many unit cell antennas, combiners, and solder attached beamforming electronics. One primary challenge is to design an electromagnetically robust structure that yields a well-behaved radiation pattern in a dynamic environment and can withstand variation associated with PCB materials and manufacturing process tolerances. 3

Figure 1.1.3 A generalized analog full-duplex phased array front end consists of antenna elements, band or beam switches, filters, variable amplifiers, phase shifters or time delay units, and coherent summers. This paper describes the flexibility offered by such a front end and focuses on the antenna element design. 4

Figure 2.1.1 Parabolic curve represented as a conic section illustrating the concept of the focus in the context of Euclidean geometry.

Figure 2.1.2 Parabolic curve illustrating the concept of the focus in the context of Cartesian geometry.

Figure 2.1.3 Reflective telescope designs popularized by (a) Isaac Newton, (b) James Gregory, and (c) Laurent Cassegrain [5]

Figure 2.1.4 The James Webb Space Telescope (JWST) satellite system depicting its Cassegrain three-mirror anastigmatic optical system and thermal shields [6]. Images courtesy of NASA.

Figure 2.1.5 The 455-MHz cylindrical parabolic reflector antenna fed by a dipole antenna and spark-gap transmitter invented by Heinrich Hertz in $1888[16,17]$.

Figure 2.2.1 The Karl G. Jansky Very Large Array (VLA) radio telescope utilizes 27 different parabolic reflectors that are each 25 meters in diameter to observe galaxies and other astronomical objects. Both photographs were taken by Dave Finley. Image courtesy of NRAO/AUI website at http://www.nrao.edu.

Figure 2.2.2 Colorful satellite dish antennas adorn landscapes in cities around the world. This photograph was taken by Carlos Jasso of Reuters in Mexico City on July 
12, 2011. It reflects the prevalence of media access in even the poorest of developing nations. The reflector antenna serves as a cost-effective solution for this application.

Figure 2.2.3 The Mobile User Objective System (MUOS) is the next generation narrowband communications system for the United States. (a) Each satellite relies on two deployable gold mesh reflectors which are 5.4 and 14 meters in diameter, and (b) each earth terminal in relies on a reflector antenna that is roughly 20 meters in diameter [26].

Figure 2.3.1 Qualitative depiction of the similarities between (a) the parabolic reflector antenna and (c) the phased array antenna. A parabolic reflector antenna is represented as a continuous aperture relying on a spatial summing node. A phased array is represented as a sampled aperture where samples are complexly weighted and summed using beamforming electronics.

Figure 2.3.2 Three primary advantages of the phased array antenna: (a) sidelobe control, (b) beam scanning, and (c) arbitrary multi-beam shaping. .25

Figure 3.1.1 Geometry employed to compute the magnetic vector potential $A$ and corresponding electric and magnetic fields $E$ and $H$ at a given point $\mathrm{P}$ resulting from a source current distribution $J$ impressed upon source volume $v^{\prime}$ evaluated at distance vectors $r=r p-r s$.

Figure 3.2.1 Geometry employed to compute the magnetic vector potential $A$ and corresponding electric and magnetic fields $E$ and $H$ at a given point $\mathrm{P}$ resulting from a source current distribution $J$ associated with a y-directed current source of infinitesimal length.

Figure 3.2.2 The electric field lines of a Hertzian dipole on the $x z$-plane at time $t 0+n T / 8$ where $T=2 \pi / \omega$ and (a) $n=0$, (b) $n=1$, (c) $n=2$, and (d) $n=3$. The left figures are contour plots generated in MATLAB, and the right figures are screenshots from Hertz's historical paper in 1893 [17]. .37

Figure 3.3.1 A one dimensional array described by lattice parameter $d$. .39

Figure 3.3.2 Coordinate geometry to facilitate the construction of assumptions employed to simplify the expression for array field intensity. 
Figure 3.3.3 (a) A general lattice of a two dimensional array is uniquely described by lattice parameters $[d, b, \gamma]$ and related to the location of each element $(x n, y n)$ by (3.59). Two common configurations are (b) the rectangular lattice described by $\left[d x, b y, 90^{\circ}\right]$ and (c) the equilateral triangular lattice described by $\left[d x, 0.866 d x, 60^{\circ}\right]$.

Figure 3.3.4 A 16 × 16 element array oriented in (a) an equilateral triangular lattice produces the $3 \mathrm{D}$ and $2 \mathrm{D}$ radiation patterns for $a n=1 \forall n$ in (b) and (c), respectively, computed at $14.5 \mathrm{GHz}$.

Figure 3.4.1 Cascaded receive chain used to compute system noise temperature and G/T of a PCB-based X/Ku-band SATCOM array. The sky temperature Tsky is a dynamic parameter that depends on many variables, including weather and other atmospheric conditions. For this application, atmospheric gases and weather-dependent phenomena primarily contribute to sky temperature [60, 61]. Each element in the chain is represented by a gain and noise figure in Figure 3.4.2. The hybrid converts dual-linear to dual-circular polarization. The duplexer sets the noise bandwidth of the system and enables full-duplex operation since both Rx and Tx chains share the same antenna. The LNA is responsible for marginalizing downstream loss impacts to the system noise temperature. An RFIC is assumed to implement beamforming functionality (amplitude and phase offsets) as well as integrate combiners, memory, and a serial communication interface. The final block represents the losses encountered in the subsequent combiners and the down-conversion stage. Board loss elements represent transmission line and transition losses.

Figure 3.4.2 Cascaded system noise temperature calculated at 10.7, 11.7, and 12.7 GHz for a $\mathrm{X} / \mathrm{Ku}$-band SATCOM array. The primary input variables to the table are highlighted yellow, and the computed results are highlighted blue. A sky noise temperature of $15 \mathrm{~K}$ assumes moderate humidity and heavy cloud conditions. Moderate humidity is defined as water vapor density of $5-20 \mathrm{~g} / \mathrm{m}^{3}$, and a heavy cloud condition is defined as liquid water content of $0.5 \mathrm{~g} / \mathrm{m}^{3}$ in the Slobin cloud model [60]. System noise temperature ranges between 609 to $676 \mathrm{~K}$ within the receive band. Filter losses are typical for thin film ceramic filters with the passband and stopband characteristics required. LNA gain and noise figure are typical of a $0.13-\mu \mathrm{m}$ GaAs HEMT device. An 8-channel 0.18- 
$\mu \mathrm{m}$ SiGe BiCMOS RFIC containing 6-bit phase shifters and 5-bit attenuators has been demonstrated with the gain and noise figure listed in the table [62].

Board losses assume realistic PCB trace lengths and losses.

Figure 3.4.3 Computation of G/T and EIRP for a X/Ku-band SATCOM array application. Key input parameters are highlighted in yellow, and the results are highlighted in blue. In this example, the array consists of 6400 elements and achieves a $\mathrm{G} / \mathrm{T}$ of $9 \mathrm{~dB} / \mathrm{K}$ and EIRP of $56 \mathrm{dBW}$ at a scan limit of 45 degrees with roughly $1-2 \mathrm{~dB}$ of margin. The given sidelobe control loss reflects a Taylor taper which provides $25 \mathrm{~dB}$ sidelobe suppression

Figure 4.1.1 Visualization of a bill of material cost breakdown for an $\mathrm{X} / \mathrm{Ku}$-band fullduplex satellite communications array in low, medium, and high volume production.

Figure 4.2.1 The parameterized prior unit cell antenna model constructed in CST Microwave Studio. The hybrid appears in the stackup, but is modeled and simulated separately in Agilent ADS using Momentum. Drawing is not to scale

Figure 4.2.2 The prior unit cell antenna design constructed in CST Microwave Studio illustrating (a) stackup layers with ports and feeds, (b) full stackup, (c) substrates only, (d) prepregs only, (e) metallic layers only, (f) waveguide ports, and $(\mathrm{g})$ feed probe vias.

Figure 4.2.3 (a-b) Two views of the prior unit cell antenna model constructed in CST Microwave Studio and (c) an x-ray image of the fabricated unit cell antenna within a multilayer PCB. The x-ray image also includes the single stage hybrid as well as transitions and traces from other layers in the stackup.

Figure 4.2.4 The parameterized model of the prior single-stage branchline coupler design illustrating (a) the hybrid portion of the stackup, (b) the ADS schematic used to optimize the design, and (c) the meshed ADS layout. The transition vias between the hybrid and antenna are not included in the model.

Figure 4.3.1 The unit cell antenna topology consists of an antenna layer, feed layer, and beamforming layer. The antenna layer contains the first two metal layers in the stackup as well as dielectric substrates. The feed layer contains the next four metal layers. The beamforming layer contains three more layers for the 
hybrid and a few other layers for Wilkinson power splitters and subarray-level signal routing. This work includes only the hybrid portion of the beamforming layer.

Figure 4.3.2 The proposed preliminary unit cell antenna model constructed in CST Microwave Studio. The hybrid appears in the stackup, but is modeled and simulated separately in Agilent ADS using Momentum. Drawing is not to scale..

Figure 4.3.3 The preliminary unit cell antenna design constructed in CST Microwave Studio illustrating (a) all six metal layers, (b) stackup layers with ports and feeds, (c) substrates only, (d) prepregs and bond plies only, and (e) metallic layers only. The stripline feed region is enclosed with PEC walls to increase isolation between adjacent feeds and to suppress parallel plate modes. Two sides of the wall are bifurcated to accommodate waveguide ports. Accurate via structures will replace the PEC wall in the final design.

Figure 4.3.4 Port reflection coefficients for the preliminary and prior designs. Both designs are reasonably well matched for this application. The preliminary design has an average of roughly $2 \mathrm{~dB}$ better return loss than the prior design. .73

Figure 4.3.5 Comparison of boresight gain and total efficiency versus frequency for the preliminary and prior designs. Notice the preliminary design improves boresight gain by roughly $1 \mathrm{~dB}$ across the operational band. Both designs exhibit similar efficiency; the preliminary design is slightly more efficient in the lower band and slightly less efficient in the upper band.

Figure 4.3.6 Comparison of $2 \mathrm{D}$ theta cuts of the 3D radiation patterns. Plots on the left column - (a), (c), and (e)—correspond to the prior design. Plots on the right column - (b), (d), and (f) — correspond to the preliminary design. The top, middle, and bottom rows correspond to $\phi=0^{\circ}, 45^{\circ}, 90^{\circ}$, respectively. Curves for seven frequencies are given on each plot, uniformly spaced from 10.5 to 16.5 GHz. The preliminary design overcomes the slight non-symmetry, steep gain roll-off versus frequency, and lower peak gain exhibited by the prior design.

Figure 4.3.7 Comparison of port-to-port coupling and cross-polarization level of the preliminary and prior design. The preliminary design reduces coupling by an 
average of roughly $10 \mathrm{~dB}$ and reduces cross-polarization level by an average of $3 \mathrm{~dB}$. Coupling is less than $-17 \mathrm{~dB}$, and cross-polarization level is less than $-15 \mathrm{~dB}$ across the operating band.

Figure 4.3.8 Comparison of grating-lobe free scan limit of the preliminary antenna design and the prior design. Grating-lobe free scan range is related to the dimensions of the unit cell. The proposed design utilizes a $19 \%$ smaller area and consequently has an extended scan range. The design allows for $47.5^{\circ}$ gratinglobe free scan range at $14.5 \mathrm{GHz}$, which includes a buffer of $2.5^{\circ}$ to ensure the entire grating lobe of a large array does not enter real space.

Figure 4.3.9 Three different branchline hybrid coupler models are constructed: (a) ADS schematic computed using a linear circuit simulator, (b) Momentum layout computed using the method of moments (MoM), and (c) Microwave Studio 3D model computed using the finite-difference time-domain (FDTD) method. ....... 77

Figure 4.3.10 S-parameters of the preliminary dual-stage branchline hybrid coupler compared to the single-stage hybrid coupler of the prior design. The preliminary design exhibits return loss greater than $25 \mathrm{~dB}$, insertion loss lower than $0.3 \mathrm{~dB}$, RMS phase error of 0.188 degrees, and RMS amplitude error of $0.113 \mathrm{~dB}$.

Figure 4.3.11 Comparison of branchline hybrid coupler s-parameters computed using three different simulation tools: circuit model in ADS, 2D layout model in Momentum, and 3D model in CST Microwave Studio. All three simulations compare favorably. All three models are illustrated in Figure 4.3.9......

Figure 4.3.12 Monte Carlo analysis results comparison for the proposed and prior antenna designs for (a-f) 50 simulation runs varying material properties only and (g-l) 50 additional simulation runs varying material properties and applying manufacturing process tolerances. The top, middle, and bottom rows represent $|\mathrm{S} 11|,|\mathrm{S} 22|$, and $|\mathrm{S} 12|$, respectively. Although both designs are reasonably stable, the proposed design exhibits less variation and overall better performance.

Figure 4.3.13 Monte Carlo analysis results comparison for the proposed and prior antenna designs for (a-b) 50 simulation runs varying material properties only and (c-d) 50 additional simulation runs varying material properties and applying 
manufacturing process tolerances. The left column represents the prior design, and the right column represents the proposed design. Co-pol gain for the prior design varies dramatically below $12 \mathrm{GHz}$. Co-pol gain for the proposed design is stable with a peak-to-peak variation of $0.6 \mathrm{~dB}$. Cross-pol gain variation is less than $4 \mathrm{~dB}$ for the proposed design compared to $6 \mathrm{~dB}$ for the prior design.

Figure 4.3.14 Monte Carlo analysis results comparison for the proposed and prior antenna designs for (a-f) 50 simulation runs varying material properties only and (g-l) 50 additional simulation runs varying material properties and applying manufacturing process tolerances. The top, middle, and bottom rows correspond to $10.5,12.5$, and $14.5 \mathrm{GHz}$, respectively. Theta cuts for $\phi=$ $0^{\circ}, 45^{\circ}, 90^{\circ}$ are included on each plot, so the spread is related to radiation pattern uniformity. The proposed design is less sensitive to tolerances and overcomes the non-symmetry and lower peak gain exhibited by the prior design.

Figure 4.4.1 Two candidate final design concepts. Design (a) is very similar to the preliminary design, except the hybrid is connected to stripline antenna feeds through a 5-via transition. Notice the non-symmetry of the hybrid design. This slightly degrades hybrid performance. Design (b) eliminates bends and some length from the stripline feeds and allows for a symmetric hybrid structure. For each design, the antenna and hybrid were optimized. Design (b) was selected due to overall superior performance.

Figure 4.4.2 Conceptual overview of the backdrill and backfill processes used to eliminate unwanted via stubs from high frequency interlayer transitions. The unwanted via stubs (red) in (a) are removed in the backdrill process in (b) and are filled with backfill material (green) in (c). The remaining via consists of the desired interlayer section as well as two 1-2 mil via stubs.

Figure 4.4.3 Photographs taken with a digital microscope of a backdrilled via cross-section from the prior antenna design illustrate (a) the backdrilled region with 4 metal layers in view and (b) the interface between the via and backdrilled region. Via stub length reduces from 60 mil to 1.5 mil. The photograph in (a) also illustrates a 3-4 mil misalignment between layers 2 and 3. This via is also 
pictured in the x-ray image and labeled "transition to coupler" in Figure 4.2.3.

For reference, the via diameter is nominally $20 \mathrm{mil}$.

Figure 4.4.4 The hybrid to antenna transition consists of a quasi-coaxial structure with a center via and five surrounding ground vias. To eliminate parasitic capacitance, the remaining via stubs on top and bottom are backdrilled and backfilled with a material that has similar properties to the substrates.

Figure 4.4.5 Port-to-port isolation improves from $17 \mathrm{~dB}$ to $22 \mathrm{~dB}$ and cross-polarization level improves from $-15 \mathrm{~dB}$ to $-17 \mathrm{~dB}$ by adding a ground via between ports and maximizing the distance between the tips of the feed forks. .90

Figure 4.4.6 ADS models for two hybrid designs corresponding to the two topologies presented in Figure 4.4.1. The schematics are pictured in (a) and (d), meshed layouts in (b) and (e), and surface currents in (c) and (f). The second design in (d-f) exhibits best performance.

Figure 4.4.7 Comparison of port reflection coefficients for the final, preliminary, and prior designs. Reflection coefficients are plotted (a) in rectangular form and (b) on a Smith chart. The final design exhibits slightly degraded port 1 return loss in the lower portion of the band.

Figure 4.4.8 Comparison of port-to-port coupling and cross-polarization level of the final, preliminary, and prior designs. Both parameters have improved by an average of roughly $10 \mathrm{~dB}$ across the operating bandwidth. Coupling is less than -22 $\mathrm{dB}$, and cross-polarization level is less than $-17 \mathrm{~dB}$.

Figure 4.4.9 Comparison of gain and efficiency for the final, preliminary, and prior designs. The final design achieves an increase in gain of roughly $2 \mathrm{~dB}$ relative to the prior design.

Figure 4.4.10 Comparison of 2D theta cuts of the 3D radiation patterns. Plots on the left, middle, and right columns correspond to the prior, preliminary, and final designs. The top, middle, and bottom rows correspond to $\phi=0^{\circ}, 45^{\circ}$, and $90^{\circ}$, respectively. Curves for seven frequencies are given on each plot, uniformly spaced from 10.5 to $16.5 \mathrm{GHz}$. The final design represents an improvement over the preliminary design in peak gain and gain uniformity. 
Figure 4.4.11 S-parameters of the preliminary and final hybrid designs computed in Microwave Studio. The final design has slightly degraded performance due to the addition of via transitions, but still meets requirements. Insertion loss is less than $0.4 \mathrm{~dB}$, RMS phase error is 0.628 degrees, and RMS amplitude error is $0.156 \mathrm{~dB}$. .96

Figure 4.4.12 Two simulation approaches compare favorably. (a) A small phase error is observed in port reflection coefficients which appears as a horizontal shift between the two curves. The combined model exhibits roughly 2-4 dB higher coupling between ports.

Figure 4.4.13 A 64-element array of unit cell antennas configured in an equilateral triangular lattice was constructed to demonstrate successful integration and operation of the unit cell antenna design in an array environment. .98

Figure 4.4.14 The computed 3D radiation pattern for a 64-element array using a $22 \mathrm{~dB}$ Taylor amplitude taper exhibits a realized gain of $22.2 \mathrm{dBi}$ at $14 \mathrm{GHz}$. This compares favorably to the expected realized gain which can be approximated as the element realized gain increased by the array gain and decreased by the amplitude taper loss; i.e., $6.3 \mathrm{dBi}+10 \log 64 \mathrm{~dB}-1.8 \mathrm{~dB}=22.6 \mathrm{dBi}$.

Figure 5.2.1 The current state-of-the-art in tile-based array implementation. Pictured here is a 512-channel X-band ISR subarray with integrated SiGe beamforming RFICs and GaAs LNAs. The subarray and control PCBs are mated together with an aluminum frame. The overall thickness is less than 0.5 inches.

Figure 5.2.2 Micro-fabricated microwave structures are expected to revolutionize tile-type array implementations over the next two decades. Pictured here are (a) the physical geometry of an air-core rectangular coaxial transmission line from [85], (b) a micro-fabricated branchline coupler operating at $60 \mathrm{GHz}$ with ground-signal-ground probe interfaces from [86], (c) a 4-element array operating at $94 \mathrm{GHz}$ using a cavity-backed patch antenna with $4.1 \%$ bandwidth and $8.3 \mathrm{dBi}$ gain at 95\% efficiency from [87], and (d) a slot array operating from 87 to $102 \mathrm{GHz}$ achieving $14 \mathrm{~dB}$ gain at $94 \mathrm{GHz}$ and 30 degree scan angle over a $15 \mathrm{GHz}$ bandwidth from [88]. (All images: (C IEEE) 104 


\section{Chapter 1}

\section{Introduction}

\subsection{Phased Arrays}

Electromagnetic phased array antennas or phased arrays consist of a plurality of antenna structures that each coherently radiate or receive electromagnetic energy, and when properly phased or time-delayed, interfere constructively in desired spatial regions as conceptualized in Figure 1.1.1. This work proposes a unit cell antenna structure for phased arrays operating within the super high frequency (SHF) band from 3-30 GHz.

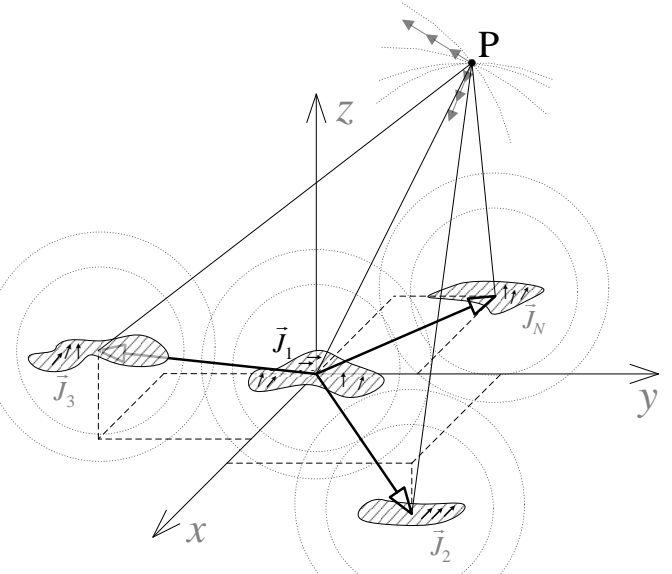

Figure 1.1.1 A general concept of a phased array antenna illustrates radiation from source current distributions $\vec{J}_{i}$ oriented in space and phased in time such that radiated fields constructively interfere in the desired direction of point $P$. 
The components responsible for amplification, filtering, and phase or time delays are collectively referred to as the beamformer in a phased array. The interference pattern, or beam pattern, can be manipulated using well known synthesis and optimization techniques that are widely studied and applied in undergraduate electrical engineering courses on discrete-time signal processing. In this way, nearly every undergraduate in the broader field of electrical engineering has exposure to the mathematical framework of phased array theory, where in this case, a spatial filter is created by summing complexly weighted spatial samples within the array aperture analogous to a discrete-time filter that is created by summing complexly weighted time-domain samples within the window of its impulse response [1].

The financial advantage of implementing phased arrays using printed circuit board (PCB) and semiconductor manufacturing processes has prompted the phased array community to gravitate toward surface mount technologies and tile-type array implementations, particularly when minimized profile and maximized channel density are desired. The tile architecture evolves handin-hand with advancements in highly integrated beamforming RF integrated circuits, multi-chip modules, micro-fabricated or RF MEMS devices, and 3D packaging technologies. Intelligence, surveillance, and reconnaissance (ISR) sensors, low-cost radar, lightweight airborne and spaceborne arrays, and mobile satellite communications are some important applications that benefit from the tile architecture.

To satisfy manufacturing constraints of PCB processes and simplify distribution of power, control, and RF feed networks, phased arrays with large physical apertures are commonly partitioned into subarrays. The selection of subarray size, number of elements, and placement within the aperture are degrees of freedom available to the designer. Each subarray consists of unit cell antenna elements as illustrated in Figure 1.1.2. 


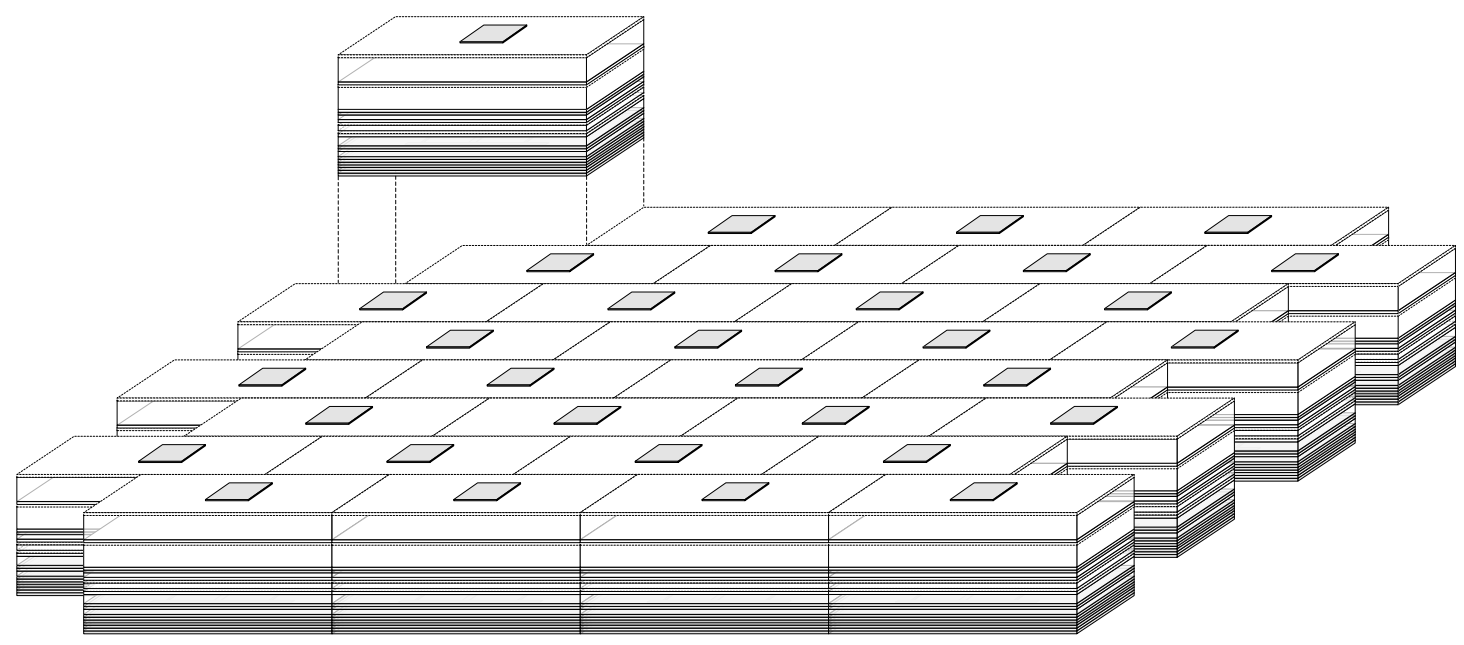

Figure 1.1.2 Planar phased arrays implemented as multilayer PCBs are comprised of many unit cell antennas, combiners, and solder attached beamforming electronics. One primary challenge is to design an electromagnetically robust structure that yields a well-behaved radiation pattern in a dynamic environment and can withstand variation associated with PCB materials and manufacturing process tolerances.

The antenna elements are typically placed in a grid or lattice formation along the aperture plane. Other non-planar apertures or piecewise conformal arrays can be constructed using a plurality of planar subarrays or flexible substrates and thus still benefit from low-cost PCB processes. Conformal arrays structured in this way are useful in applications where extended scan range or mechanical compliance to fixed form factor is required.

The unit cell of a phased array refers to the elementary building block from which the entire phased array antenna is constructed. The size of the unit cell represents the distance between discrete aperture samples and dictates the scan volume in which the array will not generate grating lobes, artifacts of an under-sampled aperture.

In addition to the radiating element, a unit cell consists of front-end amplifiers, filters, and beamforming electronics. A generalized analog full-duplex front-end is provided in Figure 1.1.3. Each antenna receives and transmits two orthogonally polarized electromagnetic waves. Switches or filters provide beam or channel selectivity. An amplification stage is followed by phase shifters or time delays, variable attenuators, and coherent summing networks. Controlling amplitude and 
phase shift (or time delay for large aperture or wideband arrays) of each channel implements a coherent weighted sum used to electronically reconfigure the far-field radiation pattern (i.e., to form beams or place nulls). As the size of the aperture and the number of antennas increase, beamwidth narrows and directivity increases. To reduce overall system cost, many of the components can be consolidated into integrated circuits or multi-chip modules and collocated on multilayer PCBs. Further reduction in system cost can be achieved through the utilization of lowcost processes and technology nodes for consolidation of beamforming elements. Higher component consolidation allows for reduction in unit cell size, and consequently, extension of grating-lobe free scan volume or maximum operational frequency. Scan volume and operational bandwidth are two important figures of merit for a phased array.

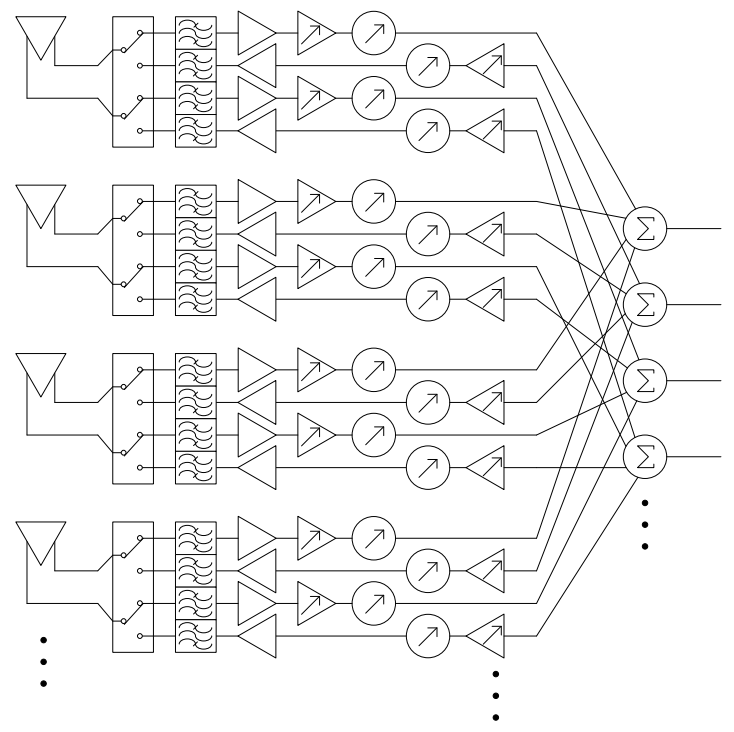

Figure 1.1.3 A generalized analog full-duplex phased array front end consists of antenna elements, band or beam switches, filters, variable amplifiers, phase shifters or time delay units, and coherent summers. This paper describes the flexibility offered by such a front end and focuses on the antenna element design.

\subsection{Objective}

The primary goal of this work is to produce a unit cell antenna design using systematic microwave design principles and modern software analysis tools for a full-duplex shared aperture 
$\mathrm{X} / \mathrm{Ku}$-band satellite communications array application. The unit cell must represent an improvement in scan range, bandwidth, gain, and manufacturability over an existing design produced on a prior effort. Additionally, the design must be generally extensible to phased array implementations across the SHF band.

\subsection{Methods}

Solutions to microwave circuit design problems are generally initiated in a circuit simulator. A circuit simulator computes s-parameters based on pre-compiled or closed form relationships, providing the designer will valuable feedback with very minimal simulation run time. The circuit simulator utilized in this work is Advanced Design System (ADS), version 2011, developed by Agilent Technologies.

Upon successful implementation in a circuit simulator, the designer often transitions to a more accurate electromagnetic design tool which computes Maxwell's equations for user-defined excitation sources, structures, materials, and boundary conditions. When switching tools, the designer aims to reproduce results computed in a circuit simulator or gain insight into the causes for disparities between simulation domains. The design tool employed in this work is Microwave Studio, version 2011, developed by Computer Simulation Technology (CST).

To realize aggressive antenna performance targets, the design process also requires assimilation of knowledge from Literature and iterative experimentation with novel techniques to overcome performance deficiencies of the prior design. In the preliminary design phase, simplified electromagnetic models are constructed for promising antenna candidates. To facilitate trade studies, these models are parameterized such that all key dimensions and material properties are variables within the design environment. A practiced designer first understands the primary radiating mechanism of a candidate structure and cleverly assigns variables and initial conditions to achieve an expected result. Then, the designer performs a parametric study and observes trends 
and sensitivities. In this work, the overall unit cell is divided into separate preliminary designs for the antenna and hybrid. Inputs are implemented as non-realizable waveguide ports, structures are void of detailed features, substrates in the stackup are combined to reduce the number of unique layers, and the simulation mesh is rough. These measures are taken to reduce simulation time and maximize design efficiency.

Once the designer understands performance and has iteratively converged on a promising initial design, a more resolved model is constructed. In this PCB-based design, this step requires an understanding of PCB materials and manufacturing processes. A complete stackup that can be successfully manufactured replaces the preliminary dielectrics, and parameters are defined and bounded according to commercially available materials. Model structures include finer details, optimal miters replace stripline bends, rounded vias with pads replace square pillars, conductor layer thickness is adjusted according to the lamination and plating processes required to manufacture the stackup, substrates and conductors are assigned realistic physical properties, and testable interfaces replace non-realizable excitation ports. Simulation results are assessed and the designs are optimized using insight gained from preliminary design studies. Once a final design achieves the objectives, manufacturability is assessed and reviewed with the manufacturer to verify compliance to design rules.

In this work, simulated data for both preliminary and final designs are presented for the proposed design and the prior design. Additionally, a Monte Carlo investigation of both designs is employed to compare susceptibility to materials and manufacturing process tolerances. Data that is generated in Microwave Studio or ADS is exported as a touchstone s-parameter file and processed in MATLAB.

\subsection{Nomenclature}

Key variables, constants, and units relevant to this paper are provided in this section. 


\subsubsection{Variables}

\begin{tabular}{clcl}
\hline Symbol & Meaning & Symbol & Meaning \\
\hline$\alpha$ & complex weight & $\theta$ & elevation angle \\
$\mathrm{d}$ & interelement spacing in x & $\phi$ & azimuth angle \\
$\mathrm{b}$ & interelement spacing in y & $\mu$ & permeability \\
$\gamma$ & lattice angle & $\varepsilon$ & permittivity \\
$\psi$ & scalar parameter & $\eta$ & intrinsic impedance \\
$\xi$ & dummy variable & $\rho$ & charge density \\
$\lambda$ & wavelength & $\omega$ & angular frequency \\
$\sigma$ & conductivity & $\partial$ & partial differential \\
$A$ & complex peak amplitude & $\vec{A}$ & magnetic vector potential \\
$\vec{E}_{\text {array }}$ & array electric field intensity & $\vec{H}$ & magnetic field intensity \\
$\vec{G}_{\text {array }}$ & array radiation pattern & $\vec{E}$ & electric field intensity \\
$\vec{e}$ & active element electric field & $\vec{J}$ & electric current distribution \\
$\vec{S}$ & Poynting vector & $\Phi$ & electric scalar potential \\
$\overrightarrow{\mathrm{D}}$ & complex directivity & $\mathrm{D}$ & directivity \\
BW & beamwidth & $\mathrm{B}$ & bandwidth \\
\hline
\end{tabular}

\subsubsection{Constants}

\begin{tabular}{llll}
\hline Symbol & Value & Units & Description \\
\hline $\mathrm{c}$ & 299792458 & meters per second & speed of light in free space \\
$\mu_{0}$ & $4 \pi \times 10^{-7}$ & henries per meter & permeability of free space \\
$\varepsilon_{0}$ & $8.854 \times 10^{-12}$ & farads per meter & permittivity of free space \\
$\eta_{0}$ & $376.7 \approx 120 \pi$ & ohms & intrinsic impedance of free space \\
\hline
\end{tabular}

\subsubsection{Units}

\begin{tabular}{llll}
\hline Unit & Description & Unit & Description \\
\hline $\mathrm{dB}$ & decibels & $\mathrm{dBd}$ & decibels, normalized to dipole \\
$\mathrm{dBi}$ & decibels, normalized to isotropic & $\mathrm{dBic}$ & decibels, circular polarization \\
\hline
\end{tabular}




\section{Chapter 2}

\section{Background}

\subsection{Historical Achievements Preceding the Reflector Antenna}

To appreciate the advantages offered by the phased array antenna and the merits of the implementation proposed in this work, it is helpful to understand the developments leading up to the invention of the phased array antenna.

Classical Greek mathematicians of the last few centuries BC were interested in geometrical objects and their properties. The elders of antiquity, including Thales, Pythagoras, Democritus, and Eudoxus, collectively unearthed a wealth of information that culminated in Euclid's capstone work Elements (c. 300 BC). In this work, Euclid employs a set of intuitive definitions or axioms and rigorously deduces and proves theorems. His logical methods of proof and the formalism of his prose influenced mathematicians for the next two millennia.

Building upon Euclid's work during the productive Hellenistic period following the conquest of Alexander the Great, Greek mathematicians discovered very interesting properties of 
geometrical curves formed by the intersection of a plane and a right angle cone, known as "conic sections." Most notably, Archimedes (c. 287-212 BC) applied Euclidean methods and theorems of conic sections to solve practical engineering problems, and Apollonius of Perga (c. 262-190 BC) superseded the work of his predecessors with innovative terminology and methodologies for analyzing problems in his eight-volume treatise Conics where he introduces for the first time the terms ellipse, parabola, and hyperbola. A pupil of Apollonius, Diocles (c. 240-180 BC), is given credit for describing the essential elements of the focus and directivity, although the terms focus and directivity were not introduced until the work of Pappus of Alexandria (c. 290-350 AD).

Diocles first demonstrated that lines parallel to the axis of symmetry for a paraboloid converge at a single point assuming the lines are reflected by the interior of the paraboloid. Figure 2.1.1 illustrates the formation of a parabola as an intersection of a plane with a double cone and the property of the focus in the context of Euclidean geometry. It is fabled ${ }^{1}$ that Archimedes leveraged this property of the parabola by instructing Syracusian soldiers to position reflective sheaths in the shape of a parabola so that reflected sunlight would focus and ignite the tar-laden wooden hulls of enemy Roman ships during the Siege of Syracuse (c. 214-212 BC), a battle in which Archimedes was a casualty [2].

\footnotetext{
${ }^{1}$ The feasibility of this approach was later verified in 2005 when MIT students ignited a replica Roman ship with reflected sunlight from 127 one square foot mirrors positioned approximately in the shape of a parabola [84].
} 


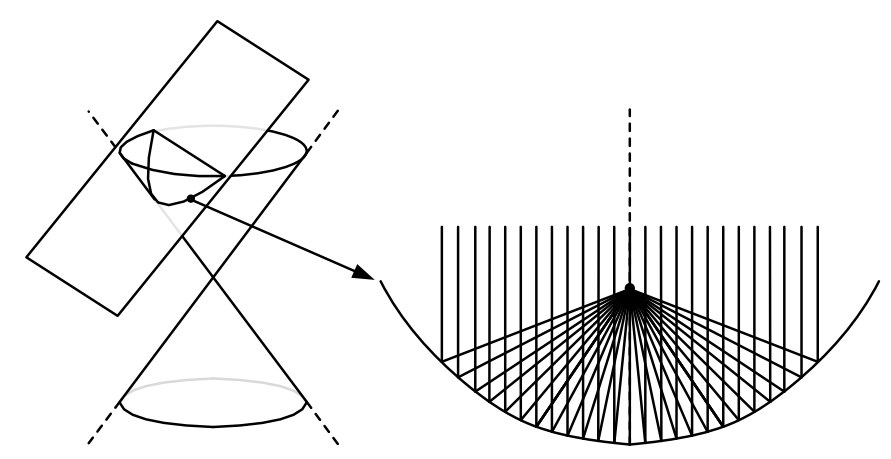

Figure 2.1.1 Parabolic curve represented as a conic section illustrating the concept of the focus in the context of Euclidean geometry.

In the $3^{\text {rd }}$ century $\mathrm{AD}$, one of the last Greek mathematicians from the Hellenistic period, Diophantus (c. 201-284), developed the methods and notation of algebra similar in form to ancient Babylonian mathematicians and compiled many algebraic problems with equations and numerical solutions in his work, Arithmetica (c. 250). For this work, he has been deemed the "Father of Algebra."

Later that century, Pappus of Alexandria (c. 290-350) produced an eight-volume piece on Euclidean geometry and the conics, Collection (c. 340), which further explored prior works of Euclid, Archimedes, and Apollonius. The most influential mathematician progressing toward analytical geometry and modern forms of algebra and trigonometry may be Muhammad ibn Musa al-Khwarizmi (c. 780-850) and his work, The Compendious Book on Calculation by Completion and Balancing (c. 830). Other than advancements in algebra and trigonometry, most notably by al-Khwarizmi and other Persian mathematicians, the pace of progress in Mathematics throughout the Middle Ages was relatively modest [3].

It was not until the $17^{\text {th }}$ century when Rene Descartes (1596-1650) bridged the gap between Euclidean geometry and algebra in La Geometrie (1637). Unlike Euclidean geometry, analytical or Cartesian geometry establishes a coordinate system a priori (including negative numbers) to define problems and employs methods of algebra to produce solutions. This marked a critical juncture in the road leading to infinitesimal calculus, and of greatest significance, the 
mathematical framework capable of solving differential equations. Figure 2.1.2 depicts a parabola in the context of Cartesian geometry, where the curve represents the solution to an equation consisting of numerical constants and the ordinate and abscissa of the coordinate system. Pioneers of the European scientific revolution assimilated the constructs of analytical geometry and swiftly advanced mathematics along with many other sciences in the seventeenth and eighteenth centuries.

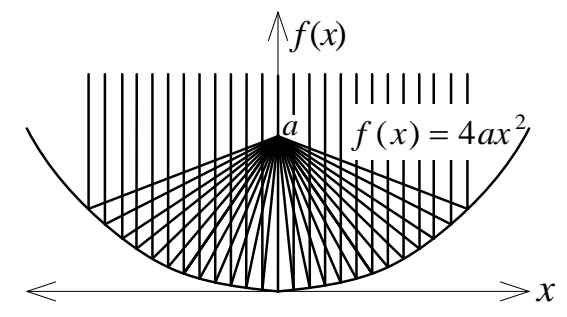

Figure 2.1.2 Parabolic curve illustrating the concept of the focus in the context of Cartesian geometry.

During this productive period, scientists identified many applications exploiting the useful properties of conics. Most relevant to this discussion is the reflecting telescope invented by Isaac Newton in 1668 and later presented to the Royal Society in 1672. In Newton's design, the objective consists of reflecting mirrors instead of refracting lenses. Prior to this invention, Galilean and Keplerian telescopes positioned two refracting lenses at the objective and eyepiece to focus light emanating from distant objects. Although these designs more efficiently captured $\operatorname{light}^{2}$ for a fixed aperture size, images were significantly degraded by chromatic and spherical aberration. Newton decided to accept spherical aberration associated with a spherical reflector to avoid a more complicated manufacturing process of a parabolic shape, but his reflecting objective successfully eliminated chromatic aberration and marked a noteworthy achievement of practical importance for astronomers.

${ }^{2}$ Due to the poor reflectivity of speculum metal mirrors manufactured during this time period which produced a reflectivity of $68 \%$ at a wavelength of $6000 \AA$ [105]. 
As speculum metal (i.e. copper/tin alloy) manufacturing processes matured, other types of telescopes utilizing parabolic, elliptic, or hyperbolic reflectors to minimize spherical aberration were popularized, including those described by James Gregory and Laurent Cassegrain in the 1670s. A summary of the primary topologies are illustrated in Figure 2.1.3. In each case, the properties of conics are applied to manipulate incident light so that images of distant objects are viewable by the observing eye.

Further improvements in reflector manufacturing processes in the mid- $19^{\text {th }}$ century, including the deposition of thin silver or aluminum films onto silicon dioxide glass, produced increased reflectivity and decreased oxidation-induced optical aberration. For these reasons, reflection type telescopes are prevalent in modern applications.

The James Webb Space Telescope ${ }^{3}$ illustrated in Figure 2.1.4, for example, demonstrates the profound engineering and manufacturing advancements of the $21^{\text {st }}$ century, but employs the same fundamental theory of operation originating from ancient Greek mathematicians in a configuration first proposed in a journal submission from a secondary school instructor and priest, Laurent Cassegrain (1629-1693) in 1672 [4].

\footnotetext{
${ }^{3}$ The JWST is an international infrared (IR) observatory led by NASA, capable of observing wavelengths from 0.6 to $29 \mu \mathrm{m}$. The Korsch-style [72] Cassegrain telescope consists of a $6.6 \mathrm{~m}$ hexagonally-sectioned primary reflector shaped as a $131.4 \mathrm{~m}$ paraboloid, a single $0.75 \mathrm{~m}$ secondary reflector in the shape of a convex hyperboloid, and a tertiary reflector to minimize astigmatism, coma, and spherical aberration. Mirrors are gold-plated beryllium with RMS surface figure error of $24 \mathrm{~nm}$ and position tolerance of +/- 0.1 $\mathrm{mm}$ [61]. The satellite will launch and transfer to an earth-sun Lagrange point (L2) and be shielded from IR emissions of Earth, Earth's moon, and the Sun. The mirrors operate at $50 \mathrm{~K}$, and its coolest instrument is cooled with liquid helium to $7 \mathrm{~K}$. An angular resolution of 0.1 arc-seconds at a 2 micron wavelength will enable the study of birth and evolution of galaxies and the formation of stars and planets [78].
} 

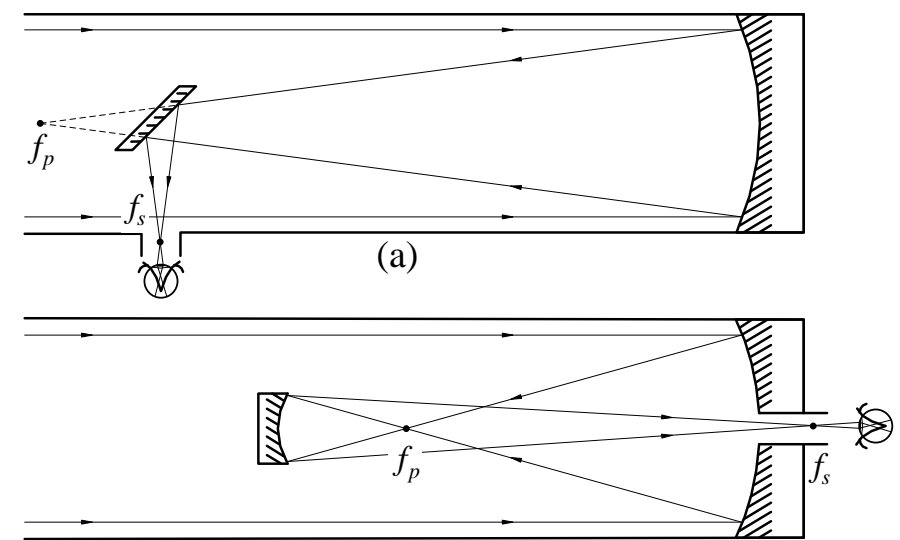

(b)

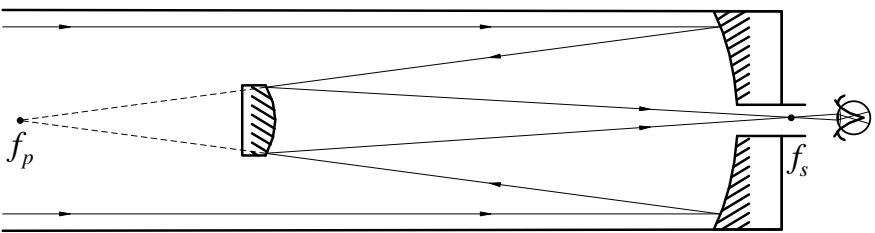

(c)

Figure 2.1.3 Reflective telescope designs popularized by (a) Isaac Newton, (b) James Gregory, and (c) Laurent Cassegrain [5].

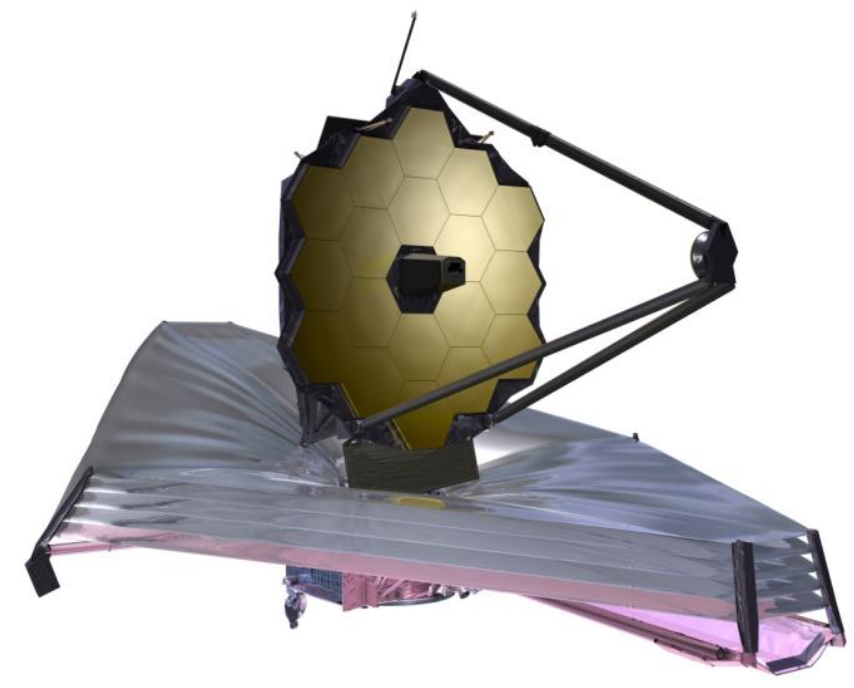

Figure 2.1.4 The James Webb Space Telescope (JWST) satellite system depicting its Cassegrain three-mirror anastigmatic optical system and thermal shields [6]. Images courtesy of NASA.

Renewed interest in optics and astronomy in the mid to late $19^{\text {th }}$ century coincided with arguably the most important advancement in science, the unification of separate laws relating to electricity and magnetism into electromagnetic theory [7] and the prediction that "light itself [...] 
is an electromagnetic disturbance in the form of waves propagated through the electromagnetic field according to electromagnetic laws" by Scottish Physicist James Clerk Maxwell (1831-1879) $[8]$.

Maxwell's proposed theory prompted international investigation, validation, and application of electromagnetic theory from both mathematical and physical perspectives. Early investigations focused on experimentation with visible light due to the availability of tools and a wealth of knowledge accumulated after 200 years of productive optics and astronomy progress during the scientific revolution. Maxwell himself produced the first colored photograph of a tartan patterned ribbon and thereby established red, green, and blue as the primary colors, contrary to popular belief at the time [9].

Investigation of Maxwell's curious propagation medium which permits light propagation, ubiquitously referenced as the luminiferous aether in Maxwell's writings, prompted the famed Michelson-Morley interferometer experiment in 1887 [10] and led Hendrik Lorentz, Henri Poincare, and Albert Einstein to establish the Theory of Special Relativity in 1905 which remains intact after more than a century of accelerated research. Although this work does not assume a luminiferous aether and therefore excludes its existence by Occam's razor, the elegance of Einstein's theory lies in its resemblance to the mathematics of Maxwell's theory of electrodynamics [11].

Later research in the $20^{\text {th }}$ century produced the Quantum Theory. In this regard, Maxwell's contributions ignited the revolution in theoretical physics and lifted the floodgates leading to many useful applications which have profoundly impacted the human experience.

The practical applications most relevant to this discussion pertain to experimental work on electromagnetic wave propagation pioneered by Heinrich Hertz (1857-1894) in Germany, Guglielmo Marconi (1874-1937) in Italy, Alexander Popov (1859-1905) in Russia. However, it 
may be enlightening to first discuss early attempts to understand and demonstrate wave propagation.

Some of the first recommendations for wireless transmission systems were patented by US inventors Mahlon Loomis [12] and William Ward [13] in 1872. Although the underlying principles were not accurately represented and apparatus descriptions were vague, both had realized the gravity of this prospect - useful information can be transferred from a human in one location to a human in another location using some phenomenon associated with electricity.

After inventing the wired telephone in 1876, Alexander Graham Bell and Sumner Tainter patented and demonstrated the photophone in 1880 [14]. In this application sunlight was modulated by voice and beamed to a receiver; the receiver captured sunlight with a parabolic reflector and converted sunlight into a voltage representing the spoken message using photovoltaic selenium cells located at the focus, a precursor to fiber optic communication.

In 1879, David Edward Hughes invented the spark-gap transmitter and successfully transmitted and received Morse code using radio wave propagation over 500 yards [15]. However, his achievement was undeservingly dismissed by the Royal Society as a demonstration of induction and not radio wave propagation, and consequently, his work was largely overshadowed by the more meticulous and rigorous work published shortly thereafter by Heinrich Hertz.

In 1887, Hertz experimentally verified the propagation of electromagnetic waves as predicted qualitatively by Faraday and quantitatively by Maxwell [16] using Hughes' spark-gap transmitter with cleverly designed antennas, including the dipole and the loop antenna. The following year, Hertz invented and demonstrated the world's first parabolic reflector antenna used to transmit and receive electromagnetic waves, confirming Maxwell's proposition by experimentally correlating the observations of radio waves and light [16]. Hertz's apparatus is illustrated in Figure 2.1.5 [17]. Oddly enough, Hertz seemed to lack the business savvy to monetize his profound achievements 
and is famed for his response to questions regarding potential applications, as "Nothing, I guess" [18].

Shortly after Hertz's premature death at age 36, two notable pioneers, Alexander Popov and Guglielmo Marconi, recognized the practical implications of Hertz's discoveries. Popov demonstrated a ship-to-shore radio system spanning 3 miles in 1897, and in the same year, Marconi founded a company dedicated to wireless telegraphy applications. Marconi was awarded a Nobel Prize in 1909 for installing safety communication devices on ships, establishing a transatlantic radio service, and pioneering short wave radio communication in England [19].


Figure 2.1.5 The 455-MHz cylindrical parabolic reflector antenna fed by a dipole antenna and spark-gap transmitter invented by Heinrich Hertz in 1888 [16, 17].

\subsection{The Significance of the Reflector Antenna}

The first instantiation of a parabolic reflector antenna by Hertz in 1888 marks a significant achievement in the context of this paper. Since its advent, the parabolic reflector antenna has remained a primary resource for radar, communications, and radioastronomy. In 1899, parabolic reflector antennas supported the first terrestrial microwave link to bridge the English Channel, and three years later, the Atlantic Ocean. 
Upon studying an anomalous interference encountered in short-wave transatlantic communication at Bell Labs, Karl Jansky inadvertently discovered an astronomical radio source ${ }^{4}$ and announced his discovery in 1933. Inspired by Jansky's report, Grote Reber, constructed a 9meter parabolic reflector at his home in 1937 and confirmed Jansky's observations. This work inspired an intense international interest in radioastronomy [20].

In Reber's lifetime, hundreds of radio telescopes like the one he constructed in his backyard were deployed around the world ${ }^{5}$. The parabolic reflector remains the cornerstone of modern-day radio telescopes and interferometric radio telescope arrays. In 2012, a major interferometric array project which began in 1972 was renamed the Karl G. Jansky Very Large Array in honor of his discoveries and those of subsequent pioneers, including Grote Reber, J. S. Hey, J. Oort, H. van der Hulst, M. Purcell, and H. Ewen [21, 22].
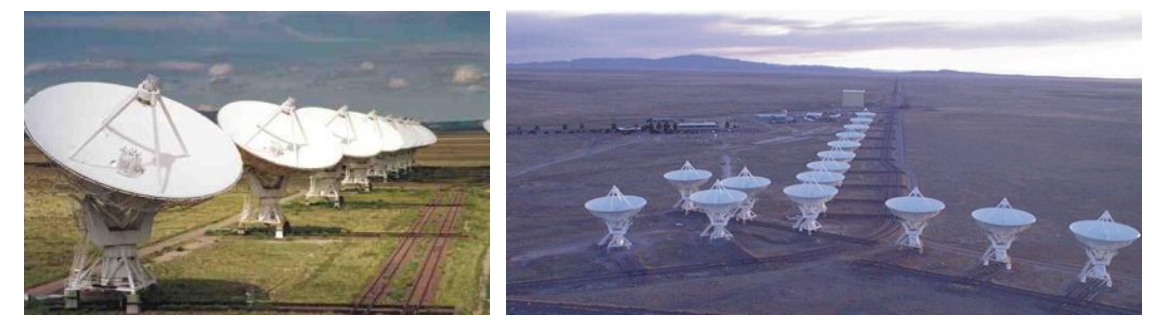

Figure 2.2.1 The Karl G. Jansky Very Large Array (VLA) radio telescope utilizes 27 different parabolic reflectors that are each 25 meters in diameter to observe galaxies and other astronomical objects. Both photographs were taken by Dave Finley. Image courtesy of NRAO/AUI website at http://www.nrao.edu.

The parabolic reflector antenna maintains a dominant presence in the commercial communications sector largely due to its cost effectiveness. It is often employed as a point-topoint microwave relay in the cellular infrastructure or as a terminal communicating with direct

\footnotetext{
${ }^{4}$ Jansky observed radio waves emanating from the constellation known as Sagittarius in the direction of the center of the Milky Way galaxy.

${ }^{5}$ The Arecibo Observatory in Puerto Rico is currently the largest single-dish radio telescope in operation, a spherical reflector built into a mountain with a diameter of 1001 feet. In this instance, a spherical reflector was selected to allow the beam to scan by means of feed movement without severe degradation that would be associated with a parabolic reflector when the feed strays from the focus [24].
} 
broadcast satellite (DBS) systems in geosynchronous or Molniya orbits. DBS terminals systems consist of an injection molded reflector, low-noise block downconverter, feed, mount, and coaxial cable. These elements are typically offered as a complementary rental with a satellite service subscription. Even in regions of severe economic depression, dish antennas ubiquitously adorn the landscape as reflected in Figure 2.2.2.

In Mexico, for example, the asset-based poverty rate [23] in 2012 is an estimated 47\% [24]. In other words, roughly one of two households is below the poverty line. Nonetheless, over 6 million subscribers, or roughly 1 in 5 households, pays a monthly service fee for access to hundreds of television channels, and this number is growing. It is anticipated that telecommunications will continue to maintain profitability for at least the next decade in Mexico. Recent financial statements of Mexico's leading service providers reiterate this bullish forecast; first quarter sector revenue increased by $11.9 \%$ year-over-year in 2012 [25].

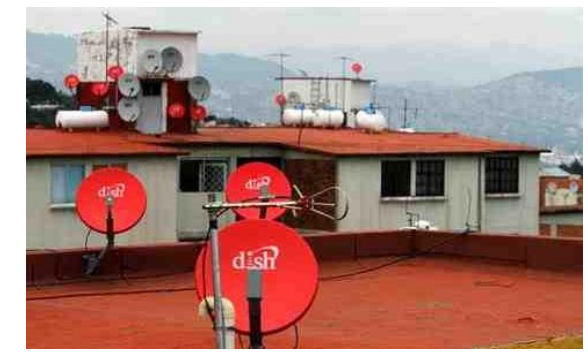

Figure 2.2.2 Colorful satellite dish antennas adorn landscapes in cities around the world. This photograph was taken by Carlos Jasso of Reuters in Mexico City on July 12, 2011. It reflects the prevalence of media access in even the poorest of developing nations. The reflector antenna serves as a cost-effective solution for this application.

High gain parabolic reflectors are also mounted on nearly every satellite to relay mission data to earth terminals or broadcast information to users. For example, the next generation narrowband satellite communications system for the United States, known as the Mobile User Objective System (MUOS), utilizes two deployable mesh reflectors on each satellite and large reflectors at 
each earth terminal to direct radio traffic between the terrestrial network and globally-dispersed users, illustrated in Figure 2.2.3.

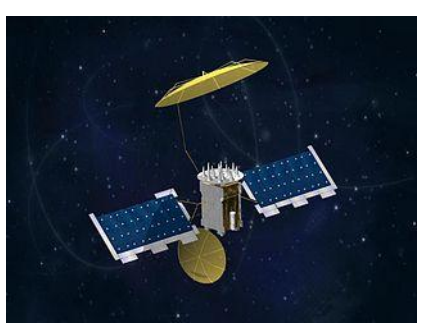

(a)

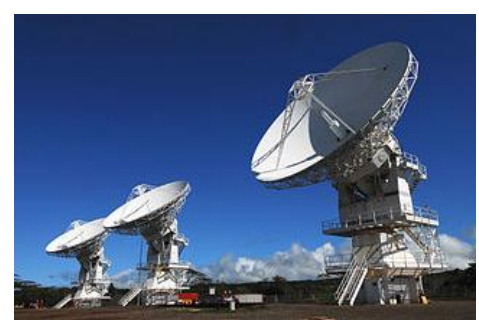

(b)

Figure 2.2.3 The Mobile User Objective System (MUOS) is the next generation narrowband communications system for the United States. (a) Each satellite relies on two deployable gold mesh reflectors which are 5.4 and 14 meters in diameter, and (b) each earth terminal in relies on a reflector antenna that is roughly 20 meters in diameter [26].

\subsection{The Advantages of the Phased Array Antenna}

To understand the advantages and disadvantages of a phased array antenna, it is important to first consider the similarity between the phased array antenna and the continuous aperture antenna (e.g. parabolic reflector). Similarity between the two antennas can be described by invoking the concepts of the Fourier transform and the Nyquist-Shannon sampling theorem.

It can be shown, with appropriate assumptions, that the continuous current distribution associated with a continuous aperture antenna, such as the parabolic reflector antenna, and its corresponding radiation pattern constitute a Fourier transform pair [27, 5, 28]. Qualitatively, a parabolic antenna is depicted along the bottom axes of Figure 2.3.1. In the case of the receive mode of operation, an incoming plane wave of number $k_{0}=2 \pi / \lambda_{0}$ normal to the parabola's axis of symmetry, represented by lines or rays, is reflected by the continuous parabolic surface and collected by an overhead feed antenna. According to the property of the focus, each ray undergoes an identical time delay or phase shift for fixed frequency. In this way, the feed antenna 
implements a spatial summing node at which reflections coherently combine to form a boresight beam.

The current distribution $f(x)$ of the effective aperture plane, given on the middle set of axes, is uniform over one-dimensional space $x$. The resultant far-field radiation pattern $F(u)$, where $u=k_{0} \sin \theta$, represents the Fourier transform of $f(x)$; that is, $F(u) \stackrel{\mathfrak{J}}{\Leftrightarrow} f(x)$.

Similarly, when extended to two dimensions, $F(u, v) \stackrel{\Im}{\Leftrightarrow} f(x, y)$ where $u=k_{0} \sin \theta \cos \phi$ and $v=k_{0} \sin \theta \sin \phi$. This implies that any non-periodic current distribution in the aperture plane can be synthesized from a continuous distribution of plane waves of varying incidence, or more intuitively, any non-periodic current distribution gives rise to a continuous far-field radiation pattern which varies with spatial direction. A finite current distribution, then, can be likened to a finite impulse response (FIR) filter, where instead of a temporal frequency response, a spatial frequency response is established. 


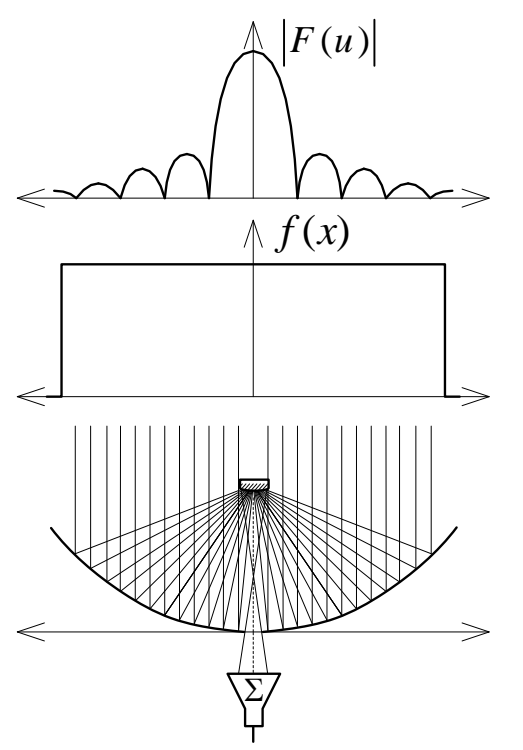

(a)
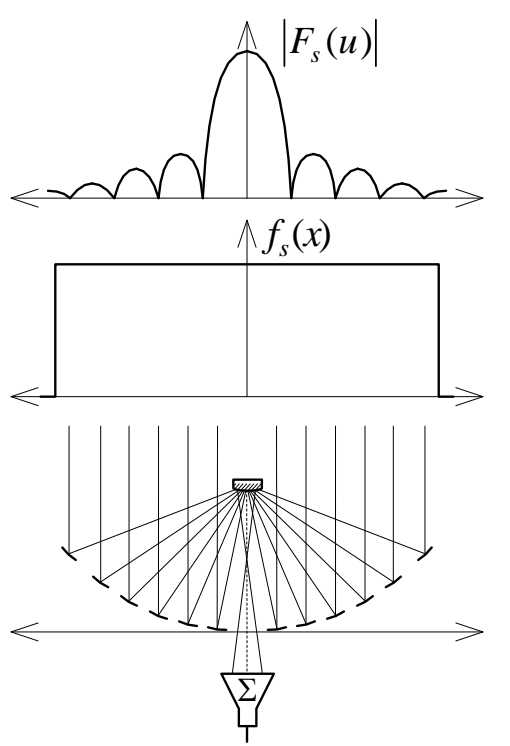

(b)

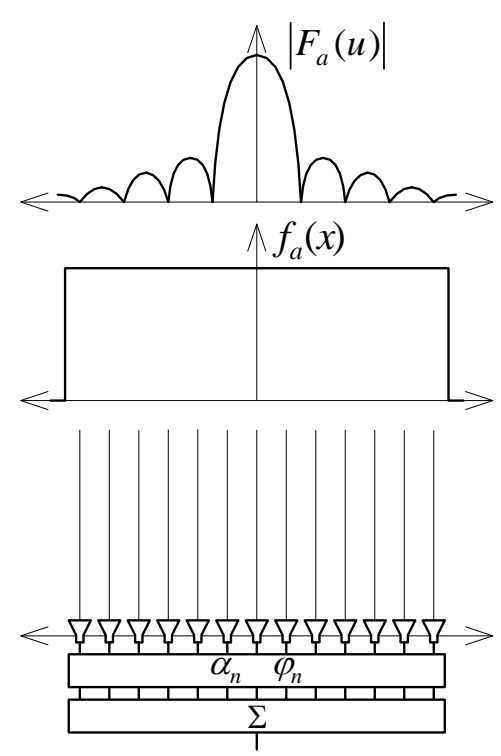

(c)

Figure 2.3.1 Qualitative depiction of the similarities between (a) the parabolic reflector antenna and (c) the phased array antenna. A parabolic reflector antenna is represented as a continuous aperture relying on a spatial summing node. A phased array is represented as a sampled aperture where samples are complexly weighted and summed using beamforming electronics.

The illustration in Figure 2.3.1 (b) represents a discretization of the continuous current distribution established by the continuous parabolic reflective surface in (a). According to the sampling theorem first formalized by Shannon [29, 30], the discrete current distribution $f_{S}(x)$ must sample the continuous distribution at an interval no greater than $\lambda / 2$ in order to reconstruct the original far-field radiation pattern.

According to the surface equivalence principle, first described by Christiaan Huygens and later formalized by Schelkunoff [31], any configuration of internal electric and magnetic sources enclosed in a region resulting in a current distribution at the surface of the region (e.g. the aperture plane) which is identical to $f_{S}(x)$ can be considered an equivalent system producing identical radiated electromagnetic fields outside of said region. One possible equivalent system is a phased array depicted in Figure 2.3.1(c), comprised of a plurality of closely-spaced radiators. 
In this case, the current distribution of the array $f_{a}(x)$ is identical to $f_{s}(x)$; therefore, the radiation patterns $\left|F_{a}(u)\right|$ and $\left|F_{s}(u)\right|$ are equivalent assuming Shannon's sampling criterion is satisfied. An undersampled aperture undergoes aliasing, resulting in grating lobes, analogous to overlapping spectral images of a discrete-time filter.

Similar to the parabolic reflector in (a) and the discretized reflector in (b), the array in (c) performs a coherent weighted sum to produce a far-field beam pattern. In this case, however, the amplitude weights and phase offsets are variable for each element in the array and are applied using beamforming electronics. The current distribution at the aperture plane is electronically configurable, and therefore the far-field radiation pattern, which represents its Fourier transform, is also configurable. Again, this is analogous to the capability of a digital filter's impulse response to reconfigure its temporal frequency response.

Electronic beam steering, as opposed to mechanical steering in the case of the parabolic reflector, allows for rapid reconfiguration of the beam pattern consistent with the property of a Fourier transform pair which maintains that a spatial shift in the $u v$-domain relates to a phase shift in the $x y$-domain. That is, assuming $F(u, v) \stackrel{\Im}{\Leftrightarrow} f(x, y)$, then $F\left(u-u_{0}, v-v_{0}\right)$ $\stackrel{\widetilde{J}}{\Leftrightarrow} f\left(x e^{j u_{0}}, y e^{j v_{0}}\right)$

Electronic beam steering is not mutually exclusive of mechanical steering. It may be the case that the individual elements in a phased array are mechanically-steered parabolic reflector antennas, the case for nearly all interferometric radiotelescopes [28]. In this case, the mechanical limitations of inertial movements do not undermine the requirements for beam agility. For instance, the very large array (VLA) in Socorro, New Mexico consists of 27 separate 25-meter parabolic reflectors, and the very large baseline array (VLBA) consists of 10 separate 25-meter parabolic reflectors positioned across the United States and 4 other radiotelescopes in other 
continents. The VLBA realizes an extremely sparse phased array capable of angular resolutions commensurate with an aperture size approaching the diameter of the Earth [32, 33].

Excluding applications involving long integration times, agile electronic beam scanning is generally the most salient and exploited attribute of the phased array antenna. Radar implementations typically rely on very agile surveillance and tracking beams, and mobile communications arrays rely on agility to compensate for motion. By scanning its beam in elevation, the very first operational phased array was able to overcome frequency selective fading caused by ionospheric reflections of a transatlantic short-wave radio link [34, 35, 36].

In addition to electronic beam steering, there are many other advantages of the phased array antenna. The control of sidelobes is a primary advantage in radar and communications applications. Sidelobes represent spatial regions in which the antenna radiates or receives unwanted and appreciable power, analogous to stopband ripple encountered in filter theory. In radar applications, sidelobes lead to spatial ambiguities and clutter [37]. In communications applications, sidelobes lead to increased antenna noise temperature, increased susceptibility to interference and jamming, and increased probability of intercept [28]. In radioastronomy applications, sidelobes lead to degraded image quality and are conventionally suppressed using techniques requiring much longer integration times than radar [32].

A commonly employed technique to control sidelobes known as classical amplitude tapering, analogous to the concept of windowing in digital filter theory, is illustrated in Figure 2.3.2(a). Recall, the uniform current distribution in Figure 2.3.1(c) produces a narrow beam pattern with relatively high sidelobes. In contrast, the tapered current distribution in Figure 2.3.2(a) produces a broadened beam with lower sidelobes.

A popular alternative method to control sidelobes is known as adaptive nulling $[38,39]$. This method is similar to the synthesis procedures in adaptive filter theory, wherein specific regions of the beam pattern are dynamically suppressed using the degrees of freedom offered by amplitude 
and phase control, oftentimes phase-only control [40,39], of the elements within the array. In the 1970s, Tsandoulas of MIT's Lincoln Laboratory demonstrated a phased array using classical amplitude tapering which achieved nearly $40 \mathrm{~dB}$ of average sidelobe suppression [41], and in the 1990s, his colleagues reported an array employing adaptive sidelobe suppression and highly controlled amplitude and phase errors which achieved $60 \mathrm{~dB}$ sidelobe suppression [42, 43]. As will be discussed in later chapters, both amplitude and phase error contribute to minimum achievable sidelobe level as excellently elucidated by Mallioux in [44].

It is worthy of mention that one disadvantage of an electronically-steered array antenna, in comparison with a mechanically-steered reflector antenna, is the variation of sidelobe structure as a function of scan angle. Nonetheless, the phased array offers a significant advantage in sidelobe restructuring, a feature that is more difficult to implement in fixed aperture antennas.

More generally, phased arrays afford the opportunity to synthesize arbitrary radiation patterns with simple signal processing operations, including the formation of multiple arbitrarily shaped beams or nulls, where each beam has a favored polarization or frequency band. Figure 2.3.2(c) illustrates two independent and simultaneous far-field beam patterns associated with two different sets of amplitude and phase distributions applied to individual elements in a single aperture. These two beam patterns may equally represent two independent beams of orthogonal or coincident polarization, two independent beams of opposite or shared direction, or two independent beams favoring the same or different frequency band. When drawn differently, these two beams might also represent a single beam comprised of two peaks.

The same beamforming operations that produce peaks can similarly generate nulls. Radars often utilize sum and difference beams for monopulse tracking of multiple targets as well as multiple beams and polarizations to improve synthetic aperture radar (SAR) and interferometric SAR (ISAR) performance [28]. Another invaluable feature is the ability for a radar to detect the angle of arrival of interfering signals and adaptively place nulls while performing its mission 
uninterrupted. Airborne radars often exploit the capability of the phased array to simultaneously generate both low and high resolution beams to simultaneously track targets and survey surrounding territory [28]. Satellite communication subsystems (namely, the tracking, telemetry, and control, or "TT\&C" subsystems) often utilize monopulse beams to generate pointing error signals serving as feedback to precision antenna pointing control systems of on-board high-gain antennas [45].



(a)

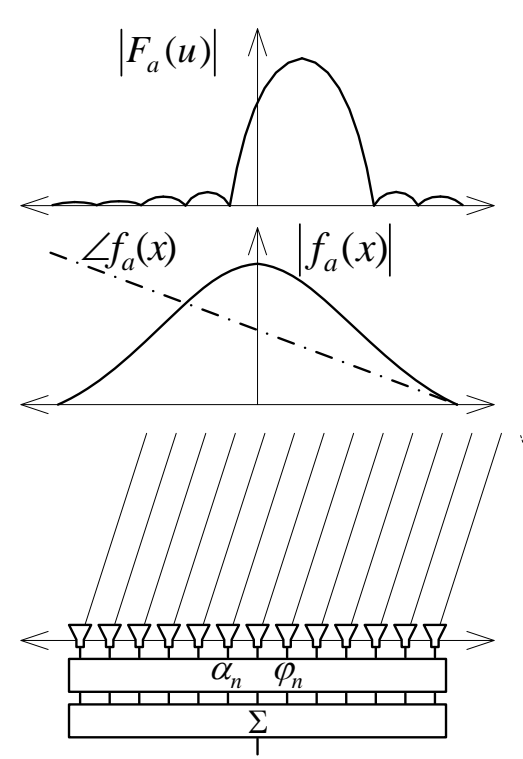

(b)

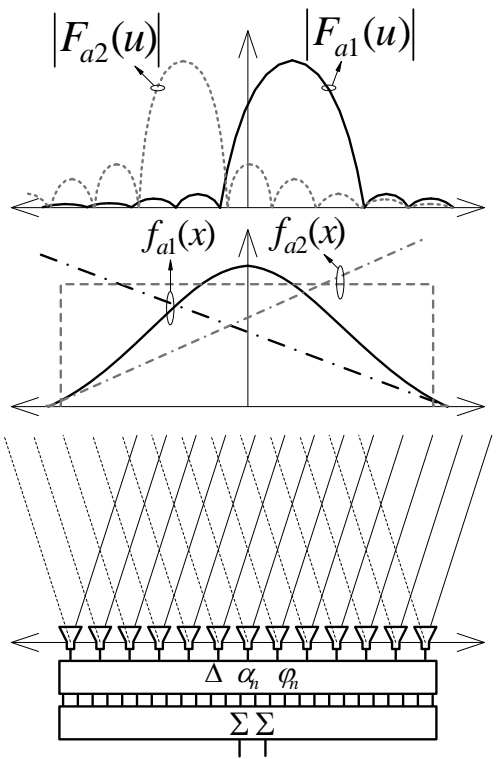

(c)

Figure 2.3.2 Three primary advantages of the phased array antenna: (a) sidelobe control, (b) beam scanning, and (c) arbitrary multi-beam shaping. 


\section{Chapter 3}

\section{Phased Array Theory}

\subsection{Electromagnetic Radiation}

\subsubsection{Maxwell's Equations}

In 1873, Scottish Physicist James C. Maxwell presented a renowned set of equations unifying the mathematics of electricity and magnetism. Beginning with the time-harmonic form of Maxwell's equations, which were expressed in the modern presentation by Oliver Heaviside, an expression for the electric field intensity of a radiating antenna element will be derived. The timeharmonic form of Maxwell's equations for a source charge density $\rho$ and corresponding source current density $\vec{J}$ are given by

$$
\begin{gathered}
\nabla \times \vec{E}=-j \omega \mu \vec{H} \\
\nabla \times \vec{H}=-j \omega \varepsilon \vec{E}+\vec{J} \\
\nabla \cdot \vec{E}=\frac{\rho}{\varepsilon} \\
\nabla \cdot \vec{H}=0 .
\end{gathered}
$$

where $\vec{E}$ represents the electric field intensity in units of volts per meter and $\vec{H}$ represents the magnetic field intensity in units of henries per meter. Maxwell's inclusion of the displacement 
current term in the second equation, known as Ampere's Law, leads to a central and profound conclusion: electromagnetic fields spread as polarized travelling waves at the speed of light.

Noting that the divergence of $\vec{H}$ is zero as expressed in the fourth equation of (3.1), $\vec{H}$ can be represented as a curl of an arbitrary vector potential term $\vec{A}$, where

$$
\vec{H}=\frac{1}{\mu} \nabla \times \vec{A}
$$

The relation in (3.2) relies on the vector identity requiring the divergence of the curl to be zero [46] for any vector; i.e., $\nabla \cdot \nabla \times \vec{\xi} \equiv 0$ for any $\vec{\xi}$. This vector potential represents a fictitious mathematical artifact that facilitates computation of real and physically meaningful properties such as currents and fields. The term $\vec{A}$ necessarily has units of henry-amps per meter and therefore represents a magnetic vector potential.

Substituting (3.2) into the first equation in (3.1) yields

$$
\nabla \times \vec{E}=-j \omega \mu\left(\frac{1}{\mu} \nabla \times \vec{A}\right)
$$

which simplifies to

$$
\nabla \times(\vec{E}+j \omega \vec{A})=0
$$

Defining the gradient of a second arbitrary scalar potential term $\Phi$ to represent the expression in parentheses employs the vector identity requiring the curl of a vector gradient to be zero; i.e., $\nabla \times \nabla \xi \equiv 0$ for any $\xi$. The scalar potential $\Phi$ has units of volts and therefore represents an electric scalar potential. After applying the identity and rearranging, a second substitution is given by

$$
\vec{E}=-j \omega \vec{A}-\nabla \Phi .
$$


The relationships in (3.2) and (3.5) express electric and magnetic field intensities, $\vec{E}$ and $\vec{H}$, in terms of the introduced vector and scalar potentials $\vec{A}$ and $\Phi$. Combining these relationships into the second equation in (3.1) and employing the vector identity, $\nabla \times \nabla \times \vec{\xi} \equiv \nabla(\nabla \cdot \vec{\xi})-\nabla^{2} \vec{\xi}$, yields

$$
\nabla^{2} \vec{A}+\omega^{2} \mu \varepsilon \vec{A}-\nabla(j \omega \mu \varepsilon \Phi+\nabla \cdot \vec{A})=-\mu \vec{J}
$$

Notice the expression in (3.6) contains both $\vec{A}$ and $\Phi$. As proven by Panofsky and Phillips, a vector in three dimensions is uniquely defined when the curl and divergence are defined as functions of the coordinates at all points in space, and if the totality of sources, as well as source density, is zero at infinity [47]. The equation in (3.2) defines the curl of $\vec{A}$. The next task is to completely describe $\vec{A}$ by defining its divergence, prompted by its appearance in (3.6). One definition that simplifies (3.6) by eliminating the third term is given by

$$
\nabla \cdot \vec{A}=j \omega \mu \varepsilon \Phi
$$

The expression in (3.7) is known as the Lorenz gauge condition, named after Danish Mathematician Ludvig Lorenz, and leads to the decoupling of variables in (3.6). Consequently, combining (3.6) and (3.7) yields the well-known vector wave equation

$$
\nabla^{2} \vec{A}+\omega^{2} \mu \varepsilon \vec{A}=-\mu \vec{J}
$$

The solution to the vector wave equation in (3.8) is given by

$$
\vec{A}=\iiint_{v^{\prime}} \mu \vec{J} \frac{e^{-j k \vec{r}}}{4 \pi \vec{r}} d v^{\prime}
$$


where $k=\omega \sqrt{\mu \epsilon}$ represents the wavenumber in units of radians per meter of a travelling wave propagating away from an excitation source contained within volume $v^{\prime}$ at a wave speed given by $v_{S}=\frac{1}{\sqrt{\mu \epsilon}}$. The coordinate system and geometry is illustrated in Figure 3.1.1.

Once $\vec{A}$ is determined, the magnetic field is computed using (3.2), and the electric field is computed by combining expressions in (3.5) and (3.7). Summarizing, the electric and magnetic fields are computed using the following relationships

$$
\begin{gathered}
\vec{H}=\frac{1}{\mu} \nabla \times \vec{A} \\
\vec{E}=-j \omega \vec{A}-\frac{\nabla(\nabla \cdot \vec{A})}{j \omega \mu \varepsilon} .
\end{gathered}
$$

In a source-free region where $\vec{J}=0$, e.g. the far-field region, the electric and magnetic fields are more easily computed using the following relationships

$$
\begin{gathered}
\vec{H}=\frac{1}{\mu} \nabla \times \vec{A} \\
\vec{E}=\frac{1}{j \omega \varepsilon} \nabla \times \vec{H} .
\end{gathered}
$$

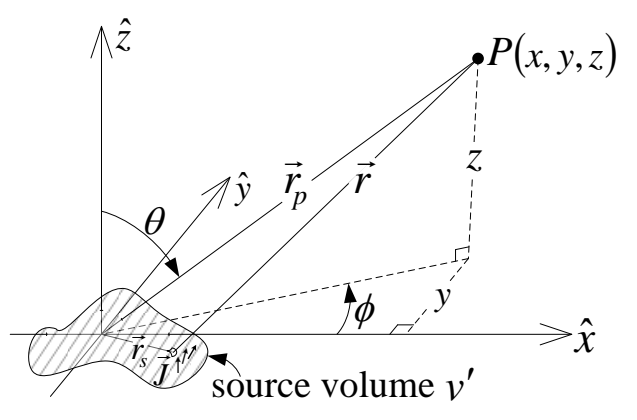

Figure 3.1.1 Geometry employed to compute the magnetic vector potential $\vec{A}$ and corresponding electric and magnetic fields $\vec{E}$ and $\vec{H}$ at a given point $\mathrm{P}$ resulting from a source current distribution $\vec{J}$ impressed upon source volume $v^{\prime}$ evaluated at distance vectors $\vec{r}=\vec{r}_{p}-\vec{r}_{s}$. 


\subsubsection{Power and the Poynting Vector}

An expression for complex power density known as the Poynting vector which has units of watts per square meter is given by

$$
\vec{S}=\frac{1}{2} \vec{E} \times \vec{H}^{*}
$$

The Poynting vector is sometimes useful for visualizing power flow in a circuit as well as power radiated from an antenna structure. The complex power flowing through any surface is

computed by integrating $\vec{S}$ over the surface. When $\vec{E}$ and $\vec{H}$ are in-phase, the Poynting vector is real-valued and radially-oriented, indicating the power is radiated; otherwise, power is considered reactive and complex. The real power flowing through surface $s$ is given by

$$
P=\operatorname{Re}\left\{\iint_{S} \vec{S} \cdot d \vec{S}\right\}=\frac{1}{2} \operatorname{Re}\left\{\iint_{S} \vec{E} \times \vec{H}^{*} \cdot d s \hat{n}\right\}
$$

where $\hat{n}$ is a unit vector normal to the surface $s$.

\subsection{Radiating Element}

\subsubsection{Hertzian Dipole}

The Hertzian dipole represents a problem with an important history. It is this very problem that was first investigated by Hertz [17]. Many electromagnetics textbooks [31, 48, 46, 16, 49] present the analysis of the infinitesimal or Hertzian electric dipole exclusively for the z-aligned current source. As an exercise and to maintain consistency with subsequent analyses for arrays oriented in the x-y plane, this analysis will be presented for a y-aligned current source as depicted in Figure 3.2.1. 


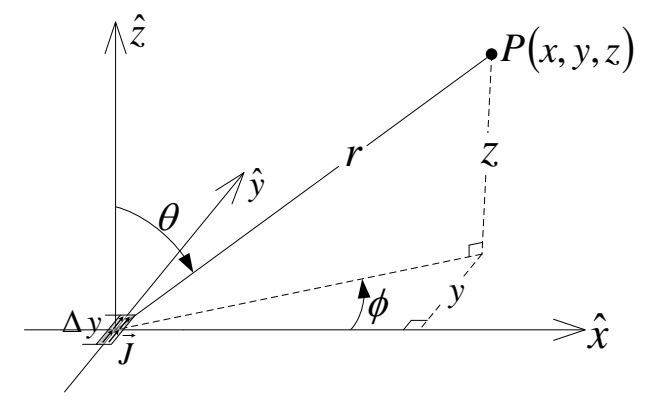

Figure 3.2.1 Geometry employed to compute the magnetic vector potential $\vec{A}$ and corresponding electric and magnetic fields $\vec{E}$ and $\vec{H}$ at a given point $\mathrm{P}$ resulting from a source current distribution $\vec{J}$ associated with a y-directed current source of infinitesimal length.

Beginning with the solution to the vector wave equation in (3.9), the magnetic vector potential of current distribution $\vec{J}=I \delta\left(x^{\prime}\right) \delta\left(z^{\prime}\right) \hat{y}$ in Figure 3.2.1 is given by

$$
\vec{A}=\iiint_{v^{\prime}} \mu \vec{J} \frac{e^{-j k \vec{r}}}{4 \pi \vec{r}} d v^{\prime}=\int_{-\infty}^{+\infty} \delta\left(x^{\prime}\right) d x^{\prime} \int_{-\infty}^{+\infty} \delta\left(z^{\prime}\right) d z^{\prime} \int_{-\Delta y / 2}^{\Delta y / 2} \mu I \hat{y} \frac{e^{-j k r}}{4 \pi r} d y^{\prime}
$$

where the radius $r$ has been substituted into the denominator and exponential, an uncommon substitution for the exponential term, but approximately true for an infinitesimal uniform current source. Computing the integral results in a single y-directed magnetic vector potential term

$$
\vec{A}=\frac{\mu I \Delta y e^{-j k r}}{4 \pi r} \hat{y} .
$$

The magnetic field is computed using the first equation in (3.11),

$$
\vec{H}=\frac{1}{\mu} \nabla \times \vec{A}=\frac{1}{\mu} \nabla \times\left(A_{y} \hat{y}\right)
$$

Rearranging according to the vector identity [16] given by $\nabla \times(\psi \vec{\xi})=(\nabla \psi) \times \vec{\xi}+$ $\psi(\nabla \times \vec{\xi})$ and noting that the second term vanishes when $\vec{\xi}$ is a constant vector yields 


$$
\vec{H}=\frac{1}{\mu}\left(\nabla A_{y}\right) \times \widehat{y}
$$

Computing the gradient given by $\nabla \psi=\frac{\partial \psi}{\partial r} \hat{r}+\frac{\partial \psi}{\partial \theta} \hat{\theta}+\frac{\partial \psi}{\partial \phi} \hat{\phi}$ yields

$$
\vec{H}=\frac{1}{\mu} \frac{\partial}{\partial r}\left(\frac{\mu I \Delta y e^{-j k r}}{4 \pi r}\right) \times \hat{y}=\frac{I \Delta y}{4 \pi}\left(\frac{-j k e^{-j k r}}{r}-\frac{e^{-j k r}}{r^{2}}\right) \hat{r} \times \hat{y}
$$

where the cross product of the unit vectors is given by

$$
\hat{r} \times \hat{y}=\cos \phi \hat{\theta}+\cos \theta \sin \phi \hat{\phi} .
$$

Therefore, the magnetic field is given by

$$
\vec{H}=\underbrace{\frac{I \Delta y k}{j 4 \pi}\left(1-\frac{1}{j k r}\right) \frac{e^{-j k r}}{r} \cos \phi}_{H_{\theta}} \hat{\theta}+\underbrace{\frac{I \Delta y k}{j 4 \pi}\left(1-\frac{1}{j k r}\right) \frac{e^{-j k r}}{r} \cos \theta \sin \phi}_{H_{\phi}} \hat{\phi} .
$$

Next, the electric field is computed using the second equation in (3.11),

$$
\begin{aligned}
\vec{E}=\frac{1}{j \omega \varepsilon} \nabla \times \vec{H} & =\frac{1}{j \omega \varepsilon r \sin \theta}\left[\frac{\partial}{\partial \theta}\left(H_{\phi} \sin \theta\right)-\frac{\partial H_{\theta}}{\partial \phi}\right] \hat{r} \\
& +\frac{1}{j \omega \varepsilon r}\left[\frac{1}{\sin \theta} \frac{\partial H_{r}}{\partial \theta}-\frac{\partial}{\partial r}\left(r H_{\phi}\right)\right] \hat{\theta} \\
& +\frac{1}{j \omega \varepsilon r}\left[\frac{\partial}{\partial r}\left(r H_{\theta}\right)-\frac{\partial H_{r}}{\partial \theta}\right] \hat{\phi} .
\end{aligned}
$$

Computing the relevant terms in the expression for $\vec{E}$ and simplifying yields

$$
\begin{aligned}
\vec{E}=\frac{I \Delta y \omega \eta}{2 \pi}\left(\frac{1}{j k r^{2}}-\frac{1}{r}\right) \frac{e^{-j k r}}{r} \frac{\sin \phi \cos ^{2} \theta}{\sin \theta} \hat{r} & \\
& +\frac{I \Delta y \omega \mu}{j 4 \pi}\left(1-\frac{1}{j k r}+\frac{1}{(k r)^{2}}\right) \frac{e^{-j k r}}{r} \cos \theta \sin \phi \hat{\theta} \\
& +\frac{I \Delta y \omega \mu}{j 4 \pi}\left(1-\frac{1}{j k r}+\frac{1}{(k r)^{2}}\right) \frac{e^{-j k r}}{r} \cos \phi \hat{\phi} .
\end{aligned}
$$


The expressions in (3.20) and (3.22) represent the magnetic and electric fields for a y-directed infinitesimal current source and are valid at any distance from the source. Constraining validity to the far-field region where $k r \gg 1$ leads to the following simplified expressions for the radiated fields:

$$
\begin{aligned}
\vec{H} & =\frac{I \Delta y k}{j 4 \pi} \frac{e^{-j k r}}{r}\{\cos \phi \hat{\theta}+\cos \theta \sin \phi \hat{\phi}\} \\
\vec{E} & =\frac{I \Delta y \omega \mu}{j 4 \pi} \frac{e^{-j k r}}{r}\{\cos \theta \sin \phi \hat{\theta}+\cos \phi \hat{\phi}\}
\end{aligned}
$$

As expected, current sources aligned with the $\mathrm{x}$ - and $\mathrm{z}$ - axes produce electric and magnetic fields similar in structure to (3.23), but corrected for the appropriate scalar projections of the $\hat{x}$ and $\hat{y}$ unit vectors onto the $\hat{\theta}$ and $\hat{\phi}$ unit vectors in the far-field. Specifically, for $\mathrm{x}$ - and z-aligned current sources, the fields in the far-field region are given by (3.24) and (3.25), respectively.

$$
\begin{gathered}
\vec{H}=\frac{j I \Delta y k}{4 \pi} \frac{e^{-j k r}}{r}\{\cos \theta \cos \phi \hat{\phi}-\sin \phi \hat{\theta}\} \\
\vec{E}=\frac{j I \Delta y \omega \mu}{4 \pi} \frac{e^{-j k r}}{r}\{\cos \theta \cos \phi \hat{\theta}-\sin \phi \hat{\phi}\} \\
\vec{H}=\frac{j I \Delta y k}{4 \pi} \frac{e^{-j k r}}{r} \sin \theta \hat{\phi} \\
\vec{E}=\frac{j I \Delta y \omega \mu}{4 \pi} \frac{e^{-j k r}}{r} \sin \theta \hat{\theta}
\end{gathered}
$$

Inspecting (3.23), (3.24), and (3.25) reveals the likely cause prompting textbook authors to work exclusively with z-aligned current sources. The scalar projection $\hat{z} \cdot \hat{\phi}=0$; as a result, the electric field depends only on $\theta$ and magnetic field depends only on $\phi$ in the far-field region. This fact may seem obvious and often goes without statement, but may not appreciated if not stated explicitly. 


\subsubsection{Electric Field Lines}

In his 1893 paper [17], Hertz presents the theory of the infinitesimal dipole and discusses propagation in air and along conductive wires. In doing so, he graphically illustrates the spatial orientation of electric field lines, referred to as lines of force, for consecutive instances in time to provide the reader with some degree of visual confirmation. This line of reasoning was found to be particularly didactic and is therefore included in this section. It is worthy of mention that Sophocles Orfinidis of Rutgers University has also presented a mathematically elegant rendition of Hertz's work and has made this information publically available at no cost.

The electric field in (3.22) diminishes as the inverse cube of the distance $r$ very near the source, and as the inverse square of $r$ further from the source, and as the inverse of $r$ at great distances from the source. Although this understanding is instructive, the goal of this next task is to derive and visualize the electric field lines of a Hertzian dipole near the source.

Recall, the time-harmonic expression for the electric field of a z-directed source of length $\Delta z$ is given by

$$
\begin{aligned}
\vec{E}=\frac{I \Delta z \omega \eta}{2 \pi}\left(\frac{1}{r}\right. & \left.+\frac{1}{j k r^{2}}\right) \frac{e^{-j k r}}{r} \cos \theta \hat{r} \\
& +\frac{j I \Delta z \omega \mu}{4 \pi}\left(1+\frac{1}{j k r}-\frac{1}{(k r)^{2}}\right) \frac{e^{-j k r}}{r} \sin \theta \widehat{\theta} .
\end{aligned}
$$

Restoring the $e^{+j \omega t}$ term in the time-harmonic expression in (3.26) yields

$$
\begin{aligned}
\vec{E}(r, t)= & \underbrace{\frac{I \Delta z \omega \eta}{2 \pi}\left(\frac{1}{r}+\frac{1}{j k r^{2}}\right) \frac{e^{+j \omega t} e^{-j k r}}{r} \cos \theta}_{E_{r}} \hat{r} \\
& +\underbrace{\frac{j I \Delta z \omega \mu}{4 \pi}\left(1+\frac{1}{j k r}-\frac{1}{(k r)^{2}}\right) \frac{e^{+j \omega t} e^{-j k r}}{r} \sin \theta}_{E_{\theta}} \hat{\theta} .
\end{aligned}
$$

Taking the real part of the complex expression, replacing $\eta$ with $\frac{k}{\omega \varepsilon}$, and rearranging yields 


$$
\begin{gathered}
E_{r}=I \Delta z\left[k \sin (k r-\omega t)+\frac{\cos (k r-\omega t)}{r}\right] \frac{2 \cos \theta}{4 \pi \varepsilon r^{2}} \\
E_{\theta}=I \Delta z\left[k \sin (k r-\omega t)+\frac{\cos (k r-\omega t)}{r}\right] \frac{2 \cos \theta}{4 \pi \varepsilon r^{2}} \\
-I \Delta z k^{2} \cos (k r-\omega t) \frac{\sin \theta}{4 \pi \varepsilon r}
\end{gathered}
$$

To simplify this expression, the following substitutions are proposed:

$$
\begin{gathered}
\xi=k r \\
\psi=\omega t \\
E_{0}=\frac{I \Delta z k^{3}}{4 \pi \varepsilon r} \\
f(\xi)=\sin (\xi-\psi)+\frac{\cos (\xi-\psi)}{\xi} \\
\frac{d f(\xi)}{d \xi}=\cos (\xi-\psi)-\frac{\cos (\xi-\psi)}{\xi^{2}}-\frac{\sin (\xi-\psi)}{\xi} .
\end{gathered}
$$

Substituting the relationships in (3.29) into (3.28) yields

$$
\begin{aligned}
E_{r} & =E_{0} \frac{2 \cos \theta}{\xi^{2}} f(\xi) \\
E_{\theta} & =-E_{0} \frac{\sin \theta}{\xi} \dot{f}(\xi)
\end{aligned}
$$

where $\dot{f}(\xi)=\frac{d f(\xi)}{d \xi}$

Recognizing that the electric field is tangential to its field lines and therefore is also tangential to a small radial displacement $\overrightarrow{d r}=d r \hat{r}+r d \theta \hat{\theta}$ along the tangent to a line, the following cross product is valid:

$$
\overrightarrow{d r} \times \vec{E}=0
$$

Computing the cross product produces the $\hat{\phi}$-directed term given by

$$
\left\{d r E_{\theta}-r d \theta E_{r}\right\} \hat{\phi}=0 .
$$


For field lines oriented in the $x z$-plane, $\phi=0$; therefore (3.32) yields

$$
\frac{d r}{d \theta}=\frac{r E_{r}}{E_{\theta}}
$$

Likewise, expressing (3.32) in terms of the substitution $\xi=k r$ yields

$$
\frac{d \xi}{d \theta}=\frac{\xi E_{r}}{E_{\theta}}
$$

Expressing (3.34) in terms of (3.30) yields

$$
\frac{d \xi}{d \theta}=-2 \cot \theta \frac{f(\xi)}{\dot{f}(\xi)}
$$

Rearranging variables and integrating both sides yields

$$
\int \frac{\dot{f}(\xi)}{f(\xi)} d \xi=\int-2 \cot \theta d \theta
$$

Recognizing the left expression is the well-known integral given by

$$
\int \frac{\dot{f}(\xi)}{f(\xi)} d \xi=\ln |f(\xi)|+\text { constant }
$$

and the right expression is the well known integral given by

$$
-\int 2 \cot \theta d \theta=-\ln \left|\sin ^{2} \theta\right|+\text { constant }
$$

the expression in (3.36) and hence in (3.34) produces the final expression for electric field lines, given by

$$
f(\xi) \sin ^{2} \theta=\text { constant }
$$

Replacing the substitutions $f(\xi), \xi$, and $\psi$ with their equivalents in (3.29) gives the final expression for the electric field lines, or more precisely, contours of the electric field in the $x z$ plane at time $t$ and radius $r$ from the origin: 


$$
\left[\sin (k r-\omega t)+\frac{\cos (k r-\omega t)}{k r}\right] \sin ^{2} \theta=\text { constant }
$$

The relationship in (3.40) has been computed in MATLAB at a few time instances coinciding with the time instances Hertz selected to include in his paper published in 1893. Images from his publication and contour plots computed in MATLAB are illustrated in Figure 3.2.2.
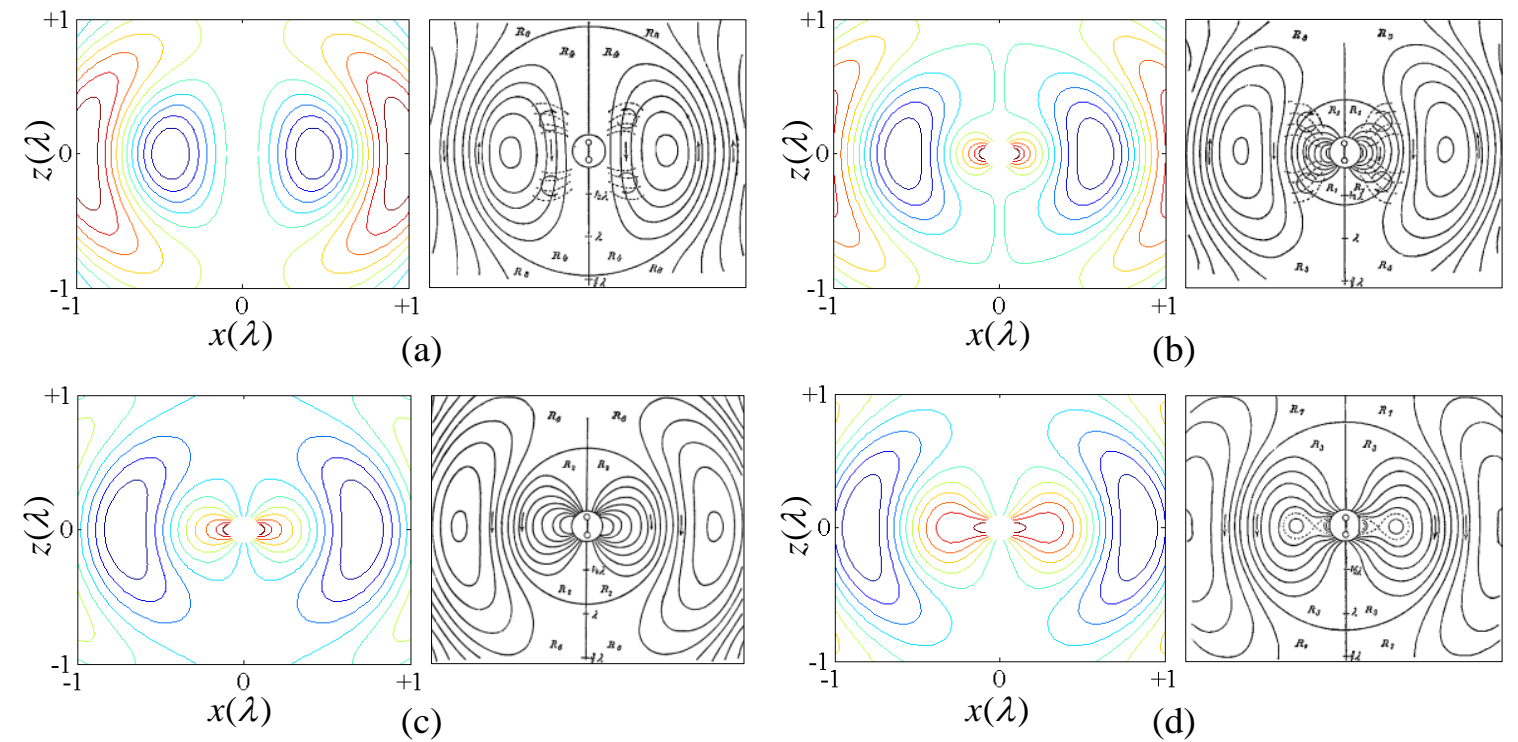

Figure 3.2.2 The electric field lines of a Hertzian dipole on the $x z$-plane at time $t_{0}+n T / 8$ where $T=2 \pi / \omega$ and (a) $n=0$, (b) $n=1$, (c) $n=2$, and (d) $n=3$. The left figures are contour plots generated in MATLAB, and the right figures are screenshots ${ }^{6}$ from Hertz's historical paper in 1893 [17].

\subsubsection{Active Element Pattern}

The antenna element pattern $\vec{e}(\theta, \phi)$ describes how an antenna radiates power as a function of spatial coordinates. The active element pattern refers to the antenna element pattern subjected to the array environment. In a closely-spaced array, radiating elements receive and re-radiate some of the power of neighboring elements. Additionally, neighboring elements cause near field

\footnotetext{
${ }^{6}$ The screenshots were obtained and processed using Snagit ${ }^{\circledR}$ screen capture software of the freely available digitization of Hertz's manuscript [8] provided by Google Books. Snagit ${ }^{\circledR}$ is a registered trademark of TechSmith Corporation. MATLAB is a registered trademark of The MathWorks, Inc.
} 
scattering and change the element pattern. It is the active element pattern that dictates the overall performance of the array.

\subsubsection{Directivity \& Gain}

The directivity of an antenna is computed directly from the antenna element pattern. Qualitatively, directivity reflects the degree to which an antenna concentrates radiated power relative to an isotropic radiator which projects power in all directions. A higher directivity indicates a higher concentration. Directivity is given by

$$
\vec{D}(\theta, \phi)=\frac{\sqrt{4 \pi} \vec{e}(\theta, \phi)}{\sqrt{\int_{0}^{2 \pi} \int_{0}^{\pi} \vec{e}(\theta, \phi) \sin \theta d \theta d \phi}} .
$$

Gain represents the directivity decreased by the total efficiency. Total efficiency includes dissipative losses, impedance mismatch losses, and radiation efficiency of the structure. Dissipative losses are related to the antenna's constitutive material properties.

\subsection{Array Fundamentals}

\subsubsection{Radiation Pattern}

For the one dimensional array of $N$ elements given in Figure 3.3.1, the array field $\vec{E}_{\text {array }}$ can be expressed as a superposition of the individual fields of each element in their respective array environments. Recall, the radiated electric field intensity for an element is given by

$$
\vec{E}(r, \theta, \phi)=A \frac{\exp \left(-j k_{0} r\right)}{r} \vec{e}(\theta, \phi)
$$

The individual fields generated by a given element are dependent on the properties of the excitation voltage, the natural radiation properties of the element structure, and the mutual coupling between the element and all other elements or objects in close proximity. 
In practice, a rigorous formulation of the total array electric field intensity requires the determination of the active element pattern for each element within the array. The active element pattern reflects the radiation properties of a radiating element when subject to the electromagnetic environment of the array. For clarity, the expression $\vec{e}(r, \theta, \phi)$ represents the active element pattern. This active element pattern is defined as the spatial distribution of field intensity of the radiating element as a function of spherical coordinates $(r, \theta, \phi)$. Unless otherwise specified, $\vec{e}(r, \theta, \phi)$ typically represents the field intensity in the far-field region where $r \gg \lambda_{0}$, and $\vec{e}(r, \theta, \phi)$ no longer depends on the distance from the source $r$.

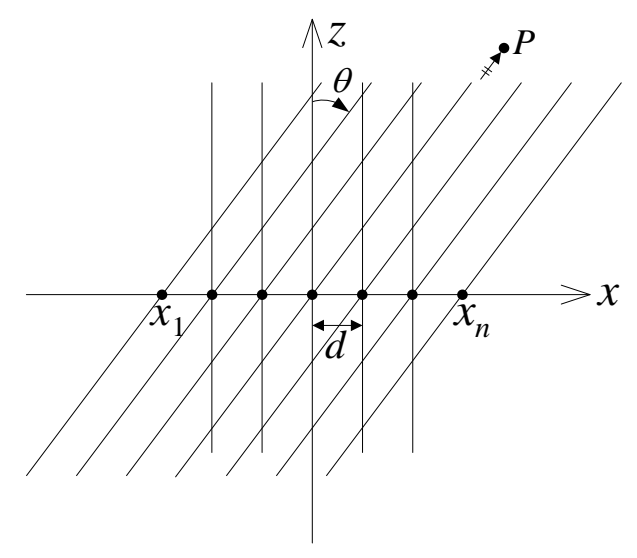

Figure 3.3.1 A one dimensional array described by lattice parameter $d$.

Invoking superposition, the array field intensity at an observation point in the far-field is given by

$$
\begin{aligned}
\vec{E}_{\text {array }}(\theta, \phi)= & \tilde{a}_{1} A \frac{\exp \left(-j k_{0} r_{1}\right)}{r_{1}} \vec{e}_{1}(\theta, \phi)+\tilde{a}_{2} A \frac{\exp \left(-j k_{0} r_{2}\right)}{r_{2}} \vec{e}_{2}(\theta, \phi) \\
& +\cdots+\tilde{a}_{n} A \frac{\exp \left(-j k_{0} r_{n}\right)}{r_{n}} \vec{e}_{n}(\theta, \phi)
\end{aligned}
$$

where $\tilde{a}_{n}$ represents a complex excitation coefficient applied to the $n$-th element, $k_{0}$ is the spatial frequency, and $r_{n}$ is the spatial distance from $P$ to the phase center of the $n$-th element. 
The expression in (3.43) represents an extensive computation prohibiting efficient design of practical arrays containing a large number of radiating elements. A more efficient computation assumes separable exponential terms and element field patterns. Three important assumptions afford this simplification, and they are easily derived with the geometry presented in Figure 3.3.2. This construction assumes the first element coincides with the origin and considers a second element at $x_{n}$. The first goal is determine the expression for $r_{n}$ in terms of $r_{1}$ and interelement spacing $a_{n}$. According to Figure 3.3.2,

$$
\begin{gathered}
r_{1}^{2}=x^{2}+y^{2}+z^{2} \\
r_{n}=\sqrt{\left(x-d_{n}\right)^{2}+y^{2}+z^{2}} \text { for } n=1,2, \ldots, N \\
x=r_{1} \sin \theta \cos \phi \\
z=r_{1} \cos \theta
\end{gathered}
$$

where $d_{n}=d(n-1)$ for $n=1,2, \ldots, N$. Combining the first two expressions in (3.44) and rewriting in the form of $(a+b)^{n}$ yields

$$
r_{n}=\left[r_{1}^{2}+\left(d_{n}^{2}-2 d_{n} x\right)\right]^{1 / 2}
$$


Applying the binomial theorem given by

$$
\begin{gathered}
(a+b)^{\xi}=\sum_{k=0}^{\xi}\left(\begin{array}{l}
\xi \\
k
\end{array}\right) a^{\xi-k} b^{k}=a^{\xi}+n a^{\xi-1} b+\frac{\xi(\xi-1)}{2 !} a^{\xi-2} b^{2} \\
+\frac{\xi(\xi-1)(\xi-2)}{3 !} a^{\xi-3} b^{3} \\
+\frac{\xi(\xi-1)(\xi-2)(\xi-3)}{4 !} a^{\xi-4} b^{4}+\cdots
\end{gathered}
$$

and performing algebraic manipulation yields

$$
r_{n}=r_{1}-d_{n} \sin \theta \cos \phi+\frac{d_{n}{ }^{2}}{r_{1}} \xi_{1}(\theta, \phi)+\frac{d_{n}{ }^{3}}{{r_{1}}^{2}} \xi_{2}(\theta, \phi)+\frac{d_{n}{ }^{4}}{r_{1}{ }^{3}} \xi_{3}(\theta, \phi)+\cdots
$$

where

$$
\begin{gathered}
\xi_{1}(\theta, \phi)=\frac{1}{2}\left[1-\sin ^{2} \theta \cos ^{2} \phi\right] \\
\xi_{2}(\theta, \phi)=\frac{1}{2} \sin \theta \cos \phi\left[1-\sin ^{2} \theta \cos ^{2} \phi\right] \\
\xi_{3}(\theta, \phi)=\frac{1}{8} \sin \theta \cos \phi\left[1-6 \sin ^{2} \theta \cos ^{2} \phi\right] \ldots
\end{gathered}
$$

for $n=1,2, \ldots, N$. The first assumption recognizes that the inverse of the spatial distance (i.e. $\left.1 / r_{n}\right)$ in (3.43) is a slow-varying function when $r_{1} \gg d_{n} \forall n$, a valid condition in the far-field region. According to (3.47), the second term is marginalized when $r_{1} \gg d_{n} \forall n$. Further, all subsequent terms are also marginalized when $r_{1}^{\xi} \gg d_{n}^{\xi+1} \forall n$. This implies that an appropriate approximation for $r_{n}$ is given by

$$
r_{n} \approx r_{1} \text { for } n=1,2, \ldots, N
$$


This approximation can be applied to the denominators in each term of (3.43).

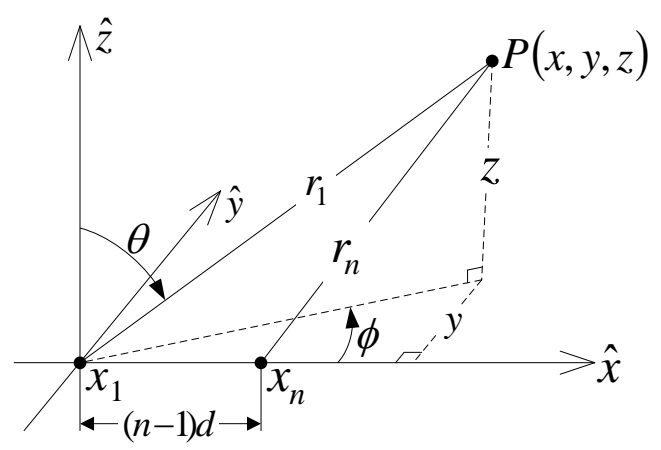

Figure 3.3.2 Coordinate geometry to facilitate the construction of assumptions employed to simplify the expression for array field intensity.

The approximation in (3.49), however, cannot be substituted into each instance of $r_{n}$ within the complex exponential terms in (3.43) because this term represents a highly oscillatory function, which leads to the second assumption. An approximation that retains the relative phase information associated with interelement spacing $a_{n}$ consists of the first two terms in (3.47) is given by

$$
r_{n} \approx r_{1}-d_{n} \sin \theta \cos \phi
$$

Again, all subsequent terms in (3.47) are marginalized when $r_{1}^{\xi} \gg d_{n}^{\xi+1} \forall n$ so this assumption is reasonable. Combining this result with $d_{n}=d(n-1)$ for $n=1,2, \ldots, N$ yields the final expression of the approximation used in the exponential terms of (3.43):

$$
r_{n} \approx r_{1}-(n-1) d \sin \theta \cos \phi \forall n
$$

To simply the analysis, the element patterns for all elements are assumed to be identical; that is,

$$
\vec{e}_{n}(\theta, \phi)=\vec{e}_{1}(\theta, \phi) \forall n
$$


Substituting the expressions for three assumptions - (3.49), (3.51), and (3.52) - into (3.43) yields the following array field intensity:

$$
\begin{aligned}
\vec{E}_{\text {array }}(\theta, \phi)= & A \vec{e}_{1}(\theta, \phi) \frac{\exp \left(-j k_{0} r_{1}\right)}{r_{1}}\left[\tilde{a}_{1}+\tilde{a}_{2} \exp \left(j k_{0} d \sin \theta \cos \phi\right)+\cdots\right. \\
& \left.+\tilde{a}_{n} \exp \left(j k_{0}(N-1) d \sin \theta \cos \phi\right)\right] \\
= & A \vec{e}_{1}(\theta, \phi) \frac{\exp \left(-j k_{0} r_{1}\right)}{r_{1}}\left[\sum_{n=1}^{N} \tilde{a}_{n} \exp \left(j k_{0}(n-1) d \sin \theta \cos \phi\right)\right]
\end{aligned}
$$

Applying the three approximations allows factorization of the element field intensity and radially dependent complex exponential term in accordance with the distributive property. The expression in the square brackets is of significant importance to the array architect; it represents the array factor $A F(\theta, \phi)$ which depends on the placement of radiating elements within the array and the complex weights or excitations provided to the radiating elements. A complex weight $\tilde{a}_{n}$ defines both the amplitude and phase of the excitation source, where $\tilde{a}_{n}=a e^{-j \alpha}$. These three design parameters — element placement, amplitude weights, and phase offsets-were the central concern of early phased array pioneers ${ }^{7}[50,51,52,53]$ and are discussed in the next section.

The middle complex exponential term $\psi=\exp \left(-j k_{0} r_{1}\right) / r_{1}$ represents a solution to the complex scalar wave or Helmholtz equation, $\nabla \psi+\beta^{2} \psi=0$, for an isotropic point source at a distance $r_{1}$ from the source. Ignoring this radial term and normalizing the element field intensity by its incident power yields

$$
\vec{E}_{\text {array }}(\theta, \phi)=\vec{G}_{1}(\theta, \phi)\left[\sum_{n=1}^{N} \tilde{a}_{n} \exp \left(j k_{0}(n-1) d \sin \theta \cos \phi\right)\right]
$$

\footnotetext{
${ }^{7}$ Although these pioneers quickly unearthed the underlying mathematics and exhaustively investigated the combinations associated with these three design parameters, half a century later, patents continue to be issued for specific combinations which exhibit some desirable attribute [70, 67].
} 
where $\vec{G}_{1}(\theta, \phi)$ is the element gain pattern and is normalized so that

$$
\int_{0}^{2 \pi} \int_{0}^{\pi}\left|\vec{G}_{1}(\theta, \phi)\right|^{2} \sin \theta d \theta d \phi=4 \pi(1-L)
$$

where L represents the antenna loss factor. Similarly, the gain of the array is the array field intensity pattern normalized by incident power of the array. The incident power of the array is given by

$$
P_{\text {in }}=\sum_{n=1}^{N}\left|\tilde{a}_{n}\right|^{2}
$$

so the normalized gain pattern or array radiation pattern is given by

$$
\vec{G}_{\text {array }}(\theta, \phi)=\frac{\vec{G}_{1}(\theta, \phi)}{\sum_{n=1}^{N}\left|\tilde{a}_{n}\right|^{2}}\left[\sum_{n=1}^{N} \tilde{a}_{n} \exp \left(j k_{0}(n-1) d \sin \theta \cos \phi\right)\right]
$$

The expression in (3.57) represents the three dimensional radiation pattern for a one dimensional linear array of elements with fixed interelement spacing. Extending this expression to a planar array of elements with arbitrary element location $\left(x_{n}, y_{n}\right) \forall n$, the array radiation pattern can be expressed as

$$
\begin{aligned}
\vec{G}_{\text {array }}(\theta, \phi)= & \frac{\vec{G}_{1}(\theta, \phi)}{\sum_{n=1}^{N}\left|\tilde{a}_{n}\right|^{2}}\left[\sum _ { n = 1 } ^ { N } \tilde { a } _ { n } \operatorname { e x p } \left(j k_{0} x_{n} \sin \theta \cos \phi\right.\right. \\
& \left.\left.+j k_{0} y_{n} \sin \theta \sin \phi\right)\right]
\end{aligned}
$$

where, for the periodic two dimensional lattice examples given in Figure 3.3.3, the nth element is located at the pth oblique column and qth row, 


$$
x_{n}=p d+\frac{q b}{\tan \gamma} \text { and } y_{n}=q b \text { for } n=1,2, \ldots, N .
$$

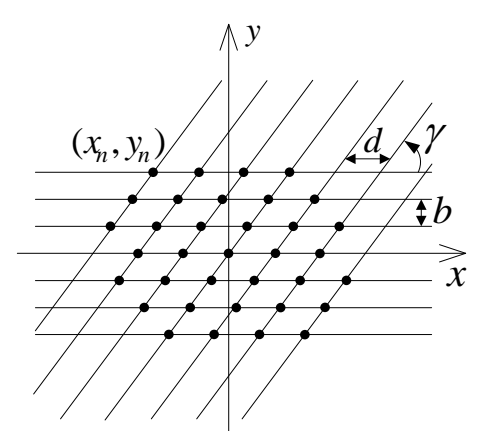

(a)

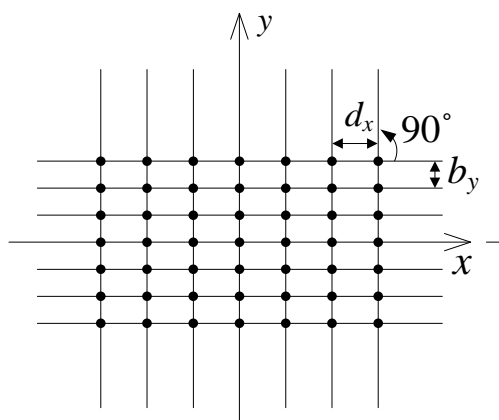

(b)

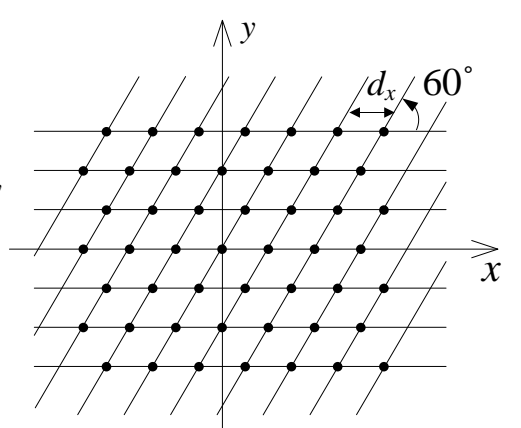

(c)

Figure 3.3 .3 (a) A general lattice of a two dimensional array is uniquely described by lattice parameters $[d, b, \gamma]$ and related to the location of each element $\left(x_{n}, y_{n}\right)$ by (3.59). Two common configurations are (b) the rectangular lattice described by $\left[d_{x}, b_{y}, 90^{\circ}\right]$ and (c) the equilateral triangular lattice described by $\left[d_{x}, 0.866 d_{x}, 60^{\circ}\right]$.

The array radiation pattern for a 256-element array oriented in an equilateral triangular lattice is illustrated in Figure 3.3.4. A lattice described by $\left[0.541 ", 0.469 ", 60^{\circ}\right]$ represents a unit cell that is $35 \%$ smaller than a dime. A plot of the computed 3D radiation pattern in decibels from (3.58) and (3.59) at $14.5 \mathrm{GHz}$ for the northern hemisphere illustrates the spatial selectivity contributed by the array factor within the square brackets of (3.58). That is, the array favors radiation along the boresight axis when $\tilde{a}_{n}=1 \forall n$ indicated by the main lobe, but as a result of the summation of complex exponentials in the array factor, also favors radiation in other directions, dependent on the array factor and lattice arrangement. These additional peaks are known as sidelobes and grating lobes. 


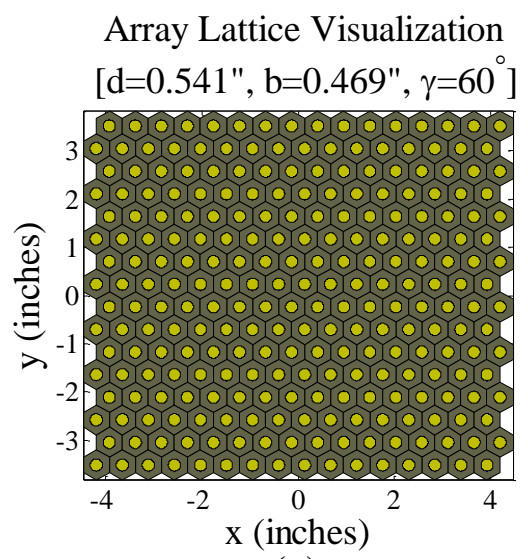

(a)

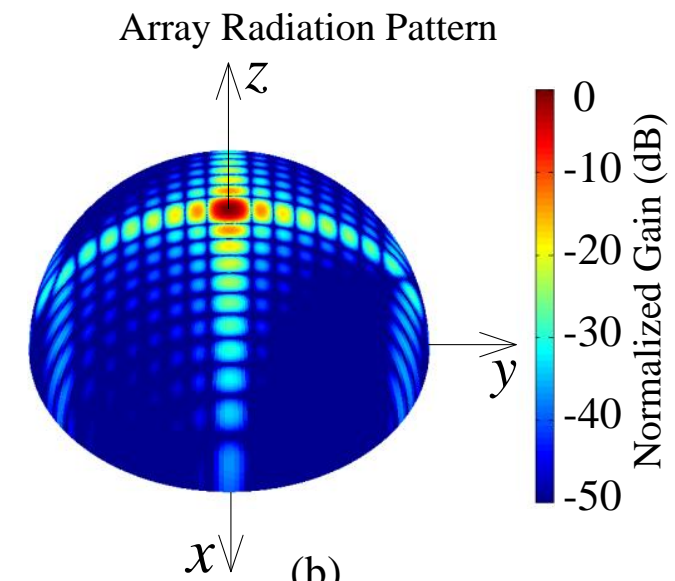

(b)

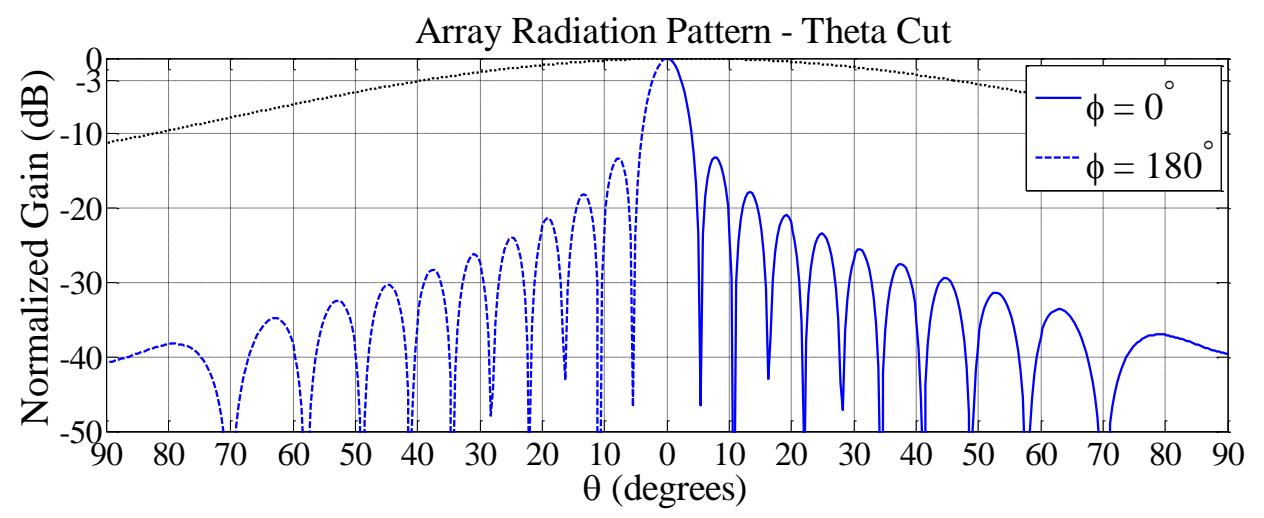

(c)

Figure 3.3.4 A $16 \times 16$ element array oriented in (a) an equilateral triangular lattice produces the $3 \mathrm{D}$ and $2 \mathrm{D}$ radiation patterns for $\tilde{a}_{n}=1 \forall n$ in (b) and (c), respectively, computed at $14.5 \mathrm{GHz}$.

As a summary to this discussion, the three assumptions enabling the application of the principle of pattern multiplication in Figure 3.3.4 are reiterated.

First, the expression in (3.49) equates the spatial distance from any observation point in the far-field to any of the elements within the array. This approximation applies to the $1 / r_{n}$ multiplicative factor in the expression for array field intensity. The error associated with this approximation is marginalized when the distance of the observer greatly exceeds the size of the array. As an illustration, consider a Ku-band phased array antenna for SATCOM on-the-move (SOTM) applications $[54,55]$. This application allows, for example, mobile broadband access to travelling passengers aboard commercial aircraft, high speed trains, or personal vehicles. A maximum array dimension of only one meter typically provides the required signal-to-noise ratio 
to ensure the communication link operates successfully, while the distance to the geosynchronous earth orbiting satellite is $35,786 \mathrm{~km}$ from earth sea-level (and roughly $35,776 \mathrm{~km}$ from commercial aircraft cruising altitude). Therefore, the error between distance from a center element to an edge element is infinitesimal and insignificant. This assumption is not always appropriate. Consider the actively researched applications of imaging or selectively ablating cancerous tumors within a human body. When employing a phased array in these applications, an accurate array field intensity computation may not allow the distance from the object to each of the elements in the array to be equated.

The second approximation truncates the summation in (3.47) to include the first two terms, leaving (3.51). Similarly, this assumption remains valid when the distance to the far-field observer greatly exceeds the maximum dimension of the array. In this case, phase errors are infinitesimal and do not meaningfully impact the field pattern when compared to other sources of phase error encountered in practical phased arrays, including phase and amplitude quantization errors and imprecision of practical electronic devices.

Lastly, all radiating elements are assumed to have identical radiation patterns consistent with an infinite array. Recall, the element pattern term in the expression for the array radiation pattern represents the element in its array environment, as opposed to an isolated environment, and also represents element pattern for all radiating elements in the array.

In general, it is not practical to implement an array with elements exhibiting identical radiation patterns; nonetheless, this approximation is reasonable under two conditions. The first condition considers the scan range of the array. Practical planar arrays are typically limited to $30^{\circ}$ and sometimes up to $60^{\circ}$ in conical scan capability. Beyond this limitation, it may be more advantageous to employ multiple planar facets covering desired spatial sectors [28]. Referring to the element pattern which is plotted in Figure 3.3.4(c), observe that gain varies moderately (i.e. less than $1.8 \mathrm{~dB}$ ) for $\theta<30^{\circ}$, and to a lesser extent for $\theta<60^{\circ}$. If edge elements in a finite array 
contribute asymmetrical gain patterns caused by the truncation of the ground plane or dielectrics at the edge of the array, the variation in gain is likely very small within the $30^{\circ}$ scan range and marginally affects the overall array pattern. Edge element asymmetry is illustrated for a linear array of microstrip patch antennas spaced $\sim \lambda / 2$ apart in [16].

There are two methods commonly employed to obtain the active element pattern. The first method $[27,16]$ is to empirically measure in an anechoic chamber a single element in a finite array with all other elements terminated with their respective line impedances $Z_{0}$ such that maximum power received at each terminated antenna is transferred to the load. This process extends to edge elements. If edge element patterns strongly differ from the central element, then an overall average may be computed which represents the active element pattern $\vec{G}_{1}(\theta, \phi)$ in the computation of array radiation pattern. An alternative method employs a 3D electromagnetic solver to simulate antenna elements subjected to boundary conditions of an infinite array or a truncated finite array, and again, an average element pattern can be computed. Some modern solvers are equipped with intuitive array modules facilitating this process [56].

\subsubsection{Directivity \& Beamwidth}

Similar to the definition for an individual element, the complex directive pattern of an array relies solely on the far-field electric intensity pattern of the array and is given by

$$
\vec{D}(\theta, \phi)=\frac{\sqrt{4 \pi} \vec{E}_{\text {array }}(\theta, \phi)}{\sqrt{\int_{0}^{2 \pi} \int_{0}^{\pi} \vec{E}_{\text {array }}(\theta, \phi) \sin \theta d \theta d \phi}}
$$

The directivity of the array $D(\theta, \phi)$ is defined as the square of the magnitude of $\vec{D}(\theta, \phi)$ and is expressed in units of $\mathrm{dBi}$ when normalized to an isotropic point source. When a single value is provided for directivity of an antenna or antenna array, it is assumed to represent the maximum directivity or the directivity at the intended look angle. 
The beamwidth of an array typically denotes the minimum beamwidth in the direction of mechanical boresight, normal to the aperture plane. Oftentimes, two values are specified to represent beamwidth in both cardinal planes. It is understood that the beamwidth of a beam scanned away from mechanical boresight will broaden by a factor of $1 / \cos \theta_{0}$, where $\theta_{0}$ represents the scan angle in elevation.

Oftentimes, system design tables approximate directivity or beamwidth when unknown or variable. A popular approach defines the maximum directivity for an aperture area $A$, given by

$$
D_{\max }=\frac{4 \pi A}{\lambda^{2}}
$$

and determines beamwidth based on a set of empirically determined constants for the directivity-beamwidth product. The directivity-beamwidth product was proposed and investigated by R. J. Stegen, C. Drane, and others in the 1960s [57, 58]. It allows for approximation of directivity or beamwidth when one parameter is known using the relationship given by

$$
D \approx \frac{D B}{B W_{E} B W_{H}}
$$

where $B W_{E}$ represents the e-plane beamwidth, and $B W_{H}$ represents the h-plane beamwidth. Useful directivity-beamwidth products are given in Table 3.3.1. Using the relationships in (3.61) and (3.62) along with Table 3.3.1, an array architect can reasonably approximate the size of an aperture and its corresponding beamwidth required for a given application assuming only the operational wavelength and desired directivity is known. If scan requirements are defined, the architect can also approximate the minimum number of antenna elements required to fill the aperture. 
Table 3.3.1 Directivity-beamwidth products for common aperture distributions.

\begin{tabular}{clc}
\hline Case & Aperture Distribution & DB $\left[\mathrm{deg}^{2}\right]$ \\
\hline 1 & No sidelobe pattern, rectangular beam & 41235 \\
2 & Uniform rectangular & 32383 \\
3 & Cosine-uniform rectangular & 35231 \\
4 & Gaussian & 36407 \\
5 & Uniform circular & 33709 \\
6 & Parabola-on-pedestal circular & 38933 \\
7 & General use for practical antennas & 26000 \\
\hline
\end{tabular}

\subsection{Array System Theory}

\subsubsection{Receive Operation \& G/T}

The ratio of antenna gain to system noise temperature, i.e. $\mathrm{G} / \mathrm{T}$, represents the primary figure or merit for the receive subsystem of a phased array antenna. G/T is used in radar and communications applications and is commonly expressed in units of $\mathrm{dB} / \mathrm{K}^{8}$. To compute $\mathrm{G} / \mathrm{T}$, one must develop a cascaded noise model [59]. This model allows one to compute the overall system noise temperature $(\mathrm{T})$ and facilitates sensitivity trades to assess how each parameter affects overall noise temperature. A model is typically constructed using a spreadsheet, MATLAB, or a circuit simulator (e.g. ADS budget simulator). A diagram for a cascaded model for a PCB-based $\mathrm{X} / \mathrm{Ku}$-band SATCOM array is given in Figure 3.4.1, and the computation for system noise temperature is given in Figure 3.4.2. Refer to the captions for the details of each model.

Sky temperature is an important aspect of array performance in receive mode. In the SHF band, atmospheric gases and weather-dependent phenomena are the largest contributors to absorption of microwave energy and resulting degradation of G/T. Atmospheric gases almost exclusively consist of nitrogen, argon, oxygen, carbon dioxide, and water vapor. Of these, oxygen and water vapor are largely responsible for absorption in the SHF band due to their dipole molecular structure. Atmosphere gases are modeled as a contiguous slab up to $50 \mathrm{~km}$ and hydrometeors up to $15 \mathrm{~km}$ [60]. Weather-dependent phenomena include clouds, fog, and rain.

\footnotetext{
${ }^{8}$ It should be noted that the logarithm is taken for both the numerator $(\mathrm{G})$ and denominator $(\mathrm{T})$ in this ratio, so the units are actually $\mathrm{dB} / \mathrm{dBK}$, or decibels per decibels-Kelvin.
} 
Other thermal noise radiators include extra-terrestrial and man-made noise. Extra-terrestrial noise is primarily sourced from the sun, moon, cosmic background, and the galaxy. The sun is the most prominent discrete noise source. Cosmic background noise serves as the lower bound for all sky temperature estimates at $2.7 \mathrm{~K}$ and is not a concern for satellite communications. Man-made noise includes emissions from electronics, power lines, and ignition systems. These sources become important when the main beam and sidelobes of an array are directed closer to the horizon. Accurate $\mathrm{G} / \mathrm{T}$ calculation requires proper estimation of these sources. Atmospheric models developed by the International Telecommunication Union (ITU) and Jet Propulsion Lab facilitate this process.

Once G/T has been computed for an array, it is utilized in a link calculation between the earth terminal and satellite to predict data rate for a given modulation format, bandwidth, and required signal-to-noise ratio. This link calculation requires the location of the satellite as well as its downlink EIRP. 


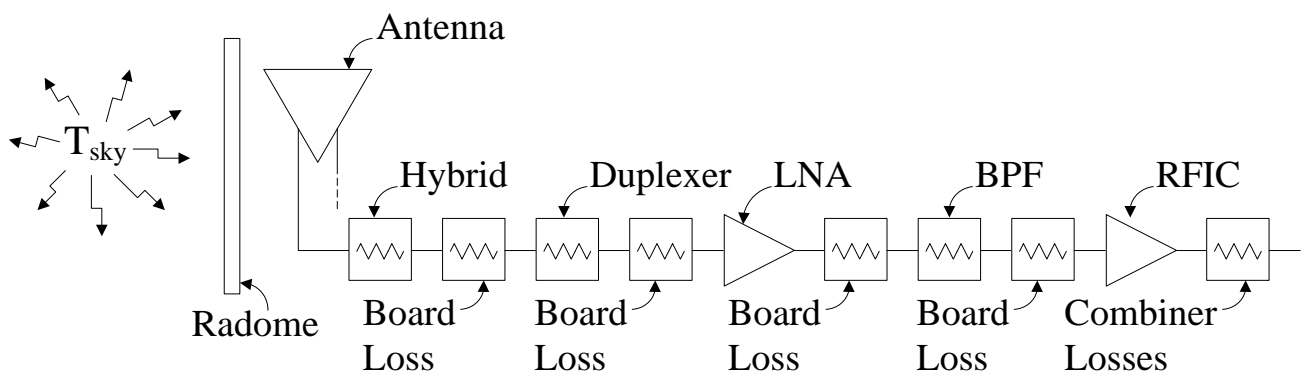

Figure 3.4.1 Cascaded receive chain used to compute system noise temperature and G/T of a PCB-based X/Ku-band SATCOM array. The sky temperature Tsky is a dynamic parameter that depends on many variables, including weather and other atmospheric conditions. For this application, atmospheric gases and weather-dependent phenomena primarily contribute to sky temperature $[60,61]$. Each element in the chain is represented by a gain and noise figure in Figure 3.4.2. The hybrid converts dual-linear to dual-circular polarization. The duplexer sets the noise bandwidth of the system and enables full-duplex operation since both $\mathrm{Rx}$ and Tx chains share the same antenna. The LNA is responsible for marginalizing downstream loss impacts to the system noise temperature. An RFIC is assumed to implement beamforming functionality (amplitude and phase offsets) as well as integrate combiners, memory, and a serial communication interface. The final block represents the losses encountered in the subsequent combiners and the down-conversion stage. Board loss elements represent transmission line and transition losses. 


\begin{tabular}{|c|c|c|c|c|c|c|c|c|c|c|c|c|}
\hline Frequency $=10.7 \mathrm{GHz}$ & Sky Temp. & Radome & Hybrid & Board Loss & Duplexer & Board Loss & LNA & Board Loss & Rx BPF & Board Loss & Rx RFIC & Comb. Loss \\
\hline Stage Gain & & $-0.25 \mathrm{~dB}$ & $-0.40 \mathrm{~dB}$ & $-0.75 \mathrm{~dB}$ & $-1.50 \mathrm{~dB}$ & $-0.30 \mathrm{~dB}$ & $24.00 \mathrm{~dB}$ & $-0.30 \mathrm{~dB}$ & $-2.20 \mathrm{~dB}$ & $-1.00 \mathrm{~dB}$ & $3.00 \mathrm{~dB}$ & $-6.00 \mathrm{~dB}$ \\
\hline Stage Noise Figure & & $0.25 \mathrm{~dB}$ & $0.40 \mathrm{~dB}$ & $0.75 \mathrm{~dB}$ & $1.50 \mathrm{~dB}$ & $0.30 \mathrm{~dB}$ & $1.50 \mathrm{~dB}$ & $0.30 \mathrm{~dB}$ & $2.20 \mathrm{~dB}$ & $1.00 \mathrm{~dB}$ & $9.00 \mathrm{~dB}$ & $6.00 \mathrm{~dB}$ \\
\hline Stage Noise Temperature & $15.0 \mathrm{~K}$ & $17.2 \mathrm{~K}$ & $28.0 \mathrm{~K}$ & $54.7 \mathrm{~K}$ & $119.6 \mathrm{~K}$ & $20.7 \mathrm{~K}$ & $119.6 \mathrm{~K}$ & $20.7 \mathrm{~K}$ & $191.3 \mathrm{~K}$ & $75.1 \mathrm{~K}$ & $2013.6 \mathrm{~K}$ & $864.5 \mathrm{~K}$ \\
\hline Stage Contribution to Tsys & $15.0 \mathrm{~K}$ & $17.2 \mathrm{~K}$ & $29.6 \mathrm{~K}$ & $63.5 \mathrm{~K}$ & $165.1 \mathrm{~K}$ & $40.4 \mathrm{~K}$ & $250.0 \mathrm{~K}$ & $0.2 \mathrm{~K}$ & $1.7 \mathrm{~K}$ & $1.1 \mathrm{~K}$ & $37.5 \mathrm{~K}$ & $8.1 \mathrm{~K}$ \\
\hline Tx Leakage Contribution to Tsys & & & & & & & $39.2 \mathrm{~K}$ & & & & & \\
\hline Cumulative System Noise Temp & $15.0 \mathrm{~K}$ & $32.2 \mathrm{~K}$ & $61.8 \mathrm{~K}$ & $125.3 \mathrm{~K}$ & $290.5 \mathrm{~K}$ & $330.9 \mathrm{~K}$ & $579.6 \mathrm{~K}$ & $579.8 \mathrm{~K}$ & $581.3 \mathrm{~K}$ & $582.4 \mathrm{~K}$ & $619.9 \mathrm{~K}$ & $628 \mathrm{~K}$ \\
\hline $\mathrm{G} / \mathrm{T}$ for $\mathrm{G}=0 \mathrm{dBic}$ & $-11.76 \mathrm{~dB} / \mathrm{K}$ & $-15.08 \mathrm{~dB} / \mathrm{K}$ & $-17.91 \mathrm{~dB} / \mathrm{K}$ & $-20.98 \mathrm{~dB} / \mathrm{K}$ & $-24.63 \mathrm{~dB} / \mathrm{K}$ & $-25.20 \mathrm{~dB} / \mathrm{K}$ & $-27.63 \mathrm{~dB} / \mathrm{K}$ & $-27.63 \mathrm{~dB} / \mathrm{K}$ & $-27.64 \mathrm{~dB} / \mathrm{K}$ & $-27.65 \mathrm{~dB} / \mathrm{K}$ & $-27.92 \mathrm{~dB} / \mathrm{K}$ & $-27.98 \mathrm{~dB} / \mathrm{K}$ \\
\hline Frequency $=11.7 \mathrm{GHz}$ & Sky Temp. & Radome & Hybrid & Board Loss & Duplexer & Board Loss & LNA & Board Loss & Rx BPF & Board Loss & Rx RFIC & Comb. Loss \\
\hline Stage Gain & & $-0.25 \mathrm{~dB}$ & $-0.40 \mathrm{~dB}$ & $-0.80 \mathrm{~dB}$ & $-1.50 \mathrm{~dB}$ & $-0.35 \mathrm{~dB}$ & $23.50 \mathrm{~dB}$ & $-0.35 \mathrm{~dB}$ & $-2.00 \mathrm{~dB}$ & $-1.10 \mathrm{~dB}$ & $3.00 \mathrm{~dB}$ & $-6.20 \mathrm{~dB}$ \\
\hline Stage Noise Figure & & $0.25 \mathrm{~dB}$ & $0.40 \mathrm{~dB}$ & $0.80 \mathrm{~dB}$ & $1.50 \mathrm{~dB}$ & $0.35 \mathrm{~dB}$ & $1.30 \mathrm{~dB}$ & $0.35 \mathrm{~dB}$ & $2.00 \mathrm{~dB}$ & $1.10 \mathrm{~dB}$ & $9.00 \mathrm{~dB}$ & $6.20 \mathrm{~dB}$ \\
\hline Stage Noise Temperature & $15.0 \mathrm{~K}$ & $17.2 \mathrm{~K}$ & $28.0 \mathrm{~K}$ & $58.7 \mathrm{~K}$ & $119.6 \mathrm{~K}$ & $24.3 \mathrm{~K}$ & $101.2 \mathrm{~K}$ & $24.3 \mathrm{~K}$ & $169.6 \mathrm{~K}$ & $83.6 \mathrm{~K}$ & $2013.6 \mathrm{~K}$ & $918.9 \mathrm{~K}$ \\
\hline Stage Contribution to Tsys & $15.0 \mathrm{~K}$ & $17.2 \mathrm{~K}$ & $29.6 \mathrm{~K}$ & $68.1 \mathrm{~K}$ & $167.1 \mathrm{~K}$ & $48.0 \mathrm{~K}$ & $216.4 \mathrm{~K}$ & $0.2 \mathrm{~K}$ & $1.8 \mathrm{~K}$ & $1.4 \mathrm{~K}$ & $42.6 \mathrm{~K}$ & $9.7 \mathrm{~K}$ \\
\hline Tx Leakage Contribution to Tsys & & & & & & & $40.1 \mathrm{~K}$ & & & & & \\
\hline Cumulative System Noise Temp & $15.0 \mathrm{~K}$ & $32.2 \mathrm{~K}$ & $61.8 \mathrm{~K}$ & $129.9 \mathrm{~K}$ & $297.0 \mathrm{~K}$ & $345.0 \mathrm{~K}$ & $553.5 \mathrm{~K}$ & $553.7 \mathrm{~K}$ & $555.2 \mathrm{~K}$ & $556.6 \mathrm{~K}$ & $599.2 \mathrm{~K}$ & $609 \mathrm{~K}$ \\
\hline $\mathrm{G} / \mathrm{T}$ for $\mathrm{G}=0 \mathrm{dBi}$ & $-11.76 \mathrm{~dB} / \mathrm{K}$ & $-15.08 \mathrm{~dB} / \mathrm{K}$ & $-17.91 \mathrm{~dB} / \mathrm{K}$ & $-21.14 \mathrm{~dB} / \mathrm{K}$ & $-24.73 \mathrm{~dB} / \mathrm{K}$ & $-25.38 \mathrm{~dB} / \mathrm{K}$ & $-27.43 \mathrm{~dB} / \mathrm{K}$ & $-27.43 \mathrm{~dB} / \mathrm{K}$ & $-27.44 \mathrm{~dB} / \mathrm{K}$ & $-27.46 \mathrm{~dB} / \mathrm{K}$ & $-27.78 \mathrm{~dB} / \mathrm{K}$ & $-27.85 \mathrm{~dB} / \mathrm{K}$ \\
\hline Frequency $=12.7 \mathrm{GHz}$ & Sky Temp. & Radome & Hybrid & Board Loss & Duplexer & Board Loss & LNA & Board Loss & Rx BPF & Board Loss & Rx RFIC & Comb. Loss \\
\hline Stage Gain & & $-0.25 \mathrm{~dB}$ & $-0.40 \mathrm{~dB}$ & $-0.85 \mathrm{~dB}$ & $-1.50 \mathrm{~dB}$ & $-0.40 \mathrm{~dB}$ & $23.00 \mathrm{~dB}$ & $-0.40 \mathrm{~dB}$ & $-2.20 \mathrm{~dB}$ & $-1.20 \mathrm{~dB}$ & $3.00 \mathrm{~dB}$ & $-6.40 \mathrm{~dB}$ \\
\hline Stage Noise Figure & & $0.25 \mathrm{~dB}$ & $0.40 \mathrm{~dB}$ & $0.85 \mathrm{~dB}$ & $1.50 \mathrm{~dB}$ & $0.40 \mathrm{~dB}$ & $1.50 \mathrm{~dB}$ & $0.40 \mathrm{~dB}$ & $2.20 \mathrm{~dB}$ & $1.20 \mathrm{~dB}$ & $9.00 \mathrm{~dB}$ & $6.40 \mathrm{~dB}$ \\
\hline Stage Noise Temperature & $15.0 \mathrm{~K}$ & $17.2 \mathrm{~K}$ & $28.0 \mathrm{~K}$ & $62.7 \mathrm{~K}$ & $119.6 \mathrm{~K}$ & $28.0 \mathrm{~K}$ & $119.6 \mathrm{~K}$ & $28.0 \mathrm{~K}$ & $191.3 \mathrm{~K}$ & $92.3 \mathrm{~K}$ & $2013.6 \mathrm{~K}$ & $975.9 \mathrm{~K}$ \\
\hline Stage Contribution to Tsys & $15.0 \mathrm{~K}$ & $17.2 \mathrm{~K}$ & $29.6 \mathrm{~K}$ & $72.8 \mathrm{~K}$ & $169.0 \mathrm{~K}$ & $55.8 \mathrm{~K}$ & $261.7 \mathrm{~K}$ & $0.3 \mathrm{~K}$ & $2.3 \mathrm{~K}$ & $1.8 \mathrm{~K}$ & $53.0 \mathrm{~K}$ & $12.9 \mathrm{~K}$ \\
\hline Tx Leakage Contribution to Tsys & & & & & & & $41.0 \mathrm{~K}$ & & & & & \\
\hline Cumulative System Noise Temp & $15.0 \mathrm{~K}$ & $32.2 \mathrm{~K}$ & $61.8 \mathrm{~K}$ & $134.6 \mathrm{~K}$ & $303.6 \mathrm{~K}$ & $359.5 \mathrm{~K}$ & $606.4 \mathrm{~K}$ & $606.7 \mathrm{~K}$ & $608.7 \mathrm{~K}$ & $610.5 \mathrm{~K}$ & $663.5 \mathrm{~K}$ & $676 \mathrm{~K}$ \\
\hline $\mathrm{G} / \mathrm{T}$ for $\mathrm{G}=0 \mathrm{dBic}$ & $-11.76 \mathrm{~dB} / \mathrm{K}$ & $-15.08 \mathrm{~dB} / \mathrm{K}$ & $-17.91 \mathrm{~dB} / \mathrm{K}$ & $-21.29 \mathrm{~dB} / \mathrm{K}$ & $-24.82 \mathrm{~dB} / \mathrm{K}$ & $-25.56 \mathrm{~dB} / \mathrm{K}$ & $-27.83 \mathrm{~dB} / \mathrm{K}$ & $-27.83 \mathrm{~dB} / \mathrm{K}$ & $-27.84 \mathrm{~dB} / \mathrm{K}$ & $-27.86 \mathrm{~dB} / \mathrm{K}$ & $-28.22 \mathrm{~dB} / \mathrm{K}$ & $-28.30 \mathrm{~dB} / \mathrm{K}$ \\
\hline Tx I & $\mathrm{K}$ & & & & & & & & & & & \\
\hline $\mathrm{Tx}$ & 54 & & & & & & & & & & & \\
\hline Diplexer Isolation & $42.00 \mathrm{~dB}$ & & & & & & & & & & & \\
\hline Tx Leakage & $18.8 \mathrm{~K}$ & & & & & & & & & & & \\
\hline
\end{tabular}

Figure 3.4.2 Cascaded system noise temperature calculated at 10.7, 11.7, and $12.7 \mathrm{GHz}$ for a $\mathrm{X} / \mathrm{Ku}$-band SATCOM array. The primary input variables to the table are highlighted yellow, and the computed results are highlighted blue. A sky noise temperature of $15 \mathrm{~K}$ assumes moderate humidity and heavy cloud conditions. Moderate humidity is defined as water vapor density of $5-20 \mathrm{~g} / \mathrm{m}^{3}$, and a heavy cloud condition is defined as liquid water content of $0.5 \mathrm{~g} / \mathrm{m}^{3}$ in the Slobin cloud model [60]. System noise temperature ranges between 609 to $676 \mathrm{~K}$ within the receive band. Filter losses are typical for thin film ceramic filters with the passband and stopband characteristics required. LNA gain and noise figure are typical of a $0.13-\mu \mathrm{m}$ GaAs HEMT device. An 8-channel 0.18- $\mu \mathrm{m}$ SiGe BiCMOS RFIC containing 6-bit phase shifters and 5-bit attenuators has been demonstrated with the gain and noise figure listed in the table [62]. Board losses assume realistic PCB trace lengths and losses. 


\begin{tabular}{|c|c|c|c|c|c|c|c|c|}
\hline \multirow{2}{*}{$\frac{\text { Parameter }}{\text { Tx Scan Range }}$} & \multirow{2}{*}{$\frac{\text { Value }}{45 \mathrm{deg}}$} & \multirow{2}{*}{$\begin{array}{l}\text { Parameter } \\
\text { Frequency }\end{array}$} & \multicolumn{3}{|c|}{ Receive Subsystem } & \multicolumn{3}{|c|}{ Transmit Subsystem } \\
\hline & & & $10.70 \mathrm{GHz}$ & $11.70 \mathrm{GHz}$ & $12.70 \mathrm{GHz}$ & $13.75 \mathrm{GHz}$ & $14.10 \mathrm{GHz}$ & $14.50 \mathrm{GHz}$ \\
\hline Tx Scan Range Buffer & $2.5 \mathrm{deg}$ & Wavelength & $2.80 \mathrm{~cm}$ & $2.56 \mathrm{~cm}$ & $2.36 \mathrm{~cm}$ & $2.18 \mathrm{~cm}$ & $2.13 \mathrm{~cm}$ & $2.07 \mathrm{~cm}$ \\
\hline Tx Grating Lobe Free Fmax & $14.50 \mathrm{GHz}$ & Aperture Area & 1.05 sq. $\mathrm{m}$ & 1.05 sq. $\mathrm{m}$ & 1.05 sq. $\mathrm{m}$ & 1.05 sq. $\mathrm{m}$ & 1.05 sq. $\mathrm{m}$ & 1.05 sq. $\mathrm{m}$ \\
\hline Tx Element Spacing, dX & $1.37 \mathrm{~cm}$ & Aperture Directivity, Max. & $42.2 \mathrm{dBi}$ & $43.0 \mathrm{dBi}$ & $43.7 \mathrm{dBi}$ & $44.4 \mathrm{dBi}$ & $44.6 \mathrm{dBi}$ & $44.9 \mathrm{dBi}$ \\
\hline Tx Element Spacing, dY & $1.19 \mathrm{~cm}$ & Total Number of Elements & 6400 & 6400 & 6400 & 6400 & 6400 & 6400 \\
\hline Tx Elements, $\mathrm{X}$ & 80 & Active Element Realized Gain & $4.8 \mathrm{dBci}$ & $5.3 \mathrm{dBci}$ & $6.0 \mathrm{dBci}$ & $6.0 \mathrm{dBic}$ & $6.2 \mathrm{dBic}$ & $6.4 \mathrm{dBic}$ \\
\hline Tx Elements, Y & 80 & Scan Loss at Scan Limit & $2.9 \mathrm{~dB}$ & $2.9 \mathrm{~dB}$ & $2.9 \mathrm{~dB}$ & $3.4 \mathrm{dBi}$ & $3.4 \mathrm{dBi}$ & $3.4 \mathrm{dBi}$ \\
\hline Tx Aperture Size, $X$ & 43.28 in & Sidelobe Control Loss & $1.9 \mathrm{~dB}$ & $1.9 \mathrm{~dB}$ & $1.9 \mathrm{~dB}$ & $1.9 \mathrm{dBi}$ & $1.9 \mathrm{dBi}$ & $1.9 \mathrm{dBi}$ \\
\hline Tx Aperture Size, Y & 37.48 in & Antenna Gain at Scan Limit & $38.1 \mathrm{dBci}$ & $38.6 \mathrm{dBci}$ & $39.3 \mathrm{dBci}$ & $38.8 \mathrm{dBic}$ & $39.0 \mathrm{dBic}$ & $39.2 \mathrm{dBic}$ \\
\hline Tx PA Output Power, Max & $14.5 \mathrm{dBm}$ & Approx. Boresight Beamwidth & $1.7 \mathrm{deg}$ & $1.6 \mathrm{deg}$ & $1.5 \mathrm{deg}$ & $1.3 \mathrm{deg}$ & $1.3 \mathrm{deg}$ & $1.3 \mathrm{deg}$ \\
\hline Tx Post-PA Loss, Avg & $3.5 \mathrm{~dB}$ & Approx. Beamwidth at Scan Limit & $3.3 \mathrm{deg}$ & $3.0 \mathrm{deg}$ & $2.8 \mathrm{deg}$ & $2.6 \mathrm{deg}$ & $2.5 \mathrm{deg}$ & $2.4 \mathrm{deg}$ \\
\hline Tx EIRP Required & $56.0 \mathrm{dBW}$ & System Noise Temperature & $628 \mathrm{~K}$ & $609 \mathrm{~K}$ & $676 \mathrm{~K}$ & - & - & - \\
\hline Rx Scan Range & $45 \mathrm{deg}$ & G/T at Boresight (Max) & $13.0 \mathrm{~dB} / \mathrm{K}$ & $13.6 \mathrm{~dB} / \mathrm{K}$ & $13.9 \mathrm{~dB} / \mathrm{K}$ & - & - & - \\
\hline Rx Scan Range Buffer & $2.5 \mathrm{deg}$ & G/T at Scan Limit (Min) & $10.1 \mathrm{~dB} / \mathrm{K}$ & $10.7 \mathrm{~dB} / \mathrm{K}$ & $11.0 \mathrm{~dB} / \mathrm{K}$ & - & - & - \\
\hline Rx Grating Lobe Free Fmax & $14.50 \mathrm{GHz}$ & G/T Required at Scan Limit & $9.0 \mathrm{~dB} / \mathrm{K}$ & $9.0 \mathrm{~dB} / \mathrm{K}$ & $9.0 \mathrm{~dB} / \mathrm{K}$ & - & - & - \\
\hline Rx Element Spacing, $\mathrm{dX}$ & $1.37 \mathrm{~cm}$ & G/T Margin at Scan Limit & $1.1 \mathrm{~dB}$ & $1.7 \mathrm{~dB}$ & $2.0 \mathrm{~dB}$ & - & - & - \\
\hline Rx Element Spacing, dY & $1.19 \mathrm{~cm}$ & Total Radiated Power & - & - & - & 81 Watts & 81 Watts & 81 Watts \\
\hline Rx Elements, $\mathrm{X}$ & 80 & EIRP at Boresight (Max) & - & - & - & & & \\
\hline Rx Elements, Y & 80 & EIRP at Scan Limit (Min) & - & - & - & $57.8 \mathrm{dBW}$ & $58.0 \mathrm{dBW}$ & $58.2 \mathrm{dBW}$ \\
\hline Rx Aperture Size, $X$ & 43.28 in & EIRP Required at Scan Limit & - & - & - & $56.0 \mathrm{dBW}$ & $56.0 \mathrm{dBW}$ & $56.0 \mathrm{dBW}$ \\
\hline Rx Aperture Size, Y & 37.48 in & EIRP Margin at Scan Limit & - & - & - & $1.8 \mathrm{~dB}$ & $2.0 \mathrm{~dB}$ & $2.2 \mathrm{~dB}$ \\
\hline Rx G/T Required & $9.0 \mathrm{~dB} / \mathrm{K}$ & & & & & & & \\
\hline
\end{tabular}

Figure 3.4.3 Computation of G/T and EIRP for a X/Ku-band SATCOM array application. Key input parameters are highlighted in yellow, and the results are highlighted in blue. In this example, the array consists of 6400 elements and achieves a G/T of $9 \mathrm{~dB} / \mathrm{K}$ and EIRP of $56 \mathrm{dBW}$ at a scan limit of 45 degrees with roughly 1-2 $\mathrm{dB}$ of margin. The given sidelobe control loss reflects a Taylor taper which provides $25 \mathrm{~dB}$ sidelobe suppression.

\subsubsection{Transmit Operation \& EIRP}

The effective isotropic radiated power (EIRP) is the primary figure of merit for the transmit subsystem of a phased array antenna. EIRP has units of $\mathrm{dBW}$ and represents the sum of transmitted power and antenna gain when both are expressed in decibels. An EIRP computation for a PCB-based X/Ku-band SATCOM array is illustrated in Figure 3.3.3.

Two important transmit subsystem parameters are beamwidth and maximum sidelobe level. For X/Ku-band SATCOM, the ITU limits the 3-dB beamwidth and sidelobe level to avoid interference with adjacent satellites that are spaced 2 degrees apart along the geosynchronous belt. Beamwidth is limited to 1.3 degrees, and sidelobe level is limited to $-25 \mathrm{~dB}$. In this application, the beamwidth drives aperture size, rather than limitations associated with power amplifiers (PAs). In the example given in Figure 3.3.3, the average output power level required at each PA is a modest $14.5 \mathrm{dBm}$. Highly efficient PAs with PAE between 50-60\% have been demonstrated a Ku-band. 


\section{Chapter 4}

\section{Design}

\subsection{Introduction}

The radiating element selection is an exciting and diverse trade to perform. Three primary performance parameters to consider are bandwidth, gain, and polarization. Other important factors are cost, yield, manufacturability, form factor, mass, scan impedance uniformity, gain pattern uniformity, materials and manufacturing process variation, power handling capability, and thermal and mechanical robustness.

\subsubsection{Performance}

Operational bandwidth flows directly from the application and serves as a reasonable starting point to select a topology. Bandwidth may consist of a single band or multiple bands. In a multiple band system, some applications may favor either single or multiple radiating element topologies. A single wideband antenna covering the entire operational bandwidth offers flexibility and efficient aperture utilization. Alternatively, multiple apertures comprised of narrower 
bandwidth radiating elements which collectively span the operational bandwidth simplifies radiating element and front-end filter designs. This important trade influences system-level design and occurs simultaneously with array analyses and aperture configuration trades.

Gain represents another important parameter to consider. In addition to peak gain, the architect must consider gain over the operational bandwidth, gain pattern shape, and efficiency. The candidate radiating element exhibiting the largest peak gain may not always represent the optimal selection. The architect must consider element pattern gain roll-off at the edges of the required scan volume as well as the uniformity within scan volume. Therefore, an array with a grating-lobe free scan range of 75 degrees may benefit from a radiating element with lower peak gain and correspondingly less gain roll-off at its scan limits, whereas an array with a scan range limited to less than 20 degrees may benefit most from an element with largest peak gain. According to the principle of pattern multiplication, the overall normalized gain of the array at a given look angle coincides with the normalized gain of the average active element gain pattern. Therefore, a uniform active element beam pattern leads to a uniform array beam pattern.

Polarization is the third key performance parameter to consider. Oftentimes, arrays exploit the advantages of orthogonal polarizations. This requires that radiating elements accommodate two separate feeds and maintain reasonable polarization purity. Some topologies more readily facilitate orthogonal feeds with moderate port-to-port isolation and a high degree of polarization purity.

\subsubsection{Profile}

In this application and for most tile-type arrays, form factor and cost are the primary driving constraints. These constraints have catalyzed adoption of manufacturing materials and processes developed and matured in the broader consumer electronics industry-namely printed circuit board manufacturing processes, surface mount technologies, and semiconductor devices. As a 
consequence, the radiating element trade space consists of planar antennas subjected to PCB process constraints.

Fortunately, printed antennas have been investigated thoroughly in the last three decades by academic, industrial, and government engineers. Over fifteen hundred papers and five books were published on the topic of printed microstrip antennas and arrays between 1980 and 1995. Most relevant to this work may be David Pozar's seminal work [63] on aperture-coupled microstrip patch antennas. Fundamentally, this work is an extension of the theoretical and experimental foundations established by Pozar and his contemporaries. There are a variety of books $[64,31$, 65, 66], patents [67, 68, 69, 70], and journal articles [71, 72, 73] providing consolidated overviews of progress made in the field of low-cost printed antenna design which have influenced the design topology selected in this work.

\subsubsection{Cost}

Minimizing the cost per radiating element is critical to achieving an affordable array consisting of thousands of radiating elements. To a large extent, modern arrays implemented as multilayer PCBs have successfully marginalized radiating element cost.

For example, consider an $\mathrm{X} / \mathrm{Ku}$-band satellite communications array consisting of a $1 \mathrm{~m}^{2}$ aperture filled with roughly 5,000 elements. The material cost allocated to the radiating elements represents $2-5 \%$ of the total bill of material $\operatorname{cost}^{9}$ for an entire array as illustrated in the material cost breakdown in Table 4.1.1. According to the table and corresponding pie charts given in Figure 4.1.1, radiating element cost marginalizes with increasing build quantity. At high quantity, material cost is dominated by amplifiers, RFICs, filters, and discrete cables and connectors, consistent with most tile-type arrays.

\footnotetext{
${ }^{9}$ This example is derived from a comprehensive bill of materials for a practical array system developed by
} the author. 
High frequency connectors and cables, which scale in quantity less advantageously than semiconductor devices and PCBs, are an active area of research for RF and millimeter wave applications. One relevant research thrust aims to achieve higher levels of integration - thereby minimizing interconnect complexity. Recent advancements in IBM's $45 \mathrm{~nm}$ and $32 \mathrm{~nm}$ siliconon-insulator (SOI) $[74,75]$ and $130 \mathrm{~nm} \mathrm{SiGe} \mathrm{BiCMOS} \mathrm{[76]} \mathrm{technologies} \mathrm{combined} \mathrm{with} \mathrm{options}$ for chip-scale packaging (CSP) and thru-silicon via (TSV) integration will enable higher levels of integration, lower cost, and lower power consumption for next generation mixed-signal products. A second research thrust aims to implement high frequency interconnects, filters, and passive devices using a wafer fabrication process that enjoys similar economy of scale encountered in semiconductor processes. One such example is the PolyStrata ${ }^{\circledR}$ process developed by Nuvotronics. In this wafer process, air-core coaxial transmission lines, chip transitions, filters, and other passive circuits are constructed with micron-level precision. This technology is expected to reduce cables and connectors and integrate filters or other passive devices in next generation RF and millimeter-wave antenna array products. 
Table 4.1.1 Bill of material cost for an X/Ku-band full-duplex satellite communications array illustrates marginalization of unit cell radiating element cost afforded by PCB implementation for three build quantities ${ }^{10}$. This table also highlights the nontrivial cost and unfavorable cost scaling over quantity for connectors and cables.

\begin{tabular}{clccc} 
ID & Material Category & $\begin{array}{c}\text { Low Qty } \\
\text { Cost }(\%)\end{array}$ & $\begin{array}{c}\text { Med. Qty } \\
\text { Cost }(\%)\end{array}$ & $\begin{array}{c}\text { High Qty } \\
\text { Cost }(\%)\end{array}$ \\
\hline 1 & Radiating Element PCBs & 5 & 3 & 1.5 \\
2 & RF Electronics PCBs & 6 & 4 & 2.5 \\
3 & DC Power \& Control PCBs & 3 & 1 & 0.5 \\
4 & Duplexers & 12 & 10 & 9 \\
5 & Bandpass Filters & 7 & 5 & 4 \\
6 & RF Connectors \& Cables & 20 & 27 & 32 \\
7 & Amplifiers & 27 & 32 & 35 \\
8 & Beamforming RFICs & 14 & 12 & 10 \\
9 & Mechanical Structures & 2 & 2 & 1.5 \\
10 & Other $^{11}$ & 4 & 4 & 4 \\
\hline
\end{tabular}
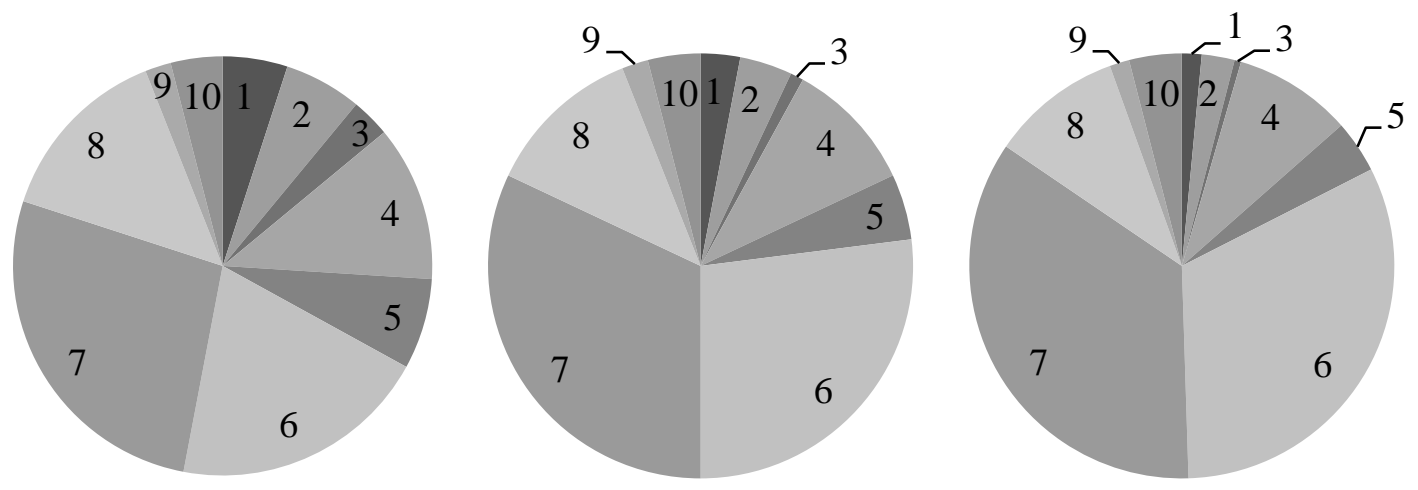

Figure 4.1.1 Visualization of a bill of material cost breakdown for an X/Ku-band full-duplex satellite communications array in low, medium, and high volume production.

\footnotetext{
${ }^{10}$ In this example, the difference between each quantity listed is one order of magnitude.

${ }^{11}$ Includes electronics enclosures, panel alignment features, thermal control hardware, weatherproofing materials, consumable materials, and a protective radome.
} 


\subsection{Prior Design}

A unit cell antenna has been designed, fabricated, and tested on a prior effort for a $\mathrm{X} / \mathrm{Ku}$-band satellite communications array application. This design employs stacked circular patch radiators fed by capacitive-coupled probe feeds followed by a single stage branchline coupler implemented in a multilayer PCB. This section describes the requirements, topology, electromagnetic model, and simulated performance of the design.

\subsubsection{Requirements}

An array comprised of many unit cell antennas must operate in a shared aperture configuration and in full-duplex mode from 10.7 to $14.5 \mathrm{GHz}$ with return loss in excess of $12 \mathrm{~dB}$. An operating bandwidth of $3.8 \mathrm{GHz}$ represents a fractional bandwidth of $30.2 \%$. This performance must be maintained in an active array environment for all scan angles and for realistic materials and manufacturing process tolerances. To ensure compliance, it is wise to select antenna and hybrid topologies that achieve a slightly wider operational bandwidth to avoid non-compliance arising from performance degradation caused by materials and manufacturing process tolerances.

The size of the unit cell antenna is derived from the required grating lobe free scan volume and the size constraints of the full aperture. For this application, a scan limit of $45^{\circ}$ in elevation is desired; however, the prior design first aimed to achieve $30^{\circ}$ to mitigate risk associated with the integration of beamforming electronics and interconnects in a compressed lattice capable of a $45^{\circ}$ scan. At the maximum frequency of operation, the size of the unit cell must be 0.541 " by 0.469 " to achieve a $47.5^{\circ}$ scan, which includes a $2.5^{\circ}$ angular buffer to ensure the entire grating lobe does not enter real space for a beam scanned to $45^{\circ}$.

Realized gain represents the directivity reduced by the total efficiency. For this application, realized gain must exceed $4.2 \mathrm{dBi}$ from 10.7 to $12.7 \mathrm{GHz}$ and $5.5 \mathrm{dBi}$ from 13.7 to $14.5 \mathrm{GHz}$. 
Additionally, port-to-port isolation must exceed $20 \mathrm{~dB}$ and cross-polarization level cannot exceed $-17 \mathrm{~dB}$.

To achieve dual-circular polarization from a dual-linearly polarized antenna, the unit cell design requires a $90^{\circ}$ hybrid. The hybrid must integrate into the unit cell size of the antenna and operate over the same bandwidth. Port return loss and port-to-port isolation should exceed $15 \mathrm{~dB}$, and insertion loss must remain below $0.4 \mathrm{~dB}$. Lastly, the design is allocated 0.8 degrees and 0.2 $\mathrm{dB}$ from the RMS error budget for phase and amplitude error, respectively.

Recall, RMS phase error is defined by

$$
\begin{aligned}
& \varepsilon_{\text {phase }, \text { rms }} \\
& =\sqrt{\frac{1}{n} \sum_{k=1}^{n}\left([\arg \mathrm{S} 21-\arg S 31]_{k}-\frac{1}{n} \sum_{m=1}^{n}[\arg S 21-\arg S 31]_{m}\right)^{2}} \text { (deg. ) }
\end{aligned}
$$

where $[\arg S 21-\arg S 31]_{i}$ represents the phase difference between hybrid output ports at the $i$ th frequency point within the operating bandwidth. Similarly, RMS amplitude error is defined by

$$
\begin{aligned}
& \varepsilon_{\text {amp }, r m s} \\
& =\sqrt{\frac{1}{2 n}\left[\sum_{k=1}^{n}\left(|S 21|_{k}-\frac{1}{n} \sum_{m=1}^{n}|S 21|_{m}\right)^{2}+\sum_{k=1}^{n}\left(|S 31|_{k}-\frac{1}{n} \sum_{m=1}^{n}|S 31|_{m}\right)^{2}\right]}
\end{aligned}
$$


where $|S 21|_{i}$ represents the amplitude difference between hybrid output ports at the $i$ th frequency point within the operating bandwidth. The requirements for the unit cell antenna and hybrid design are tabulated in Table 4.2.1.

Table 4.2.1 Requirements for the dual-circularly polarized $\mathrm{X} / \mathrm{Ku}$-band unit cell antenna and hybrid. Realized antenna gain expressed in units of $\mathrm{dBic}$ represents gain for circular polarization assuming the dual-linearly polarized antenna is fed with an ideal (lossless) $90^{\circ}$ hybrid coupler.

\begin{tabular}{lccc} 
Antenna Parameter & $</>$ & Req. & $\mathrm{Units}$ \\
\hline Min. Operational Frequency & $=$ & 10.7 & $\mathrm{GHz}$ \\
Max. Operational Frequency & $=$ & 14.5 & $\mathrm{GHz}$ \\
Port Reflection Coefficient & $<$ & -10 & $\mathrm{~dB}$ \\
Realized Gain, 10.7-12.7 GHz & $>$ & 4.2 & $\mathrm{dBic}$ \\
Realized Gain, 13.7-14.5 GHz & $>$ & 5.5 & $\mathrm{dBic}$ \\
Port-to-Port Coupling & $<$ & -20 & $\mathrm{~dB}$ \\
Cross-Pol. Level & $<$ & -17 & $\mathrm{~dB}$ \\
Unit Cell Size, X-dimension & $=$ & 541 & $\mathrm{mil}$ \\
Unit Cell Size, Y-dimension & $=$ & 469 & $\mathrm{mil}$ \\
& & & \\
Hybrid Parameter & $</>$ & $\mathrm{Req}$. & $\mathrm{Units}$ \\
\hline Min. Operational Frequency & $=$ & 10.7 & $\mathrm{GHz}$ \\
Max. Operational Frequency & $=$ & 14.5 & $\mathrm{GHz}$ \\
Port Reflection Coefficient & $<$ & -15 & $\mathrm{~dB}$ \\
Port-to-Port Coupling & $<$ & -15 & $\mathrm{~dB}$ \\
Insertion Loss & $<$ & 0.5 & $\mathrm{~dB}$ \\
RMS Phase Error & $<$ & 0.8 & $\mathrm{deg}$. \\
RMS Amplitude Error & $<$ & 0.2 & $\mathrm{~dB}$ \\
Unit Cell Size, X-dimension & $=$ & 541 & $\mathrm{mil}$ \\
Unit Cell Size, Y-dimension & $=$ & 469 & $\mathrm{mil}$ \\
\hline
\end{tabular}




\subsubsection{Topology}

The unit cell antenna consists of stacked circular patch radiators with a mode suppression center ground via. Two orthogonal capacitively-coupled probes feed the bottom patch radiator. A single-stage branchline coupler in stripline implements the $90^{\circ}$ hybrid required to convert the dual-linearly polarized antenna to dual-circular polarization. A sketch of the antenna model and its key dimensions is provided in Figure 4.2.1.

A parameterized electromagnetic model constructed in CST Microwave Studio facilitates parametric optimization of the antenna geometry. Figure 4.2.2 illustrates this parameterized electromagnetic model. Microwave Studio includes solvers capable of computing solutions in both the time and frequency domains. The time domain solver employs the Finite-Difference Time-Domain Method (FDTD), and the frequency domain solver employs the Finite Element Method (FEM). For structures comprised of many dielectric layers operating over a wide bandwidth, the time domain solver is generally favored [56]. Following design optimization in the time-domain solver, it is worthwhile to verify the design using the frequency-domain solver, and if time permits, other analysis software packages such as Ansys HFSS or FEKO.

A comparison between the EM model and an x-ray image of the fabricated unit cell antenna is provided in Figure 4.2.3. X-ray imaging is employed to validate the manufactured design, assess craftsmanship, and troubleshoot problems. The multilayer PCB in the x-ray image contains the 7 layers for the antenna and hybrid and additional layers for passive combiners, digital control signal routing, ground planes, and land patterns for solder-attached electronics packages.

A separate ADS model facilitates parametric optimization of the single-stage branchline hybrid coupler. This model is illustrated in Figure 4.2.4. As will be discussed in the next section, once the hybrid solution is optimized in ADS and Momentum, it transitions to CST Microwave Studio for final optimization and integration with the antenna model. 


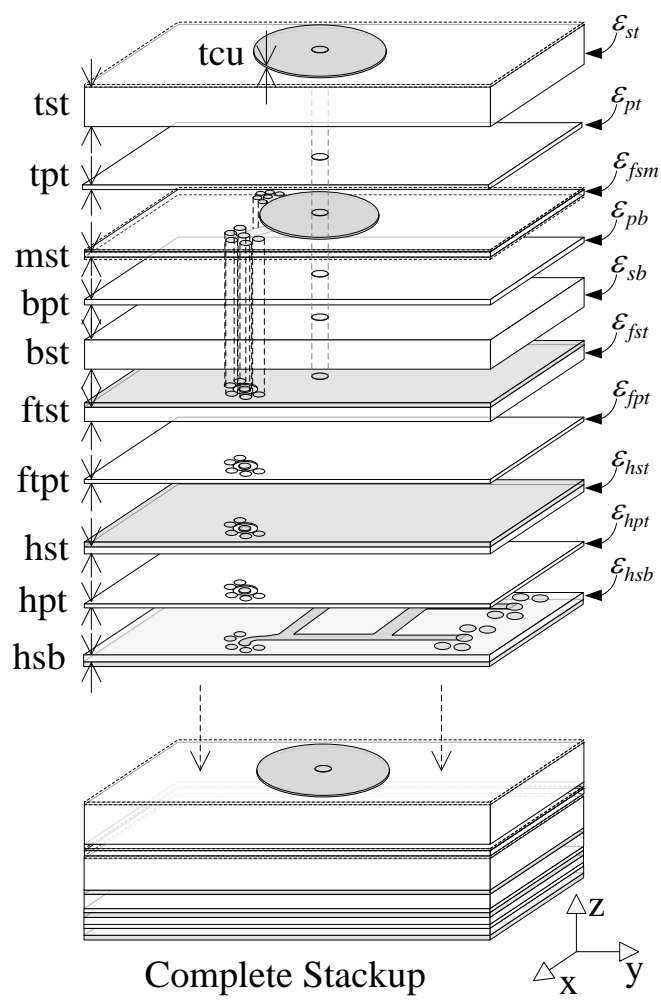

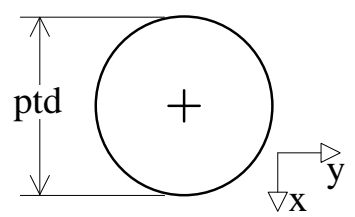

Top Patch

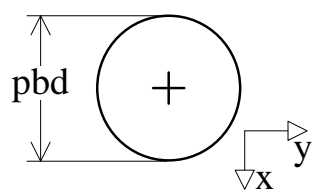

Bottom Patch

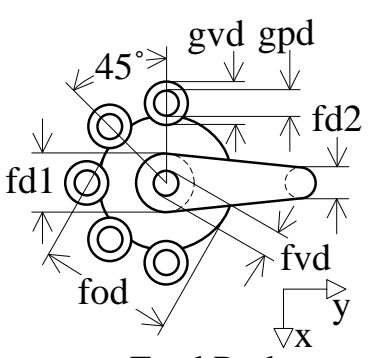

Feed Probe
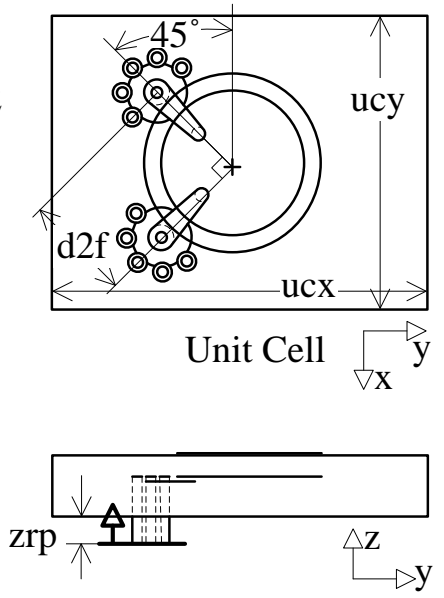

EM Model Reference Planes

Figure 4.2.1 The parameterized prior unit cell antenna model constructed in CST Microwave Studio. The hybrid appears in the stackup, but is modeled and simulated separately in Agilent ADS using Momentum. Drawing is not to scale. 


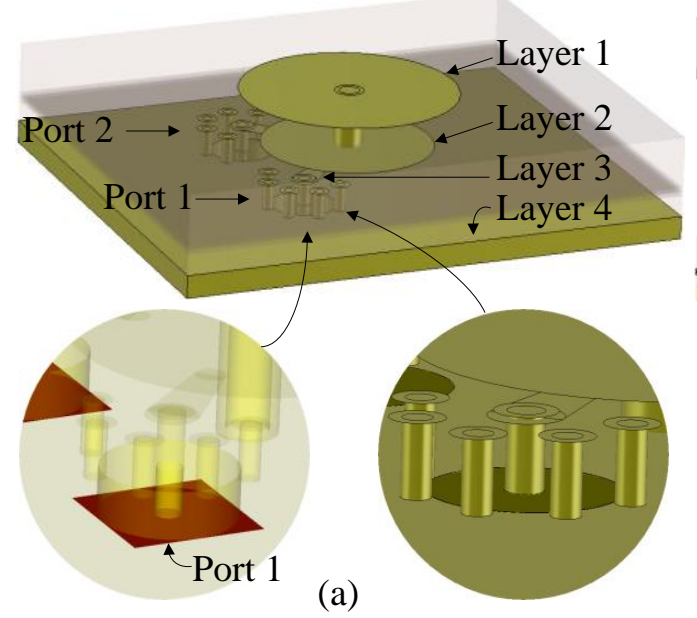

(a)

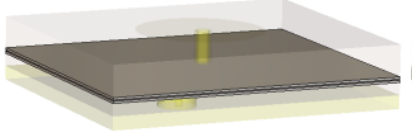

(d)

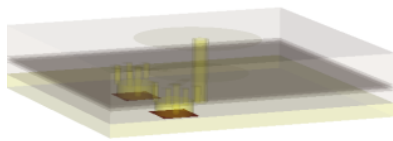

(f)

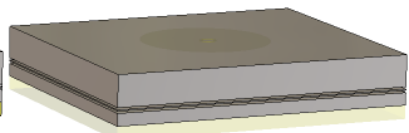

(c)

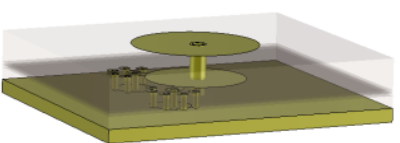

(e)

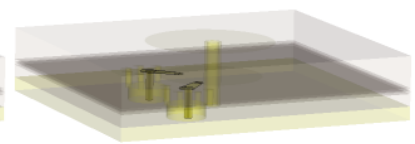

$(\mathrm{g})$

Figure 4.2.2 The prior unit cell antenna design constructed in CST Microwave Studio illustrating (a) stackup layers with ports and feeds, (b) full stackup, (c) substrates only, (d) prepregs only, (e) metallic layers only, (f) waveguide ports, and $(\mathrm{g})$ feed probe vias.

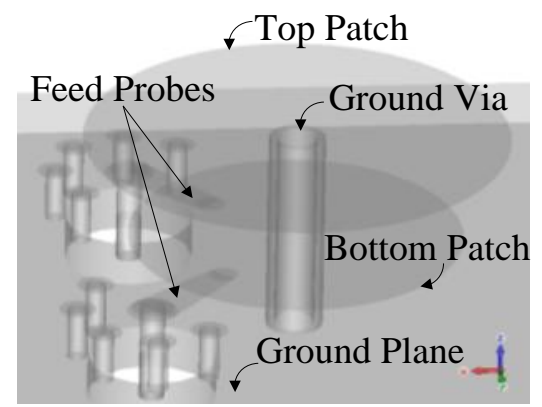

(a)

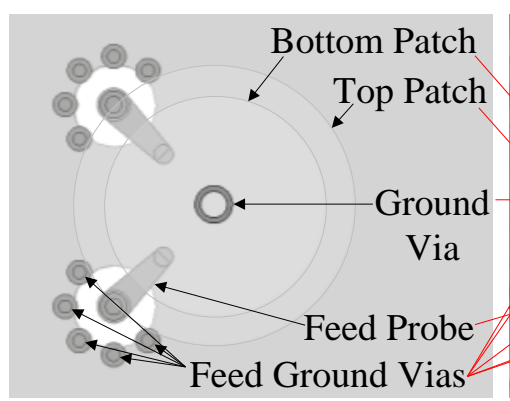

(b)

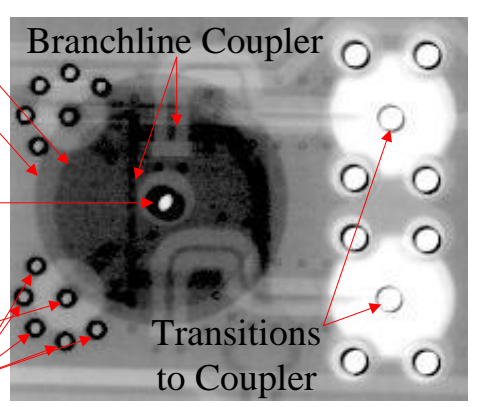

(c)

Figure 4.2.3 (a-b) Two views of the prior unit cell antenna model constructed in CST Microwave Studio and (c) an x-ray image of the fabricated unit cell antenna within a multilayer PCB. The $\mathrm{x}$-ray image also includes the single stage hybrid as well as transitions and traces from other layers in the stackup. 


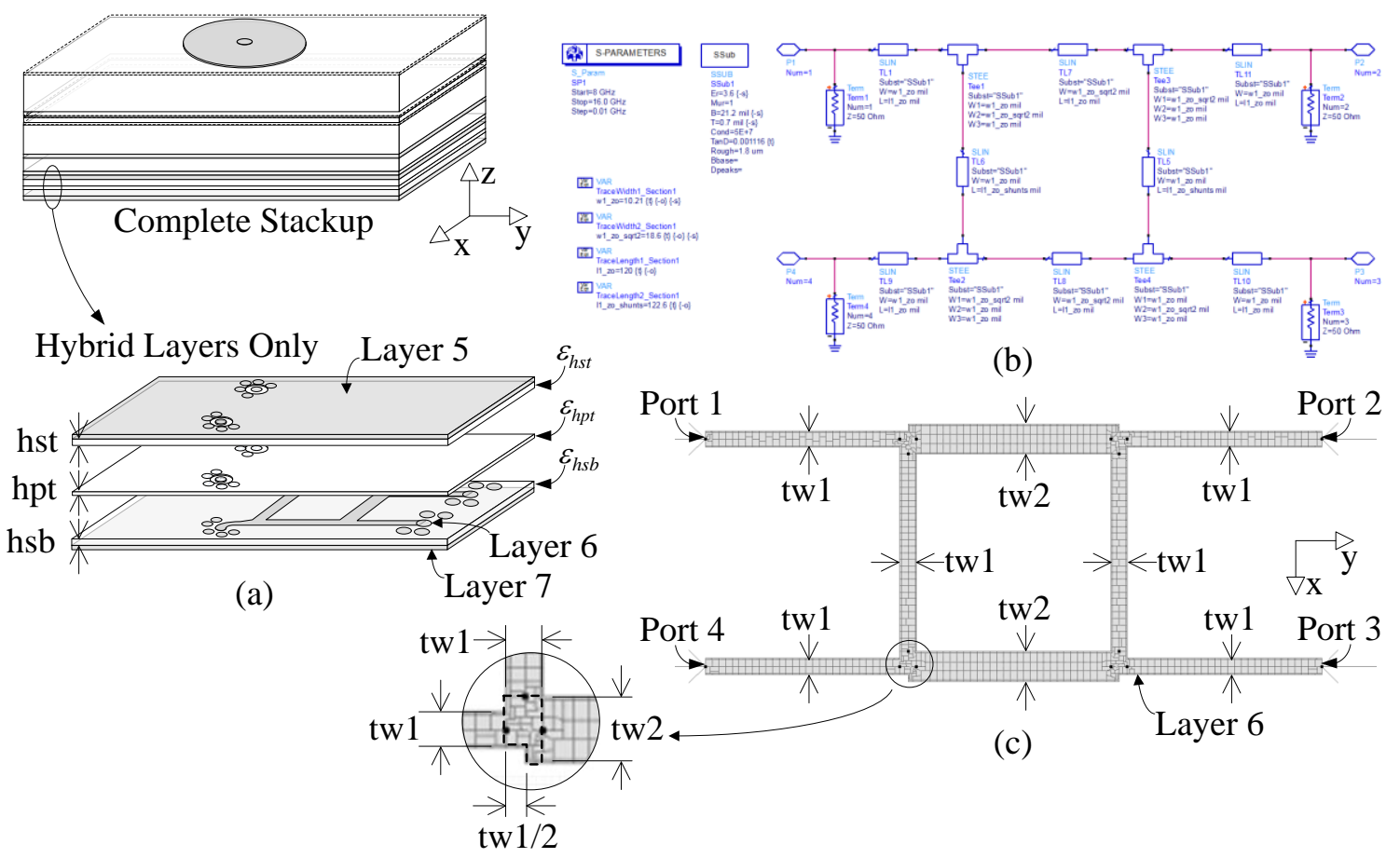

Figure 4.2.4 The parameterized model of the prior single-stage branchline coupler design illustrating (a) the hybrid portion of the stackup, (b) the ADS schematic used to optimize the design, and (c) the meshed ADS layout. The transition vias between the hybrid and antenna are not included in the model.

\subsubsection{Performance}

The performance of the prior unit cell antenna design does not satisfy many of the requirements. For brevity, all performance curves will be included in the next section which describes the improved antenna and compares the two designs. Table 4.2.2 summarizes performance and highlights deficiencies. The requirements in the table were not defined for multiple sub-bands within the operating bandwidth, with the exception of realized gain, so the table captures the very poor performance exhibited in the lower portion of the band. However, the design performs reasonably well in the upper portion of the band. 
Table 4.2.2 Prior unit cell antenna and hybrid simulated performance compared to the requirements. Nominal values for material properties and model dimensions are assumed.

\begin{tabular}{lcc|c|c} 
& & & Prior & \\
Antenna Parameter & $</>$ & Req. & Design & Units \\
\hline Min. Operational Frequency & $=$ & 10.7 & 10.7 & $\mathrm{GHz}$ \\
Max. Operational Frequency & $=$ & 14.5 & 14.5 & $\mathrm{GHz}$ \\
Port Reflection Coefficient & $<$ & -10 & -13 & $\mathrm{~dB}$ \\
Realized Gain, 10.7-12.7 GHz & $>$ & 4.2 & $\mathbf{0 . 4}$ & $\mathrm{dBic}$ \\
Realized Gain, 13.7-14.5 GHz & $>$ & 5.5 & $\mathbf{4 . 9}$ & $\mathrm{dBic}$ \\
Port-to-Port Coupling & $<$ & -20 & $\mathbf{- 5 . 0}$ & $\mathrm{dB}$ \\
Cross-Pol. Level & $<$ & -17 & $\mathbf{- 5 . 3}$ & $\mathrm{dB}$ \\
Unit Cell Size, X-dimension & $=$ & 541 & $\mathbf{6 0 3}$ & $\mathrm{mil}$ \\
Unit Cell Size, Y-dimension & $=$ & 469 & $\mathbf{5 2 2}$ & $\mathrm{mil}$ \\
& & & & \\
Hybrid Parameter & $</>$ & Req. & Design & $\mathrm{Units}$ \\
\hline Min. Operational Frequency & $=$ & 10.7 & 10.7 & $\mathrm{GHz}$ \\
Max. Operational Frequency & $=$ & 14.5 & 14.5 & $\mathrm{GHz}$ \\
Port Reflection Coefficient & $<$ & -15 & $\mathbf{- 9 . 8}$ & $\mathrm{dB}$ \\
Port-to-Port Coupling & $<$ & -15 & $\mathbf{- 9 . 8}$ & $\mathrm{dB}$ \\
Insertion Loss & $<$ & 0.5 & $\mathbf{0 . 9 5}$ & $\mathrm{dB}$ \\
RMS Phase Error & $<$ & 0.8 & $\mathbf{1 . 4 0 2}$ & $\mathrm{deg}$. \\
RMS Amplitude Error & $<$ & 0.2 & $\mathbf{0 . 2 9 7}$ & $\mathrm{dB}$ \\
Unit Cell Size, X-dimension & $=$ & 541 & $\mathbf{6 0 3}$ & $\mathrm{mil}$ \\
Unit Cell Size, Y-dimension & $=$ & 469 & $\mathbf{5 2 2}$ & $\mathrm{mil}$ \\
\hline
\end{tabular}

\subsubsection{Challenges and Improvements}

There are three primary causes degrading performance of the prior unit cell design. The first cause relates to the feed. For capacitively-coupled probe feeds, it is challenging to simultaneously optimize bandwidth and port match. Optimizing bandwidth involves tuning patch diameters and substrate thicknesses. Generally, thicker substrates will increase bandwidth. Varying substrate thickness also lengthens the probe feeds and thereby increases inductive reactance, requiring compensation achieved by varying feed probe dimensions. For this reason, designs reported in Literature for capacitively-coupled stack patches are typically limited to bandwidth below $25 \%$. Moreover, the close proximity of the capacitively-coupled feed to the radiating patch tends to squint the element pattern and degrade its uniformity. As an alternative, an aperture-coupled feed 
may provide some decoupling between the feed design and overall antenna performance. Designs employing this approach in Literature confirm the merits of decoupling and achieve $25-40 \%$ bandwidth [72, 77, 78, 71, 79].

The second cause of poor performance relates to the selection of a single stage for the branchline coupler. It is well known that a single-stage branchline coupler exhibits a $10 \mathrm{~dB}$ return loss bandwidth close to $30 \%$, but the insertion loss as well as the amplitude and phase imbalance degrades appreciably beyond a bandwidth of $18-20 \%$. Therefore, useful operation is limited to a maximum bandwidth of $18-20 \%$.

A multi-stage branchline coupler may provide the required impedance bandwidth and amplitude and phase balance. The challenge in a multi-stage design lies in the ability to fit the design within the size limitations of the array grid. To make the design more compact, one can increase substrate dielectric constant and decrease substrate thickness. However, increasing dielectric constant of one substrate in a multilayer stackup complicates interlayer transition design. A higher dielectric constant substrate also tends to have a higher dielectric loss tangent. Lastly, an increased dielectric constant and decreased substrate thickness generally leads to decreased transmission line width. Reduced trace width leads to increased loss per unit length of the transmission lines used to implement the hybrid, and thinner substrates tend to reduce robustness of the design to materials and manufacturing tolerances. Additional stages also increases insertion loss due to lengthened signal paths. Therefore, the number of sections in a multi-stage coupler is a design trade between bandwidth and insertion loss.

This prior unit cell antenna design was fabricated and tested. Measured performance weakly correlates with simulated performance. As will be discussed in the design comparison section, weak correlation between simulation and measurement is likely attributed to variance associated with material property and manufacturing process tolerances. A capacitive feed is very sensitive to misalignment as well as the dielectric constant and thickness of the substrate between the feed 
probe and the patch. An improved design must consider sensitivity to materials and manufacturing processes as an integral part of the design and downselection process. 


\subsection{Preliminary Design}

An improved design that addresses the challenges encountered in the prior design is proposed in this section.

\subsubsection{Antenna Topology}

The preliminary unit cell consists of an antenna layer comprised of stacked square patch radiators, a feed layer comprised of two orthogonal aperture-coupled stripline feeds, and a beamforming layer containing a hybrid coupler and other passive devices as described in Figure 4.3.1. This section describes the antenna and feed layers, and section 4.3 .3 describes the hybrid section of the beamforming layer. A sketch of the antenna model and its key parameters is illustrated in Figure 4.3.2, and the electromagnetic model constructed in Microwave Studio is pictured in Figure 4.3.3.

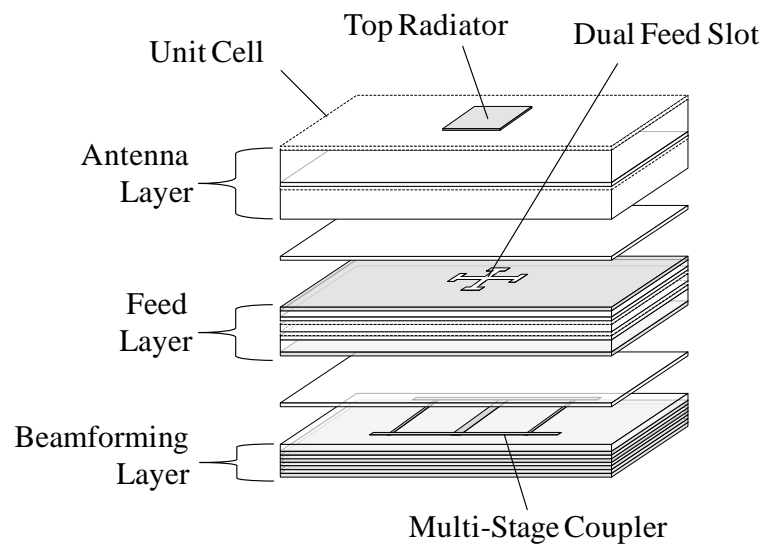

Figure 4.3.1 The unit cell antenna topology consists of an antenna layer, feed layer, and beamforming layer. The antenna layer contains the first two metal layers in the stackup as well as dielectric substrates. The feed layer contains the next four metal layers. The beamforming layer contains three more layers for the hybrid and a few other layers for Wilkinson power splitters and subarray-level signal routing. This work includes only the hybrid portion of the beamforming layer. 

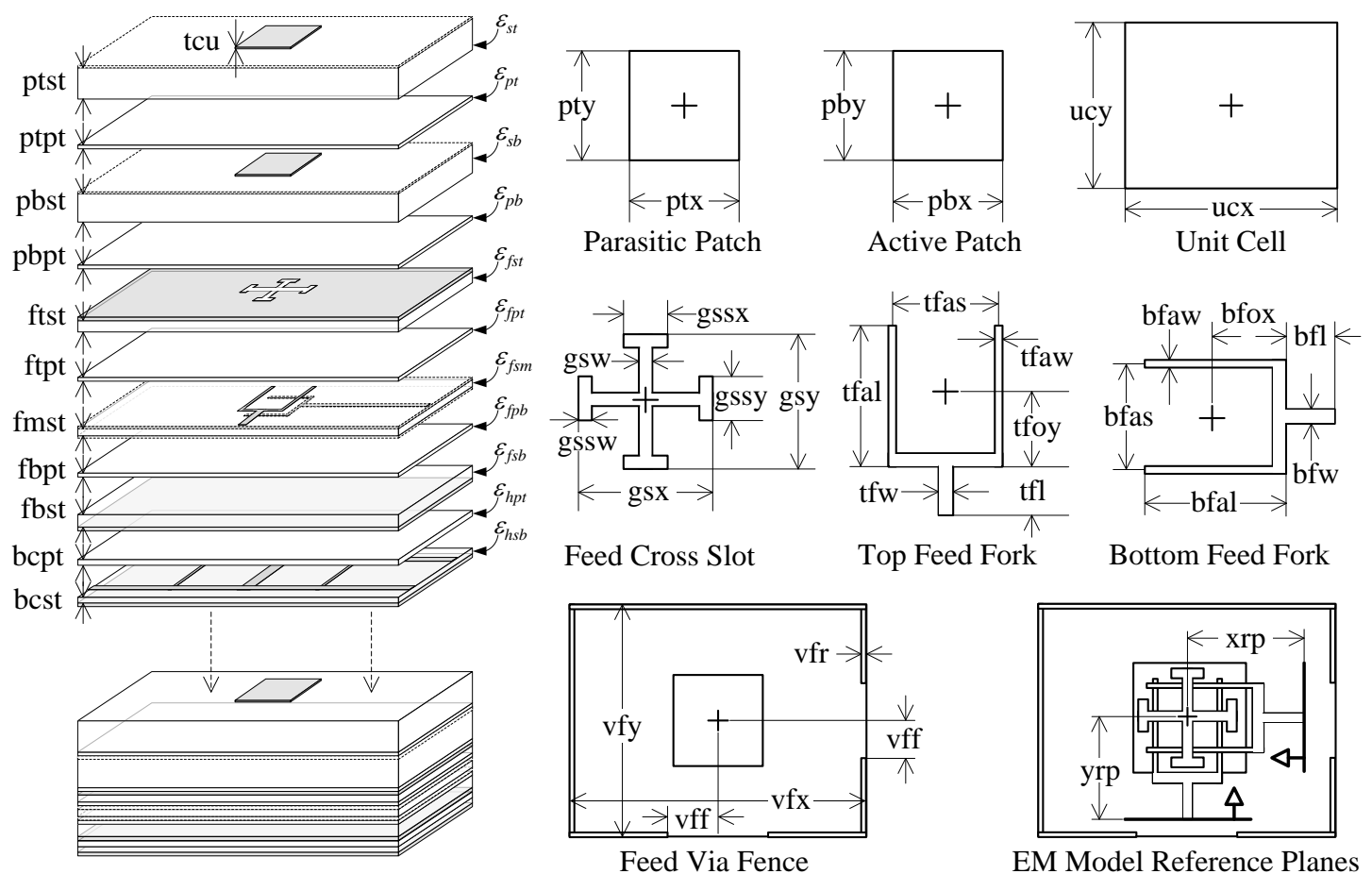

Feed Cross Slot

Top Feed Fork

Bottom Feed Fork

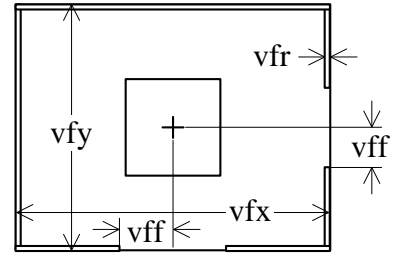

Feed Via Fence

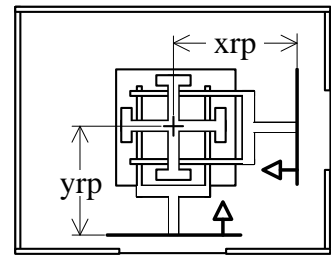

EM Model Reference Planes

Figure 4.3.2 The proposed preliminary unit cell antenna model constructed in CST Microwave Studio. The hybrid appears in the stackup, but is modeled and simulated separately in Agilent ADS using Momentum. Drawing is not to scale.

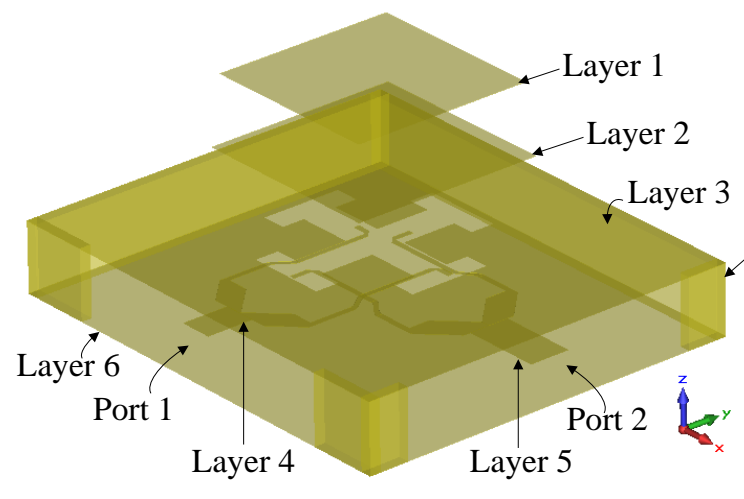

(a)

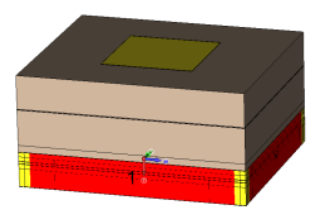

(b)

Wall

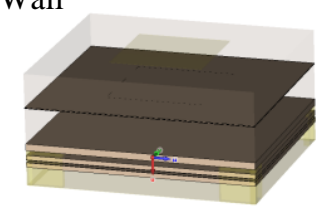

(d)

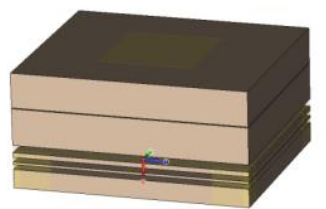

(c)

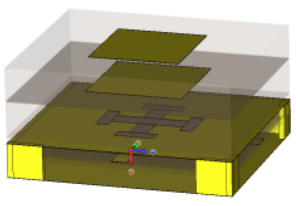

(e)

Figure 4.3.3 The preliminary unit cell antenna design constructed in CST Microwave Studio illustrating (a) all six metal layers, (b) stackup layers with ports and feeds, (c) substrates only, (d) prepregs and bond plies only, and (e) metallic layers only. The stripline feed region is enclosed with PEC walls to increase isolation between adjacent feeds and to suppress parallel plate modes. Two sides of the wall are bifurcated to accommodate waveguide ports. Accurate via structures will replace the PEC wall in the final design. 


\subsubsection{Antenna Performance}

The preliminary antenna satisfies many of the requirements as summarized in Table 4.3.1. Compared with the prior design, the proposed preliminary design utilizes $19 \%$ less area and has 2-3 dB higher average return loss, $1 \mathrm{~dB}$ higher average boresight gain, $3 \mathrm{~dB}$ lower average crosspolarization level, $10 \mathrm{~dB}$ higher average port isolation, and improved radiation pattern uniformity. Simulation curves with detailed captions are presented in Figure 4.3.4, Figure 4.3.5, Figure 4.3.6, Figure 4.3.7, and Figure 4.3.8.

Table 4.3.1 Performance comparison of the preliminary and prior antenna and hybrid designs compared to the requirements. Nominal values for material properties and model dimensions are assumed.

\begin{tabular}{lcc|c|c|c} 
& & & Prior & Prelim. & \\
Antenna Parameter & $</>$ & Req. & Design & Design & Units \\
\hline Min. Operational Frequency & $=$ & 10.7 & 10.7 & 10.7 & $\mathrm{GHz}$ \\
Max. Operational Frequency & $=$ & 14.5 & 14.5 & 14.5 & $\mathrm{GHz}$ \\
Port Reflection Coefficient & $<$ & -10 & -13 & -16 & $\mathrm{~dB}$ \\
Realized Gain, 10.7-12.7 GHz & $>$ & 4.2 & $\mathbf{0 . 4}$ & 4.3 & $\mathrm{dBic}$ \\
Realized Gain, 13.7-14.5 GHz & $>$ & 5.5 & $\mathbf{4 . 9}$ & $\mathbf{6 . 0}$ & $\mathrm{dBic}$ \\
Port-to-Port Coupling & $<$ & -20 & $\mathbf{- 5 . 0}$ & $\mathbf{- 1 7}$ & $\mathrm{dB}$ \\
Cross-Pol. Level & $<$ & -17 & $\mathbf{- 5 . 3}$ & $-\mathbf{1 5}$ & $\mathrm{dB}$ \\
Unit Cell Size, X-dimension & $=$ & 541 & $\mathbf{6 0 3}$ & $\mathbf{5 4 1}$ & $\mathrm{mil}$ \\
Unit Cell Size, Y-dimension & $=$ & 469 & $\mathbf{5 2 2}$ & 469 & $\mathrm{mil}$ \\
& & & Prior & Prelim. & \\
Hybrid Parameter & $</>$ & Req. & Design & Design & $\mathrm{Units}$ \\
\hline Min. Operational Frequency & $=$ & 10.7 & 10.7 & 10.7 & $\mathrm{GHz}$ \\
Max. Operational Frequency & $=$ & 14.5 & 14.5 & 14.5 & $\mathrm{GHz}$ \\
Port Reflection Coefficient & $<$ & -15 & $\mathbf{- 9 . 8}$ & -22 & $\mathrm{~dB}$ \\
Port-to-Port Coupling & $<$ & -15 & $\mathbf{- 9 . 8}$ & -22 & $\mathrm{~dB}$ \\
Insertion Loss & $<$ & 0.5 & $\mathbf{0 . 9 5}$ & $\mathbf{0 . 3 0}$ & $\mathrm{dB}$ \\
RMS Phase Error & $<$ & 0.8 & $\mathbf{1 . 4 0 2}$ & $\mathbf{0 . 1 8 8}$ & $\mathrm{deg}$. \\
RMS Amplitude Error & $<$ & 0.2 & $\mathbf{0 . 2 9 7}$ & $\mathbf{0 . 1 1 3}$ & $\mathrm{dB}$ \\
Unit Cell Size, X-dimension & $=$ & 541 & $\mathbf{6 0 3}$ & $\mathbf{5 4 1}$ & $\mathrm{mil}$ \\
Unit Cell Size, Y-dimension & $=$ & 469 & $\mathbf{5 2 2}$ & $\mathbf{4 6 9}$ & $\mathrm{mil}$ \\
\hline
\end{tabular}




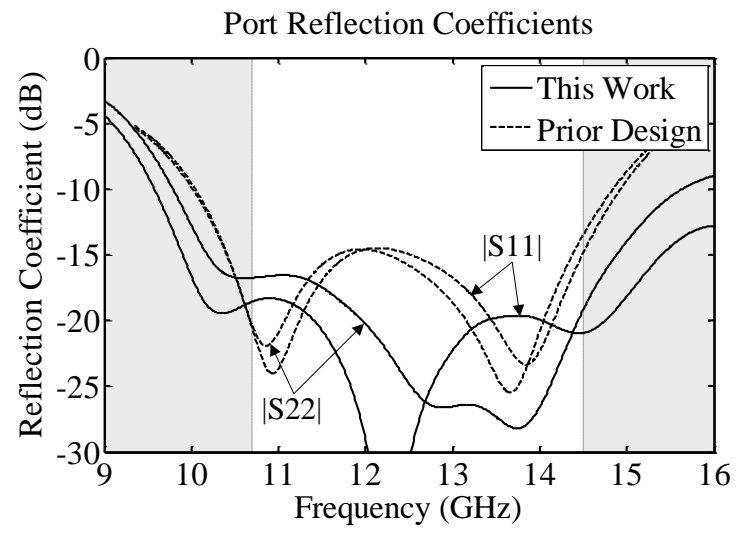

(a)

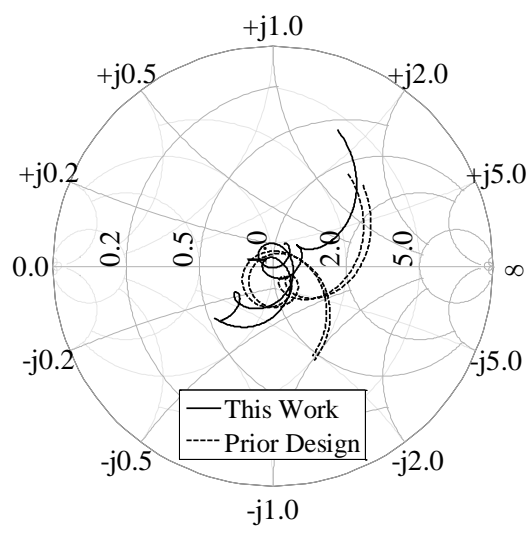

(b)

Figure 4.3.4 Port reflection coefficients for the preliminary and prior designs. Both designs are reasonably well matched for this application. The preliminary design has an average of roughly $2 \mathrm{~dB}$ better return loss than the prior design.

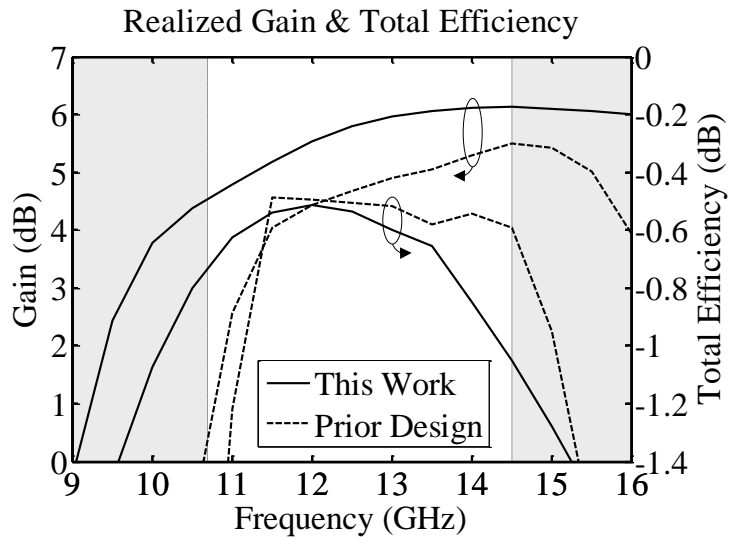

Figure 4.3.5 Comparison of boresight gain and total efficiency versus frequency for the preliminary and prior designs. Notice the preliminary design improves boresight gain by roughly $1 \mathrm{~dB}$ across the operational band. Both designs exhibit similar efficiency; the preliminary design is slightly more efficient in the lower band and slightly less efficient in the upper band. 


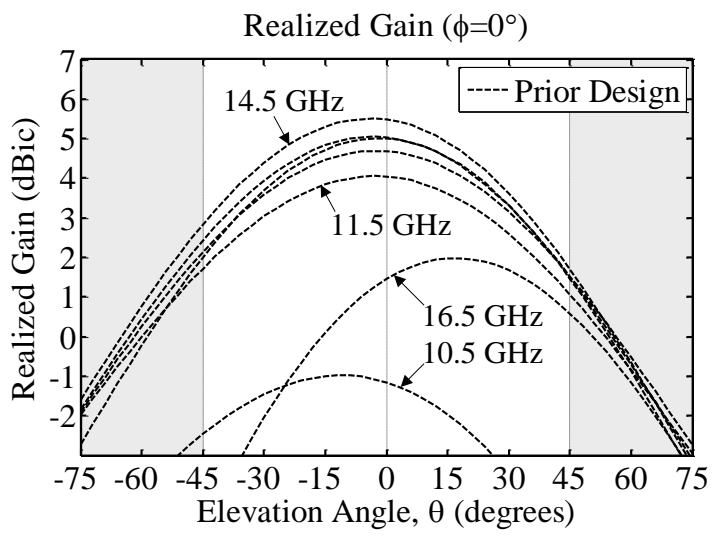

(a)

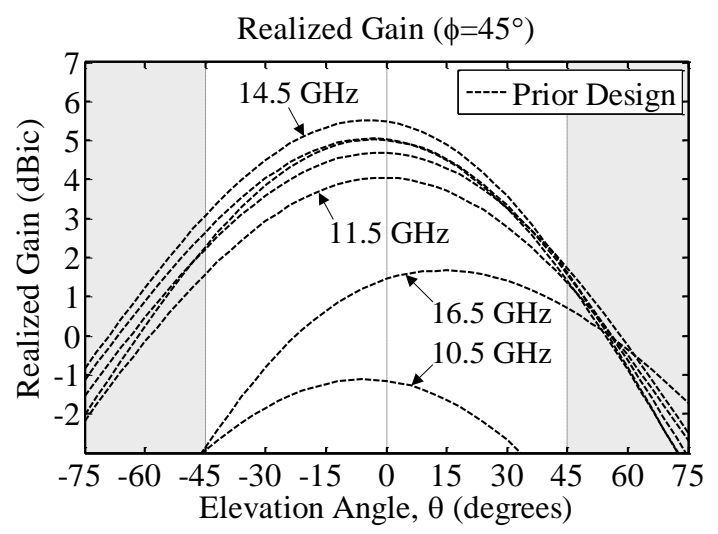

(c)

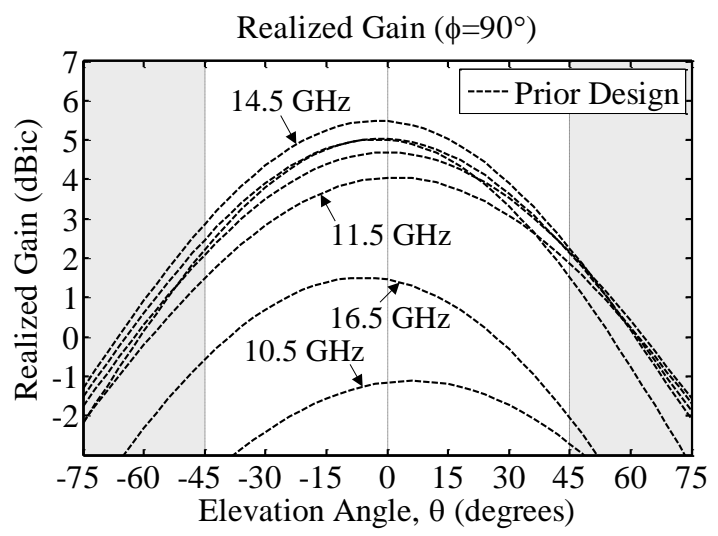

(e)

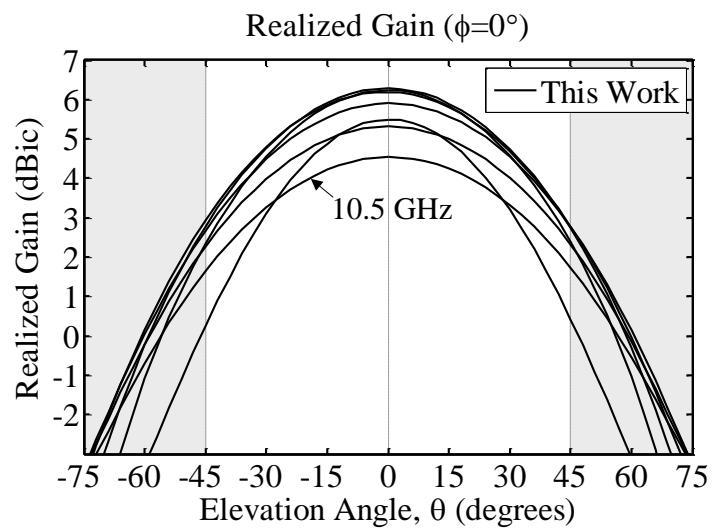

(b)

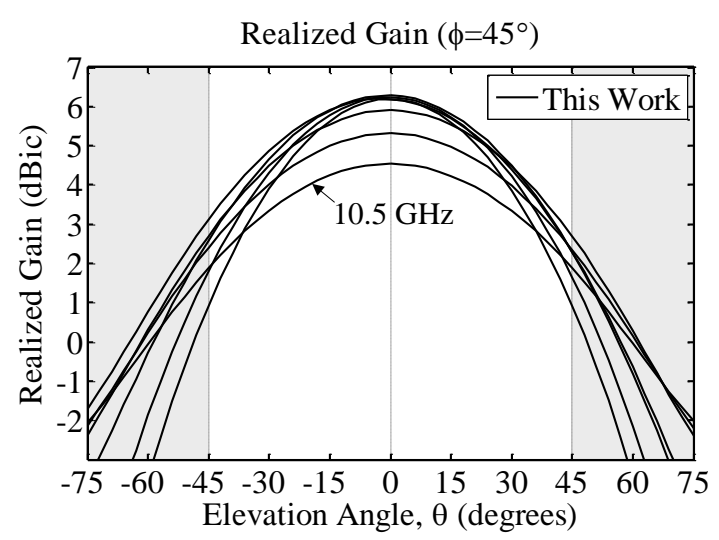

(d)

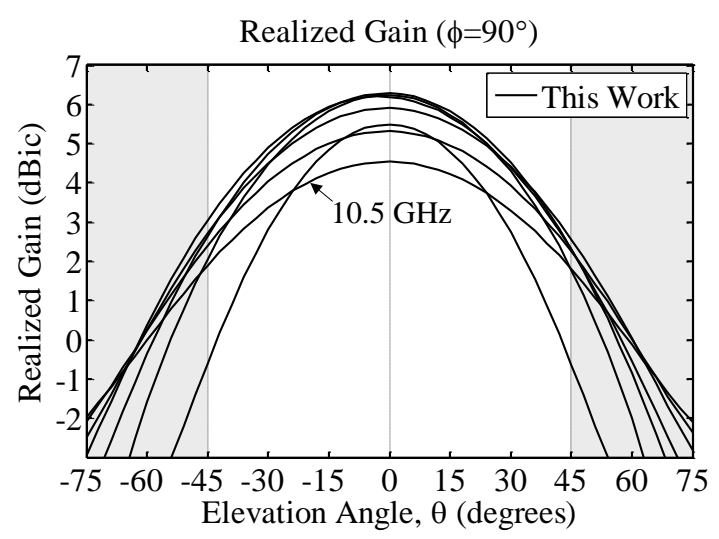

(f)

Figure 4.3.6 Comparison of 2D theta cuts of the 3D radiation patterns. Plots on the left column - (a), (c), and (e) - correspond to the prior design. Plots on the right column - (b), (d), and (f) - correspond to the preliminary design. The top, middle, and bottom rows correspond to $\phi=0^{\circ}, 45^{\circ}, 90^{\circ}$, respectively. Curves for seven frequencies are given on each plot, uniformly spaced from 10.5 to 16.5 GHz. The preliminary design overcomes the slight non-symmetry, steep gain roll-off versus frequency, and lower peak gain exhibited by the prior design. 

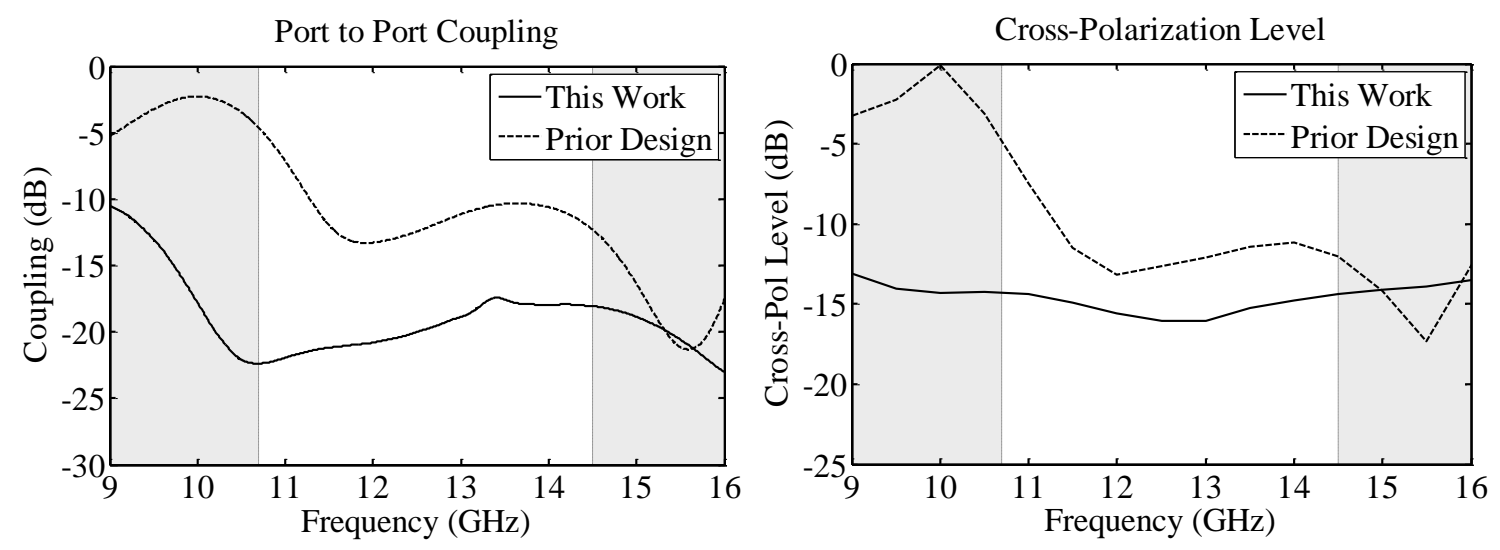

Figure 4.3.7 Comparison of port-to-port coupling and cross-polarization level of the preliminary and prior design. The preliminary design reduces coupling by an average of roughly $10 \mathrm{~dB}$ and reduces cross-polarization level by an average of 3 $\mathrm{dB}$. Coupling is less than $-17 \mathrm{~dB}$, and cross-polarization level is less than $-15 \mathrm{~dB}$ across the operating band.

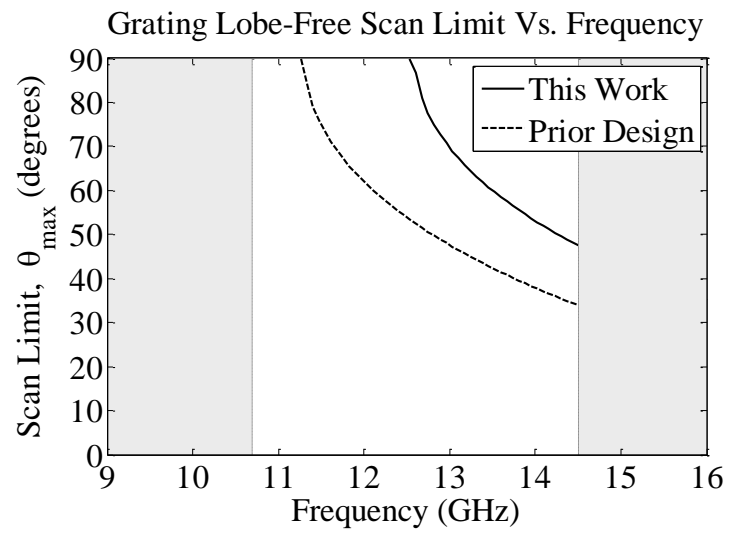

Figure 4.3.8 Comparison of grating-lobe free scan limit of the preliminary antenna design and the prior design. Grating-lobe free scan range is related to the dimensions of the unit cell. The proposed design utilizes a 19\% smaller area and consequently has an extended scan range. The design allows for $47.5^{\circ}$ gratinglobe free scan range at $14.5 \mathrm{GHz}$, which includes a buffer of $2.5^{\circ}$ to ensure the entire grating lobe of a large array does not enter real space. 


\subsubsection{Hybrid Topology}

As discussed in section 4.2.4, the single-stage branchline coupler exhibits an impedance bandwidth close to $30 \%$. However, phase and amplitude imbalance are degraded at band edges, so the useful fractional bandwidth is $18-20 \%$. Adding a stage to the design using well known design methods [59] increases the impedance bandwidth to 55\% and useful fractional bandwidth to roughly $40 \%$. This topology satisfies the bandwidth requirements of the application with reasonable margin. Therefore, a stripline dual-stage branchline coupler is selected to realize the $90^{\circ}$ hybrid required to convert the dual-linearly polarized antenna to dual-circular polarization.

A three-stage design was also considered, but requires complicated meandering of transmission lines and substrates with higher dielectric constant to fit within the size constraints. These conditions result in higher insertion loss. The hybrid is encountered before the LNA, so losses directly degrade system noise temperature of the receive chain and overall $\mathrm{G} / \mathrm{T}$ of the receive subsystem. To regain $\mathrm{G} / \mathrm{T}$ compliance, one can increase the aperture size. This leads to increased overall cost, weight, and DC power consumption. Higher insertion loss after the PA also reduces the radiated power per element and therefore degrades EIRP of the transmit subsystem. To regain EIRP compliance, one can redesign the PA for maximum power added efficiency (PAE) at a higher output power; this increases overall DC power consumption and increases power dissipation (i.e. generates more heat). Therefore, the three-stage design was eliminated from the trade space.

\subsubsection{Hybrid Performance}

An ADS model was employed to tune the dual-stage branchline hybrid coupler. This model is illustrated in Figure 4.3.9. The design was first tuned and optimized using the linear circuit simulator. The s-parameters of the tuned preliminary design are plotted in Figure 4.3.10. Once tuned, the layout was constructed, meshed, and simulated in Momentum. Circuit simulator and Momentum results compared favorably. Therefore, adjustments to the circuit model to account for parasitic effects were not required. Finally, a parameterized model was constructed in CST 
Microwave Studio and simulated using the time-domain solver. The results for each model are plotted in Figure 4.3.11. All three models compare favorably with a maximum discrepancy in insertion loss and phase difference of $0.08 \mathrm{~dB}$ and 0.5 degrees, respectively.

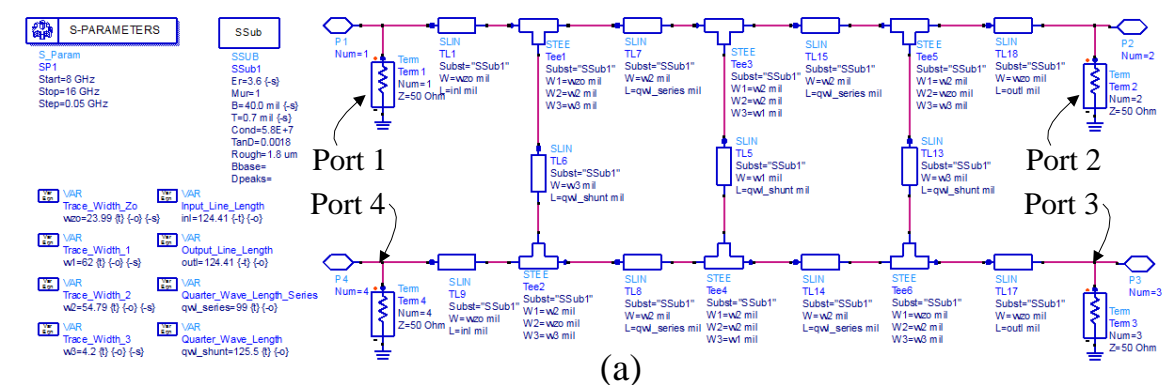

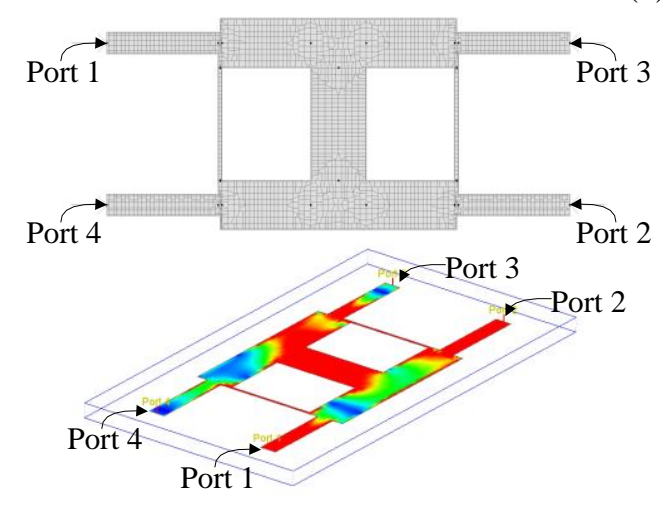

(b)

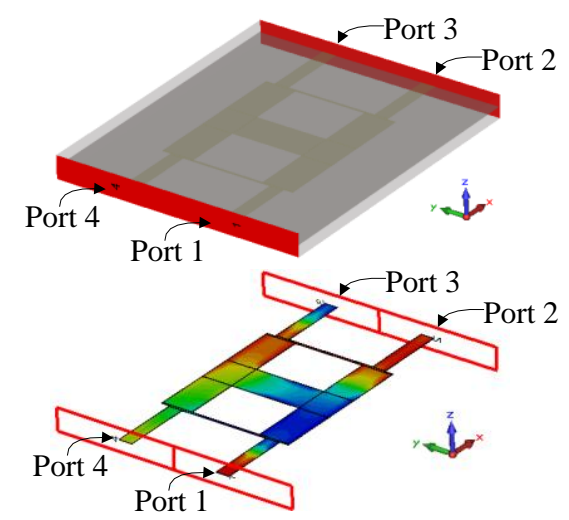

(c)

Figure 4.3.9 Three different branchline hybrid coupler models are constructed: (a) ADS schematic computed using a linear circuit simulator, (b) Momentum layout computed using the method of moments (MoM), and (c) Microwave Studio 3D model computed using the finite-difference time-domain (FDTD) method. 

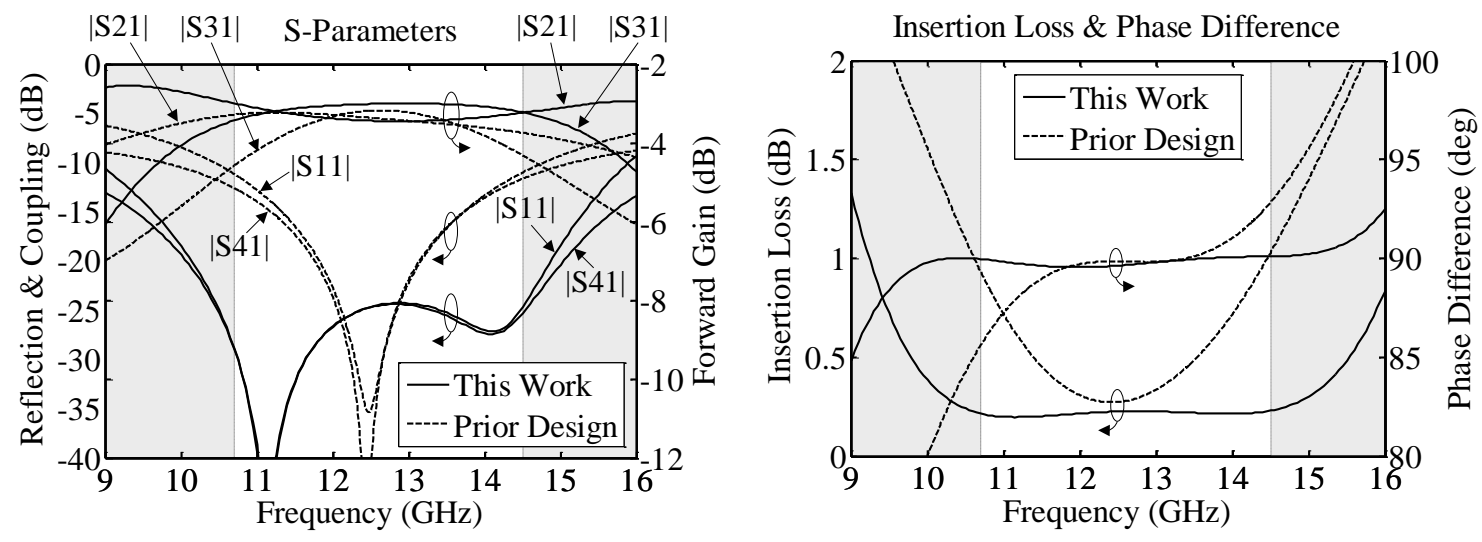

Figure 4.3.10 S-parameters of the preliminary dual-stage branchline hybrid coupler compared to the single-stage hybrid coupler of the prior design. The preliminary design exhibits return loss greater than $25 \mathrm{~dB}$, insertion loss lower than $0.3 \mathrm{~dB}$, RMS phase error of 0.188 degrees, and RMS amplitude error of $0.113 \mathrm{~dB}$.

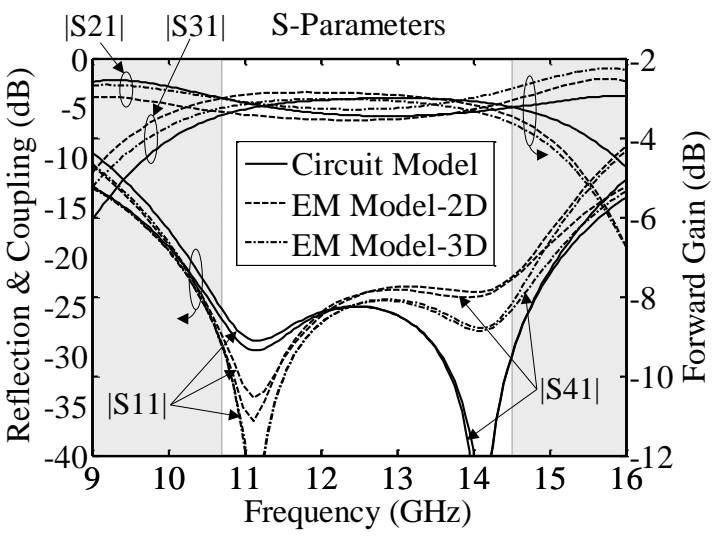

(a)

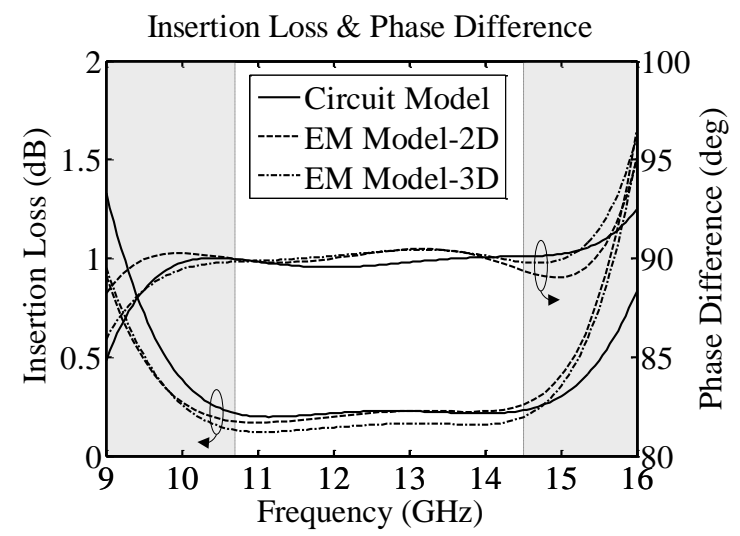

(b)

Figure 4.3.11 Comparison of branchline hybrid coupler s-parameters computed using three different simulation tools: circuit model in ADS, 2D layout model in Momentum, and 3D model in CST Microwave Studio. All three simulations compare favorably. All three models are illustrated in Figure 4.3.9. 


\subsubsection{Manufacturability Comparison}

To successfully implement a high frequency design using PCB materials and fabrication techniques, one must rigorously consider the impacts of material and manufacturing process tolerance on performance. Some designs reported in Literature neglect to consider this important element of the design process. This analysis is particularly important when comparing multiple design candidates as will be demonstrated in this section.

PCB materials are generally classified as substrates, prepregs, bond plies, metallic cladding, or resistive films. A set of electrical, mechanical, and thermal properties are supplied for each material by the manufacturer. The procedures required to measure these properties are standardized by the trade association known as the IPC or Association Connecting Electronics Industries. These standards are used by the electronics manufacturing industry and are accredited by the American National Standards Institute (ANSI).

Important properties of interest to a microwave designer include dielectric constant, dielectric loss tangent, standard thicknesses, and linear coefficients of thermal expansion. Metallic cladding surface roughness and finished thickness are also important for accurate modeling of transmission line loss and impedance. Each property is assigned a nominal value and a tolerance, and materials are screened to ensure compliance to published tolerances.

To perform a tolerance study using an electromagnetic design tool, model dimensions and material property values are defined as variables. Each variable is assigned a nominal value and a statistical distribution or tolerance. In this analysis, all substrate, prepreg, bond ply, and copper cladding materials are assigned properties with a uniform distribution across the range established by the nominal value and tolerance.

In addition to materials variance, the PCB process imposes limitations and contributes variance to the design. The three primary sources of variance are lamination registration, trace dimensions and plating anomalies, and via drill hole misalignment. Misregistration during a 
lamination causes some layers in the stackup to laterally shift. This is not a problem for stripline transmission lines which are insensitive to a shifted ground plane, but it is a problem for antenna structures and transitions between layers. For example, the capacitive feed in the prior design is very sensitive to a misalignments of only 2-3 mils, whereas the stripline aperture-coupled feed in the proposed design is much less sensitive to this misalignment. Transitions between layers require holes in ground planes and plated via drill holes which pierce through pads on each layer. When layers shift and drill holes are misaligned, the performance of these transitions quickly degrades. This is another advantage of the aperture-coupled feed which does not require vertical via transitions to antenna feeds in the antenna region; all transitions and feed lines are shielded by the ground plane. In this analysis, drill patterns and layers were assigned lateral offsets to replicate misregistration errors, and all trace widths were allowed to vary according to published capabilities of a typical PCB manufacturer [80].

A Monte Carlo simulation engine within the software simultaneously varies these properties, updates the 3D model, and computes s-parameters and far-field patterns. A significant amount of time and care are required to ensure a model is fully parameterized and can be modified by an automated simulation engine. This work employs CST Microwave Studio for this tolerance study of each antenna design. Unlike other simulators, Microwave Studio does not contain a Monte Carlo simulation engine. As a workaround, the optimization engine has been configured to functionally emulate a Monte Carlo simulation. Instructing the optimizer to utilize a genetic algorithm with a large population, high mutation rate, and unachievable goal functions approximates a uniformly-distributed random selection of property values similar to a Monte Carlo simulation.

Model properties and tolerances included in the preliminary design analysis are provided in Table 4.3.2, and manufacturing tolerances are provided in Table 4.3.3. 
Table 4.3.2 Material properties, dimensions, tolerances, and descriptions for all materials included in the Monte Carlo analysis for the preliminary antenna design. Tolerances are derived from Rogers Corporation's High Frequency Materials Product Selection Guide [81]. Model parameters are defined in Figure 4.3.2 and Figure 4.3.3.

\begin{tabular}{|c|c|c|c|c|c|}
\hline Sym. & Value & Tol. & Units & Material & Description \\
\hline$\varepsilon_{s t}$ & 1.96 & \pm 0.04 & - & Rogers RT/duroid® 5880LZ & Top Patch Substrate Die. Const. \\
\hline$\varepsilon_{p t}$ & 2.28 & \pm 0.04 & - & Rogers RO3001 ${ }^{\mathrm{TM}}$ & Top Patch Bold Film Die. Const. \\
\hline$\varepsilon_{s b}$ & 1.96 & \pm 0.04 & - & Rogers RT/duroid® $5880 \mathrm{LZ}$ & Bottom Patch Substrate Die. Const. \\
\hline$\varepsilon_{p b}$ & 3.00 & \pm 0.04 & - & Rogers RO3003 ${ }^{\mathrm{TM}}$ & Bottom Patch Bond Ply Die. Const. \\
\hline$\varepsilon_{f s t}$ & 3.00 & \pm 0.04 & - & Rogers RO3003 & Top Feed Substrate Die. Const. \\
\hline$\varepsilon_{f p t}$ & 3.00 & \pm 0.04 & - & Rogers RO3003 & Top Feed Bond Ply Die. Const. \\
\hline$\varepsilon_{f s m}$ & 3.00 & \pm 0.04 & - & Rogers RO3003 & Middle Feed Substrate Die. Const. \\
\hline$\varepsilon_{f p b}$ & 3.00 & \pm 0.04 & - & Rogers RO3003 & Bottom Feed Bond Ply Die. Const. \\
\hline$\varepsilon_{f s b}$ & 1.96 & \pm 0.04 & - & Rogers RT/duroid $\circledast 5880 \mathrm{LZ}$ & Bottom Feed Substrate Die. Const. \\
\hline$\varepsilon_{\text {hst }}$ & 3.50 & \pm 0.05 & - & Rogers RO3035 & Top Hybrid Substrate Die. Const. \\
\hline$\varepsilon_{h p t}$ & 2.90 & \pm 0.02 & - & Rogers ULTRALAM ${ }^{\circledR} 3908$ & Top Hybrid Prepreg Die. Const. \\
\hline$\varepsilon_{h s b}$ & 3.50 & \pm 0.05 & - & Rogers RO3035 & Bottom Hybrid Substrate Die. Const. \\
\hline ptst & 80 & \pm 4.0 & mil & Rogers RT/duroid® 5880LZ & Top Patch Substrate Thickness \\
\hline ptpt & 1.5 & \pm 0.15 & mil & Rogers RO3001 & Top Patch Bold Film Thickness \\
\hline pbst & 80 & \pm 4.0 & mil & Rogers RT/duroid® 5880LZ & Bottom Patch Substrate Thickness \\
\hline pbpt & 15 & \pm 1.5 & mil & Rogers RO3003 & Bottom Patch Bond Ply Thickness \\
\hline ftst & 10 & \pm 0.7 & mil & Rogers RO3003 & Top Feed Substrate Thickness \\
\hline ftpt & 10 & \pm 1.0 & mil & Rogers RO3003 & Top Feed Bond Ply Thickness \\
\hline fmst & 5 & \pm 0.5 & mil & Rogers RO3003 & Middle Feed Substrate Thickness \\
\hline fbpt & 10 & \pm 0.7 & mil & Rogers RO3003 ${ }^{\mathrm{TM}}$ & Bottom Feed Bond Ply Thickness \\
\hline fbst & 40 & \pm 2.0 & mil & Rogers RT/duroid® 5880LZ & Bottom Feed Substrate Thickness \\
\hline bcpt & 1.0 & \pm 0.13 & mil & Rogers ULTRALAM® 3908 & Hybrid Top Bond Film Thickness \\
\hline bcst & 20 & \pm 1.0 & mil & Rogers RO3035 & Hybrid Substrate Thickness \\
\hline bcpb & 1.0 & \pm 0.13 & mil & Rogers ULTRALAM® 3908 & Hybrid Top Bond Film Thickness \\
\hline bcsb & 20 & \pm 1.0 & mil & Rogers RO3035 & Hybrid Bottom Substrate Thickness \\
\hline tcu & 0.71 & \pm 0.2 & mil & Rolled Copper Cladding & All Copper Layers \\
\hline
\end{tabular}


Table 4.3.3 Manufacturing process tolerances are applied to model dimensions in the Monte Carlo analysis for the preliminary antenna design. All tolerances are derived from process capabilities of modern PCB manufacturers.

\begin{tabular}{lllll}
\hline \multicolumn{5}{l}{ Manufacturing Process Tolerances } \\
\hline Sym. & Value & Tol. & Units & Description \\
\hline ptx & 203.0 & \pm 0.5 & mil & Top Patch Length in X-Direction \\
pty & 203.0 & \pm 0.5 & mil & Top Patch Length in Y-Direction \\
pbx & 215.9 & \pm 0.5 & mil & Bottom Patch Length in X-Direction \\
pby & 215.9 & \pm 0.5 & mil & Bottom Patch Length in Y-Direction \\
tfaw & 7.56 & \pm 0.5 & mil & Top Feed Arm Width \\
tfw & 42.2 & \pm 0.5 & mil & Top Feed Width \\
bfaw & 8.08 & \pm 0.5 & mil & Bottom Feed Arm Width \\
bfw & 42.4 & \pm 0.5 & mil & Bottom Feed Width \\
gsw & 39.6 & \pm 0.5 & mil & Ground Slot Width \\
gssw & 28.0 & \pm 0.5 & mil & Ground Slot Stub Width \\
re1x & 0.00 & \pm 3.5 & mil & Layers 1 and 2 Offset Registration in X-Direction \\
re1y & 0.00 & \pm 3.5 & mil & Layers 1 and 2 Offset Registration in Y-Direction \\
re2x & 0.00 & \pm 3.5 & mil & Layers 4 and 5 Offset Registration in X-Direction \\
re2y & 0.00 & \pm 3.5 & mil & Layers 4 and 5 Offset Registration in Y-Direction \\
vfr & 12.5 & \pm 1.5 & mil & Via Fence Radius \\
\hline
\end{tabular}

The Monte Carlo investigation is divided into two analyses. The first analysis includes only material property tolerances given in Table 4.3.2, and the second includes both the materials tolerances in Table 4.3.2 and manufacturing process tolerances in Table 4.3.3. The first analysis consists of 50 simulation runs, and the second includes an additional 50 simulation runs. Each simulation run requires roughly 40 minutes on a quad-core laptop computer (c. 2010). The performance curves for port s-parameters, co-pol and cross-pol gain, and radiation pattern theta cuts are provided in Figure 4.3.12, Figure 4.3.13, and Figure 4.3.14, respectively. The captions summarize results and compare the two designs. 

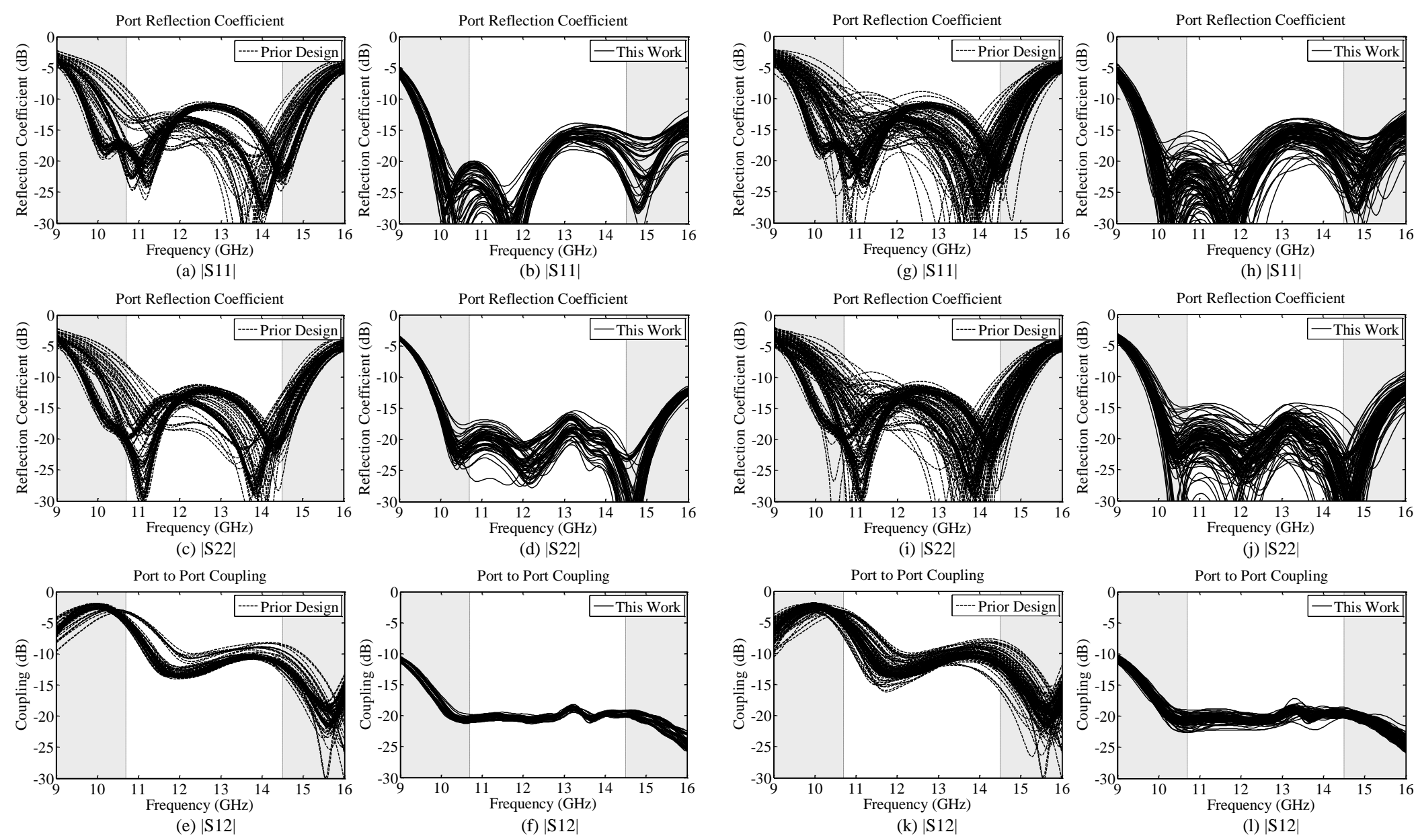

Figure 4.3.12 Monte Carlo analysis results comparison for the proposed and prior antenna designs for (a-f) 50 simulation runs varying material properties only and (g-1) 50 additional simulation runs varying material properties and applying manufacturing process tolerances. The top, middle, and bottom rows represent $|\mathrm{S} 11|,|\mathrm{S} 22|$, and $|\mathrm{S} 12|$, respectively. Although both designs are reasonably stable, the proposed design exhibits less variation and overall better performance. 


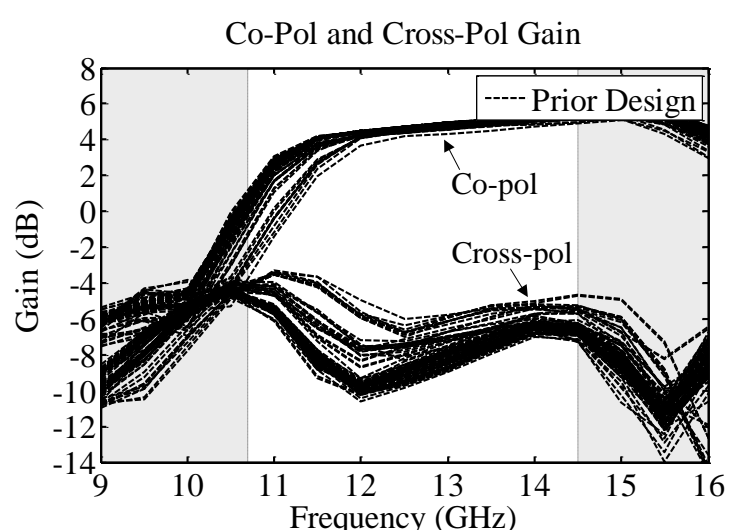

(a)

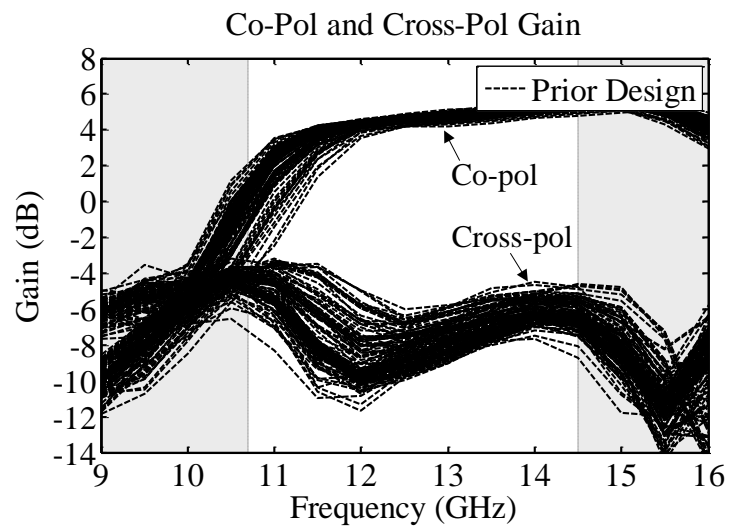

(c)

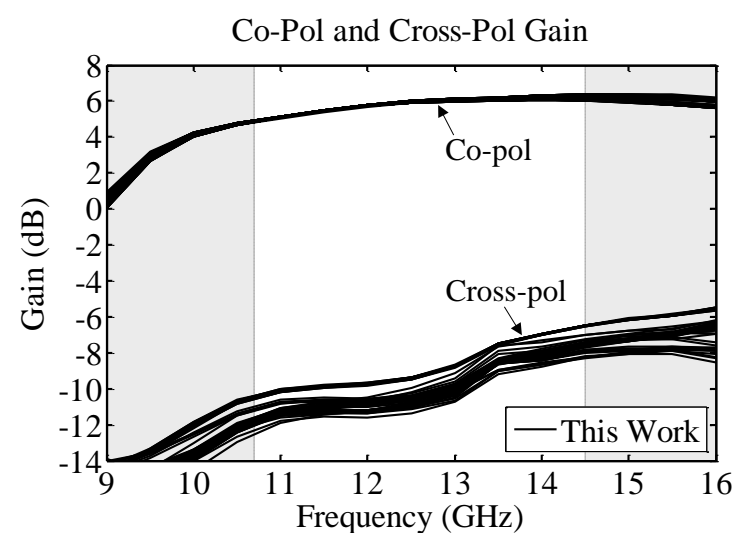

(b)

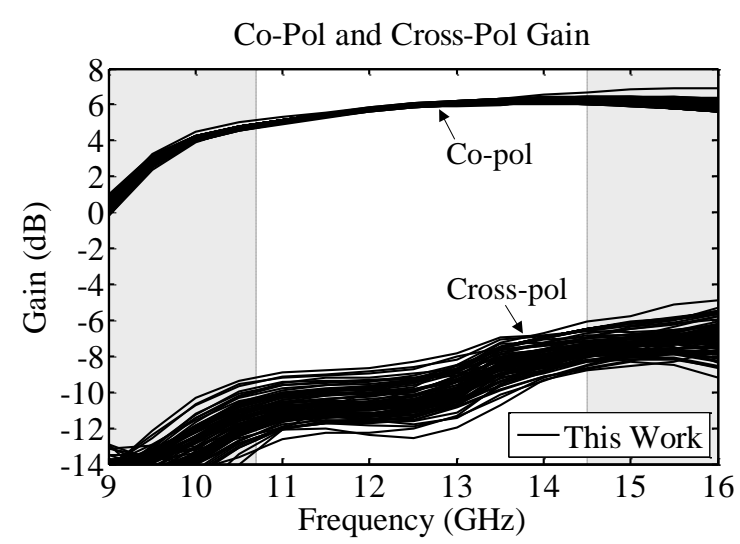

(d)

Figure 4.3.13 Monte Carlo analysis results comparison for the proposed and prior antenna designs for (a-b) 50 simulation runs varying material properties only and (c-d) 50 additional simulation runs varying material properties and applying manufacturing process tolerances. The left column represents the prior design, and the right column represents the proposed design. Co-pol gain for the prior design varies dramatically below $12 \mathrm{GHz}$. Co-pol gain for the proposed design is stable with a peak-to-peak variation of $0.6 \mathrm{~dB}$. Cross-pol gain variation is less than $4 \mathrm{~dB}$ for the proposed design compared to $6 \mathrm{~dB}$ for the prior design. 

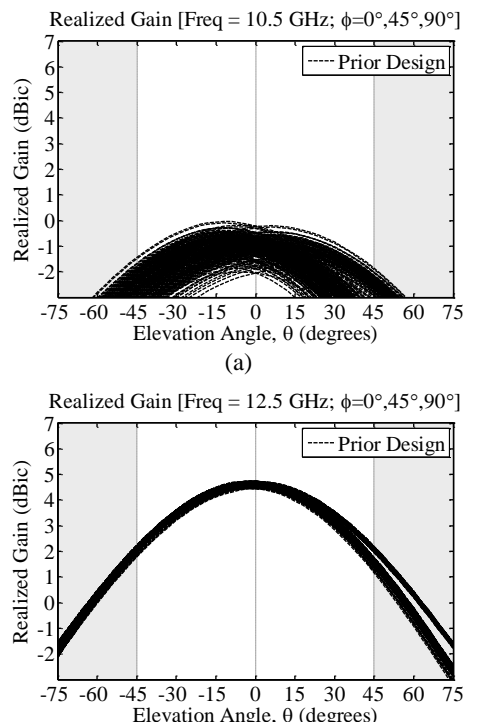

(c)

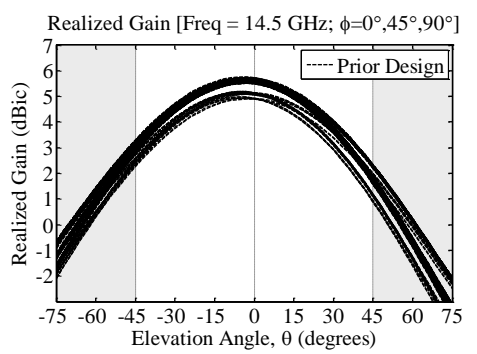

(e)



(b)

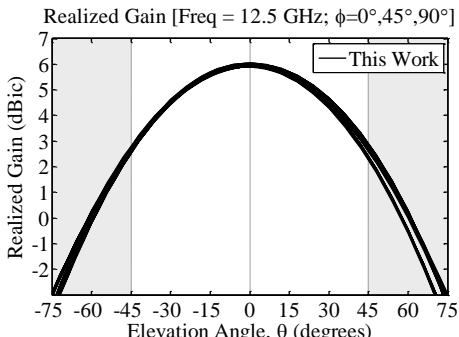

(d)

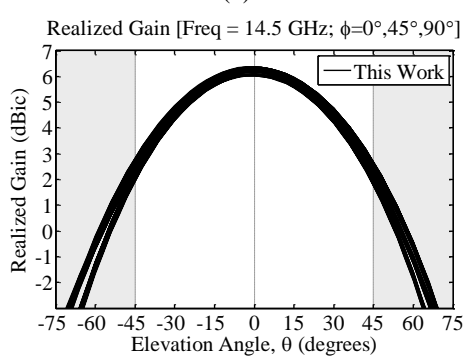

(f)



(g)

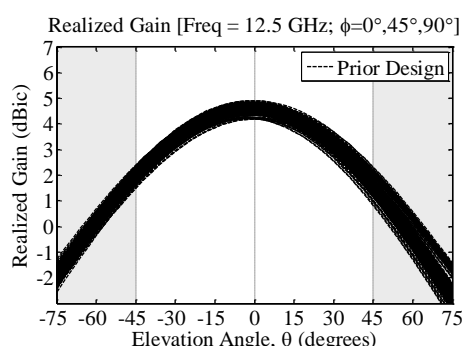

(i)

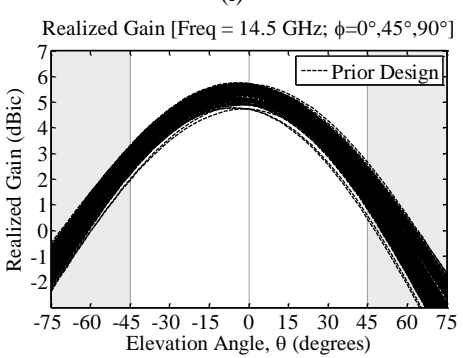

(k)

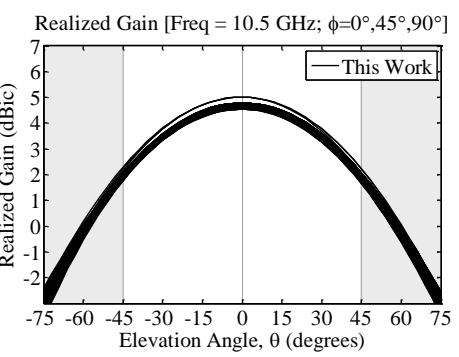

(h)



(j)

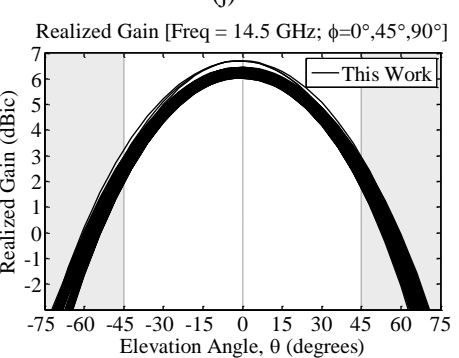

(1)

Figure 4.3.14 Monte Carlo analysis results comparison for the proposed and prior antenna designs for (a-f) 50 simulation runs varying material properties only and (g-l) 50 additional simulation runs varying material properties and applying manufacturing process tolerances. The top, middle, and bottom rows correspond to $10.5,12.5$, and $14.5 \mathrm{GHz}$, respectively. Theta cuts for $\phi=0^{\circ}, 45^{\circ}, 90^{\circ}$ are included on each plot, so the spread is related to radiation pattern uniformity. The proposed design is less sensitive to tolerances and overcomes the non-symmetry and lower peak gain exhibited by the prior design. 


\subsection{Final Design}

\subsubsection{Antenna Topology}

Two different layouts were considered for the final design. Initially, a layout which maintained the same orientation of the patches and feeds as the preliminary design was selected. After some thought and modeling, it was determined that rotating the patches, feed slot, and feeds by 45 degrees reduces insertion loss of the antenna feeds and also leads to a symmetric hybrid. The two designs are illustrated in Figure 4.4.1 and described in the caption.

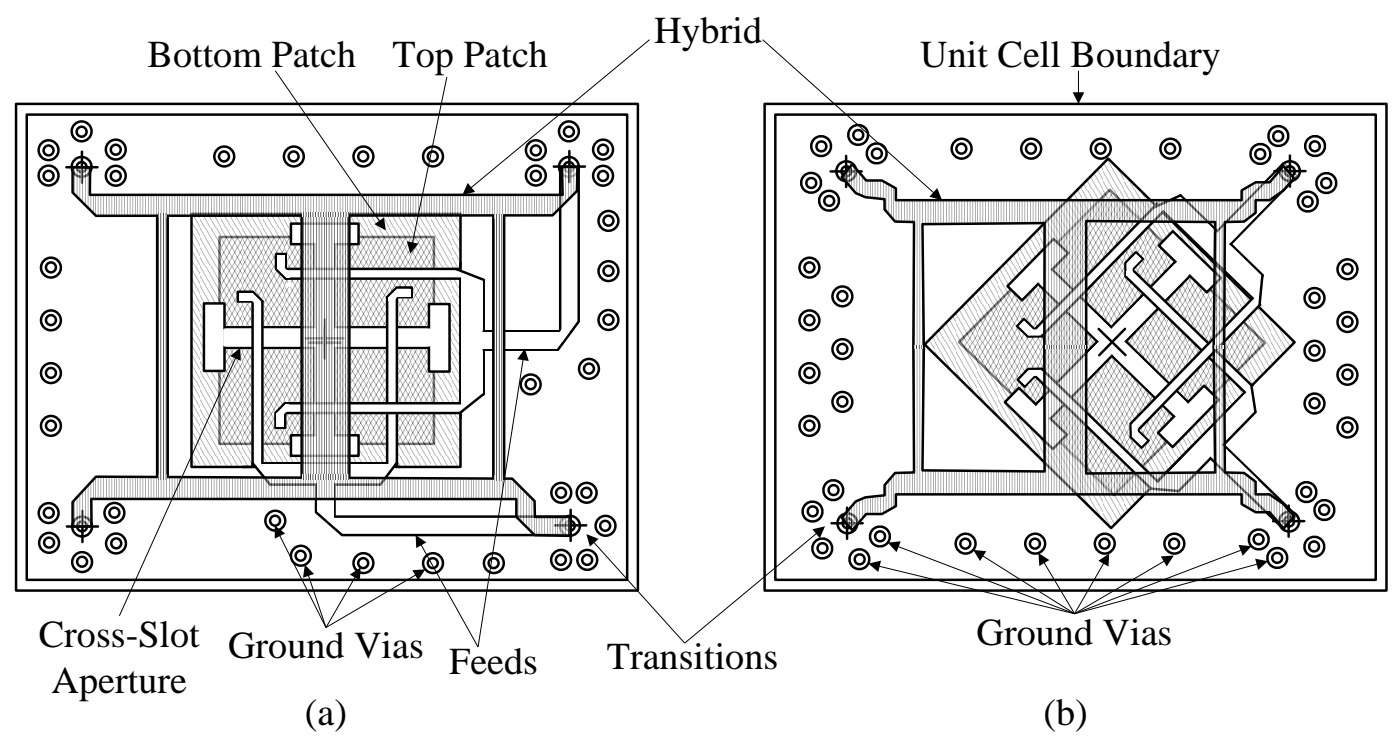

Figure 4.4.1 Two candidate final design concepts. Design (a) is very similar to the preliminary design, except the hybrid is connected to stripline antenna feeds through a 5-via transition. Notice the non-symmetry of the hybrid design. This slightly degrades hybrid performance. Design (b) eliminates bends and some length from the stripline feeds and allows for a symmetric hybrid structure. For each design, the antenna and hybrid were optimized. Design (b) was selected due to overall superior performance.

The final design requires antenna-to-hybrid transition vias, via pads, backdrills, and backfills.

Via transitions between the hybrid and antenna and must be impedance controlled. The transition consists of a center signal via surrounded by five ground vias, similar to the popular five-via transition found in microwave design guides [82]. When the transition via is manufactured, the 
laminated portion of the stackup is drilled and plated. This drill hole spans the entire thickness of the laminated portion of the stackup. For a signal via that connects two internal layers, two via stubs on top and bottom of the interlayer section are created. The desired portion is the interlayer section that connects the two desired layers. The stubs on top and bottom contribute parasitic capacitance and degrade the wideband performance of the transition.

One method to eliminate stubs is to employ a process known as backdrilling. In this process, the manufacturer removes unwanted via stubs by drilling holes using a bit (or laser) that has a slightly larger diameter than the plated via as illustrated in Figure 4.4.2. This process reduces the length of each unwanted stub to 1-2 mil and consequently reduces parasitic capacitance and improves transition performance. The photographs in Figure 4.4.3 further illustrate the features of a backdrilled via.

After backdrilling all unwanted stubs, the manufacturer fills these stubs with dielectric material in a process known as backfilling. There are several backfill materials with different dielectric properties, so one can select the material that most closely matches the dielectric properties of the substrates that were drilled. To improve simulation accuracy, these features are incorporated into the 3D electromagnetic model as pictured in Figure 4.4.4.

Backdrilling many vias in this way increases PCB manufacturing cost. As an alternative, the designer may choose to compensate for known parasitic effects contributed by the via stubs in the circuit design, but this approach further complicates a compact microwave design and limits the bandwidth of the transition. This approach is favored for narrowband applications where cost is a primary driver. 


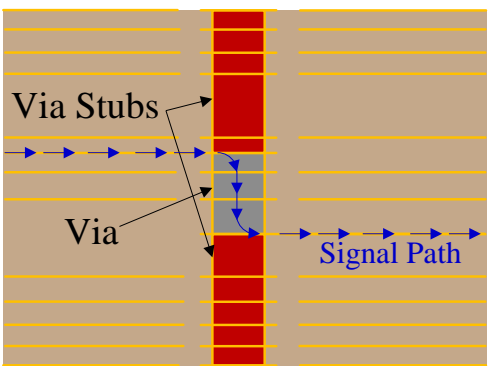

(a)

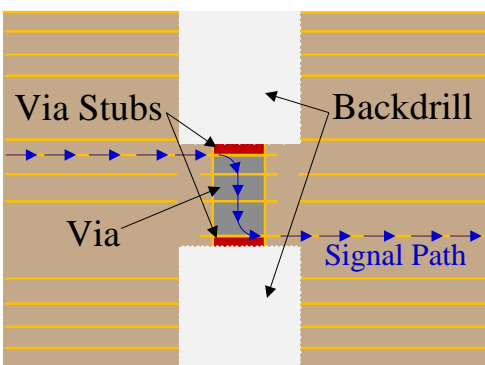

(b)

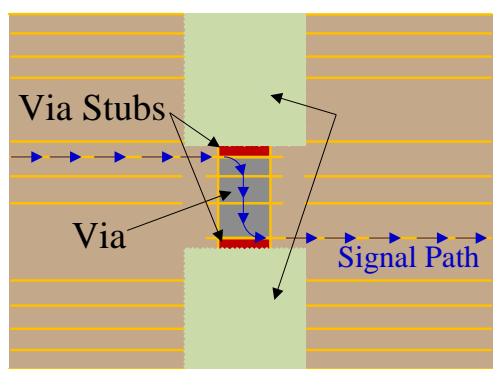

(c)

Figure 4.4.2 Conceptual overview of the backdrill and backfill processes used to eliminate unwanted via stubs from high frequency interlayer transitions. The unwanted via stubs (red) in (a) are removed in the backdrill process in (b) and are filled with backfill material (green) in (c). The remaining via consists of the desired interlayer section as well as two 1-2 mil via stubs.

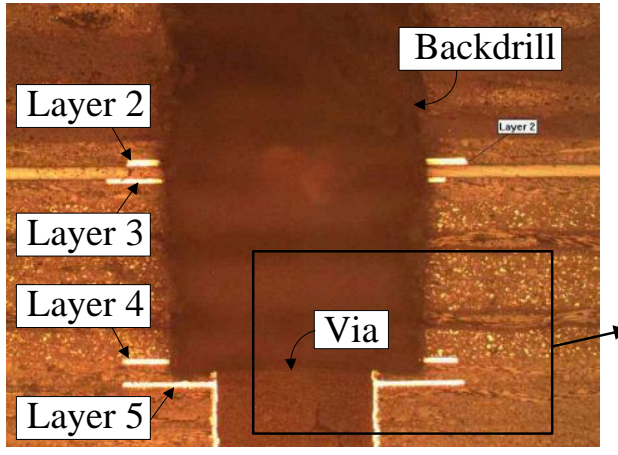

(a)

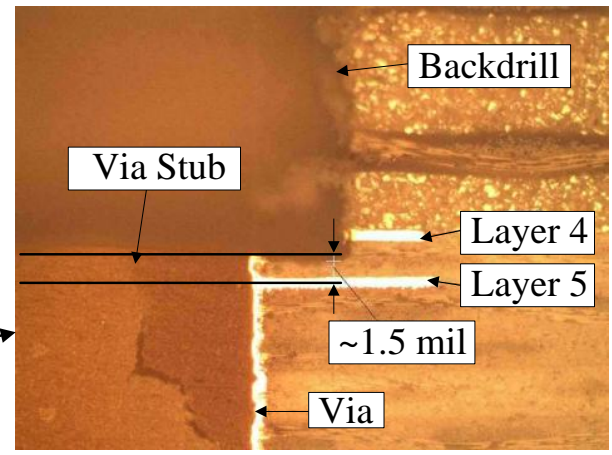

(b)

Figure 4.4.3 Photographs taken with a digital microscope of a backdrilled via cross-section from the prior antenna design illustrate (a) the backdrilled region with 4 metal layers in view and (b) the interface between the via and backdrilled region. Via stub length reduces from $60 \mathrm{mil}$ to $1.5 \mathrm{mil}$. The photograph in (a) also illustrates a 3-4 mil misalignment between layers 2 and 3. This via is also pictured in the x-ray image and labeled "transition to coupler" in Figure 4.2.3. For reference, the via diameter is nominally 20 mil.

The preliminary design does not comply to the port-to-port isolation and cross-polarization level requirements, so a study was performed on the final design to regain compliance. Four approaches were considered. In [83], the author proposes a method to improve isolation by maximizing the separation between the tips of the stripline feeds. This is a novel approach and 
can be easily implemented. A second approach found in an antenna patent [84] utilizes a dualpath stripline fork (i.e. reactive divider) for one feed and a conventional single-path stripline for the second feed, essentially minimizing feed overlap. This approach achieves very high isolation between ports, but requires significant rework to the model, and is therefore excluded from further consideration. A third approach considered is to increase the feed substrate thickness to further separate the two feeds. However, this method is limited by available substrate thicknesses. It was determined by simulation that the increase cannot be more than 6-8 mil before the coupling to the slot in the ground plane weakens. The final approach was attempted after it was noticed that a small fraction of the energy was guided directly from one feed transition to the orthogonal feed. This may indicate the excitation of an evanescent mode that can occur at discontinuities in the waveguide structure [48] or a parallel plate mode which can often plague PCB-based phased arrays with scan blindness [72]. One method to suppress a parallel plate mode in a stripline structure is to add what are known as fencing vias or ground vias on either side of the stripline which stitch together the top and bottom ground planes.

After assessing feasibility of each approach and performing preliminary simulations of modified feed designs, it was determined that by bending the tips of the open-circuit stripline stubs at the end of each feed and inserting fencing vias between feed ports, the isolation and cross-polarization level gain compliance. These design modifications are highlighted in Figure 4.4.5.

\subsubsection{Hybrid Topology}

Two hybrid designs corresponding to the two topologies presented in Figure 4.4.1 were constructed. The symmetric design performs well and easily integrates with the favored unit cell topology. The non-symmetric design proved more complicated to optimize and exhibited inferior performance. Therefore, the symmetric design was selected and further optimized. 
Ground Layers

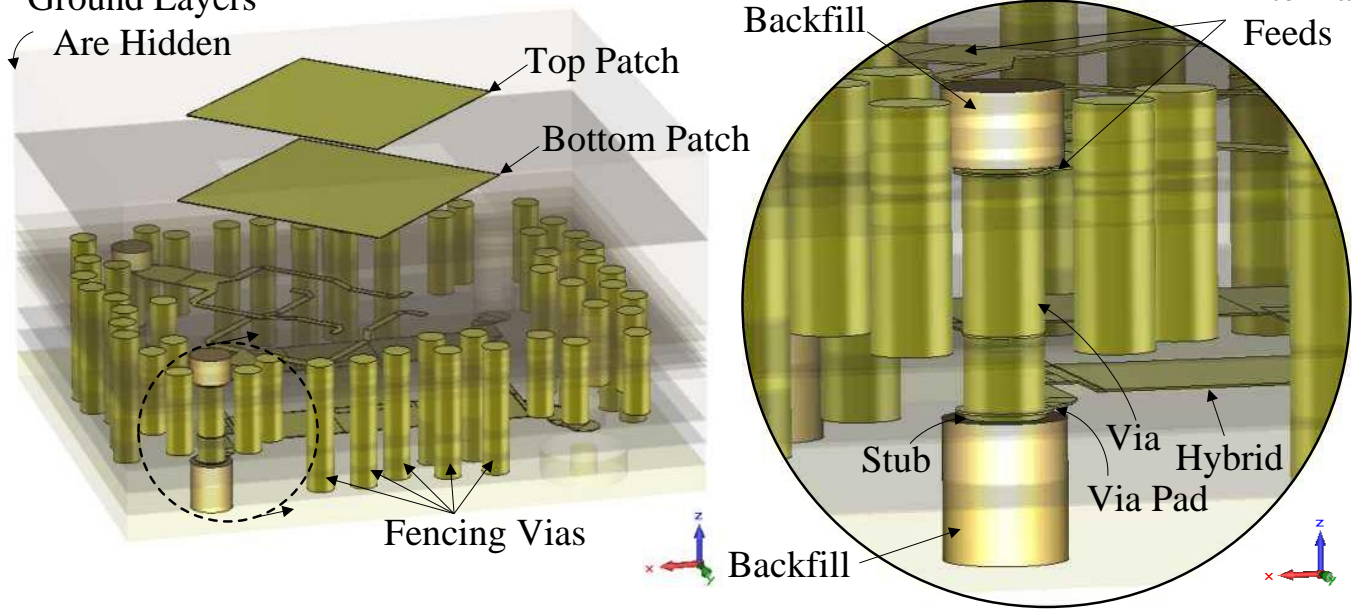

Figure 4.4.4 The hybrid to antenna transition consists of a quasi-coaxial structure with a center via and five surrounding ground vias. To eliminate parasitic capacitance, the remaining via stubs on top and bottom are backdrilled and backfilled with a material that has similar properties to the substrates.

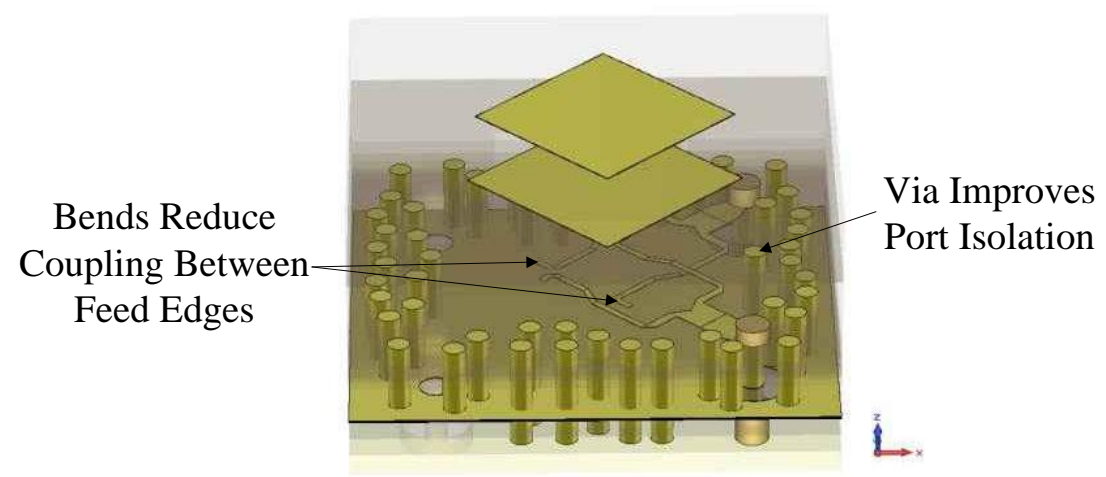

Figure 4.4.5 Port-to-port isolation improves from $17 \mathrm{~dB}$ to $22 \mathrm{~dB}$ and crosspolarization level improves from $-15 \mathrm{~dB}$ to $-17 \mathrm{~dB}$ by adding a ground via between ports and maximizing the distance between the tips of the feed forks. 


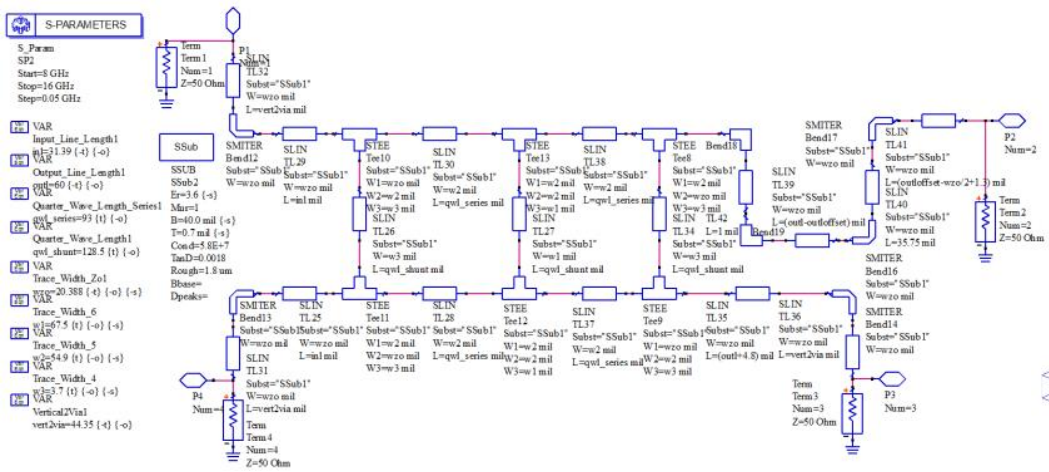

(a)

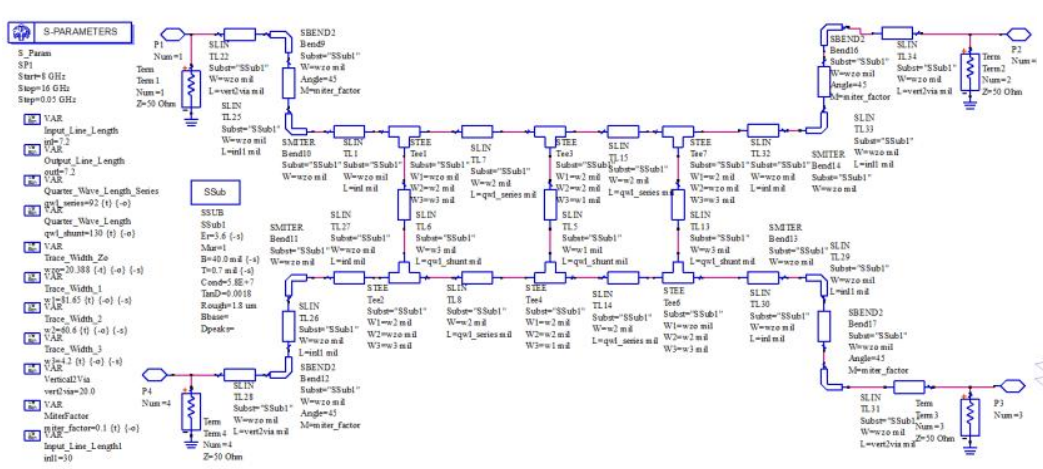

(d)

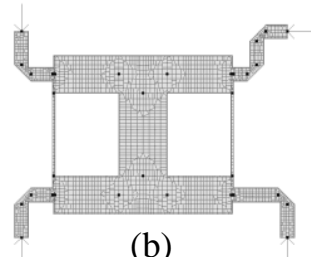

(b)

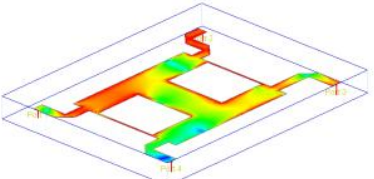

(c)

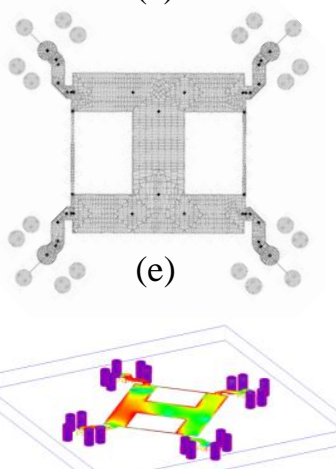

(f)

Figure 4.4.6 ADS models for two hybrid designs corresponding to the two topologies presented in Figure 4.4.1. The schematics are pictured in (a) and (d), meshed layouts in (b) and (e), and surface currents in (c) and (f). The second design in (d-f) exhibits best performance. 


\subsubsection{Antenna Performance}

The design changes discussed in the prior section were incorporated, and the design was optimized in Microwave Studio. Performance is summarized in Table 4.4.1. Updated performance curves are presented and described in Figure 4.4.7, Figure 4.4.8, Figure 4.4.9, and Figure 4.4.10.

Table 4.4.1 Performance comparison of final, preliminary, and prior antenna and hybrid designs compared with the requirements for each design. Nominal values for material properties and model dimensions are assumed.

\begin{tabular}{|c|c|c|c|c|c|c|}
\hline Antenna Parameter & $</>$ & Req. & $\begin{array}{l}\text { Prior } \\
\text { Design }\end{array}$ & $\begin{array}{l}\text { Prelim. } \\
\text { Design }\end{array}$ & $\begin{array}{c}\text { Final } \\
\text { Design }\end{array}$ & Units \\
\hline Min. Operational Frequency & $=$ & 10.7 & 10.7 & 10.7 & 10.7 & $\mathrm{GHz}$ \\
\hline Max. Operational Frequency & $=$ & 14.5 & 14.5 & 14.5 & 14.5 & $\mathrm{GHz}$ \\
\hline Port Reflection Coefficient & $<$ & -10 & -13 & -16 & -11 & $\mathrm{~dB}$ \\
\hline Realized Gain, $10.7-12.7 \mathrm{GHz}$ & $>$ & 4.2 & 0.4 & 4.3 & 4.8 & dBic \\
\hline Realized Gain, $13.7-14.5 \mathrm{GHz}$ & $>$ & 5.5 & 4.9 & 6.0 & 7.0 & dBic \\
\hline Port-to-Port Coupling & $<$ & -20 & -5.0 & -17 & -22 & $\mathrm{~dB}$ \\
\hline Cross-Pol. Level & $<$ & -17 & -5.3 & -15 & -17 & $\mathrm{~dB}$ \\
\hline Unit Cell Size, X-dimension & $=$ & 541 & 603 & 541 & 541 & mil \\
\hline Unit Cell Size, Y-dimension & $=$ & 469 & 522 & 469 & 469 & mil \\
\hline Hybrid Parameter & $</>$ & Req. & $\begin{array}{c}\text { Prior } \\
\text { Design }\end{array}$ & $\begin{array}{l}\text { Prelim. } \\
\text { Design }\end{array}$ & $\begin{array}{c}\text { Final } \\
\text { Design }\end{array}$ & Units \\
\hline Min. Operational Frequency & $=$ & 10.7 & 10.7 & 10.7 & 10.7 & $\mathrm{GHz}$ \\
\hline Max. Operational Frequency & $=$ & 14.5 & 14.5 & 14.5 & 14.5 & $\mathrm{GHz}$ \\
\hline Port Reflection Coefficient & $<$ & -15 & -9.8 & -22 & -15 & $\mathrm{~dB}$ \\
\hline Port-to-Port Coupling & $<$ & -15 & -9.8 & -22 & -17 & $\mathrm{~dB}$ \\
\hline Insertion Loss & $<$ & 0.5 & 0.95 & 0.30 & 0.40 & $\mathrm{~dB}$ \\
\hline RMS Phase Error & $<$ & 0.8 & 1.402 & 0.188 & 0.628 & deg. \\
\hline RMS Amplitude Error & $<$ & 0.2 & 0.297 & 0.113 & 0.156 & $\mathrm{~dB}$ \\
\hline Unit Cell Size, X-dimension & $=$ & 541 & 603 & 541 & 541 & mil \\
\hline Unit Cell Size, Y-dimension & $=$ & 469 & 522 & 469 & 469 & mil \\
\hline
\end{tabular}




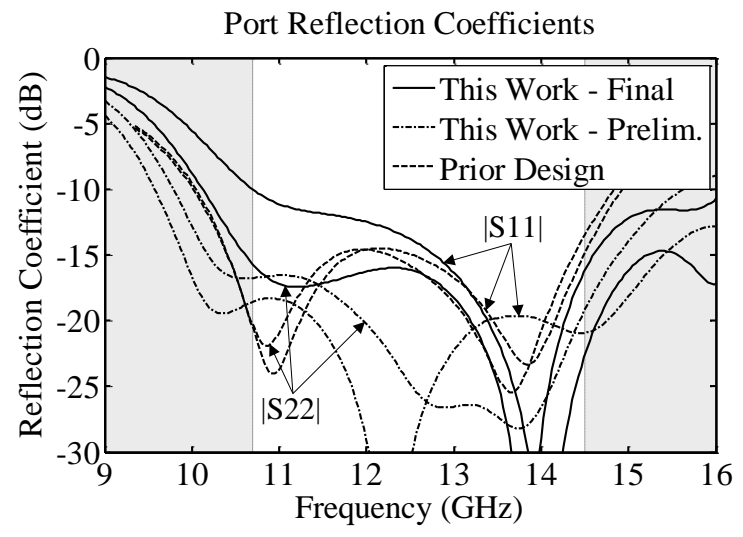

(a)

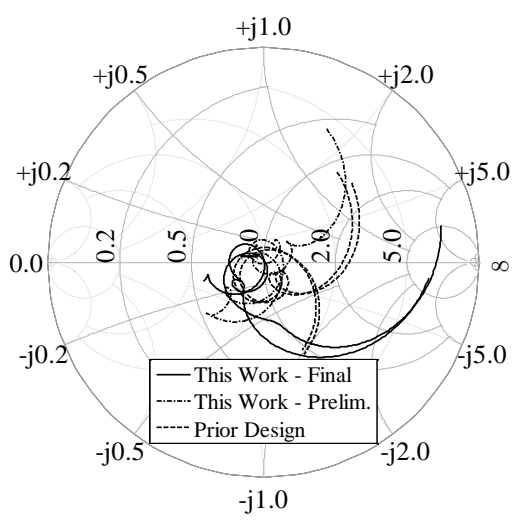

(b)

Figure 4.4.7 Comparison of port reflection coefficients for the final, preliminary, and prior designs. Reflection coefficients are plotted (a) in rectangular form and (b) on a Smith chart. The final design exhibits slightly degraded port 1 return loss in the lower portion of the band.
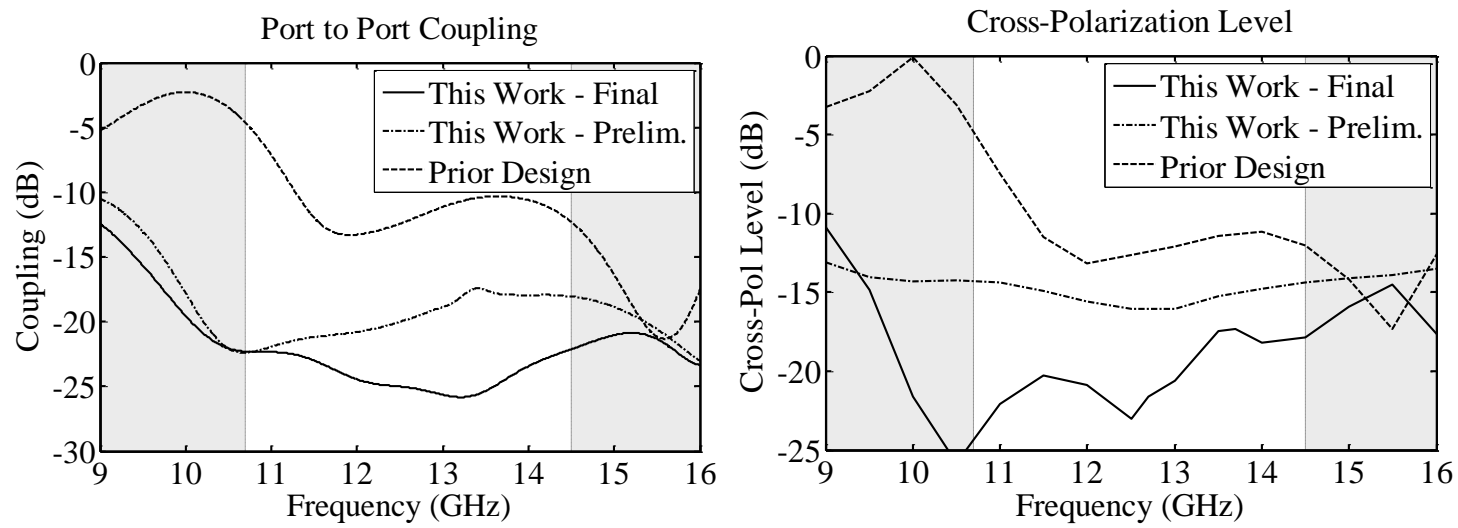

Figure 4.4.8 Comparison of port-to-port coupling and cross-polarization level of the final, preliminary, and prior designs. Both parameters have improved by an average of roughly $10 \mathrm{~dB}$ across the operating bandwidth. Coupling is less than $22 \mathrm{~dB}$, and cross-polarization level is less than $-17 \mathrm{~dB}$. 


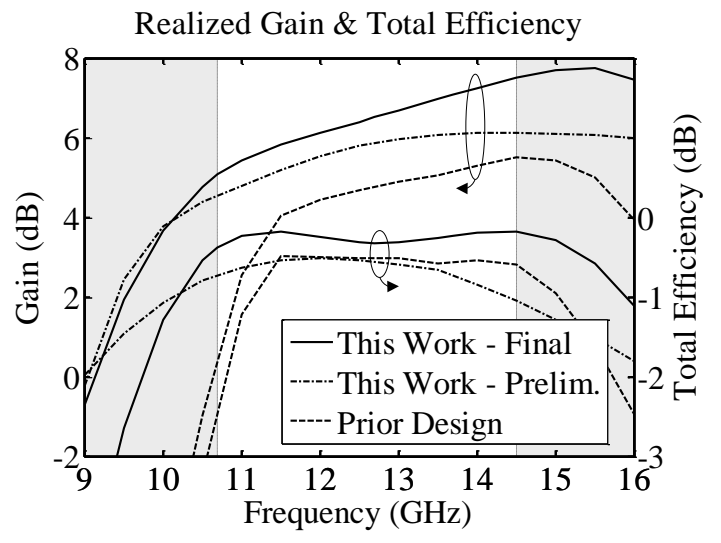

Figure 4.4.9 Comparison of gain and efficiency for the final, preliminary, and prior designs. The final design achieves an increase in gain of roughly $2 \mathrm{~dB}$ relative to the prior design. 


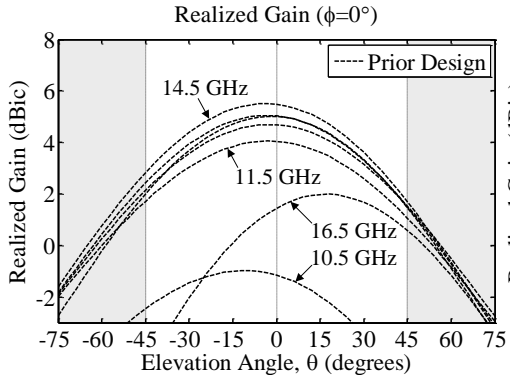

(a)

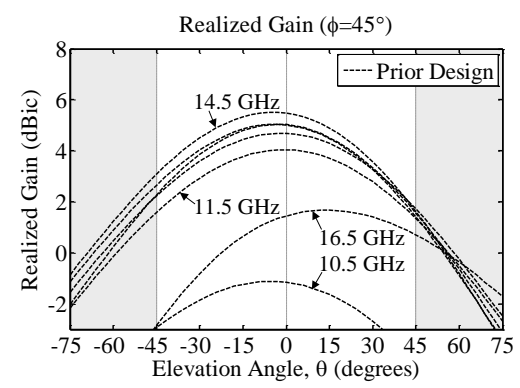

(d)

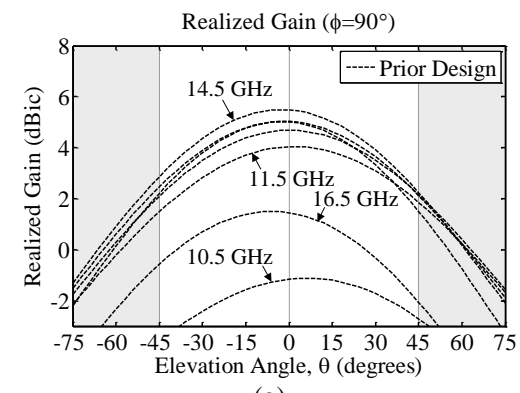

(g)

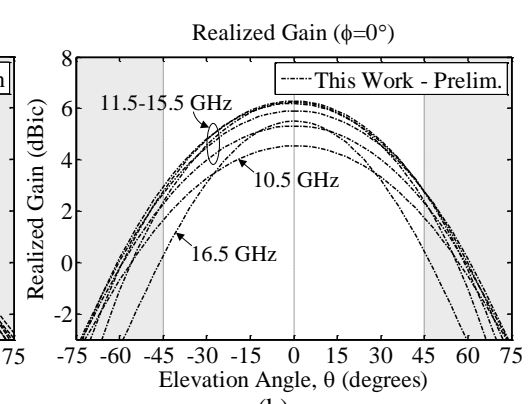

(b)

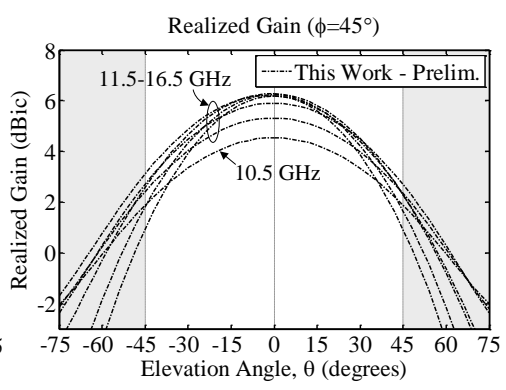

(e)

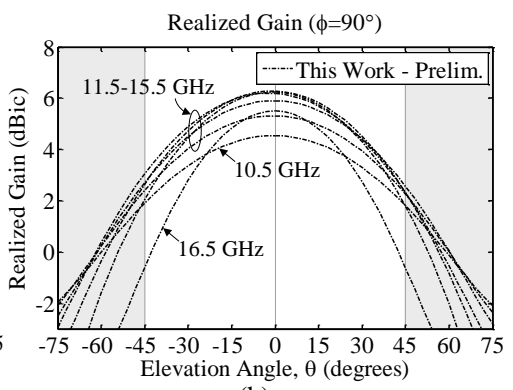

(h)

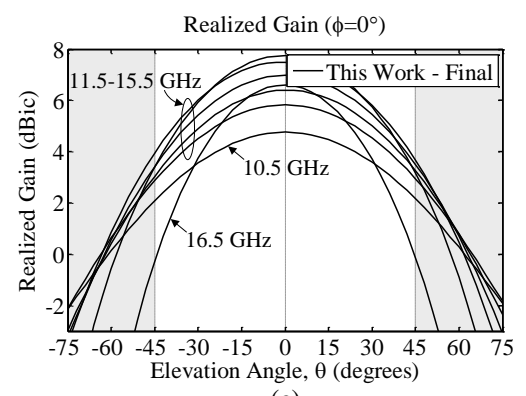

(c)



(f)

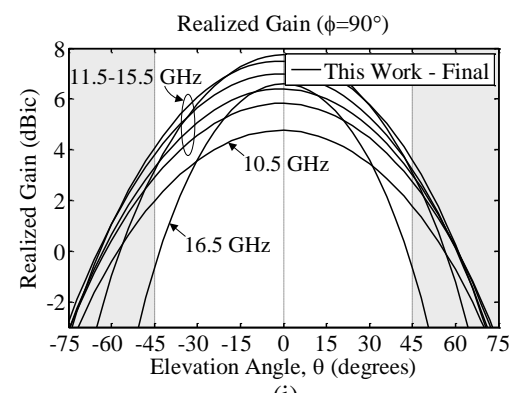

(i)

Figure 4.4.10 Comparison of 2D theta cuts of the 3D radiation patterns. Plots on the left, middle, and right columns correspond to the prior, preliminary, and final designs. The top, middle, and bottom rows correspond to $\phi=0^{\circ}, 45^{\circ}$, and $90^{\circ}$, respectively. Curves for seven frequencies are given on each plot, uniformly spaced from 10.5 to $16.5 \mathrm{GHz}$. The final design represents an improvement over the preliminary design in peak gain and gain uniformity.

\subsubsection{Hybrid Performance}

A comparison between the final and preliminary hybrid design is given in Figure 4.4.11. 


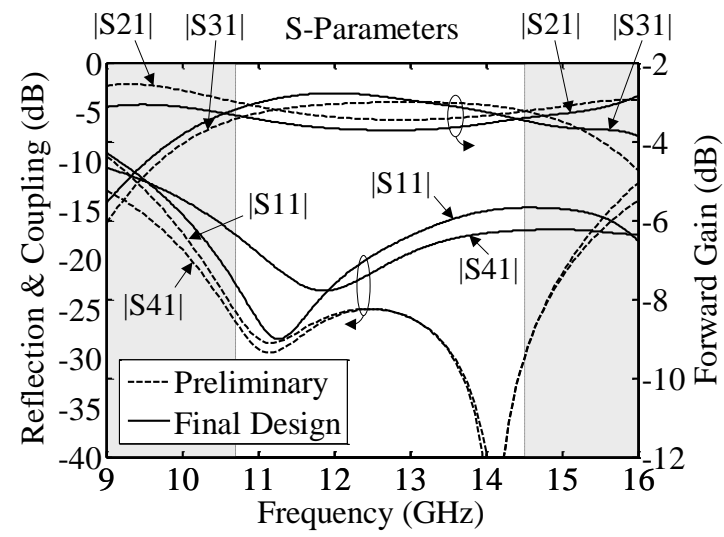

(a)

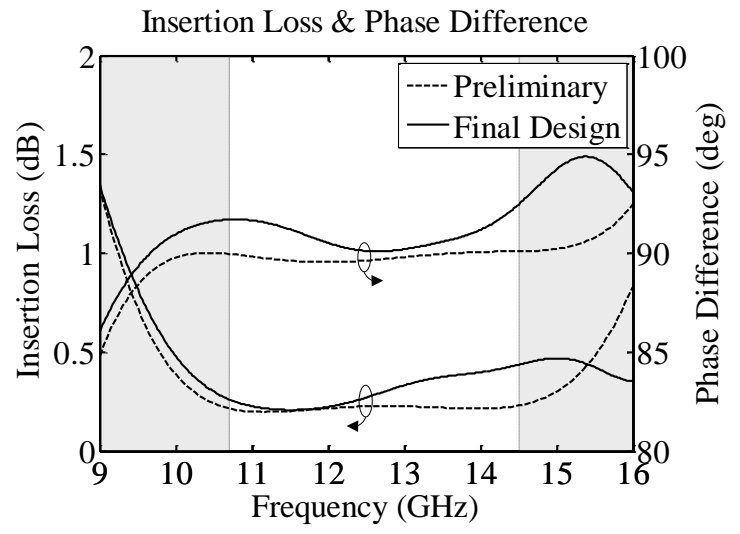

(b)

Figure 4.4.11 S-parameters of the preliminary and final hybrid designs computed in Microwave Studio. The final design has slightly degraded performance due to the addition of via transitions, but still meets requirements. Insertion loss is less than $0.4 \mathrm{~dB}$, RMS phase error is 0.628 degrees, and RMS amplitude error is $0.156 \mathrm{~dB}$.

\subsubsection{Cascaded Performance}

To gain simulation efficiency, the electromagnetic models for the antenna and hybrid were optimized separately in Microwave Studio, and s-parameters were cascaded in ADS. This is a commonly employed process in microwave design. A structure is typically dissected into separable portions which can be independently simulated and optimized. There are two reasons for dividing a problem in this way. The first relates to physical limitations of the processing machine. As the problem size increases, the number of mesh cells increases. As the number of mesh cells increases, the amount of random access memory required increases. Most modern machines (c. 2012) contain 4 or 8 GB of RAM and are therefore limited in the number of mesh cells that can be computed in a simulation run. For a large problem, (e.g. a small antenna installed on a large spacecraft) a significant amount of RAM is required (e.g. 128 GB). The second problem relates to simulation time. As the number of mesh cells increases by a factor of $N$, the number of computations, and hence the computation time, increases by factor of $N^{2}$. Therefore, structures are simulated separately to save time and computational resources. 
For added learning, both models were combined in Microwave Studio, and the integrated model was simulated. Combining models requires the removal of ports at the output of the hybrid and input to the antenna. The additional length added by those port launches were phase deembedded from the s-parameters of each separate model. A comparison between the separately simulated and combined models is given in Figure 4.4.12. The reflection coefficient curves compare favorably. There appears to be a small phase error that is likely caused by port parasitics that are present in each separate design, but absent in the combined design. The port-to-port coupling curves compare less favorably. The combined design exhibits increased coupling that is likely due to a small impedance mismatch at the interface between combined models. This mismatch causes some of the signal entering one hybrid input port to reflect back toward both hybrid input ports, thereby increasing port-to-port coupling.

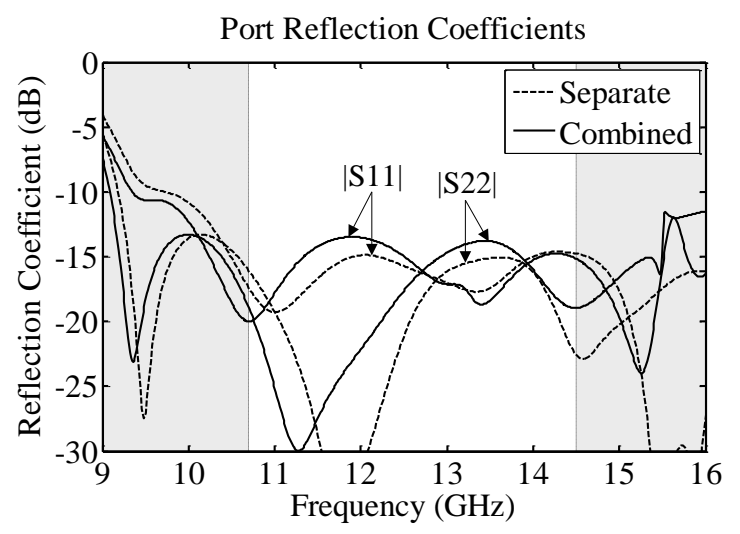

(a)



(b)

Figure 4.4.12 Two simulation approaches compare favorably. (a) A small phase error is observed in port reflection coefficients which appears as a horizontal shift between the two curves. The combined model exhibits roughly 2-4 dB higher coupling between ports.

\subsubsection{Array Performance}

To verify the performance in an array configuration, a 64-element array was constructed and simulated using Microwave Studio. The finite array model is pictured in Figure 4.4.13. Each port 
was simultaneously excited, and the resultant far-field array pattern was computed. The computed radiation pattern at $14 \mathrm{GHz}$ is provided in Figure 4.4.14. The array is expected to achieve a realized gain close to $22.6 \mathrm{dBi}$, assuming an element gain at $14 \mathrm{GHz}$ of $6.3 \mathrm{dBi}$ and an amplitude taper loss of roughly $1.8 \mathrm{~dB}$ for a $-22 \mathrm{~dB}$ maximum sidelobe level [44]. The computed realized gain compares favorably at $22.2 \mathrm{dBi}$.

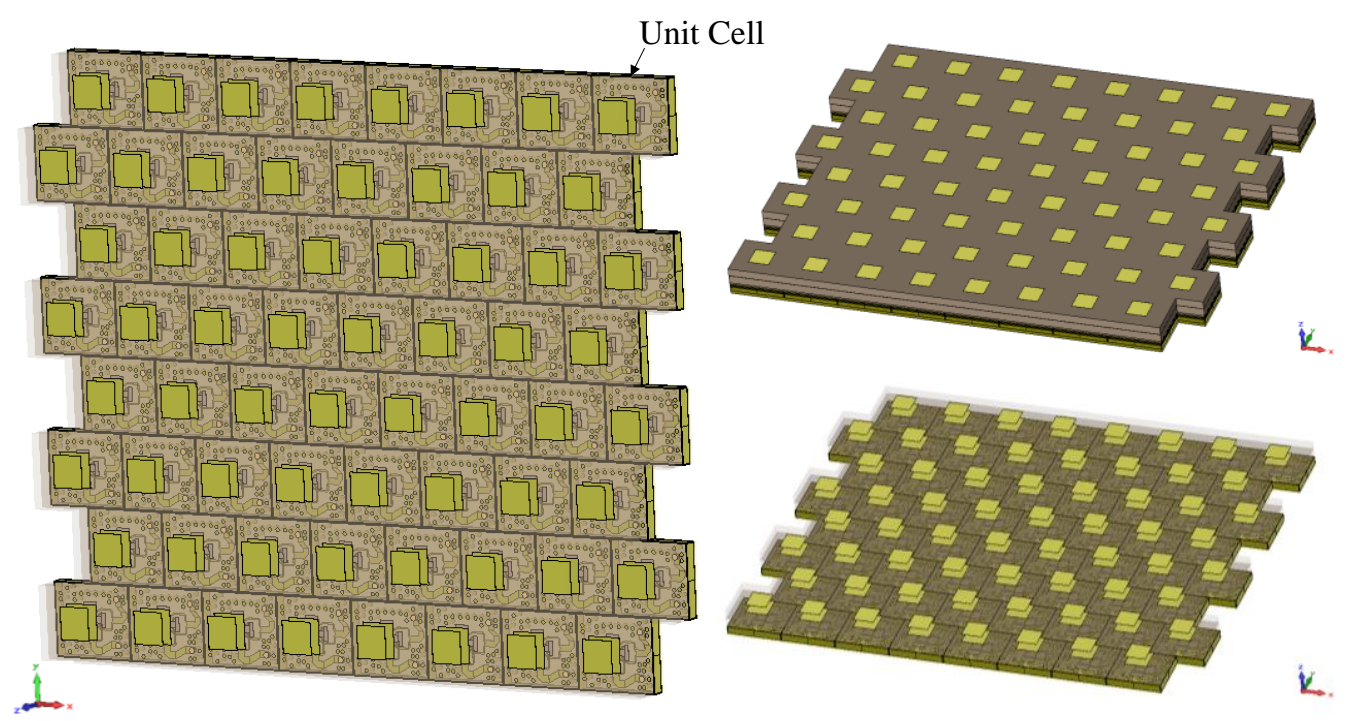

Figure 4.4.13 A 64-element array of unit cell antennas configured in an equilateral triangular lattice was constructed to demonstrate successful integration and operation of the unit cell antenna design in an array environment. 

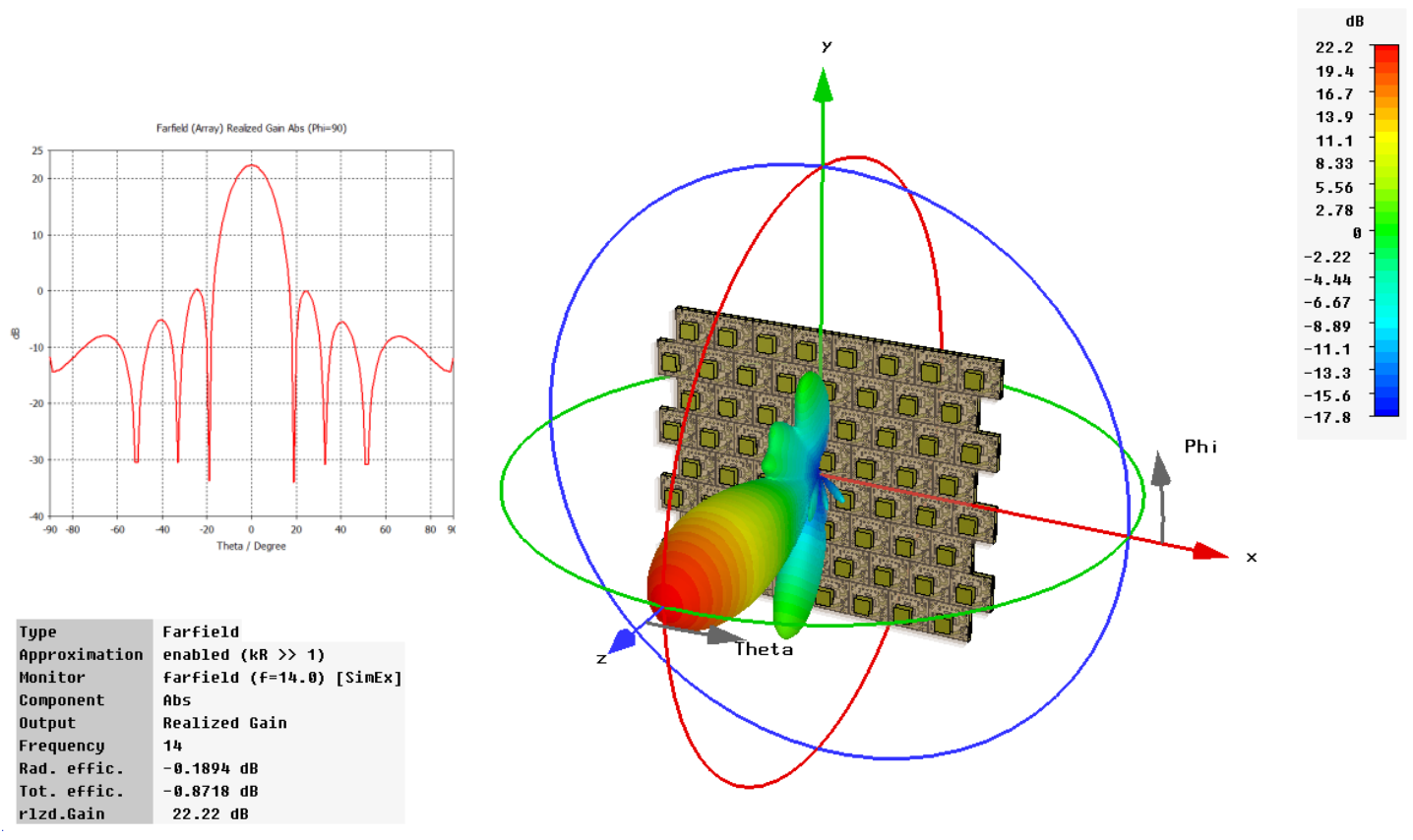

Figure 4.4.14 The computed 3D radiation pattern for a 64-element array using a $22 \mathrm{~dB}$ Taylor amplitude taper exhibits a realized gain of $22.2 \mathrm{dBi}$ at $14 \mathrm{GHz}$. This compares favorably to the expected realized gain which can be approximated as the element realized gain increased by the array gain and decreased by the amplitude taper loss; i.e., $6.3 \mathrm{dBi}+10 \log 64 \mathrm{~dB}-1.8 \mathrm{~dB}=$ $22.6 \mathrm{dBi}$. 


\section{Chapter 5}

\section{Conclusion}

\subsection{Project Summary}

This paper discusses the historical achievements leading to the parabolic reflector antenna, the importance of reflector antennas, and the advantages offered by phased array antennas. Key historical milestones include the investigation of conic sections and their interesting properties around $200 \mathrm{BC}$, the emergence of analytical geometry in the $17^{\text {th }}$ century, the invention of the reflecting telescope in 1668 , the formalization of electromagnetism in the late $19^{\text {th }}$ century, the first parabolic reflector antenna in 1888 , the invention of the phased array in 1905, and the first operational phased array system in 1937. The phased array antenna has revolutionized radar, radioastronomy, and satellite communication. Distinct advantages offered by the phased array include flexible beamforming, sidelobe control, and rapid beam scanning. The mathematics used to compute radiation properties of antennas and array systems are described.

Secondly, this work proposes a robust unit cell antenna design for an $\mathrm{X} / \mathrm{Ku}$-band satellite communications array application. The design employs an integrated stripline-fed slot-coupled 
stacked patch antenna and dual-stage branchline coupler utilizing multilayer PCB materials and fabrication processes. The integrated antenna and coupler fit within a 0.541 " by 0.469 " unit cell area and are less than 0.3 " thick. The design operates from 10.7 to $14.5 \mathrm{GHz}$ and enables a fullduplex array aperture which shares the receive band from 10.7 to $12.7 \mathrm{GHz}$ and transmit band from 13.7 to $14.5 \mathrm{GHz}$. A shared aperture topology increases design complexity, but reduces overall array cost and profile. The design achieves dual-circular polarization by employing a 90degree dual-stage branchline coupler at the output ports of the dual-linearly polarized antenna. A single-stage design cannot achieve the required bandwidth and RMS amplitude and phase errors, while a triple-stage design increases insertion loss and does not fit in a closely-space array grid. The dual-stage design achieves RMS amplitude and phase error of $0.16 \mathrm{~dB}$ and 0.63 degrees with port reflection and coupling coefficients less than $-15 \mathrm{~dB}$. Accommodating both circular polarizations allows the array system to implement electronically-rotatable linear polarization so that it can adapt to motion and continually realign its polarization. The antenna element achieves realized co-pol gain between 4.8 to $7.0 \mathrm{dBic}$ and maximum cross-pol level of $-17 \mathrm{~dB}$. Cleverly shaping the orthogonal feeds to minimize overlap and maximize distance between the tips of open circuit stripline stubs improves port isolation by $5-6 \mathrm{~dB}$ and cross-pol level by $2-3 \mathrm{~dB}$. A pair of 5-via transitions connects the hybrid to the antenna. A process known as backdrilling and backfilling removes unwanted via stubs and improves transition performance. Element pattern uniformity directly affects array-level performance. The proposed element pattern is symmetric and uniform for cardinal and diagonal planes across the operating bandwidth. An aperturecoupled feed helps maintain this uniformity by minimizing the unwanted interaction between both feeds and the antenna's radiation pattern. In contrast, the capacitively-coupled probe feeds employed in a prior design yields poor uniformity. In summary, the proposed design represents a substantial improvement in bandwidth, gain, and pattern uniformity relative to the prior design and meets the requirements of the satellite communications array application. 
A major challenge in PCB-based high frequency antenna design is minimizing performance degradation caused by materials and manufacturing process variance. A Monte Carlo investigation based on 100 simulation runs proves this design is much less sensitive to these tolerances than a prior design. The proposed design achieves less than $0.6 \mathrm{~dB}$ of co-pol gain variation and less than $4.0 \mathrm{~dB}$ of cross-pol gain variation. Performance most strongly varies with manufacturing variation, particularly misalignment between layers caused by registration inaccuracy when individual layers are laminated in the complete stackup.

Lastly, the antenna design exhibits stable performance in a closely-spaced array. Configured in a 0.541 " by $0.469 "$ equilateral triangular lattice, the array achieves a grating lobe free conical scan range of 48 degrees. A 64-element array simulated in Microwave Studio achieves a realized gain of $22.2 \mathrm{~dB}$ compared to a theoretical gain of $22.6 \mathrm{~dB}$.

\subsection{Future Extension}

This proposed design extends to applications across the SHF band from 3-30 GHz. The majority of modern array applications operate in this band. Materials and manufacturing process limitations generally limit performance at higher frequencies, although it is feasible to employ PCB-based antenna designs up to $60 \mathrm{GHz}$ for some applications. Satellite communication will continue to transition from $\mathrm{X} / \mathrm{Ku}$-band to Ka-band, so an extension of this work could explore the extensibility of this design and instantiate a unit cell operating at Ka-band frequencies. Another research alternative is to implement the same antenna topology in a more precise manufacturing process, such as wafer level micro-fabrication using equipment and processes used to manufacture semiconductors. It is likely that current state-of-the-art in PCB-based microwave phased arrays, illustrated in Figure 5.2.1, will be superseded by phased arrays based on microfabricated structures as illustrated in Figure 5.2.2. Micro-fabricated structures are processed with micron-level tolerances and can realize interconnects, transitions, chip carriers, and passive 
devices with excellent and repeatable performance. Rectangular coaxial transmission lines with $0.08 \mathrm{~dB} / \mathrm{cm}$ at Ka-band and $0.4 \mathrm{~dB} / \mathrm{cm}$ at $\mathrm{W}$-band have been demonstrated. Other key components in phased arrays, such as antennas, filters, chip sockets, power dividers, baluns, transitions, impedance transformers, and integrated PCB-like subassemblies have also been investigated [85]. The author is actively involved in this exciting research area and believes it is exceptionally promising for future microwave array applications operating at frequencies up to $200 \mathrm{GHz}$.

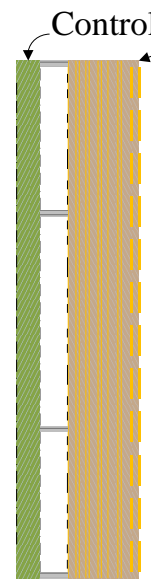

Side

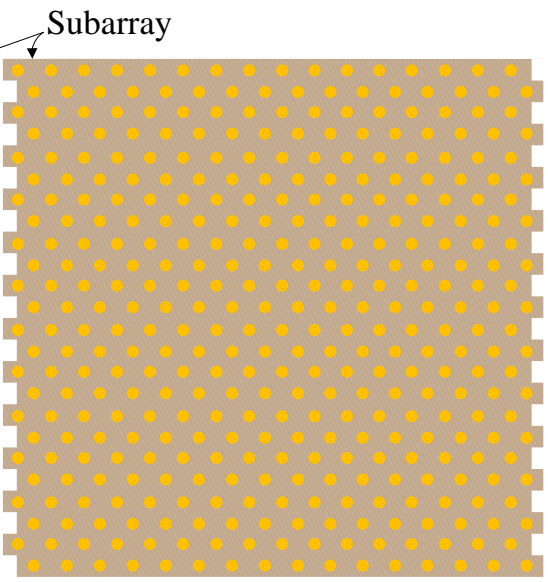

Front

(a)


Control and Subarray PCBs

(b)

Figure 5.2.1 The current state-of-the-art in tile-based array implementation. Pictured here is a 512-channel X-band ISR subarray with integrated SiGe beamforming RFICs and GaAs LNAs. The subarray and control PCBs are mated together with an aluminum frame. The overall thickness is less than 0.5 inches. 


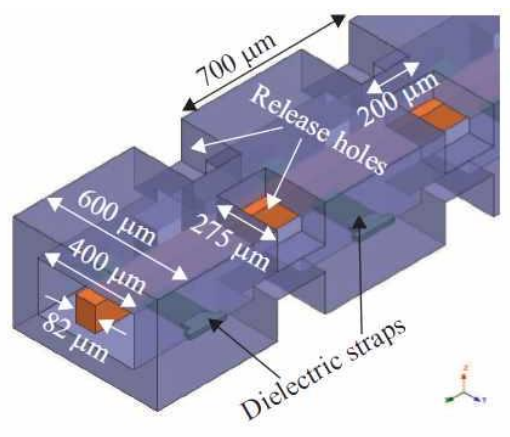

(a)

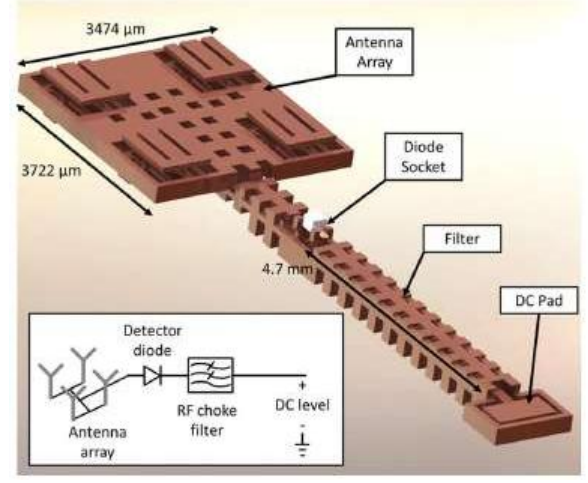

(c)

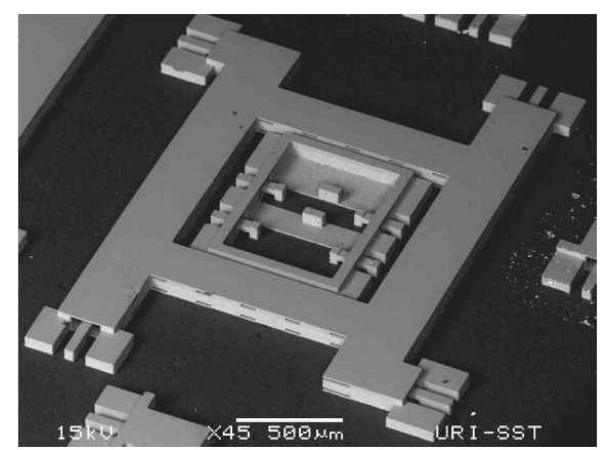

(b)

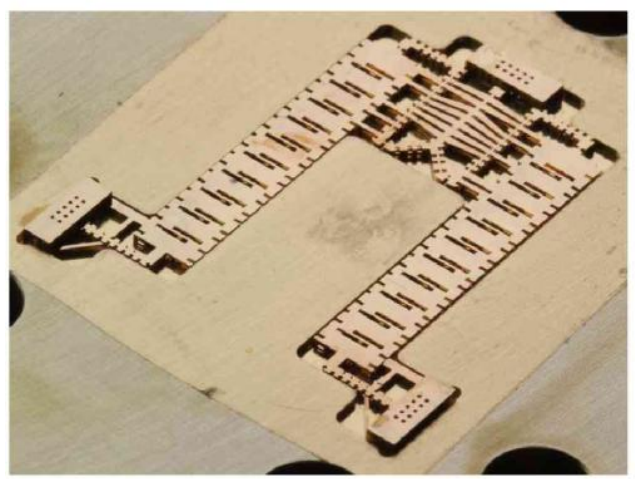

(d)

Figure 5.2.2 Micro-fabricated microwave structures are expected to revolutionize tile-type array implementations over the next two decades. Pictured here are (a) the physical geometry of an air-core rectangular coaxial transmission line from [85], (b) a micro-fabricated branchline coupler operating at $60 \mathrm{GHz}$ with groundsignal-ground probe interfaces from [86], (c) a 4-element array operating at 94 $\mathrm{GHz}$ using a cavity-backed patch antenna with $4.1 \%$ bandwidth and $8.3 \mathrm{dBi}$ gain at $95 \%$ efficiency from [87], and (d) a slot array operating from 87 to $102 \mathrm{GHz}$ achieving $14 \mathrm{~dB}$ gain at $94 \mathrm{GHz}$ and 30 degree scan angle over a $15 \mathrm{GHz}$ bandwidth from [88]. (All images: (C) IEEE) 


\section{Chapter 6}

\section{References}

\subsection{References}

[1] S. J. Orfanidis, Electromagnetic Waves and Antennas, Piscataway: Rutgers University, 2010.

[2] "Archimedes," [Online]. Available: http://www.britannica.com/. [Accessed 19 August 2012].

[3] Wikimedia Foundation, "Muhammad_ibn_Musa_al-Khwarizmi," 1 December 2012. [Online]. Available: http://en.wikipedia.org/wiki/Muhammad_ibn_Musa_al-Khwarizmi.

[4] A. Baranne and F. Launay, "Cassegrain: a famous unkown of instrumental astronomy," Journal of Optics, vol. 28, no. 4, pp. 158-172, 1997.

[5] F. L. Pedrotti, L. S. Pedrotti and L. M. Pedrotti, Introduction to Optics, Upper Saddle River: Pearson Education, Inc., 2007.

[6] NASA, "The James Webb Space Telescope," NASA, September 2009. [Online]. Available: www.jwst.nasa.gov. [Accessed 21 August 2012].

[7] J. C. Maxwell, "On Physical Lines of Force," Philosophical Magazine and Journal of 
Science, 1861.

[8] J. C. Maxwell, "A dynamical theory of the electromagnetic field," Philosophical Transactions of the Royal Society in London, no. 155, pp. 459-512, 1865.

[9] R. Evans, "Maxwell's Color Photography," Scientific American, no. 205, pp. 117-128, 1961.

[10] H. D. Young and R. A. Freedman, Sears and Zemansky's University Physics, 12th Edition, Glenview: Pearson Education, Inc., 2008.

[11] S. Hawking, A Stubbornly Persistent Illusion, Philadelphia: Running Press Book Publishers, 2007.

[12] M. Loomis, "Improvement in Telegraphing". United States Patent 129,971, 30 July 1872.

[13] W. H. Ward, "Improvement in Collecting Electricity for Telegraphing". United States Patent 126,356, 30 April 1872.

[14] A. G. Bell, "Apparatus for Signaling and Communicating, Called "Photophone."". Boston, Mass. Patent 235,119, 7 December 1880.

[15] D. E. Hughes, "Prof. D. E. Hughes' Research in Wireless Technology," The Electrician, vol. $43,1899$.

[16] W. L. Stutzman and G. A. Thiele, Antenna Theory and Design, Hoboken: John Wiley \& Sons, Inc., 1998.

[17] H. Hertz and D. E. Jones, Electric Waves, 2nd Ed., New York: McGraw-Hill, 1893.

[18] Wikimedia Foundation, "Heinrich Hertz," 5 December 2012. [Online]. Available: http://en.wikipedia.org/wiki/Heinrich_Hertz.

[19] Nobelprize.org, "The Nobel Prize in Physics 1909," 7 May 2013. [Online]. Available: http://www.nobelprize.org/nobel_prizes/physics/laureates/1909/ .

[20] National Radio Astronomy Observatory, "National Radio Astronomy Observatory Pioneers," Associated Universities, Inc, [Online]. Available: www.nrao.edu. [Accessed 02 Sept 2012].

[21] "Radio Telescopes Special Issue," IEEE Proc., vol. May, no. 88, pp. 629-829, 1994.

[22] S. Brunier, "Temples in the sky. Parts I and II.," Sky and Telescope, vol. Feb. \& June., no. 85, pp. 19-24, 26-32, 1993.

[23] A. Brandolini, S. Magri and T. M. Smeeding, "Asset-Based Measurement of Poverty," Institute for Research on Poverty, 2009. 
[24] Central Intelligence Agency, "The World Factbook," 24 August 2012. [Online]. Available: www.cia.gov. [Accessed 1 Sept 2012].

[25] Comision Federal de Telecomunicaciones, "Comunicado de Presna No. 21/2012," Distrito Federal, Mexico, 2012.

[26] J. D. Oetting and T. Jen, "The Mobile User Objective System," Johns Hopkins APL Technical Digest, vol. 30, no. 2, pp. 103-112, 2011.

[27] A. K. Bhattacharyya, Phased Array Antennas, Hoboken: Jon Wiley \& Sons, Inc., 2006.

[28] N. Fourikis, Advanced Array Systems, Applications, and RF Technologies, London: Academic Press, 2000.

[29] C. E. Shannon, "A mathematical theory of communication," Bell Sys. Tech J., vol. 27, pp. 379-423, 623-656, 1948.

[30] J. M. Wozencraft and I. M. Jacobs, Principles of Communication Engineering, Long Grove: John Wiley \& Sons, Inc. Reissued in 1990 by Waveland Press, Inc., 1965.

[31] C. A. Balanis, Antenna Theory: Analysis and Design, Hoboken: John Wiley \& Sons, Inc., 2005.

[32] N. Fourikis, Phased Array-Based Systems and Applications, New York: John Wiley \& Sons, Inc., 1997.

[33] P. e. a. Napier, "The very large baseline array," Proc. IEEE, vol. 2, no. 82, p. 658, 1994.

[34] H. T. Friis and C. B. Feldman, "A Multiple Unit Steerable Antenna for Short-Wave Reception," Proc. IRE, vol. 25, no. 7, pp. 841-917, 1937.

[35] H. T. Friis, "Radio System". United States of America Patent 2,041,600, 19 May 1936.

[36] E. Bruce and A. Beck, "Experiments with directivity steering for fading reduction," Proc. I.R.E., vol. 23, pp. 357-371, 1935.

[37] M. I. Skolnik, Introduction to Radar Systems, New York: The McGraw-Hill Companies, Inc., 2001.

[38] A. J. Fenn, "Evaluation of Adaptive Phased Array Antenna Far Field Nulling Performance Far Field Nulling Performance," Lincoln Laboratory, Lexington, 1989.

[39] S. Smith, "Optimum Phase-Only Adaptive Nulling," IEEE Trans. Sig. Proc., vol. 47, no. 7, 1999.

[40] D. Day, "Robust Phase-Only Nulling for Adaptive and Non-Adaptive Phased Arrays," in Proceedings 2007 Asilomar Conference on Signal, Systems and Computers, Monterey, 
2007.

[41] A. J. Fenn, D. M. Temme, W. P. Delaney and W. E. Courtney, "The Developments of Phased Array Radar Technology," Lincoln Laboratory Journal, vol. 12, no. 2, pp. 321-340, 2000 .

[42] L. O. Upton and L. A. Thurman, "Radars for the Detection and Tracking of Cruise Missiles," Lincoln Laboratory Journal, vol. 12, no. 2, 2000.

[43] B. D. Carlson, L. M. Goodman, J. Austin, M. W. Ganz and L. O. Upton, "An UltralowSidelobe Adaptive Array Antenna," Lincoln Lab Journal, vol. 3, no. 2, pp. 291-309, 1990.

[44] R. J. Mallioux, Phased Array Antenna Handbook, Norwood: Artech House Inc., 2005.

[45] W. J. Larson and J. R. Wertz, Space Mission Analysis and Design, 3rd Edition, New York: Microcosm Press \& Springer, 2006.

[46] M. Iskander, Electromagnetic Fields and Waves, Prospect Heights: Waveland Press, Inc., 2000 .

[47] W. K. H. Panofsky and M. Phillips, Classical Electricity and Magnetism, Mineola: Dover Publications, 2005.

[48] J. D. Kraus, Electromagnetics, New York: McGraw Hill Book Company, Inc., 1953.

[49] T. A. Milligan, Modern Antenna Design, Hoboken: Jon Wiley \& Sons, Inc., 2005.

[50] S. A. Schelkunoff, "A Mathematical Theory of Linear Arrays," Bell Syst. Tech. J., vol. 22, pp. 80-107, 1943.

[51] R. F. Harrington, "Antenna Excitation for Maximum Gain," IEEE Trans. Antennas Propagat., pp. 896-903, November 1965.

[52] W. W. Hansen and J. R. Woodyard, "A New Principle in Directional Antenna Design," Proc. IRE, vol. 26, pp. 333-345, 1938.

[53] A. Ishimaru, "Theory of Unequally Spaced Arrays," IRE Trans. Antennas Propagat., Vols. AP-10, pp. 691-702, 1962.

[54] H. Beljour, L. Coryell, T. Fung, J. Gallagher, R. Hoffmann, G. Michael and J. Shields, "Army SATCOM On The Move Technology Initiatives," IEEE Xplore, Ft. Monmouth, 2010.

[55] L. Gonzalez, G. Michael, J. Shields and C. Swenson, "Capacity and Regulatory Study On The Potential Use Of Satellite On the Move Terminals At X-Ban," in MILCOM 2008, 2008. 
[56] Computer Simulation Technology, "A Unit Cell Model of a Single Periodic Waveguide," 16 January 2006. [Online]. Available: www.cst.com. [Accessed 2012].

[57] C. J. Drane, "Useful approximations for the directivity and beamwidth of large scanning Dolph-Chebyshev Arrays," Proceedings of the IEEE, vol. 56, no. 11, pp. 1779-1787, 1968.

[58] R. J. Stegen, "The gain-beamwidth product of an antenna," IEEE Trans. Antennas and Propag., Vols. AP-12, pp. 505-506, 1964.

[59] D. Pozar, Microwave Engineering, Hoboken: John Wiley \& Sons, Inc., 2005.

[60] S. Slobin, C. Ho and K. Gritton, "Atmospheric Noise Temperature Induced by Clouds and Other Weather Phenomena at SHF Band (1-45 GHz)," JPL Publication D-32584, Edwards Air Force Base, 2005.

[61] J. D. Kraus, Radio Astronomy, Cygnus-Quasar Books, 1986.

[62] K.-J. Koh and G. M. Rebeiz, "An X- and Ku-Band 8-Element Phased-Array Receiver in 0.18-um SiGe BiCMOS Technology," IEEE Journal of Solid State Circuits, vol. 43, no. 6, pp. 1360-1371, 2008.

[63] D. M. Pozar, "Microstrip antenna aperture-coupled to a microstripline," IEEE Trans. Antennas \& Propagation, vol. 21, no. January, pp. 49-50, 1985.

[64] J. L. Volakis, Antenna Engineering Handbook, Fourth Edition, New York: The McGrawHill Companies, 2007.

[65] D. M. Pozar and D. H. Schaubert, Microstrip Antennas - The Analysis and Design of Microstrip Antennas and Arrays, Hoboken: John Wiley \& Sons, Inc., 1995.

[66] R. Bancroft, Microstrip and Printed Antenna Design, SciTech, 2009.

[67] D. L. Sherman, G. D. Hopkins and K. P. Pullen, "Microstrip Antenna System and Method". United States of America Patent 6,466,171, 15 October 2002.

[68] A. M. Puzella and F. Beltran, "Slot Coupled, Polarized, Egg-Crate Radiator". United States of America Patent 6,624,787, 23 September 2003.

[69] T. D. Huynh, "Dual Polarized Aperture Coupled Microstrip Patch Antenna System". United States of America Patent 5,896,107, 20 April 1999.

[70] C.-H. Tsao, Y. Hwang, F. J. Kilburg and F. J. Dietrich, "Planar Dual Polarization Antenna". United States of America Patent 4,903,033, 20 February 1990.

[71] S. D. Targonski, R. B. Waterhouse and D. M. Pozar, "Design of wide-band aperturestacked patch microstrip antennas," IEEE Trans. Antennas \& Propagation, vol. 46, no. September, pp. 1245-1251, 1998. 
[72] A. K. Bhattacharyya, O. Fordham and Y. Liu, "Analysis of stripline-fed slot-coupled patch antennas with vias for parallel-plate mode suppression," IEEE Trans. Antennas \& Propagation, vol. 46, no. April, pp. 538-545, 1998.

[73] R. B. Waterhouse, "Design and performance of large phased arrays of aperture stacked patches," IEEE Trans. Antennas \& Propagation, vol. 49, no. February, pp. 292-297, 2001.

[74] IBM Corporation, "Semiconductor Solutions: IBM Cu-45HP," April 2013. [Online]. Available: http://www.ibm.com.

[75] IBM Corporation, "Semiconductor Solutions: IBM Cu-32," April 2013. [Online]. Available: http://www.ibm.com.

[76] IBM Corporation, "Semiconductor Solutions: IBM SiGE BiCMOS 8HP," November 2012. [Online]. Available: http://www.ibm.com.

[77] F. Croq, G. Kossiavas and A. Papiernik, "Stacked resonators for bandwidth enhancement: a comparison of two feeding techniques," IEE Proceedings, vol. 140, no. August, pp. 303308, 1993.

[78] F. Klenfenz and A. Dreher, "Aperture-coupled stacked microstrip antenna with dual polarization and low back-radiation for $\mathrm{x}$-band SAR applications," Radio and Wireless Conference, pp. 179-182, 2000.

[79] D. Tallini, "A new low-profile wide-scan phased array for UWB applications," European Conf. on Antennas and Propagation, 2007.

[80] C. W. Sayre, Complete Wireless Design, Second Edition, New York: The McGraw-Hill Companies, Inc., 2008.

[81] Rogers Corporation, "High Frequency Materials Product Selection Guide," November 2010. [Online]. Available: http://www.rogerscorp.com.

[82] D. G. Swanson Jr and W. J. Hoefer, Microwave Circuit Modeling Using Electromagnetic Field Simulation, Norwood: Artech House, Inc., 2003.

[83] S. Gao, L. Li, S. Leong and T. Yeo, "A Broad-band Dual-polarized Microstrip Patch Antenna With Aperture Coupling," IEEE Trans. Antennas and Propoagation, vol. 51, no. 4, p. 898, 2003.

[84] B. Lindmark, "Antenna Element". United States of America Patent 6,018,319, 25 January 2000 .

[85] N. e. a. Ehsan, "Micro-Coaxial Lines for Active Hybrid-Monolithic Circuits," in International Microwave Symposium, 2009.

[86] J. R. Reid, E. D. Marsh and R. T. Webster, "Micromachined Rectangular-Coaxial 
Transmission Lines," IEEE Trans. on Microwave Theory and Techniques, vol. 54, no. 8, pp. 3433-3442, 2006.

[87] J. M. Oliver, J.-M. Rollin, K. Vanhille and S. Raman, "A W-Band Micromachined 3-D Cavity-Backed Patch Antenna Array With Integrated Diode Detector," IEEE Trans. on Microwave Theory and Techniques, vol. 60, no. 2, pp. 284-292, 2012.

[88] L. Ranzani, E. D. Cullens, D. Kuester, K. J. Vanhille, E. Grossman and Z. Popovic, "WBand Micro-Fabricated Coaxially-Fed Frequency Scanned Slot Arrays," IEEE Trans. on Antennas and Propag., vol. 61, no. 4, pp. 2324-2328, 2013.

[89] J. M. Yang, "Wafer Level Integrated Antenna Front End Module For Low Cost Phased Array Implementation," IEEE Microwave Symposium, vol. 14, pp. 1879-1881, 2007.

[90] H. A. Wheeler, "The Radiation Resistance of an Antenna in an Infinite Array or Waveguide," Proc. IRE, vol. 36, pp. 478-488, 1948.

[91] H. A. Wheeler, "The Grating-Lobe Series for the Impedance Variation in a Planar PhasedArray Antenna," IEEE Trans. Antennas and Propagation, vol. 14, pp. 707-714, 1966.

[92] H. A. Wheeler, "Simple Relations Derived From a Phased-Array Antenna Made of an Infinite Current Sheet," IEEE Trans. Antennas and Propagation, vol. 13, pp. 506-514, 1965.

[93] H. A. Wheeler, "A Systematic Approach to the Design of a Radiator Element for a PhasedArray Antenna," IEEE Proc., vol. 56, no. 11, pp. 1940-1951, 1968.

[94] B. C. Wadell, Transmission Line Design Handbook, Norwood: Artech House, Inc., 1991.

[95] S. Vaccaro, D. Llorens del Rio, R. T. Sanchez and R. Baggen, "Low cost phased array for mobile Ku-band satellite terminal," in 2010 Proceedings of the Fourth European Conference on Antennas and Propagation (EuCAP), 2010.

[96] J. S. Stone, "Radio Receiving System". United States of America Patent 1,954,898, 17 April 1934.

[97] L. Stark, "Microwave Theory of Phased-Array Antennas-A Review," Proceedings of the IEEE, vol. 62, no. 12, pp. 1660-1701, 1974.

[98] H. P. Stahl, "Survey of interferometric techniques used to test JWST optical components," Proc. SPIE 7790, p. 779002, 2010.

[99] J. G. Proakis and D. G. Manolakis, Digital Signal Processing, Upper Saddle River: Pearson Education, Inc., 2007.

[100] U. Lindgren and F. Athley, "Microwave Sparse Array Antenna Arrangement". United States of America Patent US 2010/0066635 A1, 18 March 2010. 
[101] J. Li and P. Stoica, "The Phased Array Is the Maximum SNR Active Array," IEEE Signal Processing Magazine, pp. 143-144, March 2010.

[102] B. P. Lathi, Modern Digital and Analog Communication Systems, Third Edition, New York: Oxford University Press, Inc., 1998.

[103] L. K. Lam, A. T. Ngo, M. S. Mahmoud and M. L. Peloquin, "Sparse Trifilar Array Antenna". United States of America Patent 7,466,287, 16 December 2008.

[104] D. G. Korsch, "Anastigmatic Three-Mirror Telescope". Huntsville, Alabama Patent 4,101,195, 18 July 1978.

[105] E. F. Knott, J. F. Shaeffer and M. T. Tuley, Radar Cross Section, Raleigh: SciTech Publishing, Inc., 2004.

[106] C. A. Harper, Electronic Assembly Fabrication, New York: The McGraw-Hill Companies, Inc., 2002.

[107] R. C. Hansen, Phased Array Antennas, New York: John Wiley \& Sons, Inc., 1998.

[108] J. P. Gardner et. al., "The James Webb Space Telescope," Springer, 2006.

[109] S. Foo, "A dual-polarized CPW-fed aperture coupled stacked patch," in 13th IEEE Trans. Symposium Antenna Technology, 2009.

[110] S. Edelberg and A. A. Oliner, "Mutual Coupling Effects in Large Antenna Array," IRE Trans., vol. 8, pp. 286-297, 1960.

[111] K. Chang, Microwave Solid-State Circuits and Applications, New York: John Wiley \& Sons, Inc., 1994.

[112] National Radio Astronomy Observatory, "Iconic Telescope Renamed to Honor Founder of Radio Astronomy," Associated Universities, Inc., 10 January 2012. [Online]. Available: http://www.nrao.edu/pr/2012/jansky/.

[113] "Archimedes Death Ray: Idea Feasibility Testing," Massachusetts Institute of Technology, October 2005. [Online]. Available: http://web.mit.edu/2.009/www/experiments/deathray.

[114] Wikimedia Foundation, "Speculum Metal," 5 April 2013. [Online]. Available: http://en.wikipedia.org/wiki/Speculum_metal. 


\section{Chapter 7}

\section{Appendices}

\subsection{MATLAB Code}

The following code has been written to facilitate studies and generate figures in this work.

The code is organized by subject and categorized as scripts or functions. 


\subsubsection{Array Radiation Pattern Functions}

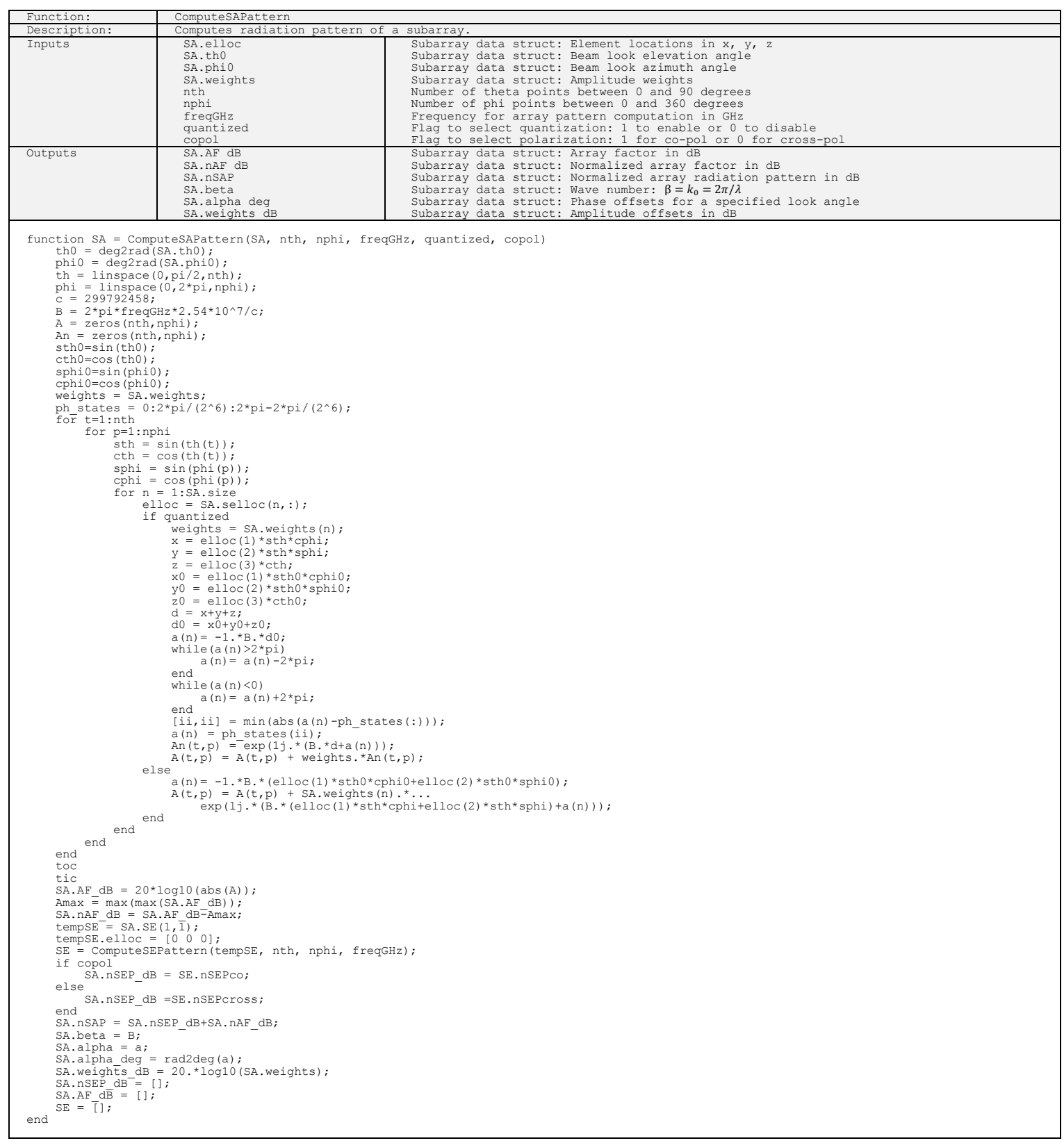

\subsubsection{Element Radiation Pattern Functions}

\begin{tabular}{|c|c|c|}
\hline Function: & $\begin{array}{l}\text { Format Element Pattern } \\
\text { Local helper functions: ScanPatternFile, Com }\end{array}$ & arePatternData, ProcessPatternFile, FormatPlots, Figurecut \\
\hline Description: & Translates the 3D radiation pattern from CST & Microwave Studio to MATLAB \\
\hline Inputs & $\begin{array}{l}\text { fname } \\
\text { Nphi } \\
\text { Nth } \\
\text { comp } \\
\text { show } \\
\text { varargin }\end{array}$ & $\begin{array}{l}\text { Subarray data struct: Element locations in } x, y, z \\
\text { Subarray data struct: Beam look elevation angle } \\
\text { Subarray data struct: Beam look azimuth angle } \\
\text { Flag to plot patterns for verification: "plotall" to enable } \\
\text { Flag to enable file comparison: "noshow" to bypass } \\
\text { Unused - utilized in later iteration of code }\end{array}$ \\
\hline Outputs & $\begin{array}{l}\text { Struct. Raw co dB } \\
\text { Struct. Raw cross dB } \\
\text { Struct. Raw nth } \\
\text { Struct. Raw nphi } \\
\text { Struct.Proc co dB } \\
\text { Struct.Proc cross dB } \\
\text { Struct.Proc nth } \\
\text { Struct.Proc nohi }\end{array}$ & $\begin{array}{l}\text { CST pattern file: co-pol element pattern in } \mathrm{dB} \\
\text { CST pattern file: cross-pol element pattern in } \mathrm{dB} \\
\text { CST pattern file: number of theta points } \\
\text { CST pattern file: number of phi points } \\
\text { Processed pattern file: co-pol element pattern in } \mathrm{dB} \\
\text { Processed pattern file: cross-pol element pattern in } \mathrm{dB} \\
\text { Processed pattern file: number of theta points } \\
\text { Processed pattern file: number of phi points }\end{array}$ \\
\hline \multicolumn{3}{|c|}{$\begin{array}{l}\text { function OutputStruct = FormatElementPattern (fname, Nphi, Nth, comp, show, varargin) } \\
\text { try } \\
\text { fp = fopen (fname); } \\
\text { ProcInput = ScanPatternFile (fp, fname); }\end{array}$} \\
\hline
\end{tabular}




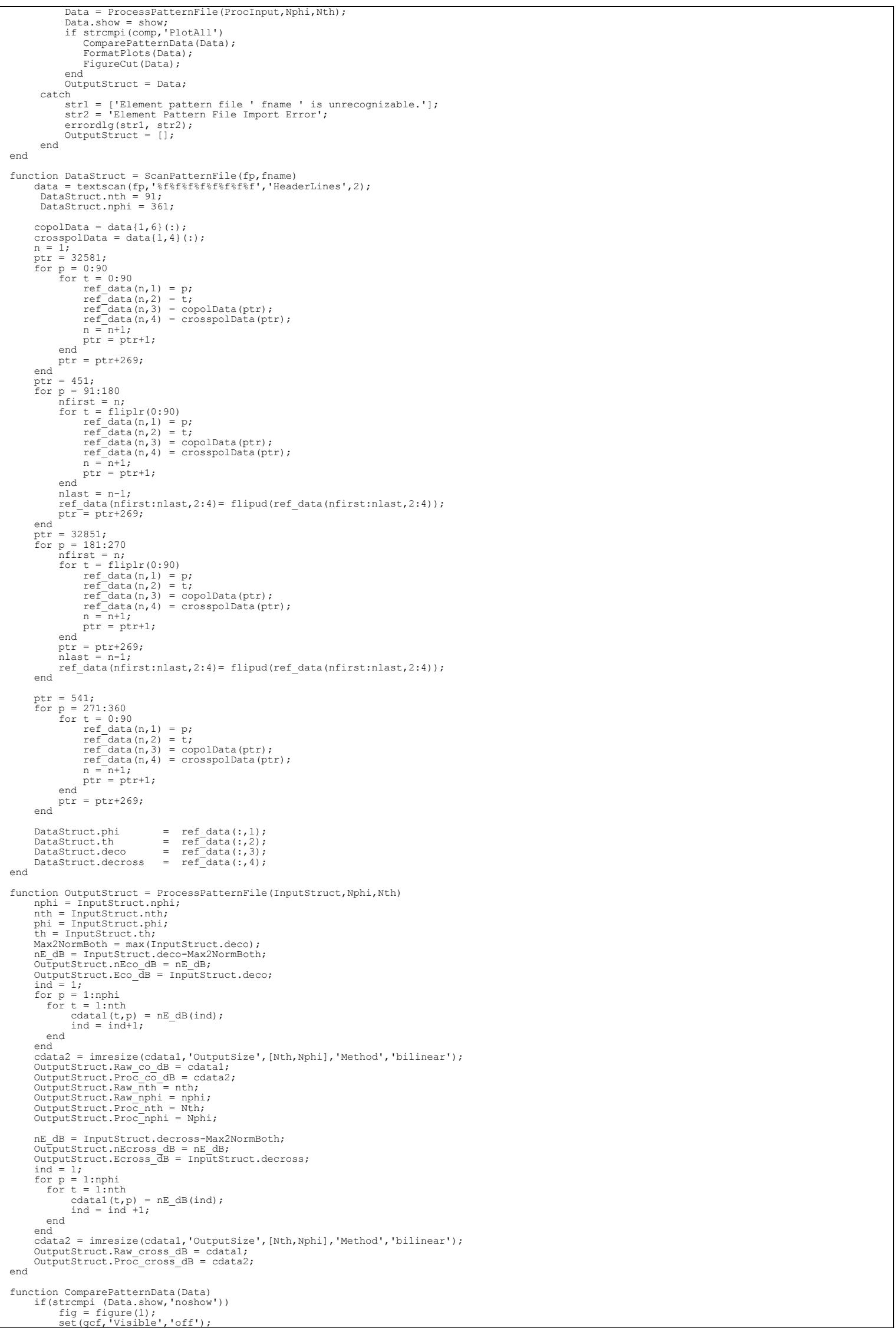




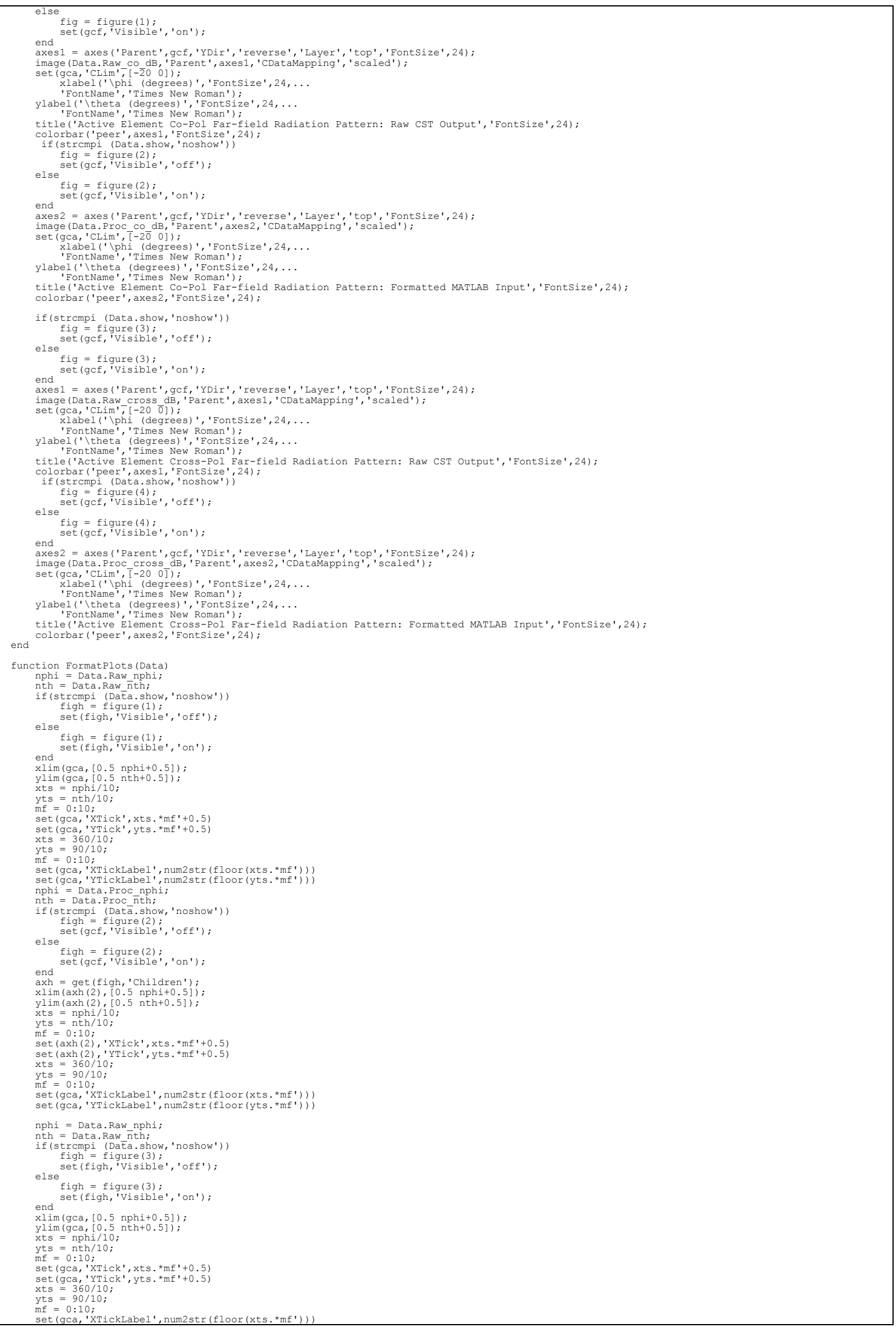




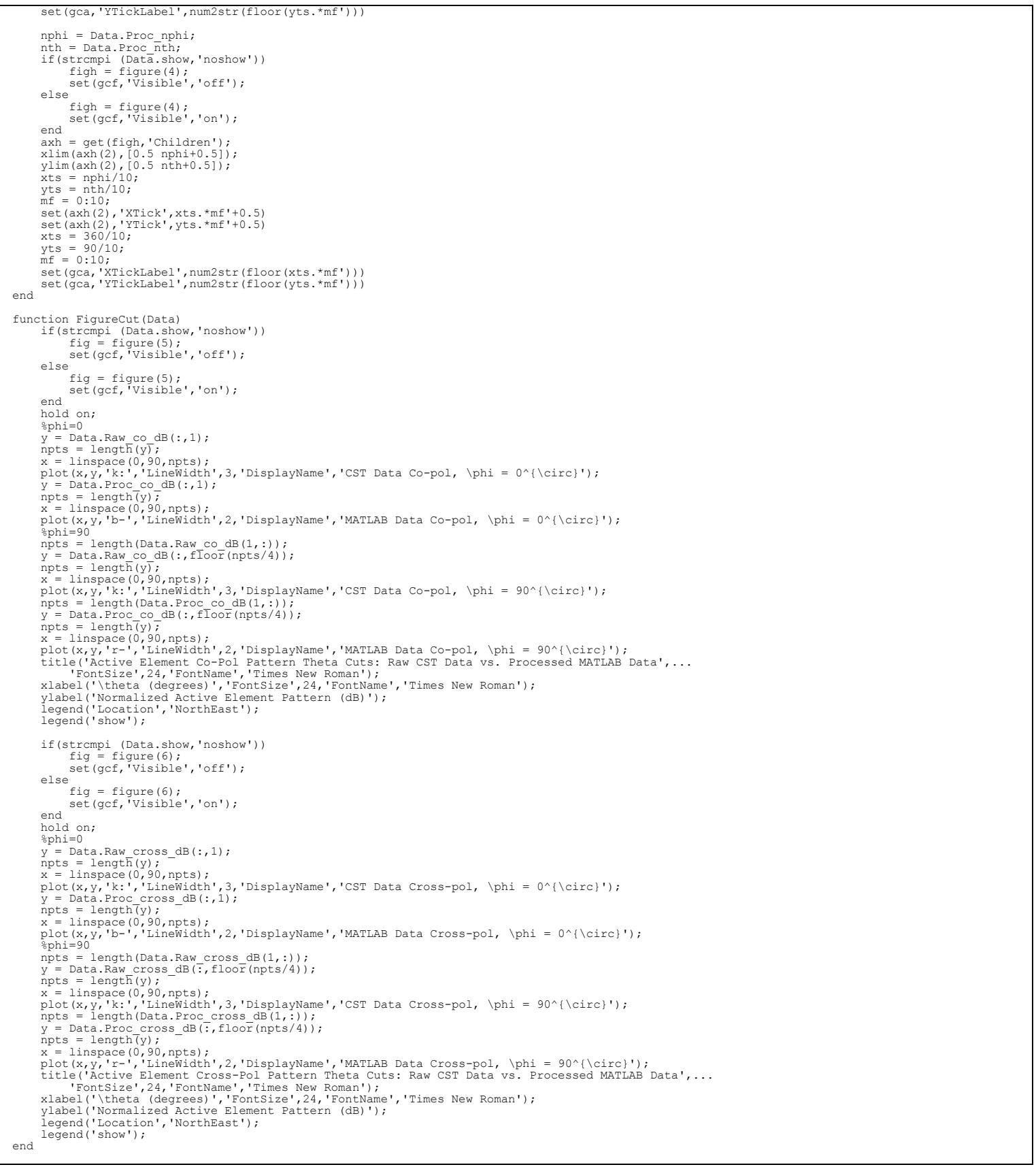

\subsubsection{Hertzian Dipole Visualization Functions}

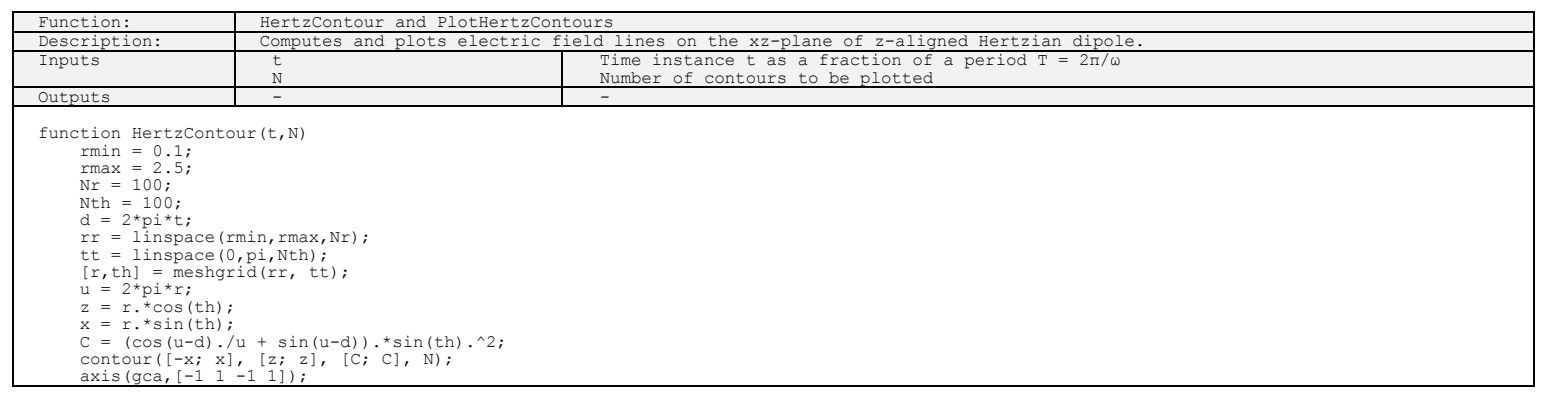




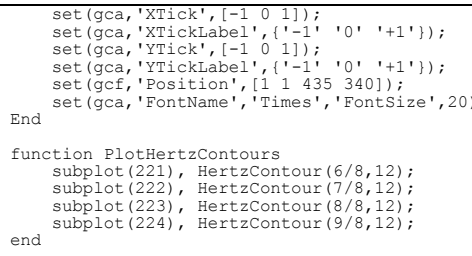

\subsubsection{S-parameter Processing \& Plotting Scripts}

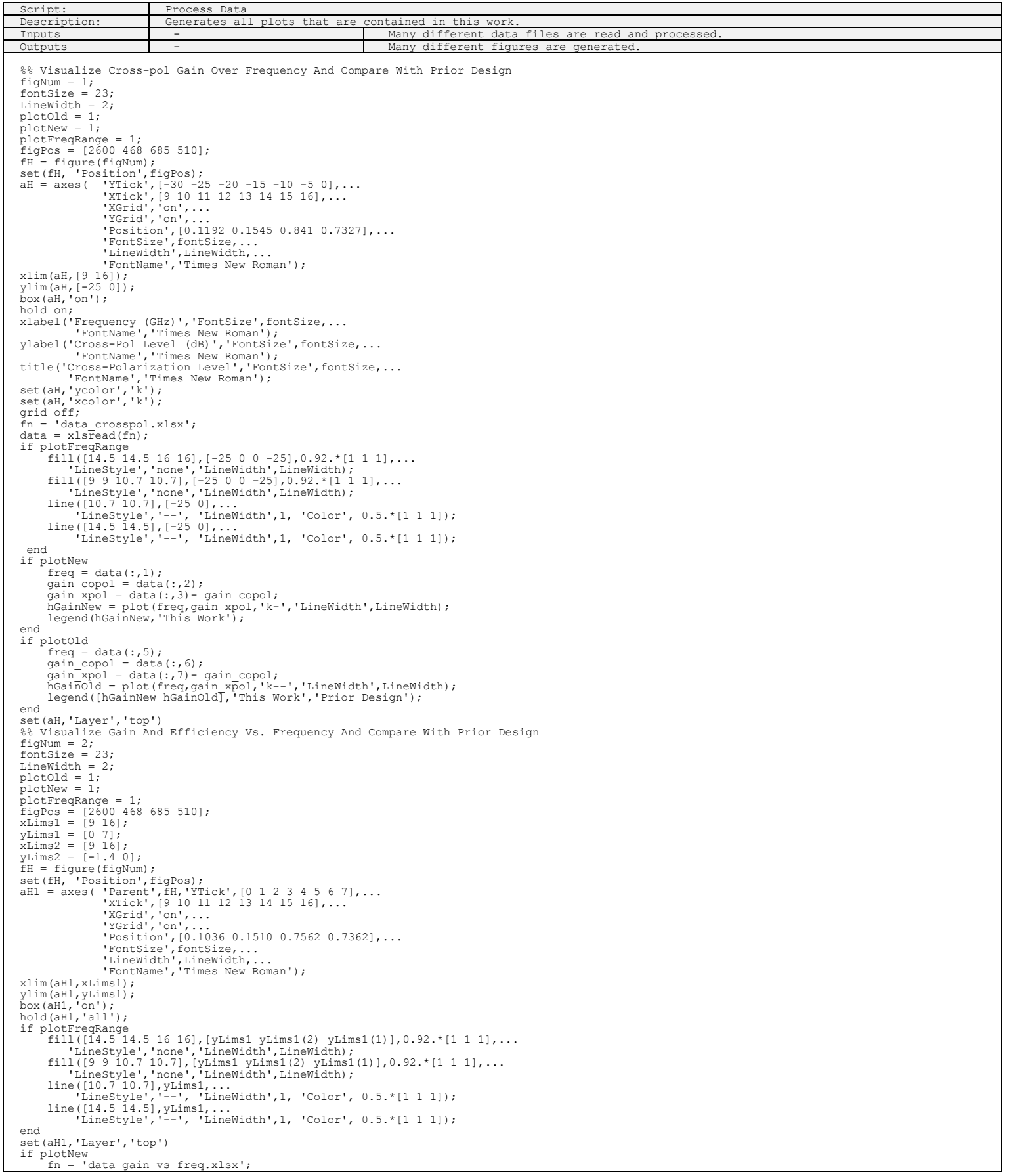




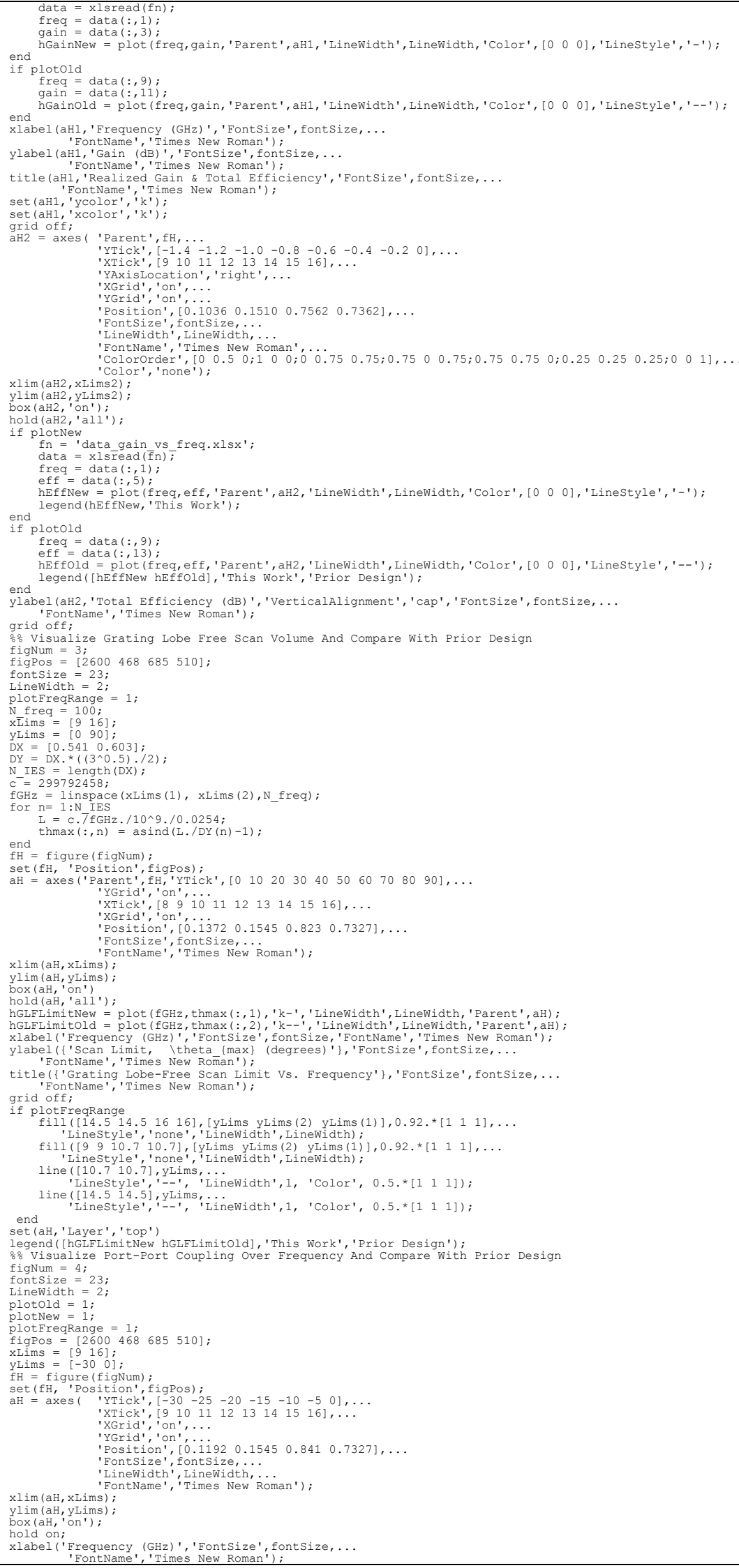









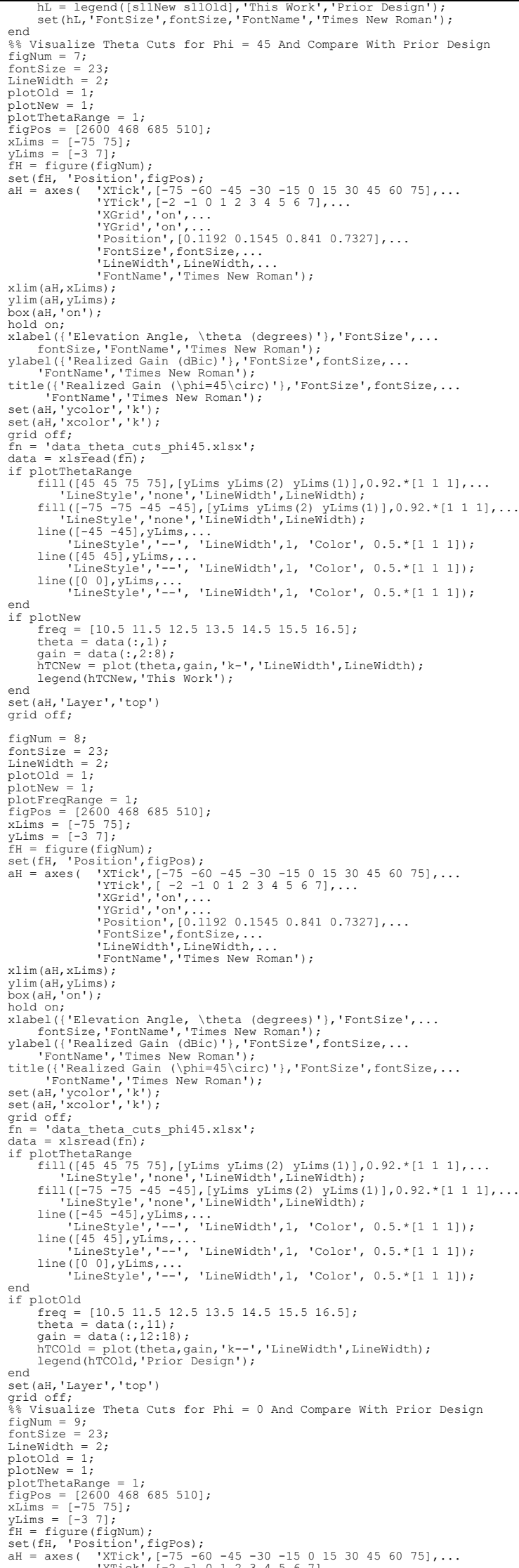




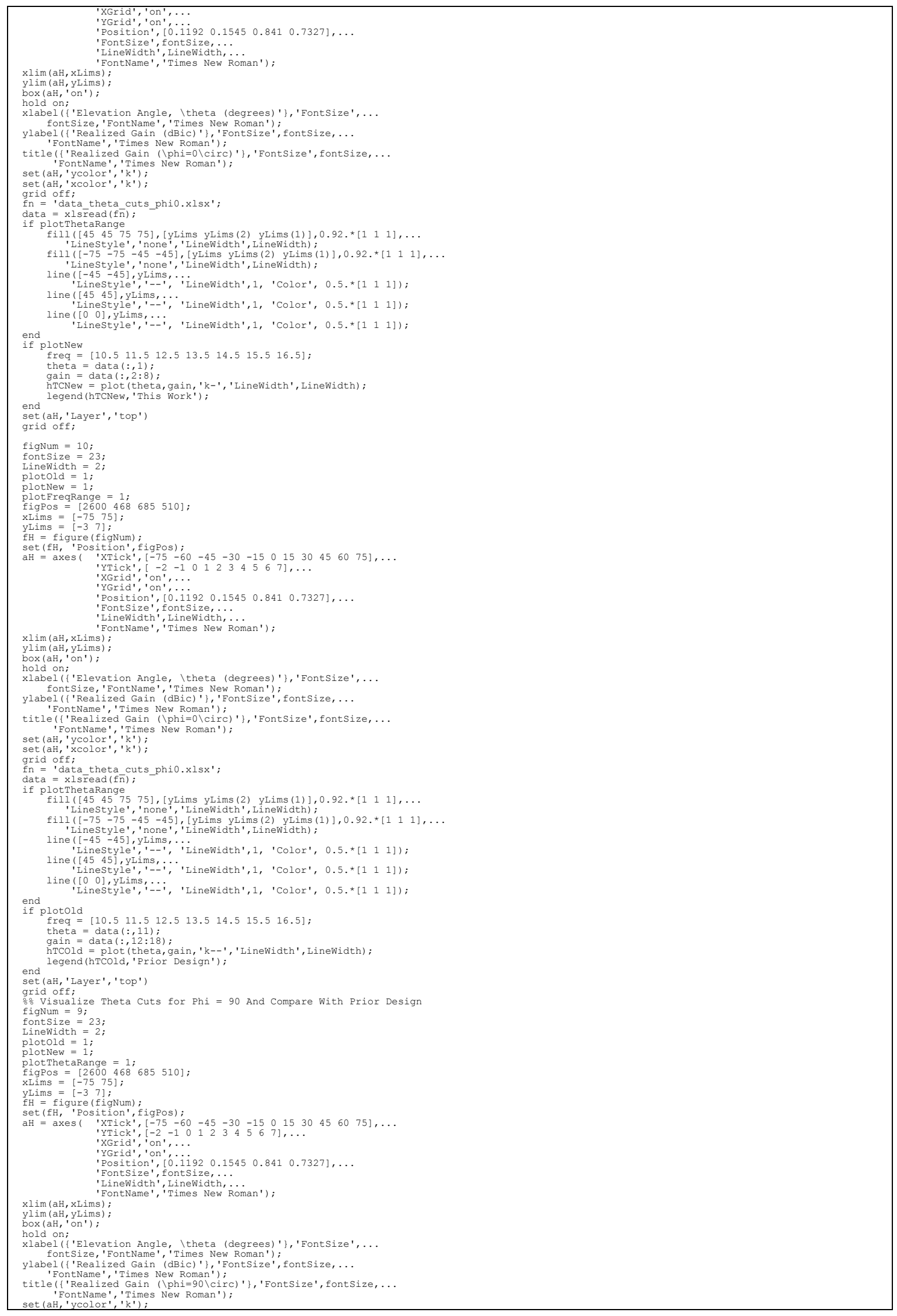




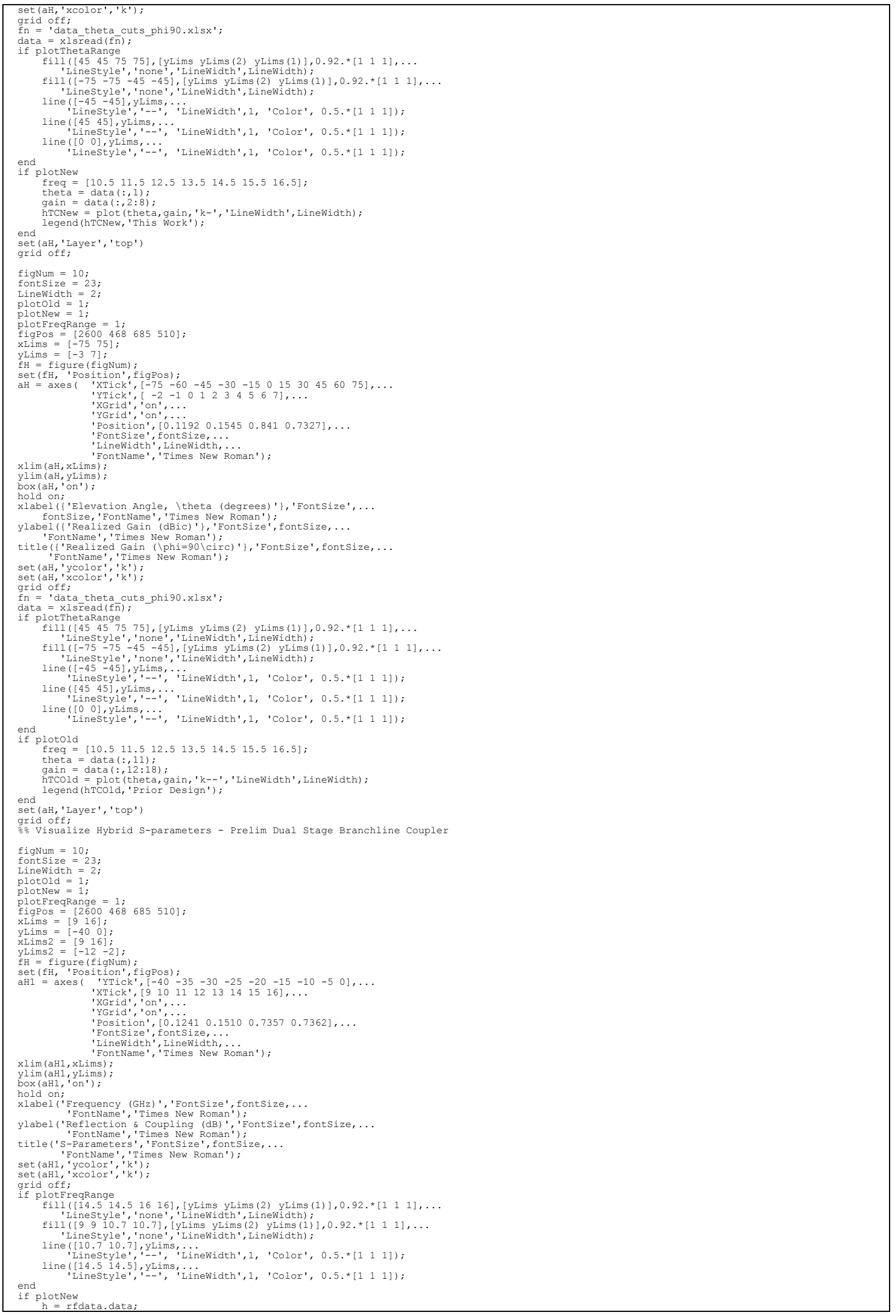




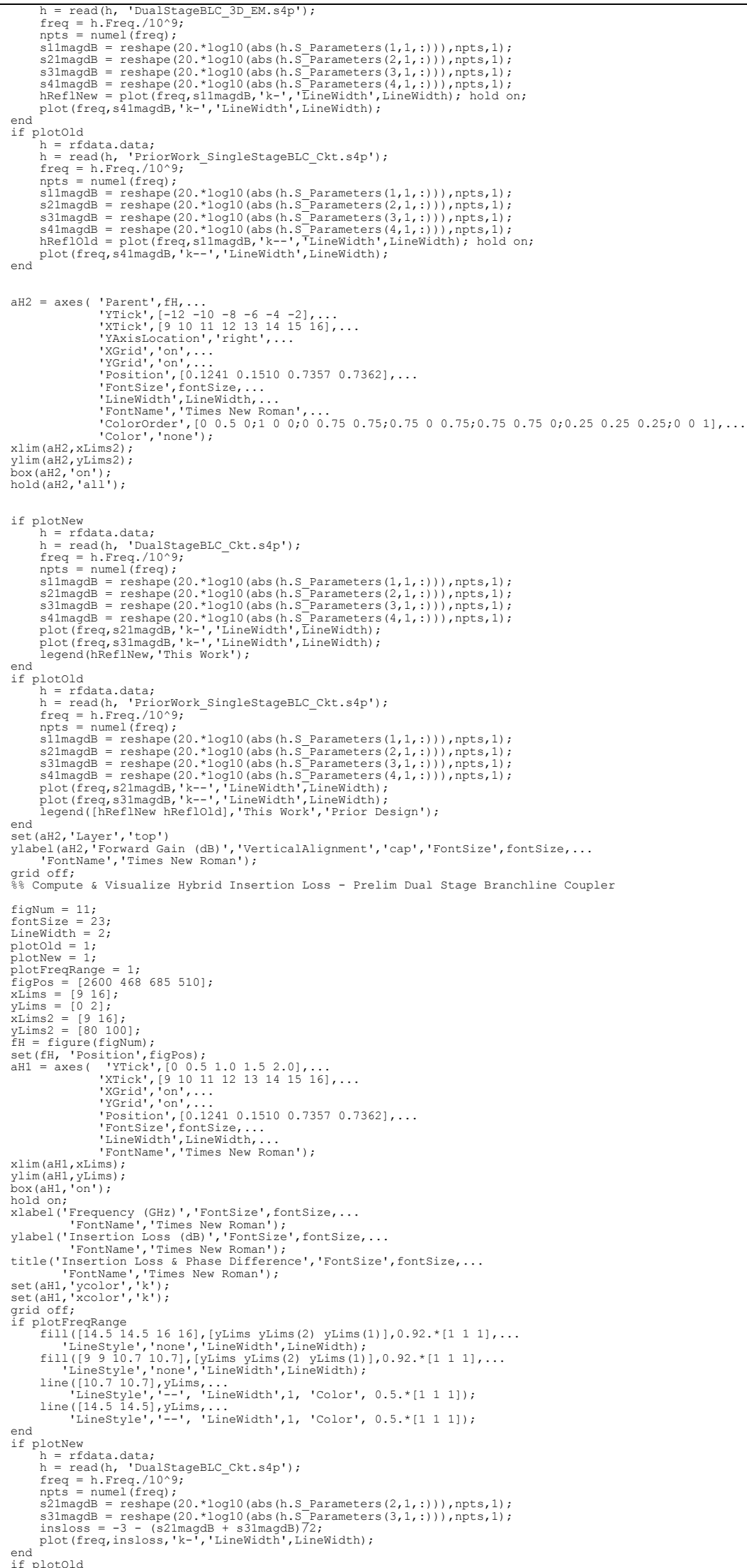









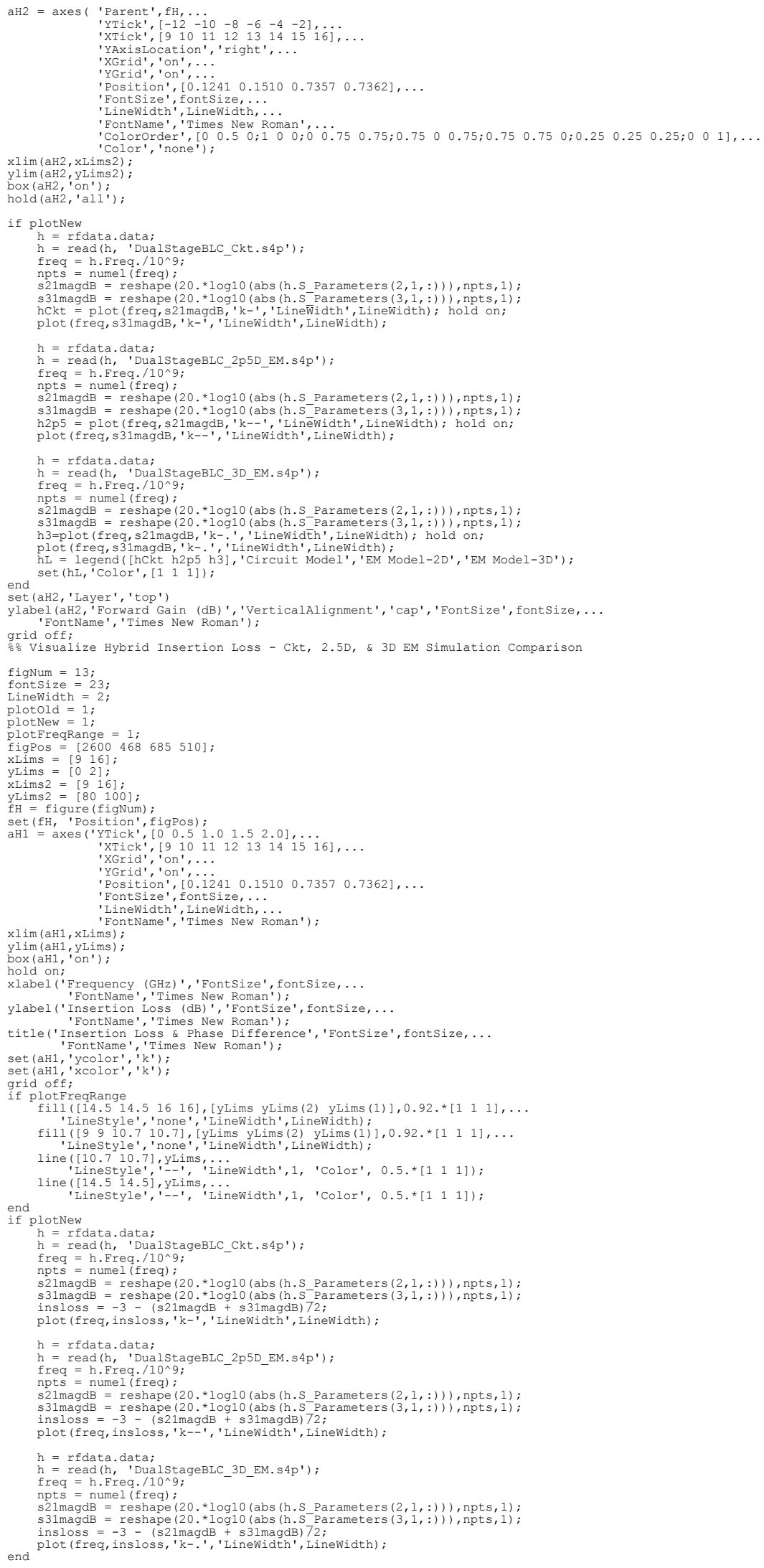




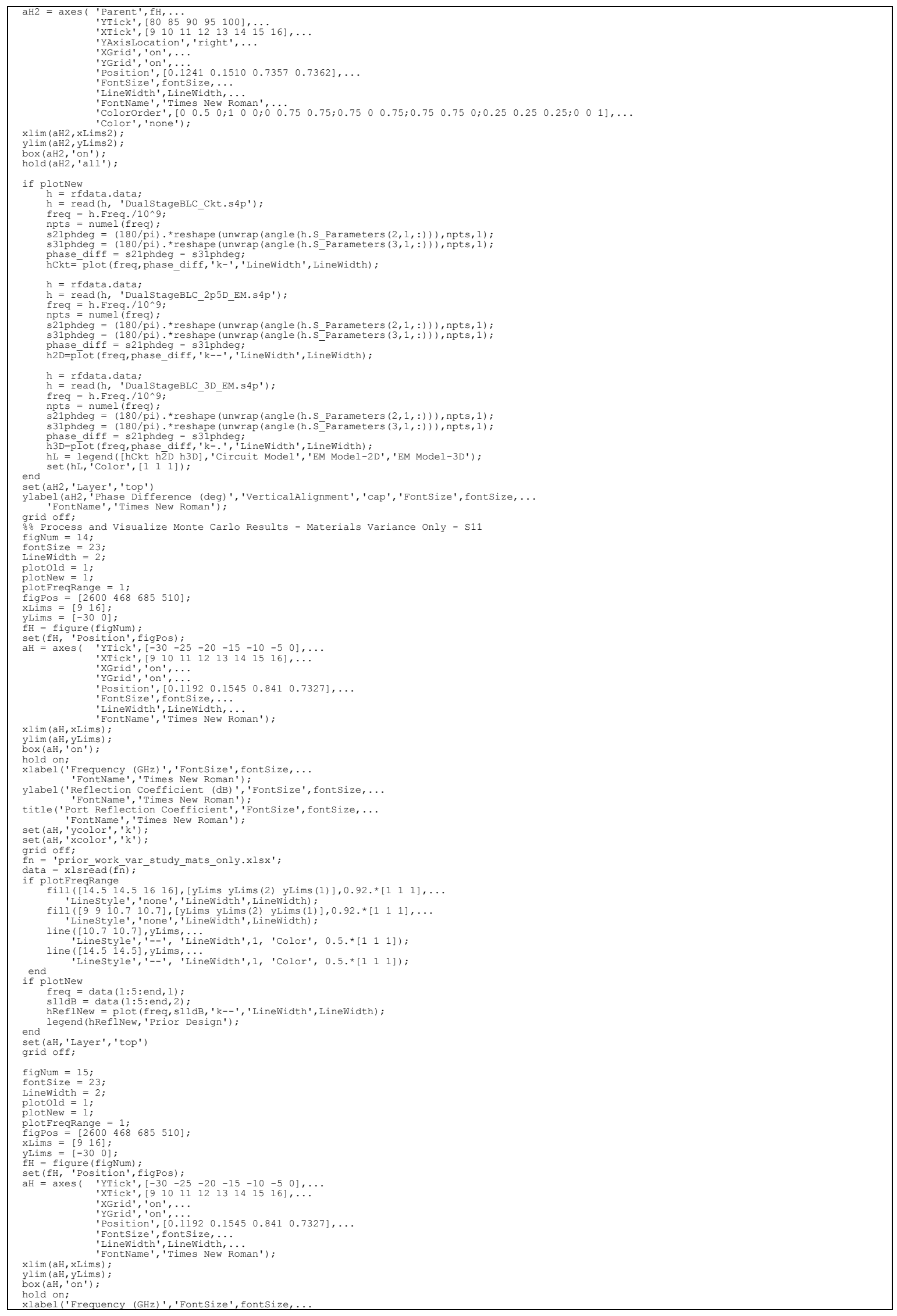




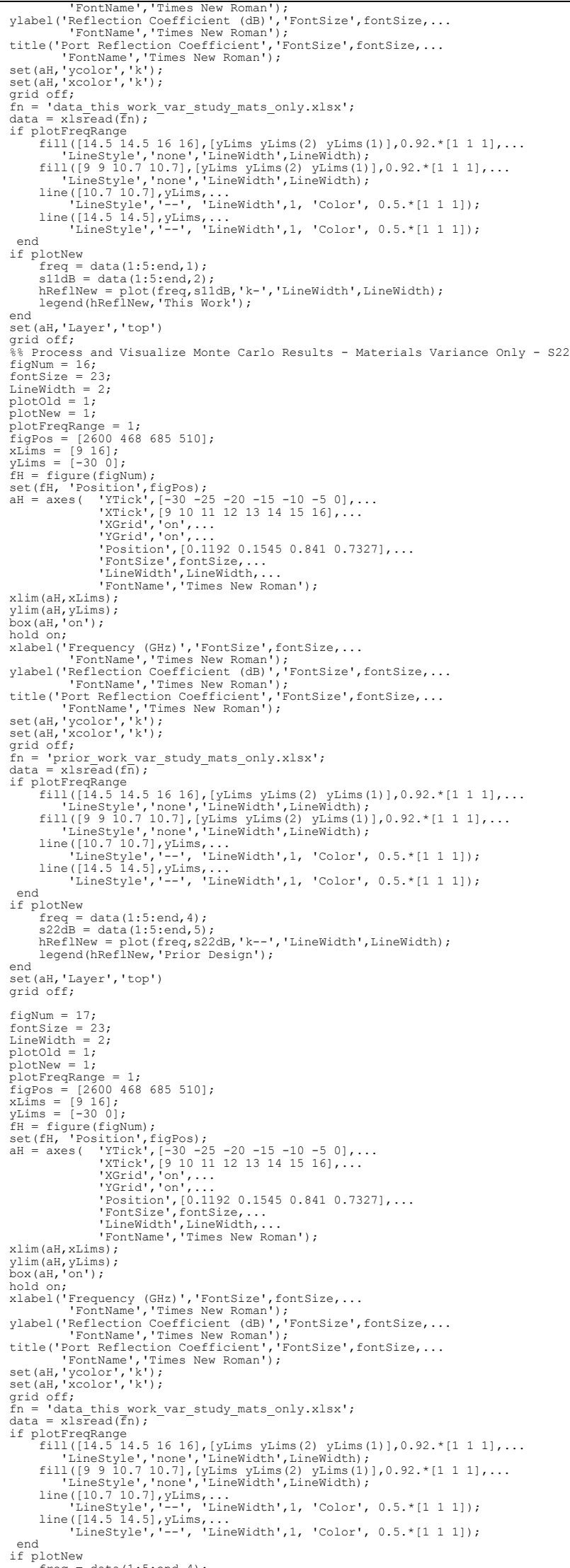




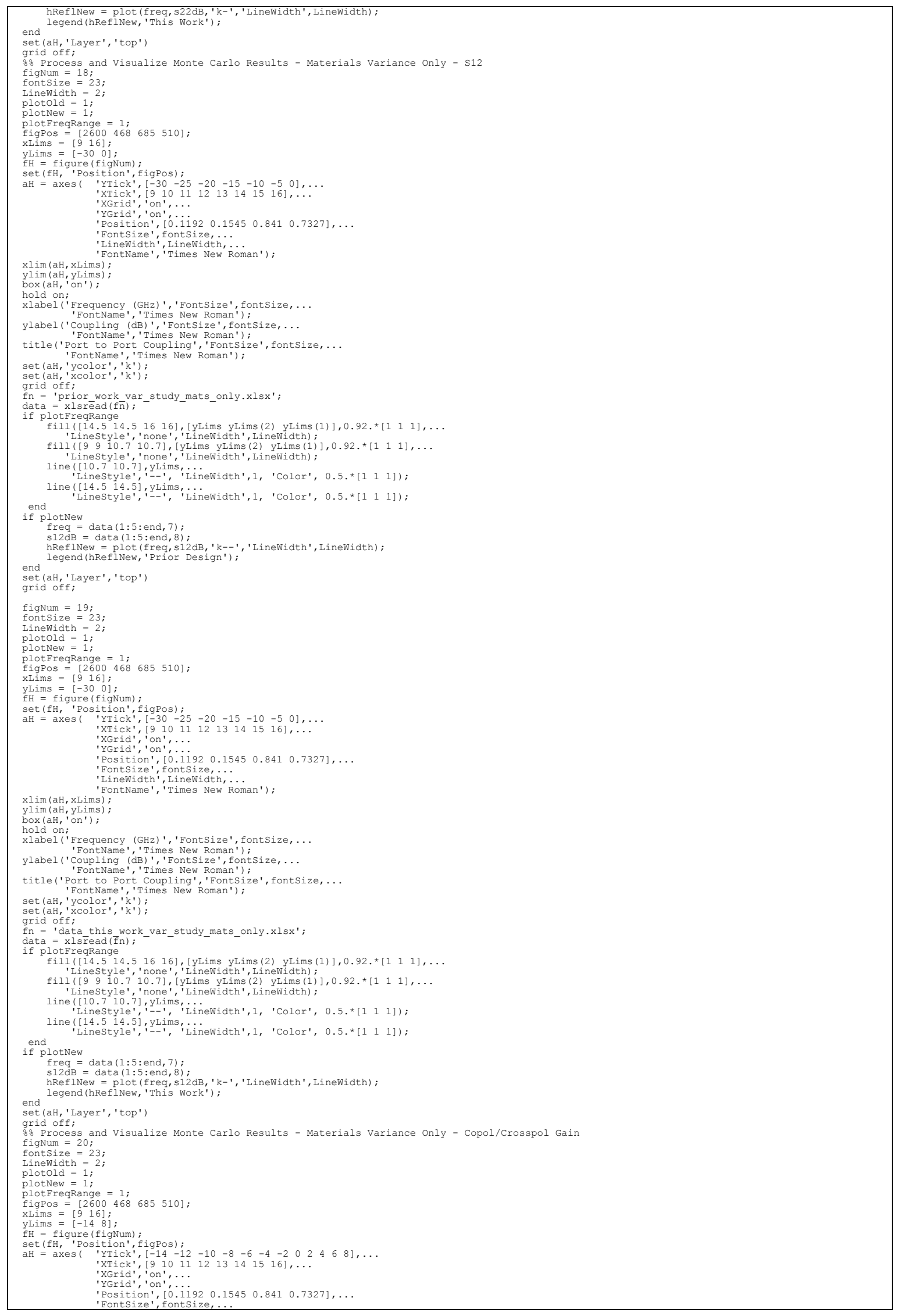




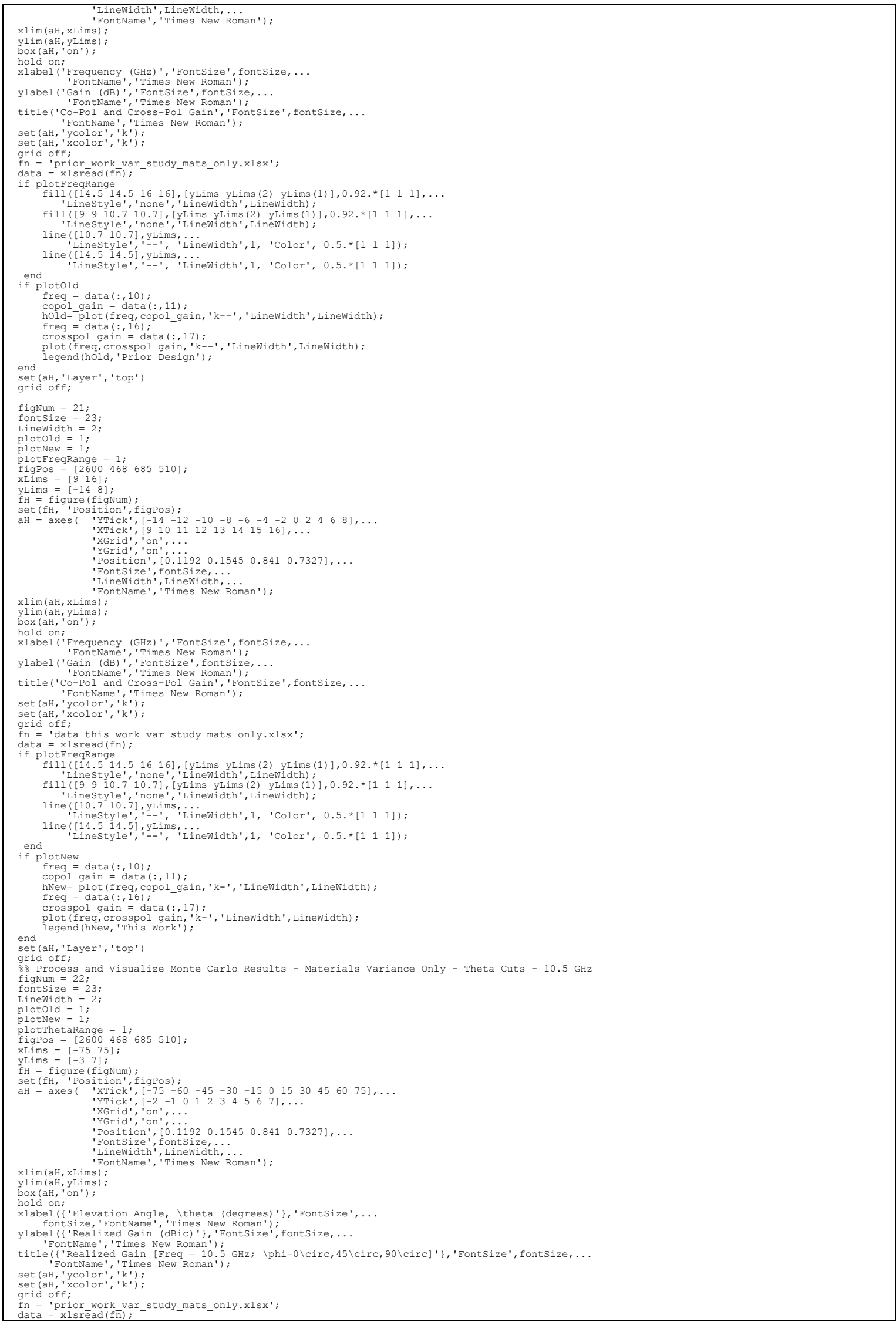




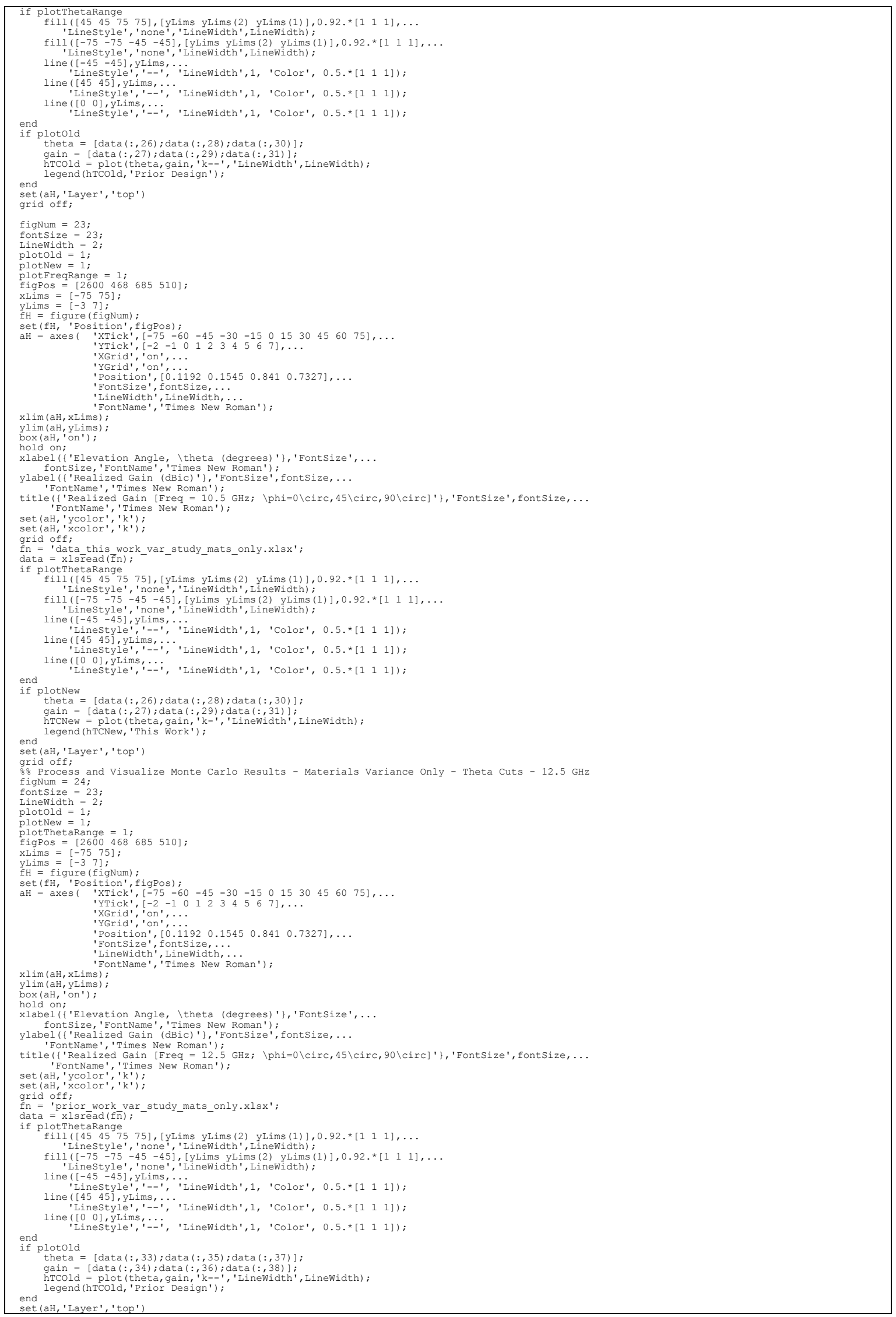




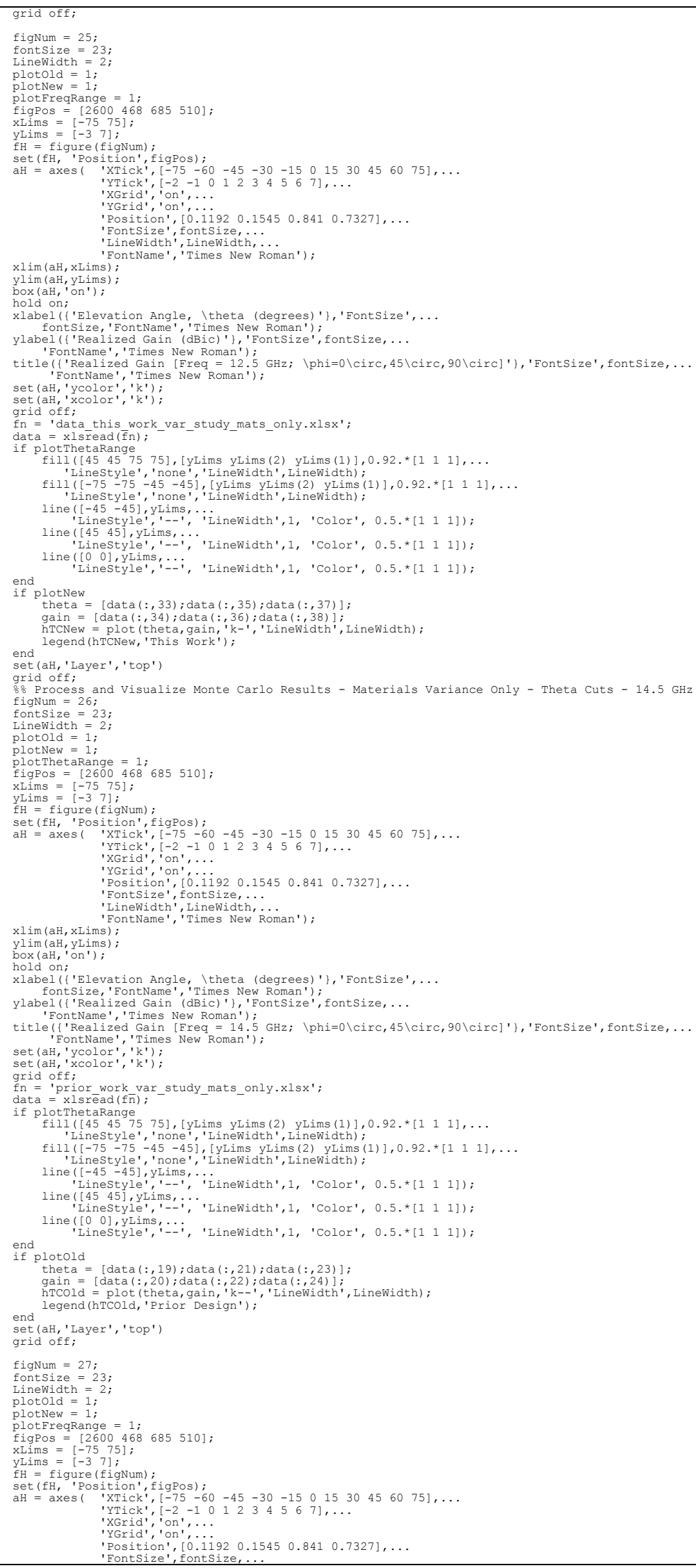




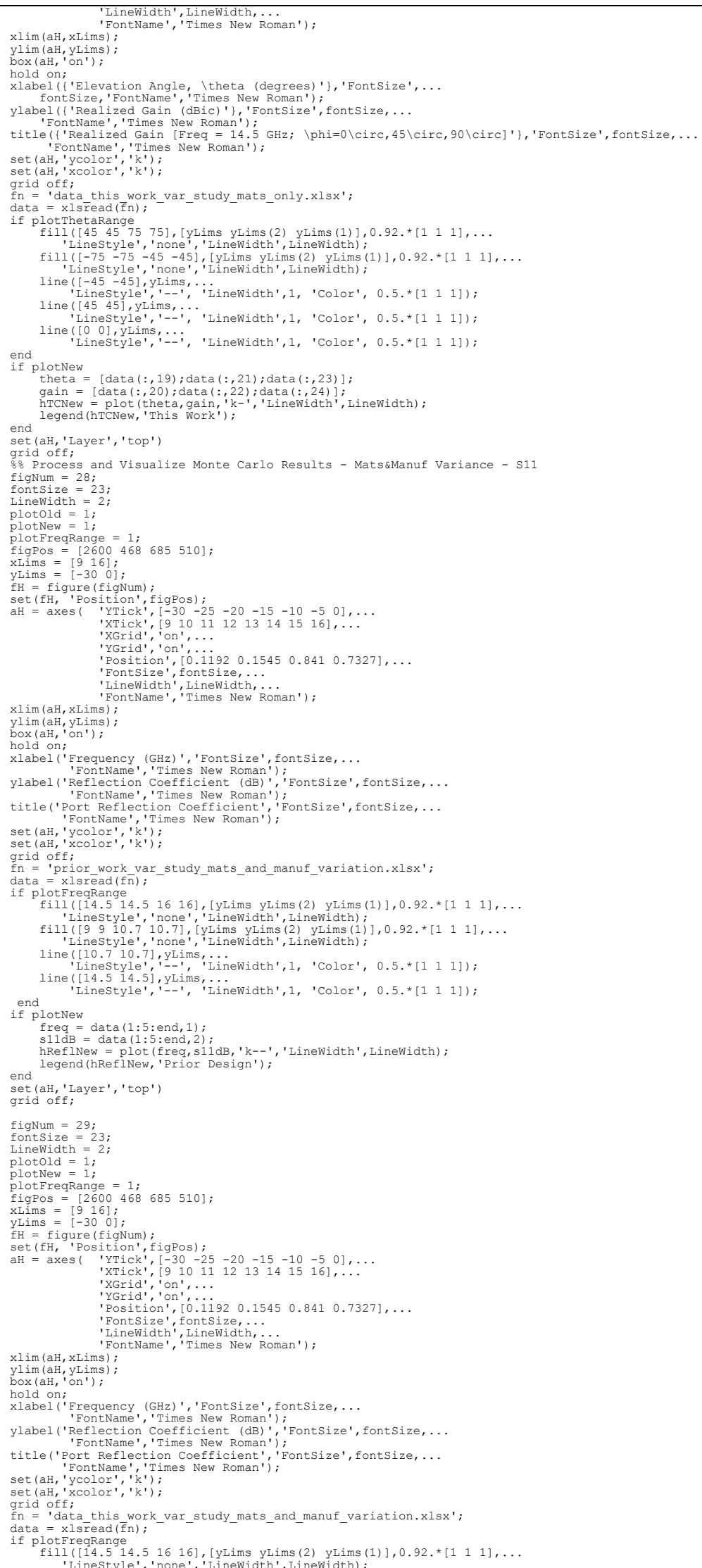




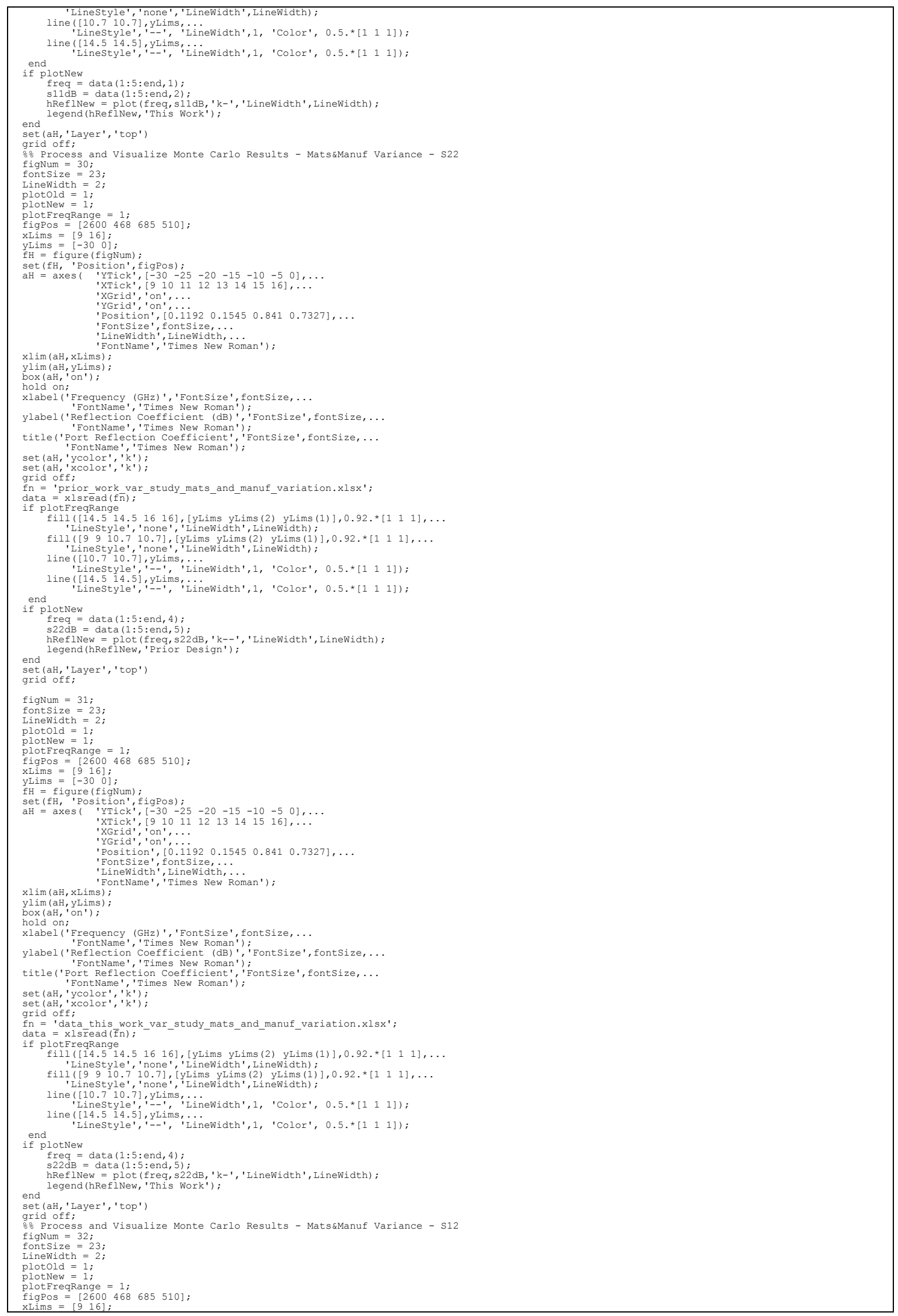




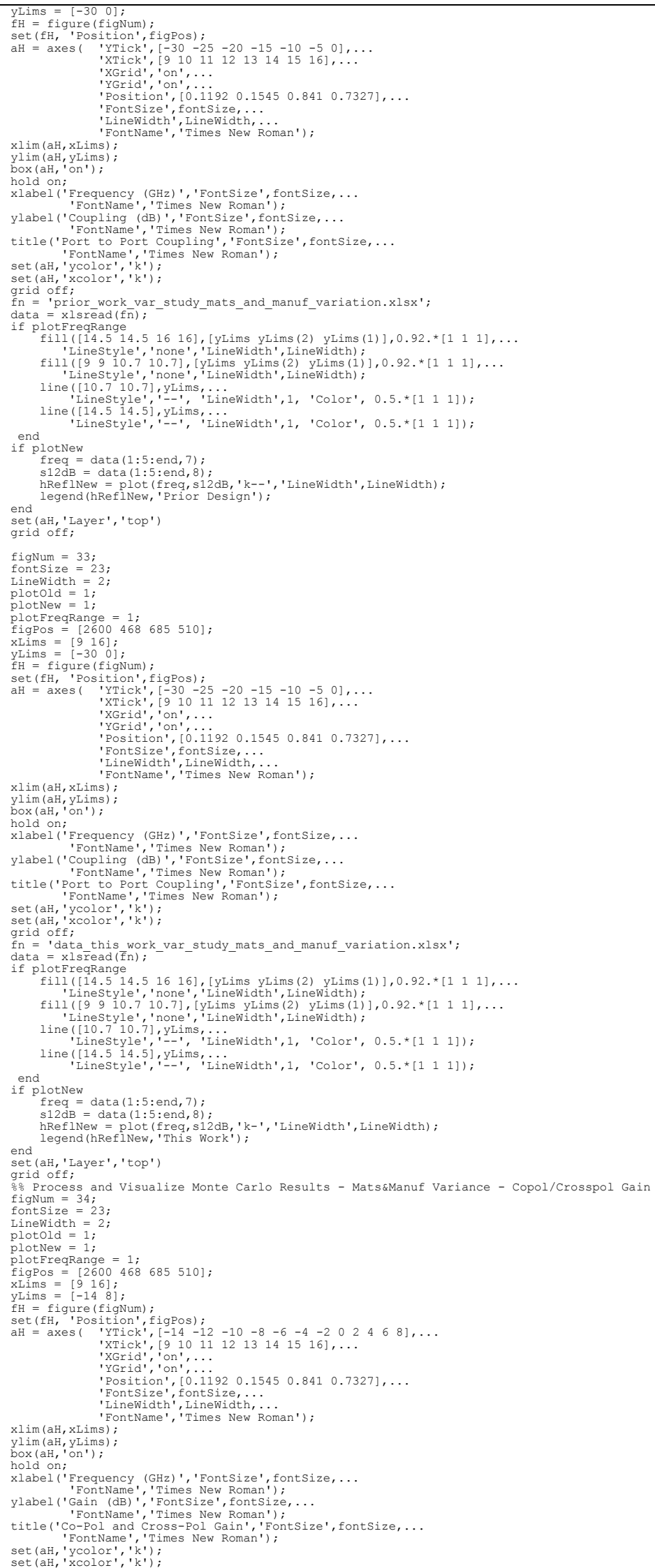




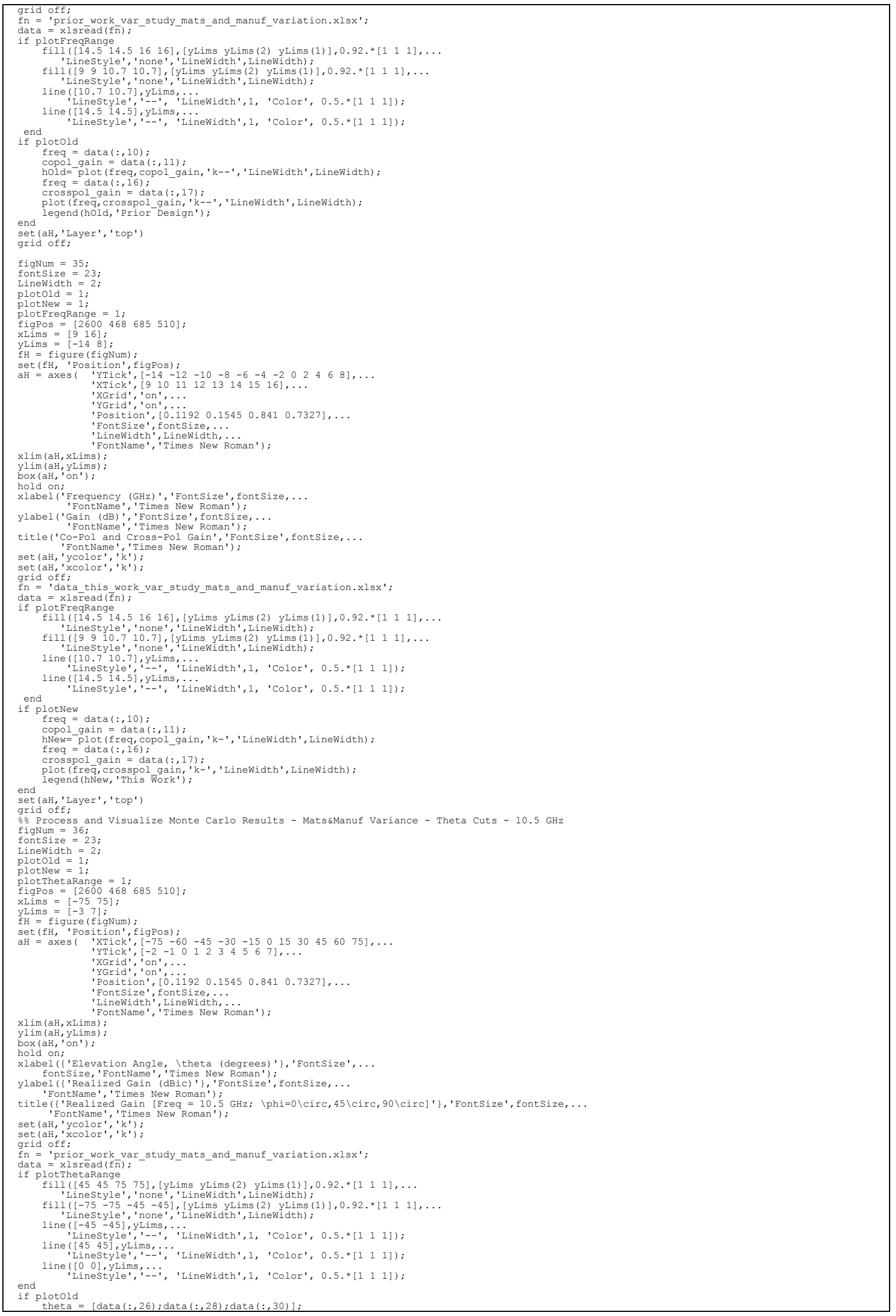




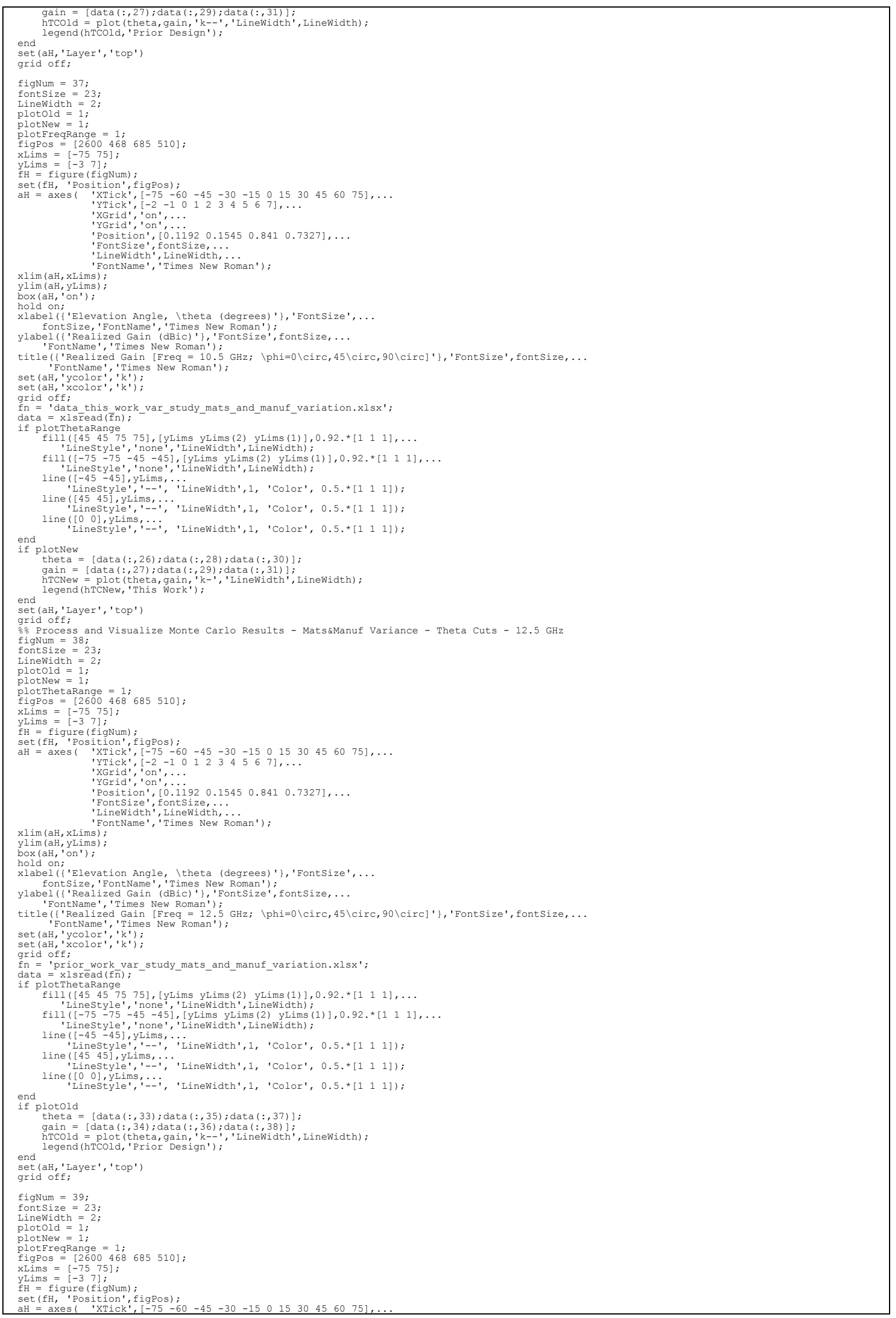




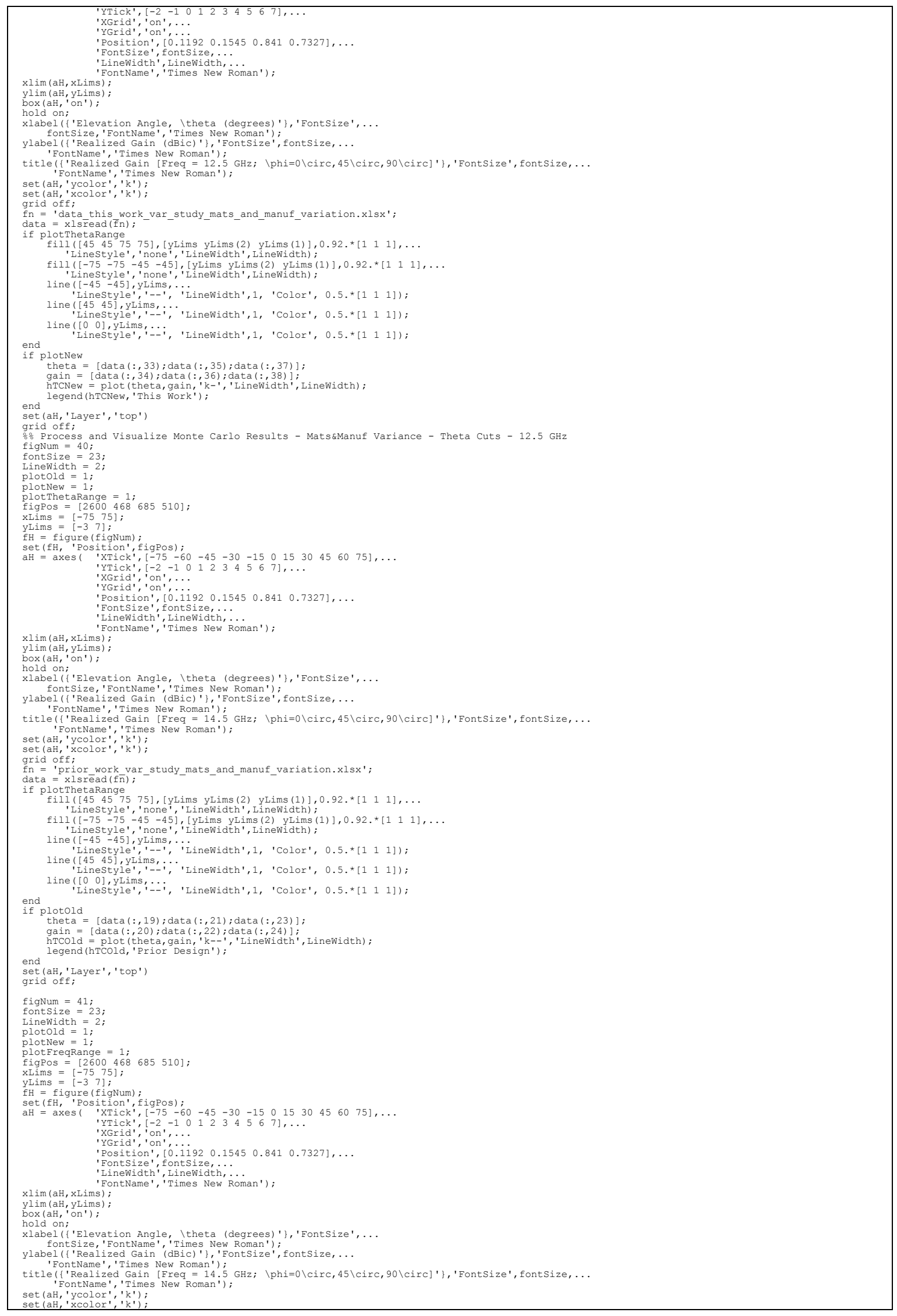




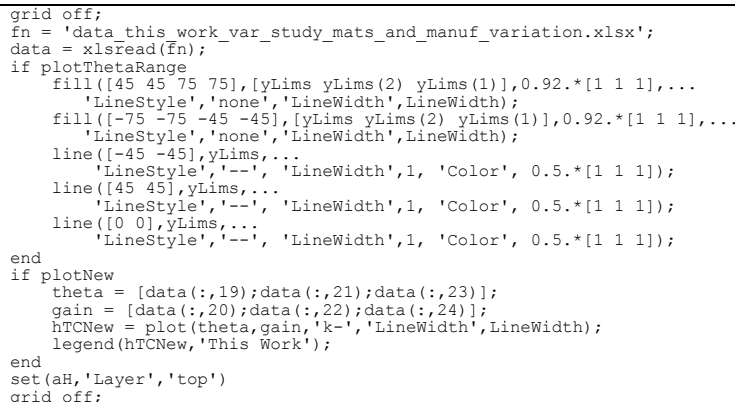

